\title{
MOLECULAR REGULATION OF ENDOTHELIAL NITRIC OXIDE SYNTHASE
}

\author{
By
}

Paul B. Lane, BSc (Hons)

A thesis submitted in accordance with the requirements of the University of Surrey for the Degree of Doctor of Philosophy

Department of Pharmacology, October, 2000

Weill Medical College of Cornell University,

New York, NY 10021

Pharmacology Research Group,

School of Biological Sciences,

University of Surrey,

Guildford, Surrey, GU2 5XH 


\section{IMAGING SERVICES NORTH}

Boston Spa, Wetherby

West Yorkshire, LS23 7BQ

www.bl.uk

\section{PAGE 236,FIG 4.24 \\ PLEASE REFER TO THE \\ ORIGINAL TEXT TO SEE \\ THIS MATERIAL}




\section{ACKNOWLEDGEMENTS}

Firstly, I would like to thank Dr. Gross for giving me the opportunity to come to NYC and for his guidance during my research. Thanks for making it both educational and fun, I'll miss your wacky stories about limousines.

Also thanks to Dr. Susanna Hourani for being my supervisor at Surrey.

To all the members of the Gross Lab, both past and present, thanks for making my time here fun. Special thanks must go to Behrad and Monica for their help putting together this thesis. Also to Dr. Qing Liu for the initial studies of ACE-1, data from which contributes to Figs. $3.9-3.18$.

Additionally, I would like to thank Neil Smith, undergrad and postgrad, I couldn't have done it without our happy times in the company of beer, cheers mate. Thanks also to Glyn and Nicole for your continued friendship, you helped make NYC a time to remember.

Finally to my family, your unconditional support never ceases to amaze me. To Mum and Dad, Sandy, Aileen and Sue, I couldn't have chosen better friends. 


\section{PUBLICATIONS}

The following publications arise directly or indirectly from the work described in this thesis:

Salerno, J.C., Harris, D.E., Irizarry, K., Patel, B., Morales, A.J., Smith, S.M., Martasek, P., Roman, L.J., Masters, B.S., Jones, C.L., Weissman, B.A., Lane, P., Liu, Q. \& Gross, S.S. (1997). An autoinhibitory control element defines calcium-regulated isoforms of nitric oxide synthase. J Biol Chem, 272, 29769-77

Lane, P. \& Gross, S.S. (2000). The autoinhibitory control element and calmodulin conspire to provide physiological modulation of endothelial and neuronal nitric oxide synthase activity. Acta Physiol Scand, 168, 53-56.

Lane, P. \& Gross, S.S. (2000) The C-terminus of endothelial nitric oxide synthase impedes calcium/calmodulin-induced enzyme activation and serves as a second autoinhibitory control element. J Biol Chem (submitted) 


\section{SUMMARY}

The three isoforms of nitric oxide synthase (NOS) are classified based on their mode of regulation by calmodulin (CaM). The "calcium-independent" inducible isoform (iNOS) contains tightly-bound CaM and is active at all levels of intracellular calcium. In contrast, the two calcium-dependent isoforms (neuronal, nNOS and endothelial eNOS) are activated by CaM-binding following stimulus-evoked increases in intracellular calcium. However, both the structural basis for these differences in regulation and the molecular basis for CaM-induced cNOS activation remained unclear. Given that the regulation of calmodulin regulated enzyme systems often involves the displacement of an intrinsic autoinhibitory domain, we attempted to identify regions of eNOS which may fulfill this autoinhibory function. Herein, I describe the identification of two autoinhibitory control elements (ACEs) in eNOS, one within the FMN binding domain (ACE-1) and one located at the C-terminus (ACE-2). Together with CaM, these form a tripartite system for the regulation of eNOS. ACE-1/ACE-2 exerting negative effects to attenuate catalytic activity, which are overcome by the conformational changes induced by CaM-binding. The mechanism of ACE-1/ACE-2-mediated inhibition and the alleviation of this inhibition were investigated. 


\section{CONTENTS}

Acknowledgements $\quad$ ii

$\begin{array}{ll}\text { Publications } & \text { jii }\end{array}$

Summary iv

Contents v v

List of Tables $\quad$ xiii

List of Figures xiv

\section{Chapter 1. General Introduction}

$\begin{array}{lll}1.1 & \text { Historical perspective } & 1\end{array}$

1.2 Nitric oxide chemistry and biological functions 5

1.2.1 Reactivity of NO 6

$\begin{array}{lll}\text { 1.2.2 Biological targets of NO } & 10\end{array}$

1.2.2.1 Nitric oxide synthases 11

$\begin{array}{lll}\text { 1.2.2.2 Guanylate cyclase } & 11\end{array}$

1.2.2.3 Enzymes involved in arachidonate metabolism 14

$\begin{array}{lll}\text { 1.2.2.4 Receptors } & 14\end{array}$

$\begin{array}{lll}\text { 1.2.2.5 Signal amplification systems } & 15\end{array}$

$\begin{array}{lll}\text { 1.2.2.6 Energy metabolism } & 16\end{array}$

$\begin{array}{lll}\text { 1.2.2.7 Transcription factors and DNA synthesis } & 17\end{array}$

$\begin{array}{lll}\text { 1.2.2.8 Iron metabolism } & 18\end{array}$

$\begin{array}{lll}\text { 1.2.2.9 Apoptosis } & 19\end{array}$

1.2.2.10 Direct cytotoxicity and cell defense mechanisms 23

$\begin{array}{lll}\text { 1.2.2.11 Other targets } & 24\end{array}$

$\begin{array}{lll}\text { 1.2.3 Summary } & 25\end{array}$ 
$1.3 \quad$ Nitric oxide synthases

1.4 NOS: gene structure, regulation and expression

1.4.1 iNOS

1.4.2 nNOS

eNOS

NOS structure

$1.6 \quad$ Mechanism and possible roles of cofactors and prosthetic groups

$\begin{array}{lll}1.6 .4 & \text { Tetrahydrobiopterin } & 50\end{array}$

1.6.5 Overall mechanism $\quad 54$

$1.7 \quad$ Regulation of NOS activity $\quad 56$

1.7.1 Transcriptional regulation of iNOS 56

\begin{tabular}{ll|} 
1.7.2 & Regulation of NOS by calmodulin
\end{tabular}

1.7.3 Intracellular localization, protein-protein interactions and 62 posttranslational modifications of NOS isoforms

1.7.3.1 iNOS

1.7.3.1.1 Subcellular localization $\quad 63$

1.7.3.1.2 Protein-protein interactions $\quad 63$

$\begin{array}{lll}\text { 1.7.3.1.3 Posttranslational modifications } & 64\end{array}$

$\begin{array}{lllll}1.7 .3 .2 & \text { nNOS } & & & \end{array}$

$\begin{array}{lll}\text { 1.7.3.2.1 Subcellular localization } & 64\end{array}$

$\begin{array}{lll}\text { 1.7.3.2.2 } \quad \text { Protein-protein interactions } & 65\end{array}$

$\begin{array}{lll}\text { 1.7.3.2.3 Posttranslational modification } & 67\end{array}$ 
1.7.3.3.1 Subcellular localization $\quad 68$

$\begin{array}{lll}\text { 1.7.3.3.2 Protein-protein interactions } & 69\end{array}$

1.7.3.3.3 Posttranslational modification 72

$\begin{array}{lll}\text { 1.7.3.3.3.1 Phosphorylation } & 72\end{array}$

1.7.3.3.3.2 Acylation
75

1.7.3.4 The caveolin-calmodulin cycle for eNOS activation $\quad 77$

Aims $\quad 81$

Chapter 2. Materials and methods

$2.1 \quad$ Materials $\quad 82$

\begin{tabular}{l|l}
2.2 & Mutagenesis of wildtype eNOS
\end{tabular}

$2.3 \quad$ eNOS expression and purification 83

$2.4 \quad$ SDS-PAGE $\quad 85$

$2.5 \quad$ Spectrophotometry of the CO-bound heme chromophore 86

$2.6 \quad$ Assays of enzyme activity $\quad 86$

2.6.1 Griess Assay $\quad 86$

2.6.2 Assay of NOS activity by myoglobin or NADPH oxidation 87

2.6.3 Reduction of cytochrome $\mathrm{c}$ and ferricyanide $\quad 88$

$\begin{array}{lll}2.6 .4 & \text { Calcineurin activity measurement } & 88\end{array}$

$2.7 \quad$ Assays of NOS-ligand binding $\quad 89$

2.7.1 Assay of $\left.\left[{ }^{[25}\right]\right]$-Calmodulin binding to NOS $\quad 89$

2.7.2 $\quad\left[{ }^{125} \mathrm{I}\right]$-Calmodulin association and dissociation rate measurement $\quad 89$

$\begin{array}{lll}\text { 2.7.3 } & \text { Assay of }\left[{ }^{3} \mathrm{H}\right]-\mathrm{N}^{\mathrm{G}} \text { nitro-L-arginine binding } & 90\end{array}$

$\begin{array}{lll}\text { 2.7.4 } & \text { Preparation of calcium solutions } & 90\end{array}$

$\begin{array}{lll}2.8 & \text { PKA-mediated phosphorylation of eNOS } & 90\end{array}$ 
Chapter 3. Identification and characterization of an autoinhibitory control element within the FMN-binding domain of calcium-regulated isoforms of nitric oxide

$$
\text { synthase }
$$

Introduction elements of cNOSs

3.2.2.3 Synthetic polypeptide effects on NOS activity

3.2.2.4 Synthetic polypeptide effects on CaM-binding

3.2.2.5 Synthetic polypeptide effects on the activity of Calcineurin

3.2.2.6 Effect of synthetic polypeptides on arginine binding

3.2.2.7 An integrated model of $\mathrm{cNOS}$ regulation by $\mathrm{Ca}^{2+} / \mathrm{CaM}$ and the putative autoinhibitory control element 
3.2.2.8 Effects of CaM on the exposure of the insert

3.2.3 Function of the conserved regions of the autoinhibitory control element

3.2.3.1 Alignment of the autoinhibitory control elements of NOS isoforms 130

3.2.3.2 Expression and purification

3.2.3.3 Catalytic activities

3.2.3.4 $\mathrm{Ca}^{2+}$-dependence of calmodulin binding and activation 132

3.2.3.5 Does it effect the $\mathrm{EC}_{50}$ for $\mathrm{Ca}^{2+}$ if the CaM-eNOS complexes 139 are preformed

3.2.3.6 Does the RRKRK motif effect the rate of dissociation of 140 CaM-eNOS complexes

3.2.3.7 The RRKRK motif and eNOS-HSP90 association 140

3.2.4 Phosphorylation of the ACE as a method of eNOS regulation 145

3.2.4.1 Putative phosphorylation sites in ACE regions of cNOSs 145

3.2.4.2 Attenuation of the inhibitory potency of eNOS $_{617-639}$ by 146 phosphorylation at consensus PKA sites

3.2.4.3 In vitro phosphorylation of eNOS by PKA

3.2.4.4 Effect of in vitro phosphorylation on eNOS activity

3.2.4.5 Mutation of $\operatorname{Ser}_{635}$ to mimic phosphorylation isoforms of nitric oxide synthase 
element of eNOS causes enhanced activity of the reductase

domain and an increased rate of NO synthesis

3.3.3 Regulation of eNOS by phosphorylation of a conserved cAMP

dependent protein kinase site within the autoinhibitory control

element

Chapter 4. The C-terminus of eNOS impedes calcium/calmodulin-induced enzyme

activation and serves as a second autoinhibitory control element

$\begin{array}{lll}\text { 4.1 Introduction } & 178\end{array}$

4.2.1 Identification and characterization of an autoinhibitory control 181 element within the C-terminal extension of eNOS

4.2.2 4.2.1.1 Expression and purification

185

4.2.1.2 Catalytic activities of $\mathrm{C} \Delta 27$ and wildtype eNOS 186

4.2.1.3 Effect of a C-terminal extension-derived peptide on eNOS activity 187

4.2.1.4 $\mathrm{Ca}^{2+}$-dependence of calmodulin binding and activation of eNOS 197

4.2.1.5 Calmodulin binding affinity 198

4.2.1.6 Does it effect the $\mathrm{EC}_{50}$ for $\mathrm{Ca}^{2+}$ if the CaM-eNOS complexes 200 are preformed

4.2.2 CaM binding and eNOS activation are distinct and temporally 206 separated events

4.2.2.1 Activation of wildtype and C $\Delta 27$ eNOS by engineered Calmodulin 208 
$\begin{array}{lll}\text { 4.2.3 Regulatory interactions of ACE-2 } & 217\end{array}$

4.2.3.1 Interactions between ACE-1 and ACE-2 217

4.2.3.2 Interaction between ACE-2 and heat shock protein $90 \quad 217$

$\begin{array}{lll}4.3 & \text { Discussion } & 221\end{array}$

4.3.1 How does ACE-2 exert its autoinhibitory effects 223

4.3.2 ACE-2 and "capping" of reductase activity 228

4.3.3 ACE-2 and reductase $\rightarrow$ oxygenase electron transfer 229

4.3.4 Binding of CaM and activation of eNOS are related, yet distinct 230 processes

4.3.5 A physiological model of eNOS activation

4.3.6 ACE-2 and $\mathrm{Ca}^{2+} / \mathrm{CaM}$-dependence of eNOS activation 235

$\begin{array}{lll}\text { 4.3.7 Insights into eNOS regulation by AKT/PKA-mediated } & 239\end{array}$ phosphorylation of $\operatorname{Ser}_{1179}$.

\section{Chapter 5. Summary}

5.1 ACE-1, ACE-2 and the CaM-binding site:

a tripartate system for regulation of $\mathrm{cNOS}$

\section{Chapter 6. Future work}

6.4 HSP90 and $\mathrm{Ca}^{2} / \mathrm{CaM}$ binding 
7.

References 


\section{LIST OF TABLES}

$\begin{array}{lll}1.1 \quad \text { Biological targets of NO signaling } & 12\end{array}$

$1.2 \quad$ Purified nitric oxide synthases 28

$\begin{array}{lll}\text { 1.3 Cloned nitric oxide synthase isoforms } & 30\end{array}$

2.1 Sequences of primer pairs for individual eNOS mutations 84

3.1 FMN insert-derived peptide sequence and origin $\quad 113$

3.2 Summary of the effects of FMN insert-derived peptides on NOS activity 114 and ligand binding

\section{LIST OF FIGURES}

1.1 Overview and comparison of the modular structure of NOS isoforms 31

1.2 Formation of the NOS dimer $\quad 41$

1.3 Summary of the NOS catalyzed reaction 51

1.4 Schematic representation of electron flux through NOS 52

1.5 Schematic representation of iNOS induction $\quad 57$

1.6 The caveolin-calmodulin cycle for eNOS activation $\quad 80$

3.1 SDS-polyacrylamide gel electrophoresis of purified recombinant NOSs. 98

3.2 Stability of NOS homodimer by low-temperature SDS-polyacrylamide 99 gel electrophoresis.

3.3 P450 spectra of eNOS, nNOS and iNOS 100

3.4 Rates of NO synthesis of the three NOS isoforms 101

3.5 $\mathrm{Ca}^{2+}$-dependence of the three isoforms of NOS 102

3.6 Sequence alignment of NOS isoforms and related proteins showing $\quad 108$ location and conservation of the FMN insertion 
3.7 Structural models of the FMN binding domains of NOS isoforms and related flavoproteins.

3.8 Structural models of the FMN binding domains of eNOS and iNOS with 110 bound-CaM.

3.9 Inhibitory effect of NOS $_{617-639}$ on the activity of the three NOS isoforms. 112

3.10 Effect of eNOS $601-633$ and NNOS $_{607-634}$ on [ $\left.{ }^{125} \mathrm{I}\right]-\mathrm{CaM}$ binding to eNOS and 118 nNOS.

3.11 Effect of $\mathrm{Ca}^{2+}$ concentration on the peptide-dependent inhibition of $\left[{ }^{125} \mathrm{I}\right]-\mathrm{CaM}$ binding to nNOS.

3.12 Peptide-dependent inhibition of nNOS activity is fully reversible by the addition of excess calmodulin.

3.13 Competitive inhibition of [ $\left.{ }^{125} \mathrm{I}\right]-\mathrm{CaM}$ binding to $\mathrm{nNOS}$ by insertion-derived 121 peptide $\operatorname{eNOS}_{607-634}$.

3.14 Direct binding of $\left[{ }^{125} \mathrm{I}\right]-\mathrm{CaM}$ to NNOS and $\mathrm{eNOS}_{607-634}$

3.15 Acceleration of $\left[{ }^{125} \mathrm{I}\right]-\mathrm{CaM}$ dissociation from cNOSs by eNOS $_{607-634}$.

3.16 Relative effects of the insert-derived peptides on the activity of cNOss and calcineurin.

3.17 SDS-PAGE showing the products of limited tryptic digestion of nNOS

3.18 SDS-PAGE showing the products of limited tryptic digestion of nNOS

3.19 Regions of conservation within the FMN-insertions of eNOS and nNOS. 133

3.20 SDS-PAGE of purified recombinant RRKRK del and RRKRK sub eNOS. 134

3.21 Stability of RRKRK mutant eNOS homodimers by low-temperature SDS-PAGE.

3.22 P450 Spectra of wildtype, RRKRK del and RRKRK sub eNOS

3.23 Rates of NO synthesis of wildtype, RRKRK del and RRKRK sub eNOS 
3.24 Reductase activities of wildtype, RRKRK del and RRKRK sub eNOS 138

3.25 Calcium-dependence of NO synthesis and calmodulin-binding for wildtype and RRKRK mutant eNOSs.

3.26 Calcium-dependence of activation vs. deactivation for wildtype and RRKRK del eNOS.

3.27 Rate of $\left[{ }^{125} \mathrm{I}\right]-\mathrm{CaM}$ dissociation from wildtype and RRKRK del eNOS. 143

3.28 Effect of HSP90 on the activity of wildtype and RRKRK del eNOS. 144

3.29 Conservation of PKA/PKG concensus sites in eNOS 151

3.30 Attenuation of the inhibitory potency of $\mathrm{eNOS}_{617-639}$ by phosphorylation 152 at consensus cAMP-dependent protein kinase sites.

$3.31{ }^{32} \mathrm{P}-\mathrm{Phosphate}$ incorporation into wildtype eNOS by PKA.

3.32 ${ }^{32} \mathrm{P}$-Phosphate incorporation into wildtype and $\mathrm{Ala}_{635 / 636}$ eNOS by PKA. $\quad 154$

3.33 ${ }^{32} \mathrm{P}$-Phosphate incorporation into wildtype and $\mathrm{Ala}_{494 / 635 / 636}$ eNOS by PKA 155

3.34 Confirmation of $\operatorname{Ser}_{635 / 636}$ as a site of PKA-mediated phosphorylation by 156 electrospray ionization using selected ion recording.

3.35 Effect of PKA-mediated phosphorylation on the observed calcium-

dependence of wildtype eNOS.

3.36 Effect of PKA-mediated phosphorylation on the rate of NO synthesis by eNOS

3.37 Effect of PKA-mediated phosphorylation on the rates of ferricyanide and cytochrome c reduction.

3.38 Rates of NO synthesis of wildtype, $\mathrm{Glu}_{635}$ and $\mathrm{Ala}_{635}$ eNOS.

3.39 Rate of $\left[{ }^{125} \mathrm{I}\right]-\mathrm{CaM}$ dissociation from wildtype and $\mathrm{Glu}_{635}$ eNOS.

4.1 Sequence alignment of various NOS isoforms and cytochrome P450 
reductase.

$\begin{array}{lll}4.2 & \text { Structure of cytochrome P450 reductase. } & 184\end{array}$

4.3 SDS-polyacrylamide gel electrophoresis of purified C $\Delta 27$ eNOS. $\quad 189$

4.4 P450 spectra of wild type and C $\Delta 27$ eNOS. $\quad 190$

4.5 Stability of NOS homodimer by low-temperature SDS-polyacrylamide 191 gel electrophoresis.

4.6 Rates of NO synthesis by wildtype and C $\Delta 27$ eNOS. $\quad 192$

$\begin{array}{lll}4.7 & \text { Reductase activities of wildtype and C } \Delta 27 \text { eNOS. }\end{array}$

4.8 Effect of eNOS ${ }_{1179-1205}$ on the rate of NO synthesis of wildtype and C $\Delta 27 \quad 194$ eNOS

4.9 Effect of eNOS ${ }_{1179-1205}$ on the rate of ferricyanide reduction by wildtype 195 and $\mathrm{C} \Delta 27$ eNOS.

4.10 Effect of eNOS ${ }_{1179-1205}$ on the rate of cytochrome c reduction by wildtype 196 and $\mathrm{C} \Delta 27$ eNOS.

4.11 Calcium-dependence of NO synthesis and calmodulin-binding for 201 wildtype and $C \triangle 27$ eNOS.

4.12 Calmodulin affinity for wildtype and C $\Delta 27$ eNOS. 202

4.13 Rate of $\left[{ }^{125} \mathrm{I}\right]-\mathrm{CaM}$ dissociation from wildtype and and $\mathrm{C} \Delta 27$ eNOS. 203

4.14 rate of $\left[{ }^{125} \mathrm{I}\right]-\mathrm{CaM}$ association to wildtype and and $\mathrm{C} \Delta 27$ eNOS.. 204

4.15 Calcium-dependence of activation vs. deactivation for wildtype 205 and $\mathrm{C} \triangle 27$ eNOS..

4.16 Comparison of calcium-dependencies for CaM-binding and activation of 211 wildtype and $\mathrm{C} \Delta 27$ eNOS..

4.17 Inhibition of [ $\left.{ }^{125} \mathrm{I}\right]-\mathrm{CaM}$-binding to wildtype and C $\Delta 27$ eNOS by 212 engineered CaM proteins 
4.18 Rates of NO synthesisby wildtype and C $\Delta 27$ eNOS in the presence of engineered CaM proteins.

4.19 Ability of engineered CaM proteins to elicit activation of cytochrome c reduction by wildtype and $\mathrm{C} \triangle 27$ eNOS..

4.20 Ability of engineered CaM proteins to elicit activation of ferricyanide reduction by wildtype and $\mathrm{C} \Delta 27 \mathrm{eNOS}$.

4.21 Calcium-dependence for activation by NN-CaM of NO synthesis by $\quad 216$ wildtype and $\mathrm{C} \triangle 27$ eNOS.

4.22 Inhibition of wildtype and C $\Delta 27$ eNOS by eNOS $_{617-639}$, a peptide derived 219 from the FMN autoinhibitory control element (ACE-1).

4.23 Effect of HSP90 on the activity of wildtype and C $\triangle 27$ eNOS.

4.24 Structure of CaM.

4.25 Four-step model of eNOS activation by $\mathrm{Ca}^{2+} / \mathrm{CaM}$.

4.26 Conservation of the AKT phosphorylation site throughout the cNOSs. 241 


\section{Chapter 1}

\section{General Introduction}

\subsection{Historical Perspective}

Although it was known since the late 1970's that nitric oxide (NO) is among the ligands that can activate soluble guanylate cyclase and cause vascular relaxation (Arnold et al., 1977; Craven \& De Rubertis, 1978; Gruetter et al., 1979), the molecule was generally regarded as an environmental pollutant. It was not until a decade later that NO was recognized as an endogenous vasodilator.

The concept of NO as a biological messenger was first proposed by investigators studying a labile substance released from the vascular endothelium in response to acetylcholine and other agonists. In a landmark study it was demonstrated that the relaxation of blood vessels by acetylcholine required an intact endothelium (Furchgott \& Zawadzki, 1980). They proposed that the stimulation of muscarinic receptors on the endothelium stimulated the release of a labile substance from the endothelium causing the relaxation of the vascular smooth muscle. This labile substance was termed endothelium-derived relaxing factor (EDRF) and was found to increase cGMP in vascular smooth muscle (Holzmann, 1982), activate soluble guanylate cyclase (Forstermann et al., 1986) and inhibit platelet aggregation (Azuma et al., 1986).

In 1986, at the Fourth Symposium on Mechanisms of Vasodilation, R.F. Furchgott and L.J. Ignarro noted the similarities between NO and EDRF, in that they both relax vascular smooth muscle and are similarly quenched by hemoglobin and 
superoxide. They proposed that EDRF is either NO or a labile compound capable of releasing NO (Furchgott, 1988; Ignarro, 1988). It was later demonstrated experimentally that the release of NO or an NO precursor accounted for the biological activities of EDRF (Ignarro et al., 1987; Palmer et al., 1987). This identification was based on the accumulation of nitrite in bioassays, and the similarities in pharmacological and biochemical properties of NO and EDRF (Ignarro, 1989).

The general acceptance of this hypothesis was hindered by the lack of an identifiable enzymatic pathway for the synthesis of NO in mammals. It had been suggested that humans could synthesize nitrogen oxides from early nutritional studies by Mitchell in 1916 (Mitchell et al., 1916), but it was generally believed that mammalian excretion of nitrate and nitrite was due to denitrification reactions by enteric bacteria. Interest in the origin of excreted nitrite intensified, based on concerns that nitrite may serve as a precursor to the carcinogenic $\mathrm{N}$-nitroso compounds (Bogovski \& Bogovski, 1981) and therefore involved in the etiology of cancers. Further evidence that humans could endogenously produce nitrogen oxides was obtained from studies of nitrate metabolism; fed a low protein diet, humans were found to produce more nitrate and nitrite than they ingested (Green et al., 1981; Tannenbaum et al., 1978). The involvement of enteric microflora in nitrate production was precluded by studies using germ-free rats (Green et al., 1981). It was additionally noted that one human subject developed a fever during which excreted levels of nitrate were significantly increased. Subsequent studies showed that urinary nitrate excretion was increased in rats following challenge with bacterial endotoxin (Wagner et al., 1983) and suggesting the involvement of the reticuloendothelial system. Later, in vitro studies identified macrophages as a source of urinary nitrate in 
bacterial lipopolysaccharide (LPS) treated mice and of endogenously synthesized nitrogen oxides (Stuehr \& Marletta, 1985).

Cytotoxic activated macrophages caused the metabolic inhibition of tumor target cells involving the inhibition of mitochondrial respiration, aconitase, DNA synthesis and the loss of intracellular iron. This pattern of metabolic inhibition was found to stereospecifically require L-arginine and was paralleled by increases in nitrate and nitrite (Hibbs et al., 1987). Isotope studies using ${ }^{15} \mathrm{~N}$ - and ${ }^{14} \mathrm{C}$-labelling showed that the nitrogen atom of the nitrogen oxides was derived from one of the guanido nitrogens of L-arginine and revealed citrulline to be a co-product (Iyengar et al., 1987). Further evidence was provided by the fact that secondary amines, such as morpholine can be nitrosylated by immunostimulated macrophages and that this process was dependent on L-arginine (Miwa et al., 1987). Since this nitrosylation reaction could not be carried out by either nitrate or nitrite, it was concluded that a more reactive nitrogen species must be formed from $\mathrm{L}$-arginine as a reaction intermediate in the synthesis of nitrate and nitrite.

The discovery of this novel macrophage pathway indicated a possible method for the synthesis of nitric oxide in endothelium. In 1988 several groups demonstrated that L-arginine was the precursor of EDRF/NO (Palmer et al., 1988; Sakuma et al., 1988). The pathway in endothelial cells was also found to be dependent on L-arginine and inhibited by $\mathrm{N}$-guanido substituted arginine based compounds, such as $\mathrm{N}^{\mathrm{G}}$ methyl-L-arginine (L-NMA) (Palmer et al., 1988; Schmidt et al., 1988; Schmidt et al., 1988). At around the same time, the cytotoxic effector produced by activated 
macrophages was identified as nitric oxide (Hibbs et al., 1988; Marletta et al., 1988; Stuehr et al., 1989; Stuehr \& Nathan, 1989).

The idea of a widespread distribution and a variety of functions for this novel pathway came from research carried out in another seemingly unrelated area of study, the central nervous system (CNS). It was observed that cGMP levels were elevated following activation of NMDA receptors. The increase in cGMP levels was found not in the cells which received the initial stimulus, but in those nearby. This implied the existence of an intercellular signaling system . Earlier studies on rat forebrain identified arginine as the endogenous activator of guanylate cyclase (Deguchi \& Yoshioka, 1982). Further investigation led to the observation that cerebellar cells released an activator of guanylate cyclase with similar stability to EDRF/NO (Garthwaite et al., 1988). The synthesis of nitric oxide has since been demonstrated in a variety of cell types in addition to those mentioned earlier. These include cytokine activated hepatocytes (Billiar et al., 1990; Curran et al., 1989; Nussler et al., 1992), neutrophils (Rimele et al., 1988; Wright et al., 1989), hepatic Kupffer cells (Billiar et al., 1989), vascular smooth muscle (Marsden \& Ballermann, 1990), chondrocytes (Stadler et al., 1991), endothelial cells (Gross et al., 1991; Kilbourn \& Belloni, 1990), adrenal glands (Palacios et al., 1989), and platelets (Radomski et al., 1990).

Hence in a little over a decade, research in the fields of physiology, immunology and neuroscience converged to study a common pathway and nitric oxide emerged as a unique biological messenger molecule regulating cellular function. The importance of this field was recognized recently by the award of the 
1998 Nobel Prize for medicine to Drs. Ignarro, Furchgott and Murad, pioneers of NO biology.

\subsection{Nitric Oxide Chemistry and Biological Functions}

NO is a lipophilic, highly reactive, free radical gas with diverse biomessenger functions. Evidence reveals an involvement of NO in a remarkable array of key physiological processes, including regulation of vascular tone (Furchgott, 1988; Ignarro, 1988; Ignarro et al., 1987; Palmer et al., 1987), platelet aggregation, (Azuma et al., 1986) host-defense (Hibbs et al., 1987; Hibbs et al., 1987; Iyengar et al., 1987; Marletta et al., 1988), inflammation (Harbrecht et al., 1995; Tiao et al., 1994), neurotransmission (Bredt et al., 1990), learning and memory (Bohme et al., 1993; Chen et al., 1997; Holscher \& Rose, 1992; Kendrick et al., 1997; Prickaerts et al., 1997; Rickard et al., 1998; Yamada, 1998), penile erection (Argiolas, 1994; Burnett et al., 1992; Hellstrom et al., 1994; Jung et al., 1997; Melis \& Argiolas, 1995; TrigoRocha et al., 1993), gastric emptying (Konturek et al., 1995; Orihata \& Sarna, 1994; Plourde et al., 1994; Takakura et al., 1997), hormone release (McCann et al., 1997; Rettori et al., 1993; Seilicovich et al., 1995), cell differentiation (Morbidelli et al., 1996; Peunova \& Enikolopov, 1995), cell migration (MacIntyre et al., 1991; Noiri et al., 1996) and apoptosis (Albina et al., 1993; Beauvais et al., 1995; Chun et al., 1995; Mannick et al., 1994; Messmer et al., 1996; Sarih et al., 1993). These biological actions of NO result from covalent modification of numerous enzymes, receptors, structural proteins and transcription factors (see Table 1.1). Whether the net effects of NO on a given cell are either beneficial or deleterious will be determined by the NO 
concentration obtained, duration of production and composition of the surrounding microenvironment.

Appreciation of the role of nitric oxide (NO) in mammalian cell biology has toppled the paradigm that biological signaling is initiated exclusively by noncovalent, lock-and-key type interactions with receptor proteins. Remarkably, nitric oxide is a free-radical that signals by chemical reaction with its protein targets, resulting in covalent modifications and often a stable alteration in protein structure and function. While most proteins may be coerced to react with NO in vitro, the specific proteins that are functionally modified by NO within cells will depend upon the concentration of NO and the composition of the intracellular milieu. A further level of complexity is introduced into NO signaling by the fact that reactions can occur with NO directly, or secondarily with NO-derived species. Much to the surprise of those who thought that reactive molecules are generated and act only under pathophysiological conditions (e.g., ischaemia-reperfusion injury), NO has emerged as a prototype molecule that signals by chemistry in normal physiology.

\subsubsection{Reactivity of NO}

In pure aqueous solution, nanomolar concentrations of NO have been reported to survive with a half-life of 500 seconds or longer (Wink et al., 1993). In contrast, a measured half-life of only 5 seconds has been reported for endogenously produced NO in a physiological milieu (Lancaster, 1994). The rapid physiological inactivation of NO is attributed to reaction with components of physiological fluids and buffers. 
Therefore, critical to our understanding of the signaling capabilities of NO, a relatively stable free radical gas, is an appreciation of the range of possible and dominant reactions of NO in biological systems. The most preferred chemical targets of NO include reactive oxygen species, other free radicals, transition metals, thiols and molecular oxygen. Along with NO itself, the products of these reactions, peroxynitrite (ONOO), metal-nitrosyl adducts (M-NO), S-nitroso products, higher nitrogen oxides (NOx) and other derived species, contribute to the biological actions of arginine-derived NO. Thus, the consequences of NO synthesis, beneficial or deleterious, will dependent on the species and concentration of intermediates formed and the nature and complexity of available targets for reaction (Stamler, 1994). To understand the biological chemistry of NO, it may be useful to consider NO and derived species as existing in a redox array $\left(\mathrm{NO}, \mathrm{NO} \bullet\right.$ and $\left.\mathrm{NO}^{+}\right)$of interconvertable species with distinct chemical characters and reactivities (for review see Stamler $e t$ al., 1992).

In addition to being mediators of the biological effects of NO, it has been suggested that S-nitrosothiols can act as NO reservoirs or NO substitutes (Gaston $\boldsymbol{e t}$ al., 1994; Stamler et al., 1992). It should be noted however that NO does not react directly with thiols to form S-nitrosothiols; redox activation of either the thiol to thiyl, or NO to a species capable of nitrosation $\left(\mathrm{NO}^{+}, \mathrm{N}_{2} \mathrm{O}_{3}\right)$ appears to be necessary. $\mathrm{NO}$ reacts with $\mathrm{O}_{2}{ }^{-}$and transition metals to form ONOO- (Beckman et al., 1990) and a species with nitrosonium character $\left(\mathrm{NO}_{2}{ }^{+}\right)$(Ischiropoulos et al., 1992). These species are each capable of supporting additional nitrosative reactions at nucleophillic centers in tyrosine residues and thiol groups, resulting in the formation of nitrotyrosine and Snitrosothiols. A nitrosating species can also arise from the reaction between NO and 
ferro-heme in hemoproteins, such as hemoglobin and myoglobin. In this reaction ferric iron is reduced to the ferrous form, resulting in the formation of an adduct with nitrosonium character $\left(\mathrm{NO}^{+}\right)$that can react to produce S-nitrosothiols. It should be appreciated that reaction of NO with ferri-heme is much slower than that with ferroheme; the latter reaction occurs at a near diffusion-limited rate. Only at relatively high levels of biologically-derived NO may molecular oxygen become a significant target, resulting in the production of the very potent nitrosating species, $\mathrm{NO}_{2}{ }^{+}$.

As noted above, a preferred reaction of NO is with superoxide anion $\left(\mathrm{O}_{2}{ }^{\circ}\right)$, to form peroxynitrite ( $\left.\mathrm{ONOO}^{-}\right)$. This reaction may be most important under biological conditions where abundant NO and superoxide are simultaneously produced, such as during inflammation, endotoxemia and ischaemia/reperfusion. The resulting peroxynitrite is a much stronger oxidant than either the NO or superoxide, and appears to be a key bacteriocidal product of immunostimulant-activated macrophages (Zhu et al., 1992). Under physiological conditions peroxynitrite is sufficiently stable to diffuse some distance before reacting with target molecules such as membrane lipids (Radi et al., 1991), protein sulfhydryl groups (Radi et al., 1991), DNA (Inoue \& Kawanishi, 1995; Wink et al., 1991), and antioxidants (Hogg et al., 1994). Peroxynitrite, when produced by endothelial cells, is thought to contribute to endothelial injury via modification of the low density lipoprotein within the arterial wall (White et al., 1994), a process which may contribute to formation of the fatty streak and plaque that are characteristic of the atherosclerotic lesion (Beckman et al., 1990; White et al., 1994). Peroxynitrite also has been implicated in the nitration of tyrosine residues in histone, lysozyme, and superoxide dismutase (Ischiropoulos et 
al., 1992). However, more recent evidence suggests that another species, possibly - $\mathrm{NO}_{2}$, is most responsible for tyrosine nitration in vivo (Pfeiffer \& Mayer, 1998).

Regardless of the mediator responsible, tyrosine nitration has been identified in several pathological conditions. There are several possible ways in which tyrosine nitration could influence cellular signaling. While nitration may inhibit tyrosine phosphorylation and associated signal transduction (Martin et al., 1990), it may also mimic the effects of tyrosine phosphorylation (Berlett et al., 1996). Nitration may also alter protein function/activity by inducing distinct conformational changes (DeckersHebestreit et al., 1987; Lundblad et al., 1988) or cause proteins to be targeted for proteolysis. Nitrated tyrosines structurally resemble dinitrophenol, a strongly antigenic compound, and have therefore been implicated in the initiation of autoimmune responses (Cross et al., 1997; van der Veen et al., 1997). Nitrated tyrosines have also been implicated in systems essential to the survival of motor neurons (Nixon, 1993).

In addition to causing tyrosine nitration, peroxynitrite can also interact directly with iron-sulfur clusters and zinc-thiolate centers contained in many enzymes and transcription factors (Drapier et al., 1993; Hibbs et al., 1990; Kroncke et al., 1994; Pantopoulos \& Hentze, 1995; Stuehr \& Nathan, 1989; Weiss et al., 1993; Zeng et al., 1991). By this route, peroxynitrite may have effects on metabolic pathways, DNA repair, gene expression and various other cellular effects attributed to NO.

Nitric oxide also undergoes rapid reaction with other biological free radicals. In conditions such as inflammation or oxidative stress, hydroxyl radical $(\mathrm{OH} \bullet)$ and 
lipid species such as peroxyl (LOO•) and alkoxyl (LO•) radicals, are generated by the fenton reaction and lipid peroxidation. As NO• reacts with these radicals at near diffusion-limited rates, it can act as a potent scavenger of OH• (Rubbo et al., 1994) and hence inhibit the radical-induced chain propagation reaction in lipid peroxidation (Padmaja \& Huie, 1993). Also, because of its high lipophilicity, NO concentrates in membranes and can act as a vitamin E-like antioxidant in oxidant-mediated lipid peroxidation. During bacterial infection, phagocytes are rapidly activated to produce cytotoxic reactive oxygen species (ROS) and subsequently become activated to produce NO. In addition to the desirable bacteriocidal effects of ROS, these species can damage host cells by initiating lipid peroxidation, as noted above. The delayed production of NO may serve to terminate lipid radical-induced chain propagation and hence limit host cell damage. NO can also react with highly oxidized iron, $\mathrm{Fe}(\mathrm{IV})=\mathrm{O}$, a potent oxidizing species produced by reaction with hemoproteins, and inhibit lipid peroxidation of LDL.

\subsubsection{Biological Targets of NO}

The biological activities of NO can be attenuated by competing targets, such as superoxide anion, ferro-hemoproteins, and thiol-containing compounds. Thus, actual reactions of NO obtained with potential biological targets will be dependent on the local NO concentration as well as the specific components of the surrounding microenvironment (e.g. availability of alternate targets and intracellular redox potential). It should be considered that the concentration of NO required to elicit a given biological response will vary from target to target, with some reactions occurring at low physiologically-occurring concentrations of NO (e.g. activation of 
guanylate cyclase) and others requiring pathophysiological concentrations of NO (e.g. inhibition of cytochrome P450). Key reactions of NO with potentially important biological targets are outlined below and summarized in Table 1.1.

\subsubsection{Nitric Oxide Synthases}

Due to the role of ferro-heme in NOS catalysis, and the high affinity of NO for heme, NOS is a most obvious target for NO. Indeed it has been shown that NO can inhibit its own biosynthesis by heme binding in NOS (Abu-Soud et al., 1995; Buga et al., 1993; Griscavage et al., 1993). This feedback inhibition may serve to regulate neurotransmitter release, blood vessel relaxation and NO-mediated cytotoxicity.

\subsubsection{Guanylate Cyclase}

Guanylate cyclase is an important hemo-protein target for NO, binding of NO alters the conformation of the heme moiety and causes activation of the enzyme. The subsequent rise in cGMP levels causes the activation of cGMP-dependent kinases, which mediate smooth muscle relaxation, platelet adhesion and aggregation, and contribute to neuron signaling. cGMP elevation may also cause the phosphorylation of synaptic vesicle proteins associated with the release of neurotransmitters (Montague et al., 1994), open

cGMP-gated ion channels to increase intracellular $\mathrm{Ca}^{2+}$ levels, and modulate the activity of phosphodiesterase isoforms to control intracellular cAMP levels (Schmidt et al., 1993). 
Table 1.1 Biological Targets of NO Signaling

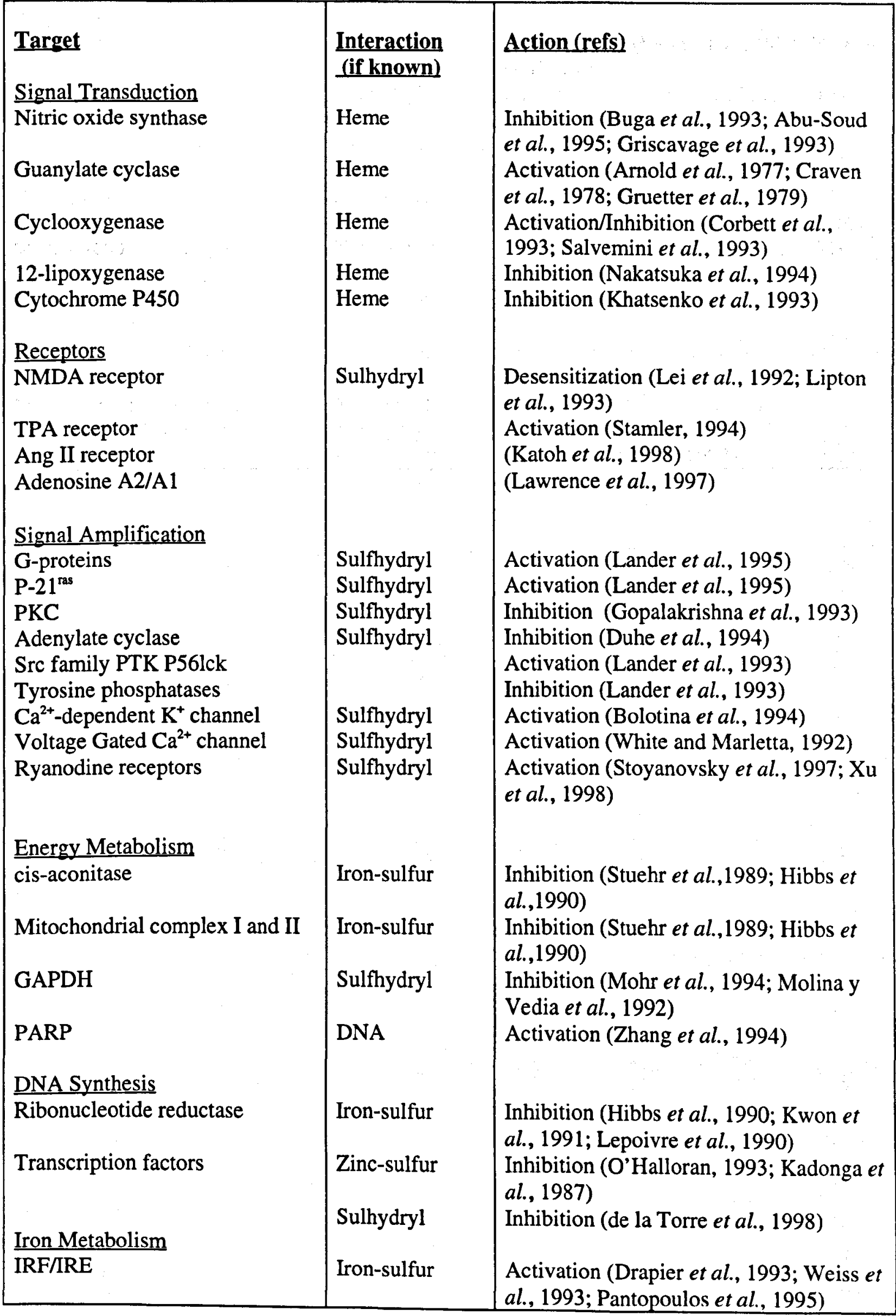




\begin{tabular}{|c|c|c|}
\hline Apoptosis & & \\
\hline DNA & Amines & $\begin{array}{l}\text { Strand breaks (Inoue et al., 1995, Nguyen } \\
\text { et al., 1992) }\end{array}$ \\
\hline Caspases & Sulhydryl & $\begin{array}{l}\text { Inhibition (Dimmeler et al., 1997; Li et } \\
\text { al., 1997) }\end{array}$ \\
\hline Direct Cytotoxicity & & $x^{2}$ \\
\hline Glutathione & Sulfhydryl & $\begin{array}{l}\text { Redox signaling (Walker et al., 1995; } \\
\text { Nikitovic et al., 1996; Hou et al., 1996) }\end{array}$ \\
\hline $\mathrm{Mn} / \mathrm{Fe} \mathrm{SOD}$ & Tyrosine & Inhibition (Ischiropoulos et al., 1992) \\
\hline Glutathione reductase & Sulhydryl & Inhibition (Wharton et al., 1988) \\
\hline Catalase & Heme & Inhibition (Brown et al., 1995) \\
\hline Other & & $\therefore \ldots$ \\
\hline Methionine adenosyltransferase & Sulfhydryl & Inhibition (Ruiz et al., 1998) \\
\hline Alcohol dehydrogenase & Sulfhydryl & Inhibition (Gegel et al., 1996) \\
\hline Caeruloplasmin & Copper & Inhibition (Swain v 1994) \\
\hline
\end{tabular}




\section{1,2.2.3 Enzymes Involved in Arachidonate Metabolism}

The metabolism of arachidonic acid results in the production of various biologically active lipid mediators. Cyclooxygenase is the rate-limiting enzyme in the synthesis of arachidonate-derived prostaglandins, thromboxane $A_{2}$ and prostacyclins. There is evidence for both activation (Corbett et al., 1993; Salvemini et al., 1993) and inhibition (Kanner et al., 1992) of this process by NO interaction with the heme group of both cyclooxygenase types I and II. Other hemo-proteins involved in arachidonate metabolism, including the 12-lipoxygenase in platelets (Nakatsuka \& Osawa, 1994) and cytochromes P450 (Khatsenko et al., 1993; Stadler et al., 1994) are inhibited by NO and nitrovasodilator drugs. Hence it would seem that NO has the capability to modulate the production of prostaglandins and other bioactive metabolites of arachidonate, as well as the metabolism of numerous endogenous and exogenous compounds by cytochromes $\mathrm{P} 450$.

\subsubsection{Receptors}

Stimulation of N-methyl-D-aspartate (NMDA) receptors results in the production of NO, and overstimulation may result in neurotoxicity due to reactions of NO with $\mathrm{O}_{2}^{-}$ to form ONOO- (Lei et al., 1992; Lipton et al., 1993). However, nitrosation of a critical thiol on the NMDA receptor by an NO derived species, leads to receptor desensitization and neuroprotection. This desensitization seems to result from the subsequent formation of an intramolecular protein disulphide bond (Lei et al., 1992; Lipton et al., 1993). Hence, it seems that although contributing to the toxicity caused by overstimulation by NMDA, NO can also protect against this amino acid excitotoxicity by desensitization of NMDA receptors. The activation of tissue specific 
plasminogen activator receptors also seems to occur by the post-translational modification of a single thiol (Stamler et al., 1992).

More recently, chronic inhibition of NO production has been demonstrated to cause an upregulation of angiotensin II (AngII) receptors in a rat model (Katoh et al., 1998) and NO has been suggested to interact with the adenosine A2A and A1 receptors in rat vagal afferent neurons (Lawrence et al., 1997). However, the physiological relevance and effects of these interactions remain to be assessed.

\subsubsection{Signal Amplification Systems}

NO has been reported to activate pertussis toxin-sensitive G-proteins and p21ras (Lander et al., 1995) by S-nitrosylation. This same modification causes the inhibition of activity for both protein kinase $\mathrm{C}$ and type I adenylate cyclase (Duhe $e t$ al., 1994; Gopalakrishna et al., 1993). Studies using NO donor compounds in human peripheral blood mononuclear cells indicate that $\mathrm{NO}$ activates the src family protein tyrosine kinase p561ck and inhibits tyrosine phosphatase activity (Lander et al., 1993). Also NO has been shown to activate the calcium-dependent potassium channel in smooth muscle, activation of this channel occurs by a conformational change induced by S-nitrosylation (Bolotina et al., 1994). Similar results have been found for the voltage-gated calcium channels in vascular smooth muscle (Xu et al., 1998). More recently, poly-S-nitrosylation has been identified as a mechanism for the activation of cardiac and skeletal muscle calcium release channels (ryanodine receptors) (Stoyanovsky et al., 1997; Xu et al., 1998). 


\subsubsection{Energy Metabolism}

Early studies on macrophage-induced injury of neoplastic cells (Granger \& Lehninger, 1982; Granger et al., 1980) suggested that endogenously produced NO was a potent inhibitor of mitochondrial respiration and energy metabolism (Hibbs $e t$ al., 1990; Stuehr \& Nathan, 1989). It is now known that NO is capable of inhibiting mitochondrial cis-aconitase, and complexes I and II of the electron transport chain (Hibbs et al., 1990; Stuehr \& Nathan, 1989), although there is some controversy as to whether peroxynitrite is the actual modifying species. These enzymes all contain ironsulphur clusters and the method of inhibition seems to be the displacement of iron from these centers. This process has been shown to be readily reversible by the addition of iron salts (Hibbs et al., 1990) and hence would be suited to a role in physiological regulation controlling oxygen utilization. Glyceraldehyde-3-phosphate dehydrogenase (GAPDH) is an important enzyme in the glycolysis pathway and has been shown to be inhibited by NO in rat liver (Mohr et al., 1994; Molina y Vedia et al., 1992). The inhibition appears to be by direct S-nitrosation and would predictably suppress anaerobic ATP generation. NO has also been shown to stimulate the apparent ADP-ribosylation of GAPDH in the presence of NAD+ or NADH. This is an inductive automodification, independent of endogenous ADP-ribosyltransferase, and involves an active site cysteine (Dimmeler \& Brune, 1992). S-nitrosylation of this residue leads to ADP-ribosylation-induced inhibition of catalytic activity and the depression of glycolysis and gluconeogenesis. This active site thiol of GAPDH is readily oxidized under oxidative stress conditions $\left(\mathrm{H}_{2} \mathrm{O}_{2}\right)$ leading to irreversible inhibition by disulphide generation or oxidant-specific S-thiolation (SchuppeKoistinen et al., 1994). Therefore, S-nitrosylation may represent a mechanism to 
protect GAPDH from irreversible inhibition in this manner, perhaps regulating glucose metabolism (Mohr et al., 1996).

Intracellular energy stores can also be depleted by the enzyme poly-ADP ribosyl transferase (PARP) in a process activated by DNA strand breaks. PARP catalyzes the addition of long chains of NAD to the DNA strand break ends and hence depletes intracellular NADH stores. NADH would normally be used to generate ATP by oxidative phosphorylation and this process leads to cell death by energy store depletion. NO has been shown to activate PARP and to contribute to neurotoxicity in vitro (Zhang et al., 1994).

\subsubsection{Transcription Factors and DNA Synthesis}

NO has been shown to inhibit cell proliferation (Hibbs et al,, 1990; Stuehr \& Nathan, 1989) and this could be due to the previously discussed effects on energy metabolism and ATP synthesis. However, the anti-proliferative effect occurs prior to inhibition of ATP synthesis and most likely occurs by an alternate mechanism (Hibbs et al., 1990). The rate-limiting process for cell division is the synthesis of deoxyribonucleotides from ribonucleotides, a reaction catalyzed by ribonucleotide reductase. It has been demonstrated that NO can interact with the iron-sulphur cluster of this enzyme and this may be a possible method of enzyme inactivation. Studies show that the inhibition of DNA synthesis occurs simultaneously with NO production and this inhibition can be prevented by selective NOS inhibitors (Kwon et al., 1991). Also, adenocarcinoma cells overexpressing ribonucleotide reductase have been shown to be much less sensitive to NO-mediated cytotoxicity than wild-type cells (Lepoivre et al., 1990). 
In addition to these more general effects on DNA synthesis, NO can influence gene expression by interaction with transcription factors. Many transcription factors contain zinc fingers, zinc complexed with thiol ligands of cysteine and/or imidazole nitrogen atoms of histidine. Redox sensitive transcription factors, including Sp1 and Egr-1 (O'Halloran, 1993), bind specific DNA sites via three zinc fingers of the $\mathrm{Cys}_{2} \mathrm{His}_{2}$ type (Kadonaga et al., 1987). Zinc is easily displaced from this structure by redox reactions or intracellular thiol compounds (Zeng et al., 1991). NO can also interact with $\mathrm{Zn}$-sulfur clusters in transcription factors, such as the LAC9-containing Cys $_{6} \mathrm{Zn}(\mathrm{II})$-type cluster (Kroncke et al., 1994). It has also been demonstrated that NO can modulate the DNA-binding capabilities of other transcription factors by Snitrosylation, these include NF-KB p50 and c-jun (dela Torre et al., 1998). These findings reveal that NO may be capable of altering the structural integrity of transcription factors and thereby influence the expression of specific genes.

\subsubsection{Iron Metabolism}

Iron release has been shown to be an important effector of tumor cell cytotoxicity by activated rodent macrophages (Hibbs et al., 1990) and limitation of cell growth by iron availability is ubiquitous in nature. High concentrations of NO, especially in the presence of superoxide, can profoundly disturb iron metabolism, leading to a substantial loss of intracellular iron (Hibbs et al., 1990). Free intracellular iron contributes to cell toxicity via the formation of highly reactive oxygen species by the fenton reaction (Gutteridge, 1989) and NO may contribute to this by causing the release of iron from ferritin, an iron storage protein (Reif \& Simmons, 1990). 
More recently NO has been shown to influence intracellular iron metabolism at the post-transcriptional level by direct interaction with cytosolic aconitase (also referred to as the iron-regulatory factor, IRF). NO disrupts the conformation of the iron-sulfur cluster at the catalytic site of the enzyme and increases the binding of IRF to a specific mRNA structure (the iron-responsive element, IRE) (Drapier et al., 1993; Pantopoulos \& Hentze, 1995; Weiss et al., 1993). This element is present in mRNA transcripts of numerous proteins involved in iron homeostasis. These include ferritin, transferrin receptor, erythroid 5-aminolevulinate synthase and mitochondrial aconitase. Binding of IRF to the IRE of ferritin mRNA inhibits ribosomal binding and represses protein expression. In the case of transferrin receptor mRNA, the same process protects the transcript from RNAse digestion and increases mRNA half-life, resulting in increased protein synthesis. Hence the interaction between NO and iron may regulate cellular iron by direct interaction with storage proteins and by translational control of the various proteins involved in iron homeostasis. NO may also contribute to cellular toxicity by its effects on iron metabolism (Drapier \& Hibbs, 1988; Hibbs et al., 1988; Schwarz et al., 1995).

\subsubsection{Apoptosis}

Apoptosis, the process of cell suicide, is conserved in all animal species and represents a critical feature of the regulated development of multicellular organisms. Apoptotic cell death is characterized by a distinct set of cellular changes, including cell shrinkage, DNA fragmentation, chromatin condensation and membrane blebbing (Cohen, 1993). Apoptosis can result from the developmentally controlled activation of endogenous execution programs (Ellis et al., 1991) or from the transduction of death signals triggered by a variety of exogenous stimuli (Steller, 1995). The process 
is regulated by various "death genes", including the Bcl-2-like proteins and the caspase family of proteases. The role of NO in this process is controversial, with it being implicated in both induction and suppression of apoptosis (for review see Dimmeler \& Zeiher, 1997).

NO can induce the biochemical features of apoptosis in a variety of cells, including macrophages (Albina et al., 1993; Messmer et al., 1996; Sarih et al., 1993), pancreatic $\beta$-cells (Ankarcrona et al., 1994; Kaneto et al., 1995)and thymocytes (Fehsel et al., 1995). Also, transfection of the iNOS gene has been demonstrated to cause apoptosis in vascular smooth muscle cells (Iwashina et al., 1998). The proapoptotic effects of NO seem to be mediated in a guanylate cyclase-independent manner (Brune et al., 1996). NO may act directly to damage DNA by deamination reactions (Inoue \& Kawanishi, 1995; Nguyen et al., 1992), and hence induce apoptosis (Nguyen et al., 1992). Also, accumulation of the cell death gene product, P53, has been described as an early indicator of NO-induced apoptosis (Messmer et al., 1994). Subsequent activation of caspases leads to the proteolysis, and activation of PARP (Messmer et al., 1996). This is supported by the finding that overexpression of Bcl-2 (Messmer et al., 1996) or HSP70 (Bellmann et al., 1996) abrogates NOinduced apoptosis and PARP cleavage, but not p53 accumulation. There may also be p53-independent pathways (Messmer \& Brune, 1996) for the activation of apoptosis by $\mathrm{NO}$ and this indicates that the proapoptotic signal transduction by NO may be more much more complex than suggested above.

The precise NO species involved in the induction of apoptosis remains unclear. Studies have indicated that the proapoptotic effects of NO are due to the 
formation of peroxynitrite and this species has been shown to induce apoptosis in thymocytes (Salgo et al., 1995), neuronal cells, and HL-60 cells (Estevez et al., 1995; Lin et al., 1995) . However, studies using physiological concentrations of peroxynitrite found no evidence of the induction of apoptosis in endothelial or mononuclear cells (Lin et al., 1995). Also, the simultaneous production of oxygen radicals and NO, conditions ideal for the production of peroxynitrite, had less of an apoptotic effect than NO alone (Brune et al., 1997; Sandau et al., 1997). Hence it seems most likely that NO and not peroxynitrite is the effective compound which induces DNA damage by deamination of DNA.

There are several ways in which NO has been shown to inhibit apoptosis. The precise mechanisms are unclear, but a number of molecular targets have been identified. One key action of NO may be to increase cGMP levels, via activation of guanylate cyclase. However, the role of cGMP in the control of apoptotic signals is still controversial. Some studies demonstrated that NO could prevent apoptosis in human eosinophils during cytokine deprivation (Beauvais et al., 1995) and that these effects could be mimicked by stable cGMP analogues (Beauvais et al., 1995; Chun et al., 1995; Mannick et al., 1994). Conversely, other studies did not reveal any protective effect of cGMP analogues (Dimmeler et al., 1997; Genaro et al., 1995; Kim et al., 1997). Since NO-induced cGMP elevation is short lived, an interference with apoptosis signal transduction, requiring at least 3-24 hours, appears to be highly unlikely under normal physiological conditions. Further research in this area is required to resolve this controversy. 
NO has also been shown to have a direct inhibitory effect on the caspase cascade, the activation of caspases by proteolysis of inactive precursors into active subunits. It has recently been shown that endothelial-derived NO, and low micromolar concentrations of exogenous NO donors, can inhibit TNF- $\alpha$-induced caspase activation in endothelial cells (Dimmeler et al., 1997). Inhibition of a specific caspase family member, caspase-3, has been shown to be due to specific S-nitrosylation of Cys 163, a functionally essential amino acid which is conserved throughout the caspase protein family (Dimmeler et al., 1997). Indeed NO has been shown to inhibit at least seven members of the caspase family in vitro, probably by S-nitrosylation ( $\mathrm{Li}$ et al., 1997). In addition to the role of caspases in TNF- $\alpha$ and FAS/APO-1 induced apoptosis, they also turn out to be of general importance in the apoptotic signaling cascade (Enari et al., 1996; Los et al., 1995; Nicholson et al., 1995). Low level NO may serve to modulate caspase activity and may control the responses of cells to death signals.

NO has also been implicated in the control of Bcl-2, expression of this antiapoptotic protein has been shown to be enhanced by NO in B-cells (Genaro et al., 1995). More recent experiments have demonstrated a role for NO in the posttranslational regulation of $\mathrm{Bcl}-2$ by inhibition of TNF- $\alpha$-induced proteolysis (Dimmeler et al., 1998). This could lead to sustained levels of Bcl-2 and a reduction in sensitivity to apoptotic signals. NO may also induce the expression of other antiapoptotic proteins, such as heat shock proteins. It has been reported that NO can induce the expression of HSP32 and HSP70, thereby preventing TNF- $\alpha$ /actinomycin D induced apoptosis in hepatocytes (Xu et a., 1998; Rettori et al., 1993). However, the mechanism of this protective effect of HSPs is unclear. It is clear from the above 
that NO and/or NO-derived species, can either elicit or suppress apoptosis depending on concentration, the biological redox environment, the induction of intracellular defense mechanisms, the apoptotic stimulus and the cell type involved.

\subsubsection{Direct Cytotoxicity and Cell Defense Mechanisms}

Sensitivity toward the cytotoxic action of NO varies greatly in different mammalian cell types and cell lines (Kroncke et al., 1993). Islet cells (Kroncke et al., 1991) and neurons are extremely sensitive to the toxic actions of macrophage derived NO and NO-donor compounds (Boje \& Arora, 1992; Chao et al., 1992). Other cell types, such as human keratinocytes and rat aorta endothelial cells are much less sensitive (Kroncke et al., 1993). Differences in sensitivity may be due to differing capacities of cells for NO scavenging, detoxification, the efficiency of anaerobic respiration and the efficacy of DNA repair mechanisms. Glutathione is the most dominant cellular antioxidant; and reacts with NO to form S-nitrosoglutathione (Clancy et al., 1994). Depletion of glutathione stores has been shown to dramatically enhance NO-mediated cytotoxicity, indicating an important NO-scavenging role of this compound (Walker et al., 1995). However, the S-nitrosoglutathione is rapidly broken down, by the intracellular thioredoxin system (Nikitovic \& Holmgren, 1996) and glutathione peroxidase (Hou et al., 1996), to release NO. Hence it is more likely that NO sensitivity is dependent upon the relative activity of the broader cellular antioxidant system. This consists of catalase, superoxide dismutases (SODs), glutathione reductase, glutathione peroxidase, thioredoxin, thioredoxin reductase and the capacity to supply reduction equivalents via the hexose monophosphate shunt (Clancy et al., 1994). Expression of some of these proteins can be influenced by NO at the transcriptional level. NO has been shown to induce the expression of Mn-SOD 
(Sano et al., 1996), heme oxygenase (Motterlini et al., 1996), and stress activated protein kinases (Pfeilschifter \& Huwiler, 1996). In addition, a cell line that overexpresses metallothionein was found to be relatively resistant to NO cytotoxicity (Schwarz et al., 1995).

Macrophage-derived NO has been shown to be a cytotoxic effector against tumor cells by the previously discussed effects on intracellular iron, DNA synthesis and energy metabolism (Drapier \& Hibbs, 1988; Wharton et al., 1988). NO and derived species can also contribute to it's own toxicity by incapacitating some of the defense mechanisms discussed above. Thus peroxynitrite has been shown to interact with the transition metal in the active site of (SOD) giving rise to a nitronium ion. This results in self-nitration of tyrosine residues and inactivation of both the Mn- and Fe-SOD, but not Cu/Zn-SOD (Ischiropoulos et al., 1992). NO can also inhibit glutathione reductase by nitrosylation of cysteines located in the active site of this

enzyme (Wharton et al., 1988). NO has also been shown to inhibit metallothionein by interaction with the iron-sulfur (Kennedy et al., 1993; Schwarz et al., 1995) and catalase by interaction with the heme group (Brown, 1995).

\subsubsection{Other Targets}

NO has also been demonstrated to have a modulating effect on methionine metabolism in rats by inhibition of hepatic methionine adenosyltransferase via Snitrosylation (Ruiz et al., 1998). Alcohol metabolism has been shown to be modulated by NO via S-nitrosylation-dependent inhibition of alcohol dehydrogenase (Gergel \& Cederbaum, 1996). Caeruloplasmin is a copper containing protein in the 
plasma with ferroxidase activity. Peroxynitrite interacts with this protein resulting in copper release and the loss of ferroxidase activity (Swain et al., 1994).

\subsubsection{Summary}

NO is simultaneously involved in signaling, cytotoxicity, and cytoprotection by interaction with a variety of molecules present in the intracellular environment. The resulting redox family of NO-derived compounds with their range of reactivity and diversity of intracellular targets account for the broad biological actions of NO. Such complexity makes the therapeutic remediation of conditions that arise from excess or insufficient NO a great challenge, but one with the potential for even greater rewards.

\subsection{Nitric Oxide Synthases}

Mammalian nitric oxide synthases (NOSs) have been characterized from various cell types and found to comprise three distinct isoforms that are $50-60 \%$ homologous and distinguished by their histological expression, susceptibility to arginine-based inhibitors, intracellular localization, NO output and mode of regulation (for review see Sessa, 1994). Within each isoform group there is a high degree of conservation of amino acid sequence (80-94\%) across species, demonstrating that these differences are species-related (orthologous) and not due to the existence of different genes. These isoforms are products of three distinct genes (Chartrain et al., 1994; Hall et al., 1994; Kishimoto et al., 1992; Marsden et al., 1994; Marsden et al., 1993; Robinson et al., 1994; Xu et al., 1994; Xu et al., 1993) and are functionally categorized as to whether their expression is constitutive or inducible (see tables 1.2 and 1.3). 
Two isoforms, approximately $60 \%$ homologous, are constitutively expressed in specific cell types where activity is regulated by changing levels of intracellular $\mathrm{Ca}^{2+}$ (cNOSs). The two cNOSs are termed neuronal NOS (nNOS $\approx 160 \mathrm{kDa}$ ) and endothelial NOS (eNOS $\approx 130 \mathrm{kDa}$ ), named for the tissues from which they were initially isolated. These cNOSs are transiently activated by agonist-induced elevations in intracellular calcium, resulting in the evanescent binding of $\mathrm{Ca}^{2+} / \mathrm{calmodulin}$. This pulsatile activation is ideally suited to a regulatory role by puffs of NO in cells and tissues.

The remaining isoform, iNOS $(\approx 130 \mathrm{kDa})$, can be induced by immunostimulants in most cell types; activity is continuous, high output and $\mathrm{Ca}^{2+}-$ independent. iNOS was originally isolated from murine macrophages as an enzyme that contains tightly-bound calmodulin (Cho et al., 1992) and accordingly, it is fully active at resting low levels of intracellular calcium. iNOS produces a large continuous flux of NO which may be limited only by substrate availability. Transcriptional upregulation of the iNOS gene occurs in response to various inflammatory cytokines (e.g. IL-1, TNF $\alpha$ ) or immunostimulants such as bacterial lipopolysaccharide (LPS), by a mechanism that is synergistic with interferon- $\gamma$ (for review see Nathan \& Xie, 1994). While the high-output of NO that derives from iNOS plays an important role in host-defense against intracellular parasitism (Green et al., 1990; Hayashi et al., 1996), it can also be toxic to the host, contributing to inflammatory dysfunctions and potentially lethal hypotension (Kilbourn et al., 1990; Kilbourn et al., 1992; Kilbourn et al., 1994; Lin et al., 1994; Liu et al., 1997; Petros 
et al., 1991). Evidence suggests that iNOS may be expressed in some tissues under normal conditions; these include fetal and adult lung (Kobzik et al., 1993) and kidney. This possibility challenges the common view that iNOS is exclusively inducible. An overall comparison of the NOS isoforms is shown in Fig. 1.1

\subsection{Nitric Oxide Synthase: Gene Structure, Regulation and Expression}

Three distinct genes encoding the family of human NOS proteins have been identified and characterized. Analysis of these complex loci reveals that the control of mRNA expression is unique for each of the different NOS isoform genes. The NOS genes are not clustered in one part of the genome, but are spread out on separate chromosomes. Analysis of the genomic organization and exon/intron structure indicates a remarkably high degree of similarity between the three genes. This is especially evident in the two $\mathrm{Ca}^{2+} /$ calmodulin-dependent NOSs (Hall et al., 1994; Marsden et al., 1993). The high degree of conservation between the NOS isoforms may be due to evolutionary events such as gene duplication and chromosome transposition.

The three genes share several striking features, they all display the same exon structure, discounting the first exons, which supports the theory that the three genes are probably derived from a common ancestral gene. Most of the exon sizes are similar and the position of the introns within interrupted codons are always the same. However, there are two important differences between the cNOS genes and the iNOS gene. Firstly, nNOS exon 15 and eNOS exon 17 have no counterpart in iNOS. Secondly, iNOS exon 12 corresponds to two exons in both nNOS (exons 12 and 13) 
Table 1.2 Purified Nitric Oxide Synthases

\begin{tabular}{|c|c|c|c|c|c|}
\hline Species and Tissue & Classification & $\begin{array}{l}\text { Co-factor } \\
\text { additions required } \\
\text { for activity }\end{array}$ & $\mathrm{M}_{\mathrm{r}}(\mathrm{KDa})$ & Specific Activity & Reference \\
\hline Rat Cerebellum & $\begin{array}{l}\text { Constitutive } \\
\text { Soluble }\end{array}$ & $\begin{array}{l}\mathrm{Ca}^{2+} / \mathrm{CaM} \\
\mathrm{NADPH}\end{array}$ & 150 & $\begin{array}{l}960 \mathrm{nMol} \text { citrulline } \\
/ \mathrm{min} / \mathrm{mg} \text { protein }\end{array}$ & Bredt and Snyder, 1990 \\
\hline Rat Cerebellum & $\begin{array}{l}\text { Constitutive } \\
\text { Soluble }\end{array}$ & $\begin{array}{l}\mathrm{Ca}^{2+} / \mathrm{CaM} \\
\mathrm{NADPH}\end{array}$ & 155 & $\begin{array}{l}107 \mathrm{nMol} \text { citrulline } \\
/ \mathrm{min} / \mathrm{mg} \text { protein }\end{array}$ & Schmidt et al, 1991 \\
\hline Rat Cerebellum & $\begin{array}{l}\text { Constitutive } \\
\text { Particulate }\end{array}$ & $\begin{array}{l}\mathrm{Ca}^{2+} / \mathrm{CaM}, \\
\mathrm{NADPH}, \mathrm{FAD}, \\
\mathrm{BH}_{4}\end{array}$ & 150 & $\begin{array}{l}20 \mathrm{nMol} \text { citrulline } \\
/ \mathrm{min} / \mathrm{mg} \text { protein }\end{array}$ & Hiki et al, 1992 \\
\hline Porcine Cerebellum & $\begin{array}{l}\text { Constitutive } \\
\text { Soluble }\end{array}$ & $\begin{array}{l}\mathrm{Ca}^{2+} / \mathrm{CaM} \\
\mathrm{NADPH}, \mathrm{BH}_{4}\end{array}$ & 160 & $\begin{array}{l}730 \mathrm{nMol} \text { citrulline } \\
/ \mathrm{min} / \mathrm{mg} \text { protein }\end{array}$ & Mayer et al, 1990 \\
\hline Human cerebellum & $\begin{array}{l}\text { Constitutive } \\
\text { Soluble }\end{array}$ & $\begin{array}{l}\mathrm{Ca}^{2+} / \mathrm{CaM} \\
\mathrm{NADPH}, \mathrm{BH}_{4}\end{array}$ & 160 & $\begin{array}{l}74.3 \mathrm{nMol} \text { citrulline } \\
/ \mathrm{min} / \mathrm{mg} \text { protein }\end{array}$ & Schmidt and Murad, 1991 \\
\hline Bovine Brain & $\begin{array}{l}\text { Constitutive } \\
\text { Soluble }\end{array}$ & $\begin{array}{l}\mathrm{Ca}^{2+} / \mathrm{CaM} \\
\mathrm{NADPH}, \mathrm{BH}_{4}\end{array}$ & 150 & $\begin{array}{l}115 \mathrm{nMol} \text { citrulline } \\
/ \mathrm{min} / \mathrm{mg} \text { protein }\end{array}$ & Ohshima et al, 1992 \\
\hline $\begin{array}{l}\text { Bovine aortic } \\
\text { endothelial cells }\end{array}$ & $\begin{array}{l}\text { Constitutive } \\
\text { Particulate }\end{array}$ & $\begin{array}{l}\mathrm{Ca}^{2+} / \mathrm{CaM}, \\
\mathrm{NADPH}, \mathrm{BH}_{4}\end{array}$ & 135 & $\begin{array}{l}15.3 \mathrm{nMol} \text { citrulline } \\
/ \mathrm{min} / \mathrm{mg} \text { protein }\end{array}$ & Pollock et al, 1991 \\
\hline Rat macrophage & $\begin{array}{l}\text { Inducible } \\
\text { Soluble }\end{array}$ & $\begin{array}{l}\mathrm{NADPH}, \mathrm{BH}_{4}, \\
\text { DTT }\end{array}$ & 150 & $\begin{array}{l}1052 \mathrm{nMol} \text { citrulline } \\
/ \mathrm{min} / \mathrm{mg} \text { protein }\end{array}$ & Yui et al, 1991 \\
\hline
\end{tabular}




\begin{tabular}{|c|c|c|c|c|c|}
\hline Mouse Macrophage & $\begin{array}{l}\text { Inducible } \\
\text { Soluble }\end{array}$ & $\begin{array}{l}\text { NADPH, } \mathrm{BH}_{4}, \\
\text { FAD }\end{array}$ & 130 & $\begin{array}{l}0.96 \mathrm{nmol} \\
\text { citrulline/hr }\end{array}$ & Hevel et al, 1991 \\
\hline Mouse Macrophage & $\begin{array}{l}\text { Inducible } \\
\text { Soluble }\end{array}$ & $\begin{array}{l}\text { NADPH, } \mathrm{BH}_{4}, \\
\text { FAD, thiol }\end{array}$ & $125-135$ & $\begin{array}{l}1313 \mathrm{nmol} \mathrm{NO}_{\mathrm{x}} \\
/ \mathrm{min} / \mathrm{mg} \text { protein }\end{array}$ & Stuehr et al, 1991 \\
\hline Rat Liver & $\begin{array}{l}\text { Inducible } \\
\text { Soluble }\end{array}$ & $\begin{array}{l}\mathrm{CaM}, \mathrm{BH}_{4}, \mathrm{FAD} \text {, } \\
\mathrm{FMN}\end{array}$ & 135 & $\begin{array}{l}462 \mathrm{nMol} \text { citrulline } \\
/ \mathrm{min} / \mathrm{mg} \text { protein }\end{array}$ & Evans et al, 1992 \\
\hline $\begin{array}{l}\text { Rat } \\
\text { polymorphonuclear } \\
\text { neutrophils }\end{array}$ & $\begin{array}{l}\text { Inducible } \\
\text { Soluble }\end{array}$ & $\begin{array}{l}\mathrm{Ca}^{2+}, \mathrm{NADPH} \\
\mathrm{FAD}, \mathrm{BH}_{4}\end{array}$ & 150 & $\begin{array}{l}122 \mathrm{nmol} \mathrm{NO} \mathrm{x}_{\mathrm{x}} \\
/ \mathrm{min} / \mathrm{mg} \text { protein }\end{array}$ & Yui et al, 1991 \\
\hline
\end{tabular}


Table 1.3. Cloned Nitric Oxide Synthase Isoforms

\begin{tabular}{|c|c|c|c|c|c|}
\hline NOS Isoform & Species & Cell Type & $\begin{array}{l}\text { Protein } \mathrm{M}_{\mathrm{r}} \\
\text { (Deduced) }\end{array}$ & $\begin{array}{l}\mathrm{Ca}^{2+}- \\
\text { dependent }\end{array}$ & Reference \\
\hline $\begin{array}{l}\text { Neuronal } \\
\text { (NOS 1) }\end{array}$ & $\begin{array}{l}\text { Human } \\
\text { Rat }\end{array}$ & $\begin{array}{l}\text { Cerebellum } \\
\text { Cerebellum }\end{array}$ & $\begin{array}{l}161037 \mathrm{Da} \\
160458\end{array}$ & $\begin{array}{l}\text { Yes } \\
\text { Yes }\end{array}$ & $\begin{array}{l}\text { Nakane et al., } 1993 \\
\text { Bredt et al., } 1991\end{array}$ \\
\hline $\begin{array}{l}\text { Inducible } \\
\text { (NOS 2) }\end{array}$ & $\begin{array}{l}\text { Human } \\
\text { Rat } \\
\text { Rat } \\
\text { Murine } \\
\end{array}$ & $\begin{array}{l}\text { Hepatocyte } \\
\text { Hepatocyte } \\
\text { Vascular Smooth } \\
\text { Muscle } \\
\text { Macrophage } \\
\end{array}$ & \begin{tabular}{|l|}
131000 \\
130653 \\
131000 \\
130556 \\
\end{tabular} & $\begin{array}{l}\text { No } \\
\text { No } \\
\text { No } \\
\text { No } \\
\end{array}$ & $\begin{array}{l}\text { Gellar et al., } 1993 \\
\text { Wood et al., } 1993 \\
\text { Nunokawa et al., } 1993 \\
\text { Lowenstein et al., } 1992\end{array}$ \\
\hline $\begin{array}{l}\text { Endothelial } \\
\text { (NOS 3) }\end{array}$ & $\begin{array}{c}\text { Human } \\
\text { Bovine }\end{array}$ & $\begin{array}{l}\text { Aortic endothelium } \\
\text { Aortic endothelium }\end{array}$ & $\begin{array}{r}133000 \\
133286\end{array}$ & $\begin{array}{l}\text { Yes } \\
\text { Yes }\end{array}$ & $\begin{array}{l}\text { Janssens et al., } 1992 \\
\text { Marsden et al., } 1992 \\
\text { Lamas et al., } 1992 \\
\text { Nishida et al., } 1992 \\
\text { Sessa et al., } 1992\end{array}$ \\
\hline
\end{tabular}




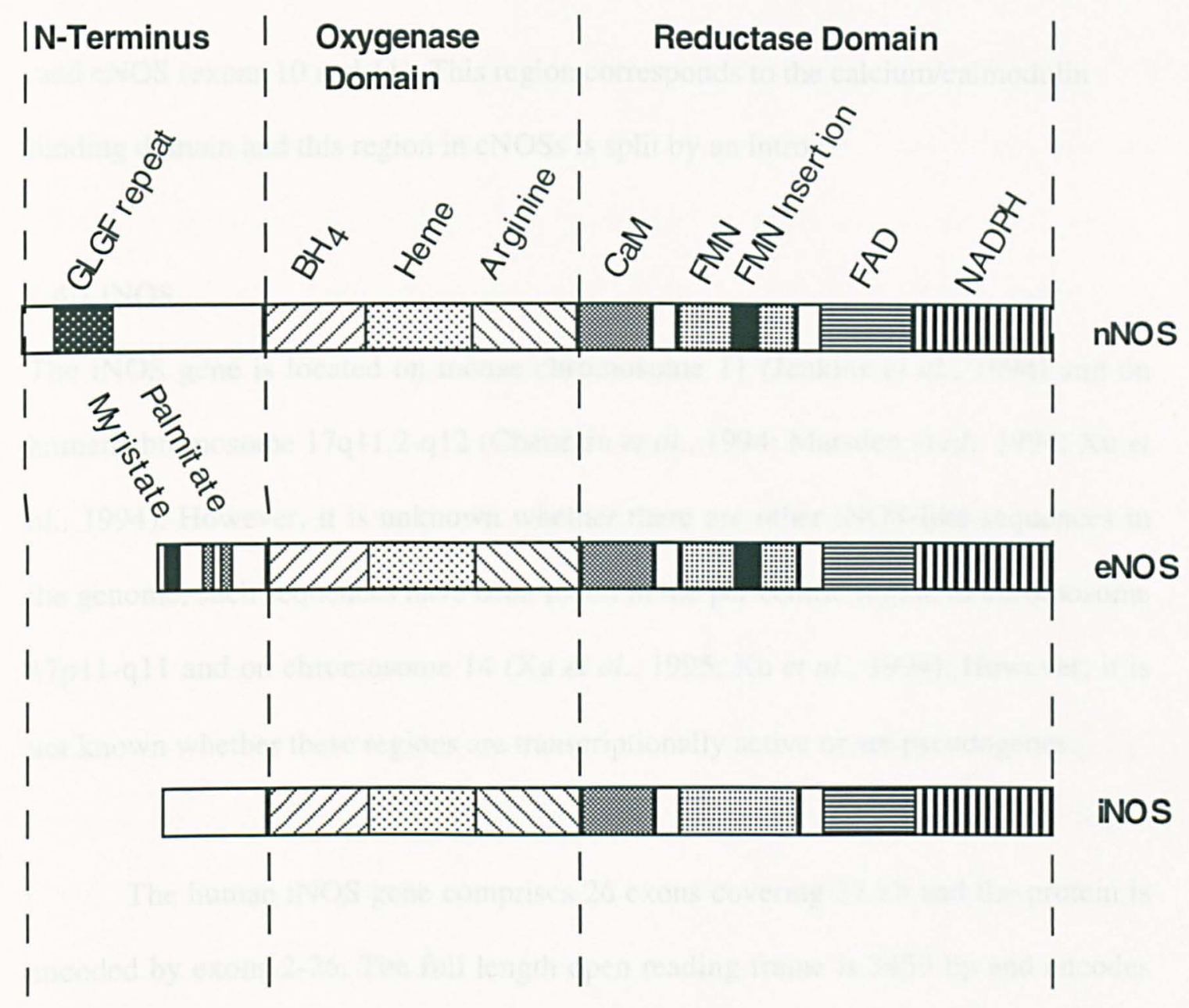

Fig 1.1 Overview and Comparison of the Modular Structure of Nitric Oxide Synthase Isoforms

The diagram shows the division of NOSs into distinct reductase and oxygenase domains. Special note should be made of the lack of homology in the N-terminal regions, with nNOS and eNOS containing important posttranslational modification sites within this area. nNOS contains a GLGF repeat and eNOS contains acylation sites which are important in the subcellular localization of the proteins necessary for efficient in vivo function of the enzymes. 
and eNOS (exons 10 and 11). This region corresponds to the calcium/calmodulin binding domain and this region in cNOSs is split by an intron.

\subsection{1 iNOS}

The iNOS gene is located on mouse chromosome 11 (Jenkins et al., 1994) and on human chromosome 17q11.2-q12 (Chartrain et al., 1994; Marsden et al., 1994; Xu et al., 1994). However, it is unknown whether there are other iNOS-like sequences in the genome, such sequences have been found in the pericentric region of chromosome 17p11-q11 and on chromosome 14 (Xu et al., 1995; Xu et al., 1994). However, it is not known whether these regions are transcriptionally active or are pseudogenes.

The human iNOS gene comprises 26 exons covering $37 \mathrm{~kb}$ and the protein is encoded by exons $2-26$. The full length open reading frame is $3459 \mathrm{bp}$ and encodes for a protein of 1153 amino acids $(130 \mathrm{kDa})$. A transcription initiation site 30 nucleotides downstream of a TATA box has been identified in studies using cytokine/LPS-stimulated human hepatocytes (Chartrain et al., 1994). Analysis of the 5'-flanking region shows several consensus sequences that are implicated in cytokinemodulated gene expression. These include NF- $\kappa B$, NF-interleukin- 6 (IL-6), $\gamma$-IRE, a palindromic TNF-RE-like site, and a liver-specific transcription factor consensus sequence, AABS (Chartrain et al., 1994; Nunokawa et al., 1994).

The expression of iNOS can be induced in almost every tissue and nucleated cell type tested thus far. The induction of iNOS expression in these varied cell types appears to be predominantly under transcriptional regulation. Although there is a low basal level of expression, this is markedly enhanced by treatment of cells with LPS or 
cytokines. Maximal promoter activation occurs with synergistic combinations of inflammatory cytokines (TNF- $\alpha$, IL-1 $\beta$, IFN- $\gamma$ ) and LPS. What constitutes an effective combination varies depending on cell-type and species (Beasley \& Eldridge, 1994; Geller et al., 1993; Lorsbach et al., 1993; MacNaul \& Hutchinson, 1993; Markewitz et al., 1993; Sherman et al., 1993; Sirsjo et al., 1994; Suschek et al., 1993; Wood et al., 1993; Yamada et al., 1993). Activation of protein kinase A (PKA), protein kinase $\mathrm{C}(\mathrm{PKC})$ and protein tyrosine kinase signaling pathways have been implicated in a modulatory role in some cell types (Gilbert \& Herschman, 1993). Characterization of the murine iNOS promoter indicates that distinct areas of the 5'flanking regions of the gene participate in the transcriptional response to the different exogenous stimuli (Lowenstein et al., 1993; Xie et al., 1994; Xie et al., 1993). There is a proximal region of the promoter $(-85 /-76)$ which interacts with the NF-KB transacting factor. NF- $\mathrm{kB}$ resides in the cytosol of quiescent cells as an inactive complex, bound to an inhibitory protein, I-KB. Following activation of cells by a number of agents, I- $\mathrm{KB}$ becomes phosphorylated, dissociates and is degraded by the proteosome. This allows NF- $\mathrm{kB}$ to translocate to the nucleus, where it binds to the cis-regulatory regions of the iNOS promoter (Grilli et al., 1993; Xie et al., 1994). This proximal NF$\kappa \mathrm{B}$ binding site has been shown to be critical for the induction of the iNOS gene by LPS (Lowenstein et al., 1993; Xie et al., 1993). Promoter/reporter construct studies indicate that the more distal regions of the promoter are involved in IFN- $\gamma$-stimulated changes in iNOS mRNA expression (Lowenstein et al., 1993; Xie et al., 1993). An IRF-1 cis-regulatory DNA region is implicated in this response and this is supported by in vivo evidence demonstrating that homozygous IRF-1 (-/) mice do not exhibit an iNOS response following stimulation by cytokines or viruses (Kamijo et al., 1994). 


\section{$1.4 .2 \mathrm{nNOS}$}

The nNOS gene is present as a single copy in the haploid human genome and is located on human chromosome 12q24.2-24.31 (Kishimoto et al., 1992; Marsden et al., 1994; Xu et al., 1993). The gene comprises 28 codons, 2-29 coding for the protein and spanning over $200 \mathrm{~kb}$. The nNOS gene yields an mRNA with an open reading frame of $4302 \mathrm{bp}$ that encodes a protein of 1434 amino acids with a predicted molecular weight of $160.8 \mathrm{kDa}$. Homologous genes of mouse and rat have been assigned to chromosome 5 (Lee et al., 1995) and chromosome 12 (Deng et al., 1995), respectively. The genomic structure for human brain nNOS is well documented (Hall et al., 1994; Marsden et al., 1994). More recently sequence data for the rat nNOS transcriptional cluster expressed in yeast artificial chromosome has been published (Lee et al., 1997). Also nNOS cDNAs have been isolated for both rat and mouse homologues (Bredt et al., 1991; Ogura et al., 1993), and the coding sequences show $86-88 \%$ homology to the human gene at the nucleotide level.

Consensus sequences coding for the various functional domains of the protein are localized to distinct exons or groups of exons. Although considered to be constitutively expressed, nNOS may also be transcriptionally regulated. This is based on the fact that the $5^{\prime}$-flanking region of the human nNOS exon 1 contains putative transcription factor binding sites for AP-2, TEF-1/MCBF, CREB/ATF/c-fos, NRF-1. Ets, NF-1, and NF-kB-like sequences (Hall et al., 1994). However, these sites have not been proven to have functional activity with transcription factors. In addition, a variety of cis-regulatory elements implicated in testis-specific transcription are evident including the transcription factor GATA and GATA-like sites, p53 halfelement, an Ets binding site, Pu box, polyomavirus enhancer activator 3 (PEA3) 
sequences, myocyte-specific enhancer factor 2 motif, and an insulin response site. Again the functionality of these sites has not been proven. The transcription initiation sites in brain nNOS mRNA has been determined via primer extension analysis and RNAse protection, revealing a major start site 28 nucleotides downstream of a TATA box.

Cloning and characterization of nNOS cDNAs from both human and rodent tissues reveals structural and alleic mRNA diversity. Various broadly distributed, tissue-specific, or developmentally regulated mRNA transcripts have been reported. These arise from combinations of two or three of the following mechanisms. Initiation by different transcriptional units containing alternative promoters, cassette exon deletions or insertions, and the use of polyadenylation signals. The usage of alternative promoters seems to be one of the main mechanisms involved in controlling nNOS gene expression, in a tissue specific manner. For the human gene two major transcriptional clusters have been identified, neuronal- and testis-specific. The neuronal cluster is located upstream from exon two. At least eight different first exons and the corresponding 5'-flanking promoter regions have been detected within the $200 \mathrm{~kb}$ gene sequence. Major transcription sites were found located at $\geq 25 \mathrm{~kb}$ upstream from exon 2 (Hall et al., 1994; Wang \& Marsden, 1995; Xie et al., 1995; Young et al., 1995). The different first exons appear to splice to the common exon 2 , which contains the initiator ATG codon. Hence, the protein encoded by the different mRNA species is the same, full-length, wild type nNOS protein. However, the use of alternative promoters is a versatile mechanism which can influence gene expression (Ayoubi \& Van De Ven, 1996). These influences of different leader exons influence mRNA processing, localization, translation efficiency, and longevity. The 
various promoters have distinct tissue specificities and developmental regulation and may respond differently to stimuli. This is supported by in situ hybridization studies using antisense cRNAs which localized the different exon 1 variants to distinct cell populations. Results suggest that cell type-specific transcription or splicing factors may control the levels of nNOS expression (Wang \& Marsden, 1995).

Multiple first exons have also been reported for the mouse nNOS gene, cDNA sequencing reveals at least five distinct mRNA transcripts (Brenman et al., 1997) in the rat nNOS gene, with at least three distinct mRNA transcripts arising from alternative splicing (Lee et al., 1997). In normal human testis, transcription can initiate from a region located within intron 3 (Wang et al., 1997). A novel nNOS mRNA transcript with a 5' terminus encoded by two new exons (Tex 1 and Tex 2) spliced to exon 4 of full length nNOS. This appears to be expressed at a comparable level in the testis to that of full length nNOS. Also a minor transcript containing a unique 95bp exon Tex 1b5' of exon 2 was detected. The two mRNAs predicted the translation to occur at an ATG within exon 5, giving rise to a protein (TnNOS) with a calculated molecular mass of $125 \mathrm{kDa}$ (1098 amino acids). This represents an $\mathrm{N}$ terminal truncation of the full length nNOS and lacks the PDZ/GLGF domain. An analogue of TnNOS is found in mice, nNOS- $\gamma$, which is produced by a different molecular mechanism to TnNOS. In nNOS $\Delta / \Delta$ mice with a targeted deletion of exon 2 (Huang et al., 1993), two NOS transcripts $(\beta+\gamma)$ are found at very low levels. These use alternative first exons, $1 \mathrm{a}$ in the case of nNOS- $\beta$ and $1 \mathrm{~b}$ in the case of nNOS- $\gamma$, spliced to a common exon 3 (Brenman et al., 1997). Translation of nNOS- $\gamma$ is initiated at the same ATG within exon 5 as the human TnNOS and gives rise to a 
similar $125 \mathrm{kDa}$ protein. The nNOS- $\beta$ transcript is initiated at an ATG codon within exon 1a, generating a $136 \mathrm{kDa}$ NOS protein with six new $\mathrm{N}$-terminal amino acids.

Different nNOS transcripts can also arise via cassette exon deletions or insertions. Cassette deletions of exon 10 and exons $9 / 10$ have been detected in human nNOS mRNA transcripts (Hall et al., 1994). The deletion of exon 10 introduces a stop codon 16 bp downstream of the splice junction. This could potentially lead to the production of a novel truncated 560 amino acid protein. Excision of exons $9 / 10$ results in a $315 \mathrm{bp}$ frame deletion possibly resulting in the production of a protein lacking 105 amino acids. Low expression of this protein has been shown in various human brain tissues, mouse brain tissues and neuroblastoma cell lines (Fujisawa $e t$ al., 1994; Ogura et al., 1993). It remains to be established that these variants are relevant in vivo, although it is likely since the nNOS-related dNOS cloned from Drosophila exhibits the same 315 bp deletion and is fully active (Regulski \& Tully, 1995).

Cassette exon insertions have also been reported to give rise to novel variants of nNOS. In the rat, a fully active muscle nNOS ( $\mu$ NOS) has been reported to be derived from a $102 \mathrm{bp}$ insertion between exons 16 and 17, resulting in the addition of 34 amino acids (Magee et al., 1996; Silvagno et al., 1996). This is expressed in rat skeletal muscle, heart, penis, urethra and prostate (Magee et al., 1996). In muscle, most of $\mu$ NOS is found associated with dystrophin at the sarcolemma (Chao et al., 1997). Also a cassette insertion of exon Tex 2 between exons 3 and 4 of the full length nNOS has been shown from screening of a human testis cDNA library and was subsequently found in other tissues (Wang et al., 1997). The insertion of 56 bp leads 
to a frameshift, which introduces a stop codon $371 \mathrm{bp}$ downstream of the splice junction within exon 6. The production of the resulting C-terminal truncated, 407 amino acid protein has not been shown to be translated in vivo.

The last source of variation in the nNOS gene is the presence of three potential polyadenylation signals within exon 29 . These sites are present over a 200 bp region with a predominant site located at 6449 bp downstream of the initiator methionine (Hall et al., 1994). Exon 29 also contains a (CA)n repeat sequence and genotypic analysis of normal individuals showed multiple alleles of various sizes, depending on the number of $(\mathrm{CA}) \mathrm{n}$ repeats. The effects of these 3'-untranslated end variations on mRNA transcription efficiency, processing, stability, or subcellular targeting is unknown at present.

\subsection{3 eNOS}

The human eNOS mRNA is encoded by a unique gene consisting of 26 exons spanning 21-22 kb of DNA (Marsden et al., 1993; Miyahara et al., 1994). The human gene has been mapped to the $7 \mathrm{q} 35-7 \mathrm{q} 36$ region of chromosome 7 (Marsden et al., 1993). The bovine eNOS gene also contains 26 exons and spans $20 \mathrm{~kb}$ of DNA. Two transcription start sites are located 170 and 240 bps upstream of the methionine translation initiation codon (Venema et al., 1994). Full length eNOS cDNAs have been isolated from human (Marsden et al., 1992) (Janssens et al., 1992), murine (Gnanapandithen et al, 1996), bovine (Lamas et al., 1992; Nishida et al., 1992; Sessa et al., 1992) and porcine (Zhang et al., 1997) endothelial cells. 
The 5'-flanking region of the bovine eNOS gene indicates that the promoter has no TATA motif and exhibits Sp1 and GATA motifs, elements consistent with a constitutively expressed gene in endothelial cells (Zhang et al., 1995). Analysis of the human eNOS gene promoter reveals consensus sequences for the binding of transcription factors AP-1, AP-2, NF-1, nuclear factor, IL-6, NF-KB and PEA3. Also CACCC-, CCAAT-, heavy metal-, acute-phase response-, shear stress-, cAMPresponse-, retinoblastoma control-, INF- $\gamma$-response-, and sterol-regulatory cis elements appear to be present. Incomplete sites for estrogen response elements have also been identified. So far, a functional response has been shown for only a few of these binding sites and deletion/mutation studies reveal an essential role for the Sp-1 binding site at position -103 (Zhang et al., 1995). Stimulation of the eNOS promoter in the human E.A.hy 926 endothelial cell line has been shown in response to estrogen (Kleinert et al., 1998), and in human umbilical vein endothelial cells (HUVECs) in response to lysophosphatidylcholine (Cieslik et al., 1998); these responses may result from enhanced binding of Sp-1. Mutation of the GATA site at position -230 caused a $30 \%$ reduction in eNOS promoter activity (Zhang et al., 1995) and mutation of the PEA3 binding site at position -26 caused a $50 \%$ reduction in activity. An upregulation of bovine eNOS promoter activity in response to hypoxia has been demonstrated (Arnet et al., 1996), but the human eNOS promoter does not contain any identifiable sites with homology to the hypoxia inducible factor-1 (HIF-1) consensus sequence. A possible role for PKC in the induction of promoter activity has been demonstrated in human endothelial cells transfected with a human eNOS promoter-luciferase construct ( $\mathrm{Li}$ et al., 1998). However, the mechanism and transcription factors involved remain to be elucidated. 
The bovine gene 5 -flanking region, or the known $2.9 \mathrm{~kb}$ of sequence, also lacks a typical TATA box and contains numerous putative transcription factor binding sites. Comparison of the first $1.6 \mathrm{~kb}$ of the 5'-flanking regions of human and bovine eNOS genes reveals $75 \%$ sequence identity (Venema et al., 1994). Reporter gene assays using a fragment of the bovine promoter (positions -1548 to +240 ) in transfected BAEC showed a significant basal activity. A smaller fragment (-1548 to +192) which lacks two putative Sp-1 binding sites in the 5'-UTR, lost nearly all its promoter activity in the same system. This suggests that transcription factor $\mathrm{Sp}-1$ is important for bovine, as well as human eNOS transcription. Reports of an upregulation of eNOS mRNA in response to TGF- $\beta 1$ in BAEC are likely to be due to enhanced activity of the bovine promoter due to an increased binding of transcription factor NF-1 (Inoue et al., 1995).

\subsection{NOS Structure}

Nitric oxide synthases, like many other enzymes, have evolved to function in an oligomeric form. They are only active as dimers comprised of identical subunits. The homodimers are formed by two NOS subunits aligned in a head-to-head fashion, with the oxygenase domains of each subunit interacting functionally with the reductase domain of the companion subunit (see Fig.1.2). The structure of NOS monomers and dimers are discussed below.

Sequence analysis of all three NOS isoforms identified consensus binding sequences for reduced nicotinamide adenine dinucleotide phosphate (NADPH), flavin adenine dinucleotide (FAD), flavin mononucleotide (FMN) and CaM (Bredt et al., 1991; Lamas et al., 1992; Lyons et al., 1992; Xie et al., 1992), concurring with 


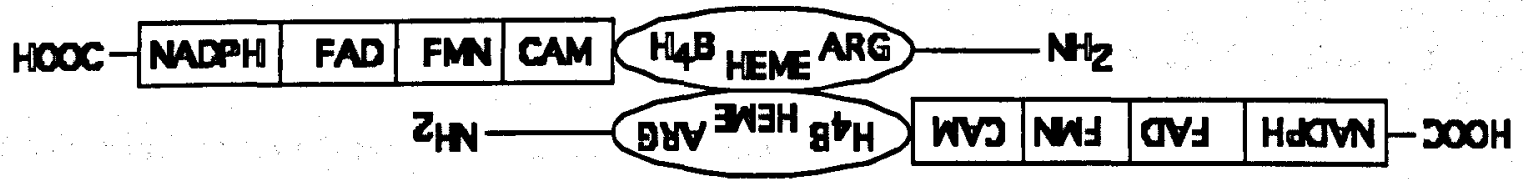

Fig 1.2 Formation of the NOS Dimer

The NOS dimeric structure is formed by a "head-to-head" association between the oxygenase domains of the two subunits with the reductase domains attached as independent extensions. 
previous data from purification studies that showed NOSs were NADPH dependent flavoproteins containing FMN and FAD, and capable of binding CaM. (Hevel et al., 1991; Mayer et al., 1991; Schmidt et al., 1992; Stuehr et al., 1991). The locality of these binding sites indicated a bidomain structure with a C-terminal reductase domain and a $\mathrm{N}$ terminal oxygenase domain, linked by a canonical $\mathrm{CaM}$ binding domain (see Fig.1.1).

The NOS reductase domain (C-terminal half) contains the NADPH, FAD and FMN binding domains and, taken on its own, displays $\approx 40 \%$ sequence homology to mammalian NADPH-cytochrome P450 reductase, the flavo-protein subunit of sulfite reductase and the reductase portion of the bacterial cytochrome P450 (Porter, 1991). As all of these enzymes transfer electrons from NADPH onto an endogenous or associated heme group; it was appreciated early that the role of the C-terminal region of NOS is to transfer electrons from NADPH onto a redox component in another region of the enzyme. The redox component in question has since been identified as iron protoporphyrin IX (heme) (Klatt et al., 1992; McMillan et al., 1992; Stuehr \& Ikeda-Saito, 1992; White \& Marletta, 1992) which was found to be bound within the N-terminal oxygenase domain of NOS.

Limited proteolysis studies of nNOS (Ghosh \& Stuehr, 1995; Sheta et al., 1994) and expression of individual domains (Chen et al., 1996; Gachhui et al., 1996; McMillan \& Masters, 1995; Salerno et al., 1996), allowed the positive assignment of the CaM-, flavin- and NADPH-binding sites to the reductase domain and the heme-, tetrahydrobiopterin- $\left(\mathrm{BH}_{4}\right)$, and $\mathrm{L}$-arginine-binding sites to the oxygenase domain. Point and deletion mutation studies of iNOS confirmed the involvement of residues 
1121-1144 in the reductase domain to be involved in NADPH binding (Xie et al., 1994). Peptides synthesized to correspond to the putative CaM binding domains have been shown to bind $\mathrm{CaM}$ with similar affinities to those demonstrated for the intact enzymes (Anagli et al., 1995; Persechini et al., 1996; Persechini et al., 1996; StevensTruss \& Marletta, 1995; Venema et al., 1996; Vorherr et al., 1993; Zoche et al., 1996). This confirms a role for the sequence in binding CaM. Nonetheless, studies using chimeric NOS constructs containing the CaM binding region of another isoform indicate that other areas of NOS may also contribute to CaM binding (Venema et al., 1996).

The heme iron group is axially coordinated in NOS by a Cys thiolate (Klatt $e t$ al., 1992; McMillan et al., 1992; Salerno et al., 1996; Stuehr \& Ikeda-Saito, 1992; Wang et al., 1993; White \& Marletta, 1992). Sequence and structural comparison of NOS with other thiolate-ligated hemoproteins such as cytochrome P450 have suggested that the coordinating Cys residue is located within the oxygenase domain at positions iNOS-194 (murine), nNOS-415 (rat) and eNOS-184 (bovine) (McMillan et al., 1992; Renaud et al., 1993). Point mutation studies involving these residues resulted in either the loss of heme binding (Chen et al., 1994; Richards et al., 1996; Xie et al., 1996) or the formation of an unstable heme containing species (McMillan \& Masters, 1995).

Specific $\mathrm{BH}_{4}$ or $\mathrm{L}$-arginine binding amino acids or regions within the oxygenase domain are poorly defined. Based on homology matching with other pterin-binding enzymes, three sites have been proposed, all are located C-terminally with respect to the heme-binding Cys residue. One of these sites can be discounted as 
it lies within the reductase domain and, as discussed earlier, $\mathrm{BH}_{4}$ has been shown to bind to the oxygenase domain (Chen et al., 1996; Gachhui et al., 1996; Ghosh \& Stuehr, 1995; McMillan \& Masters, 1995; Salerno et al., 1996; Sheta et al., 1994). A region in the oxygenase domain, iNOS residues $448-480$, was noted to be similar to the $\mathrm{BH}_{4}$ binding sites in the aromatic amino acid hydroxylases (Cho et al., 1995). Point mutations in this region of iNOS led to a loss of both $\mathrm{BH}_{4}$ binding and dimeric structure, but retained heme binding capability. Given this result, the role of dimer destabilization in the prevention of $\mathrm{BH}_{4}$ binding can not be ruled out, especially since it has been shown that monomeric iNOS and nNOS do not stably bind $\mathrm{BH}_{4}$ (Baek et al., 1993; Klatt et al., 1996). The third site (iNOS residues 340-494) shows some sequence homology to the dihydrofolate reductase pterin-binding domain (Nishimura et al., 1995), but studies using GST-fusion peptides corresponding to this region suggest that it cannot bind $\mathrm{BH}_{4}$. However such peptides were shown to be capable of binding radio-labeled nitroarginine, suggesting a role in L-arginine binding. Point mutagenesis of residues in the oxygenase domain $\mathrm{N}$-terminal to the heme-binding site (C99) resulted in NOS with decreased $\mathrm{BH}_{4}$ affinity, but normal characteristics for $\mathrm{L}$ arginine binding, activity and dimer formation. This suggested a possible role for C99 in modulating $\mathrm{BH}_{4}$ binding (Chen et al., 1994). Residues involved in $\mathrm{BH}_{4}$ and $\mathrm{L}$ arginine binding are present in the oxygenase domain and may be distributed on opposite, or possibly both sides of the heme binding domain.

The effects of arginine and $\mathrm{BH}_{4}$ on the oxygenase domain active site, and the heme-iron contained within, have been investigated using both the isolated oxygenase domain and the full length enzymes. It has been shown that the ferric heme-iron of NOS is predominately six-coordinate and low-spin in the absence of both $\mathrm{BH}_{4}$ and L- 
arginine (Fossetta et al., 1996; Martasek et al., 1996; Rodriguez-Crespo et al., 1996; Roman et al., 1995; Wu et al., 1996). Binding of $\mathrm{BH}_{4}$ and L-arginine favors a shift to a five-coordinate high-spin state, $\mathrm{BH}_{4}$ binding causes a mixture of the two spin states and arginine causing an almost complete shift to the high spin state. Binding studies using L-arginine or L-arginine analogues with $\mathrm{BH}_{4}$-containing ferric NOS, suggest that the binding of substrate can occur in several different modes with respect to the heme environment (Garvey et al., 1994; Salerno et al., 1995; Salerno et al., 1996). Inhibitor studies indicate that the NOS isoforms differ in the geometry of their substrate binding pockets (For review see Griffith \& Stuehr, 1995; Gross, 1995). It appears that $\mathrm{BH}_{4}$ and arginine both bind near the distal side of the heme group and can influence each other's binding, as well as the binding of heme-ligands, such as NO, CO, $\mathrm{CN}^{-}$and imidazole (Chabin et al., 1996; Wang et al., 1993). These effects were to be expected from L-arginine based on mechanistic considerations, but were surprising for $\mathrm{BH}_{4}$ and suggest a new role for this molecule in hemoproteins (discussed later in Mechanism section).

The oxygenase and reductase domains of iNOS or nNOS can be separated by trypsinolysis and purified by 5'-ADP sepharose chromatography (Ghosh \& Stuehr, 1995; Sheta et al., 1994). The individual domains seem to remain intact as they are unaltered in terms of prosthetic group binding and spectral properties. As predicted, neither domain alone was capable of NO formation from L-arginine. The oxygenase domains are homodimeric and reductase domains are monomeric. Hence, the oxygenase domain would seem to contain the determinants necessary for dimerization. This led to a head-to-head model for NOS dimerization with the oxygenase domains interacting to form the dimer and the reductase domains attached 
as independent extensions (Ghosh \& Stuehr, 1995). This model is supported by lightscattering studies which indicate that dimeric NOS has an elongated structure (Klatt et al., 1995) (see fig 1.2). However, immunoprecipitation studies using cells that coexpress eNOS and the eNOS reductase domain found that overexpression of the reductase domain prevented eNOS homodimer formation. Instead this seemed to cause the formation of a non-functional heterodimer comprised of one intact eNOS subunit and one eNOS reductase domain. Dimer dissociation studies show that the concentration of urea required to dissociate the oxygenase domain dimer is much higher than that required to cause the dissociation of the full-length iNOS dimer (Ghosh et al., 1996). This would suggest that the attached reductase domains destabilize the dimeric interaction between the two oxygenase domains. The role of the reductase domain in modulating dimeric interactions and active site structure is potentially important, but is as yet undefined..

The roles of the various prosthetic groups and substrates in promoting NOS dimerization have also been studied in both nNOS and iNOS. NOS monomers will not form dimers when incubated together at micromolar concentrations in a minimal buffer. However, it has been shown that dimerization of iNOS is promoted by the presence of L-arginine, $\mathrm{BH}_{4}$, and a stoichiometric amount of heme (Baek et al., 1993). The omission of $\mathrm{BH}_{4}$ reduced dimer association and the omission of heme or $\mathrm{L}$ arginine prevented dimer association. However, dimerization of heme-free nNOS monomers can be obtained in the presence of heme alone (Klatt et al., 1996), suggesting the role of heme insertion may be the most important factor in promoting dimer assembly in nNOS. The heme iron in the reconstituted iNOS dimer was ligated to the protein through a cysteine thiolate, and the dimer expressed a specific activity 
similar to the native dimeric iNOS (Baek et al., 1993). This would indicate that the prosthetic groups are bound properly in the monomers during dimerization. This data may suggest a role for heme and possibly $\mathrm{BH}_{4}$ and L-arginine in regulating NOS dimeric structure. Substrate or prosthetic groups affecting enzyme subunit assembly is not uncommon (for review see Jaenicke, 1996). However, the possible involvement of two prosthetic groups, heme and $\mathrm{BH}_{4}$, and substrate, L-arginine, is unusual and suggests multiple means by which NOS dimer assembly can be regulated in cells.

\subsection{NOS Mechanism and Possible Roles for Cofactors and Prosthetic Groups}

All three NOS isoforms catalyze a five-electron oxidation of one of the equivalent guanido nitrogens of L-arginine to yield one mole each of nitric oxide and L-citrulline, at the cost of 1.5 moles of NADPH and 2 moles of dioxygen (see Fig.1.3)(for review see Stuehr \& Griffith, 1992). The reaction involves two successive monooxygenation reactions with $\mathrm{N}^{\omega}$-hydroxy-L-arginine produced as an isolatable intermediate (Stuehr \& Ikeda-Saito, 1992). Isotopic studies have shown that NOS incorporates atoms from molecular oxygen into both citrulline and NO (Kwon et al., 1990; Leone et al., 1991). All NOS isoforms contain four prosthetic groups: FAD, FMN, Iron protoporphyrin IX (heme) and $\mathrm{BH}_{4}$. The flavins are involved in electron storage and delivery, accepting two electrons from NADPH and then delivering single electrons to the heme group within the active site (Bredt et al., 1991). The heme has an established redox role in the activation of molecular oxygen (McMillan et al., 1992; Stuehr \& Ikeda-Saito, 1992; White \& Marletta, 1992). Although essential to NO production, the role for $\mathrm{BH}_{4}$ is presently unclear. Based on information that the

stoichiometry of NADPH utilization is 1.5 NADPH per NO formed from arginine and 
0.5 NADPH per NO formed from NHA (Stuehr et al., 1991), several possible mechanisms have been proposed for NO production by NOS (Feldman et al., 1993; Klatt et al., 1993; Pufahl \& Marletta, 1993). In order to simplify these possible mechanisms it is first necessary to consider the roles of the individual co-factors.

\subsubsection{NADPH and Oxygen}

In the $\mathrm{N}$-oxidation of L-arginine, NADPH acts as a source of electrons for oxygen reduction-activation, as it does in cytochrome P450 (Watanabe et al., 1991). The overall reaction for cytochrome $\mathrm{P} 450$ can be written as follows:

\section{$\mathrm{O}_{2}+\mathrm{NADPH}+\mathrm{RH}+2 \mathrm{H}^{+}->\mathrm{H}_{2} \mathrm{O}+\mathrm{NADP}^{+}+\mathrm{ROH}$}

One atom of oxygen is incorporated into the substrate $(\mathrm{RH})$ and the other atom is fully reduced to water. The initial N-hydroxylation of arginine by NOS to give NHA is likely to occur by the same mechanism as depicted for cytochrome P450. However, subsequent NOS catalyzed transformation of the NHA intermediate to give citrulline and NO cannot be reconciled by cytochrome $\mathrm{P} 450$ mechanism as NO production constitutes an overall odd-electron oxidation. Consistent with this odd-electron oxidation, the conversion of NHA to NO has been found to require $0.5 \mathrm{NADPH}$ per NO formed (Stuehr et al., 1991).

\subsubsection{FAD and FMN}

There are two conceivable roles for the flavins present in NOS. On the one hand they have potential to act as the ultimate oxygen activators, forming 4a-hydroperoxyflavin and participating directly in the oxidation of the substrate, as they do in the 
monooxygenases (Ziegler, 1988). The alternative, much more likely role is that they act as an electron

buffer and transfer system. In this they would act as a link to transfer electrons from the NADPH onto the heme group, and eventually onto the substrate (see Fig.1.4). The high-degree of sequence homology between the flavin binding domains of NOS and cytochrome P450 reductase (Bredt et al., 1991), would suggest that the roles of the flavins in these two systems are identical. The cytochrome P450 system consists of two closely related enzymes, the flavin containing cytochrome $\mathrm{P} 450$ reductase and the heme containing cytochrome P450. In this system the role of the reductase is to bind NADPH and to transfer the electrons, via FAD and FMN, to the heme of the cytochrome P450. A similar role for the flavins in NOS is supported by the facts that both cytochrome $\mathrm{P} 450$ reductase and NOS are capable of cytochrome $\mathrm{c}$ reduction (Klatt et al., 1992; Mayer et al., 1992). Also, spectroscopic studies have indicated that the redox-active flavins in NOS are capable of interaction with the heme group (Stuehr \& Ikeda-Saito, 1992) and that a role of calmodulin is to enable this electron flux to occur (Abu-Soud \& Stuehr, 1993). Hence it is clear that the role of the flavins in NOS is to transfer the electrons from NADPH to the heme group for the eventual activation of molecular oxygen.

\subsubsection{Heme Group}

Based on the analogy between NOS and cytochrome P450 systems discussed above, it is clear the role for the heme in NOS is likely to be to bind, reduce and activate molecular oxygen to generate the ultimate oxidant. Also the heme environment in NOS appears to be similar to that of cytochrome P450 in that the heme is ligated axially by a thiolate (Stuehr \& Ikeda-Saito, 1992). Studies showing that carbon 
monoxide (CO) and other heme-binding agents inhibit arginine oxidation by NOS indicate a crucial catalytic role for the heme group (Klatt et al., 1992; McMillan et al., 1992; Stuehr \& Ikeda-Saito, 1992; White \& Marletta, 1992). A role for heme has also been established in the oxidation of the biosynthetic intermediate NHA (Pufahl \& Marletta, 1993). Hence the heme appears to be involved in the entire, multi-step oxidation pathway.

Both NOS and cytochrome P450 are known to 'uncouple' from substrate oxidation to generate reactive oxygen species such as superoxide and hydrogen peroxide (Heinzel et al., 1992; Mayer et al., 1992; Pou et al., 1992). In NOS isolated from pig brain, the degree of uncoupling appears to be increased at low concentrations of arginine (Heinzel et al., 1992). The production of superoxide or hydrogen peroxide would appear to occur via a heme-bound dioxygen intermediate.

\subsubsection{Tetrahydrobiopterin $\left(\mathrm{BH}_{4}\right)$}

The requirement of $\mathrm{BH}_{4}$ for maximal NOS activity in all NOS isoforms is well established (Hevel \& Marletta, 1992; Kwon et al., 1989; Mayer et al., 1991). However, of all the co-factors of NOS the role of $\mathrm{BH}_{4}$ is the least well understood and most controversial.

$\mathrm{BH}_{4}$ is known to be a co-factor in only four other enzyme systems, three of which are aromatic amino hydroxylases (phenylalanine, tyrosine and tryptophan hydroxylase). It is therefore not unreasonable to anticipate that $\mathrm{BH}_{4}$ would serve a similar role in NOS, an arginine hydroxylase (Kwon et al., 1989). Aromatic amino acid hydroxylases use $\mathrm{BH}_{4}$ directly for oxygenation of substrate via the formation of 


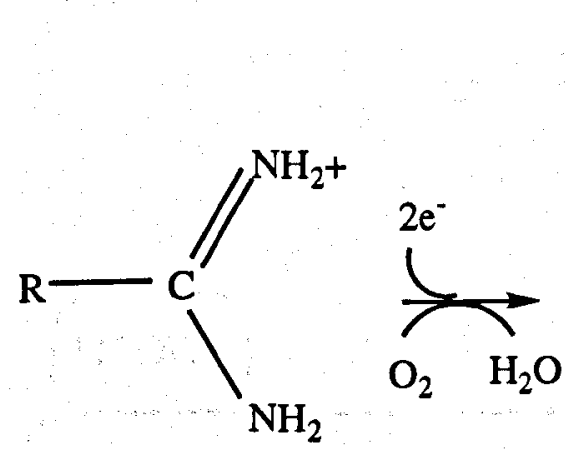

L-Arginine

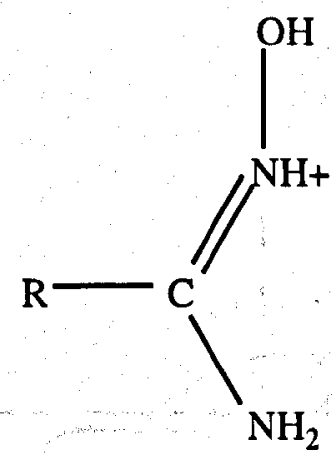

NG -Hydroxy-L-Arginine

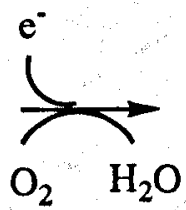

$\mathrm{R}$

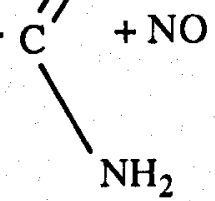

L-Citrulline $+$

Nitric Oxide

Fig. 1.3 Summary of the Nitric Oxide Synthase Catalyzed Reaction.

Electrons are donated by NADPH at both stages, with 1.5 moles of NADPH required for each mole of NO produced. 


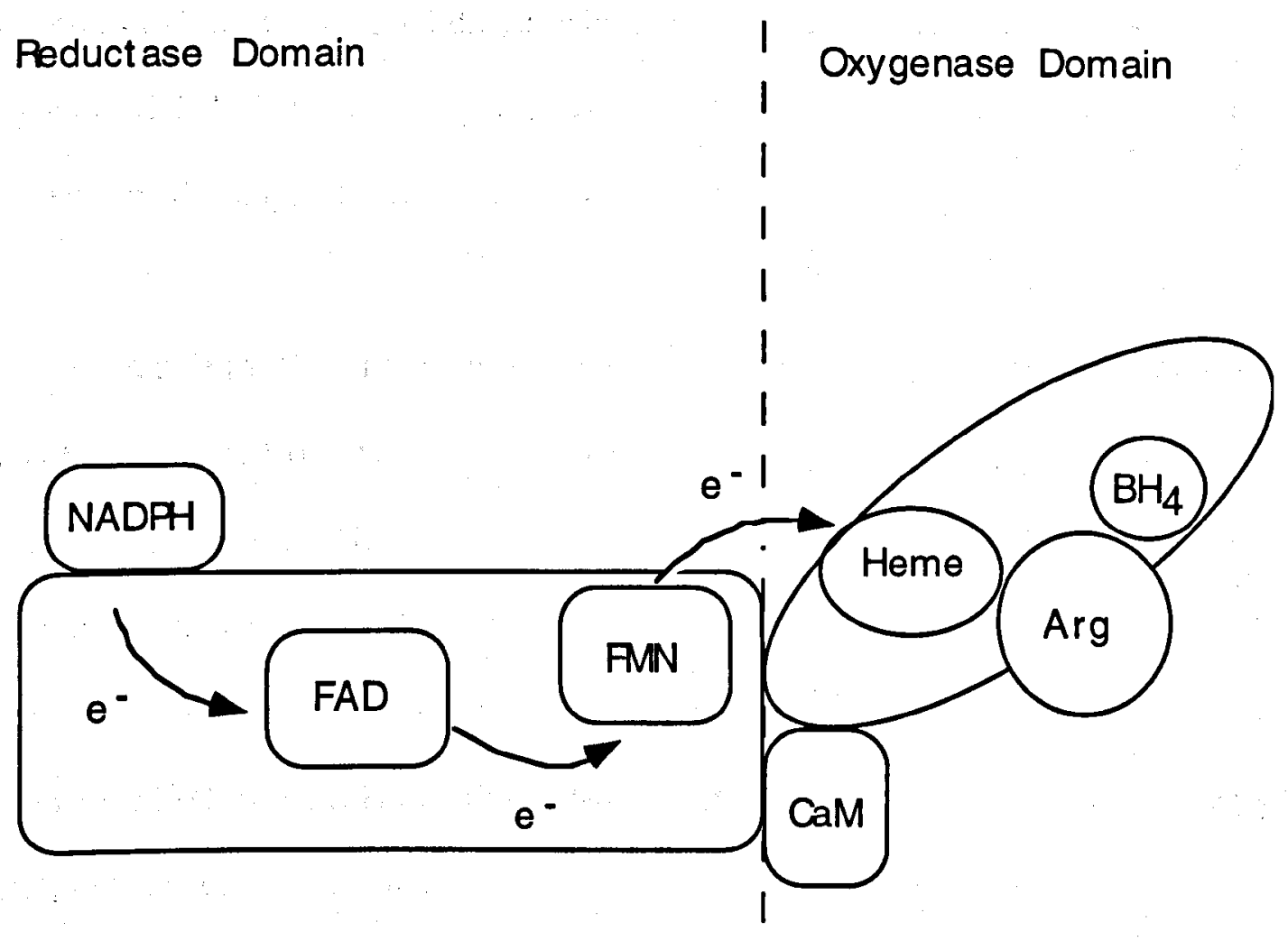

Fig. 1.4 Schematic Representation of Electron Flux Through NOS

While a monomer is shown for simplicity, electron transfer actually occurs between the reductase domain of one subunit and the oxygenase domain of the companion subunit of the homodimer. 
4a-hydroperoxybiopterin (Dix et al., 1987). After donating oxygen, this species becomes oxidized to quinoid dihydrobiopterin $\left(\mathrm{BH}_{2}-\mathrm{q}\right)$, which must then be recycled by an NADPH -dependent dihydropteridine reductase mediated reaction back to $\mathrm{BH}_{4}$ for sustained catalytic function (Nichol et al., 1985).

A role for $\mathrm{BH}_{4}$ participating as a direct redox-active agent in NOS catalysis has been rejected (Giovanelli et al., 1991), in favor of an allosteric or membrane stabilizing role for $\mathrm{BH}_{4}$. The main evidence against a direct redox role for $\mathrm{BH}_{4}$ stemmed from the fact that citrulline formation is not stoichiometric with $\mathrm{BH}_{4}$ consumption. Indeed for every $\mathrm{BH}_{4}$ consumed, more than 15 equivalents of citrulline are formed (Giovanelli et al., 1991). Isotopic studies have shown that the binding of $\mathrm{BH}_{4}$ induces conformational effects in NOS which can be detected as an increase in affinity for arginine-based ligands (Klatt et al., 1994; Liu \& Gross, 1996). Also spectroscopic and electron paramagnetic resonance studies have indicated that $\mathrm{BH}_{4}$ causes a low-spin to high-spin shift in the state of the NOS-bound heme group.

It has been established for rat nNOS that citrulline formation is not stoichiometric with $\mathrm{BH}_{4}, \mathrm{BH}_{4}$ has little effect on initial rate and $\mathrm{BH}_{4}$ is not recycled (Giovanelli et al., 1991). Hence it was suggested that $\mathrm{BH}_{4}$ act as an allosteric modulator or may serve a protective role by keeping a crucial catalytic component in the reduced form. 


\subsubsection{Overall Mechanism}

NOSs catalyze the five-electron oxidation of one of the chemically equivalent guanido nitrogens of L-arginine to form equimolar amounts of nitric oxide and Lcitrulline. This process requires oxygen as a co-substrate and NADPH as an electron source. The overall reaction has little or no chemical or biochemical precedent.

Due to the obvious similarities between NOS and cytochrome $\mathrm{P} 450$ reductase, it is likely that the two systems will share chemistry and enzymology in the catalysis of their respective reactions. However, there are two major differences which are immediately obvious. Firstly, cytochrome P450 enzymes typically perform a two (even) electron oxidation of its substrate whereas NOS catalyses a five (odd) electron step to produce NO. Secondly, cytochrome P450 does not share a requirement for $\mathrm{BH}_{4}$ with NOS. Because of these key differences, it is unlikely that the cytochrome P450 cycle can be directly applied to all the steps of NO production by NOS.

The first step in arginine oxidation appears to be N-hydroxylation of a terminal guanido nitrogen of L-arginine to give an N-hydroxy-L-arginine (NHA) intermediate. This is as simple two-electron oxidation of nitrogen and could be similar to other P450-catalyzed oxidations of nitrogen. The mechanism proposed for the nitrogen oxidation catalyzed by cytochrome P450 occurs via an initial electron transfer from nitrogen to the iron-bound oxygen followed by deprotonation (Macdonald et al., 1989; Miwa et al., 1983). Recombination of the subsequent radicals then results in a $\mathrm{N}$-hydroxylated species. 
This first step in NO production, N-hydroxylation of a guanidium nitrogen, is not unusual in that $\mathrm{N}$-oxidation is a common step in the biotransformation of many organic nitrogen compounds. However, further oxidation of the NHA or related compounds has little biochemical precedence. It has been proposed that a related compound containing an aromatic aminodoxime functionality could be oxidized by cytochrome P450 to give NO (Andronik-Lion et al., 1992). This represents a novel cytochrome $\mathrm{P} 450$ catalyzed reaction in that an odd electron product, NO, is the proposed product. Also peroxidation of NHA by cytochrome P450 and horseradish peroxidase to give citrulline has been proposed (Boucher et al., 1992). The chemical oxidation of $\mathrm{N}$-hydroxy-guanidines by a variety of mild oxidizing agents has shown that $\mathrm{HNO}$ and not NO is the preferred nitrogen oxide released under those oxidizing conditions (Fukuto et al., 1993). This is not surprising since HNO would be the expected two-electron oxidation product from a $\mathrm{N}$-hydroxyguanidine. It is therefore possible that NOS catalyzes a reaction in which the four electron oxidation of arginine gives rise to HNO, a process which would be entirely consistent with proposed cytochrome P450 chemistry. HNO could then be converted to NO by a one-electron oxidation, this pathway has been proposed and remains a viable mechanistic possibility (Stuehr \& Griffith, 1992). HNO has been shown to possess similar biological activity to that demonstrated by NO (Fukuto et al., 1992) and this similarity may be due to the conversion of HNO to NO under these oxidizing conditions (Fukuto et al., 1993).

Other mechanistic possibilities for NO generation from NHA all require a oneelectron oxidation step at some point in the catalysis. The donation of an electron from the substrate to the enzyme would account for the observed NADPH 
stoichiometry of $0.5 \mathrm{NADPH}$ (one electron equivalent) per NO formed. It has been proposed that NHA acts as a one electron reducing agent for the enzyme and subsequent reactions occur in a similar manner to those performed by cytochrome P450 aromatase (Marletta, 1993). A mechanism has also been proposed in which enzyme reduction by the NHA intermediate occurs prior to further oxidation via an iron-oxo species (Klatt et al., 1993).

\subsection{Regulation of NOS Activity}

Because of the lability and potential toxicity of NO, the activity of the NOSs must be tightly regulated to ensure correct physiological functioning. The three isoforms are regulated in different ways. Once synthesized, iNOS is constantly active and is regulated by substrate/co-factor availability and protein degradation. The regulation of cNOSs is more complex with regulation by intermittent binding to $\mathrm{Ca}^{2+} / \mathrm{CaM}$ and various other proteins.

\subsubsection{Trancriptional Regulation of iNOS}

iNOS is mainly regulated at the transcriptional level (see Fig. 1.5) and can be detected following cytokine or bacterial lipopolysaccharide (LPS) stimulation (Nathan \& Xie, 1994). Although, there is increasing evidence for "constitutive" expression of iNOS in some cell types (airway and renal epithelium, microvascular endothelium, and cell lines including PC-12, T84, AM) (Chu et al., 1995; Guo et al., 1995). 


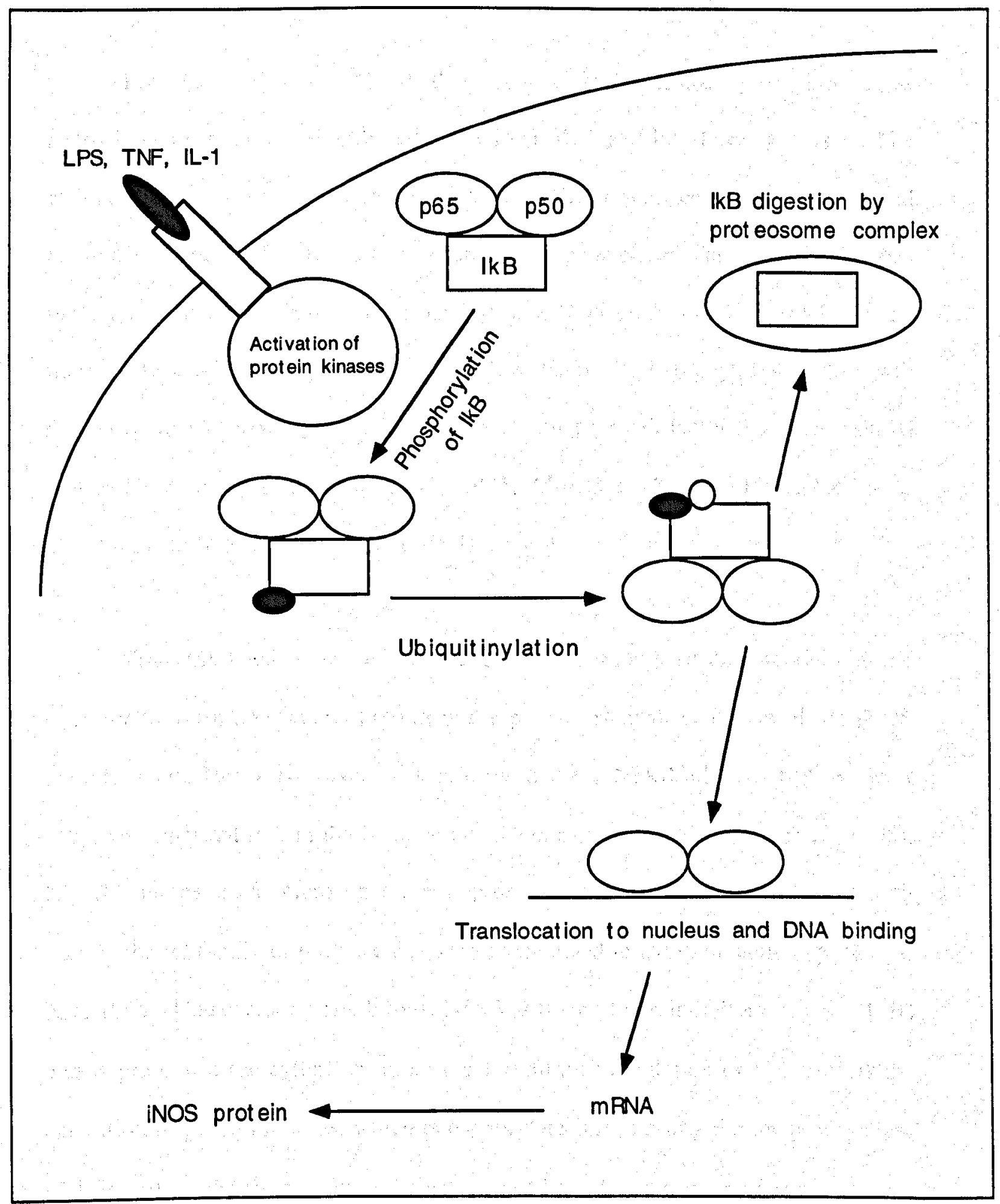

Fig 1.5 Schematic Representation of iNOS Induction. Stimuli bind to receptors at cell surface that initiate a signaling cascade, which leads to activation of $I \kappa B$ kinases and phosphorylation of IKB. Phosphorylation triggers the ubiquitinylation of $I \kappa B$, tagging the protein for degradation via the proteosome complex. The free NFKB can now translocate to the nucleus and initiate transcription of gene products, including iNOS. 
Proinflammatory cytokines that increase iNOS transcription are tumor necrosis factor- $\alpha$ (TNF- $\alpha$ ), interleukin-1 (IL-1), IL-2 and interferon- $\gamma$ (IFN- $\gamma$ ). The efficacy and potency of the various cytokines differ with cell type studied, and combinations of cytokines/LPS often exert a synergistic effect. There are also various molecules which can either increase or decrease iNOS mRNA and/or protein levels; these include inhibitory cytokines (IL-4, IL-8, IL-10, IL-13), peptides, and growth factors (platelet-derived growth factor, transforming growth factor- $\beta$, angiotensin II) (for review see Forstermann et al., 1995; Morris \& Billiar, 1994; Szabo \& Thiemermann, 1995; Xie \& Nathan, 1994).

Transcriptional control of iNOS is complex with at least 22 potential binding sites for transcription factors present in the promoter/enhancer region of the iNOS gene (Nathan, 1995). However, it is evident that the NFKB/rel transcription factor plays an important role in the induction of iNOS expression (Goldring et al., 1995). NFKB proteins are a heterogeneous group of proteins, and heterodimers containing mainly the rel family of proteins appear to be involved in iNOS induction (Nathan \& Xie, 1994). Under resting conditions, NFKB is bound to an inhibitory protein (IKB) and is present in the cytoplasm in an inactive form. Stimulation of cells results the activation of protein kinases, which phosphorylate $I \mathrm{~KB}$, causing the ubiquitinylation of IKB and results in the protein being "tagged" for degradation by the proteosome complex. NFKB is then free to translocate to the nucleus, where it can initiate gene transcription (Chen et al, 1995). The activation of NFKB often relies on the production of reactive oxygen species (Kroncke et al., 1995), although, exogenous NO has been shown to inhibit LPS/TNF-elicited NFKB activation (Colasanti et al, 1995). NO can also inhibit TNF synthesis in macrophages (Eigler et al., 1995). Hence 
there are feedback mechanisms whereby high levels of NO inhibit the induction of iNOS.

Expression of maximally active iNOS requires an efficient arginine transport system and the availability of cofactors such as $\mathrm{BH}_{4}$ (Morris \& Billiar, 1994). Most of the proteins responsible for the supply of these substrates/co-factors are co-induced with iNOS following cytokine activation of cells. Interference with the induction of the $\mathrm{Y}+$ transporter (responsible for arginine transport into the cell) or GTPcyclohydrolase I (GTPCH I, the rate limiting enzyme in the synthesis of $\mathrm{BH}_{4}$ ), would decrease NO production in cells. The intricacies of iNOS regulation are not fully understood and further work in this area is required.

\subsubsection{Regulation of NOS Activity by Calmodulin}

Although the CaM-dependent regulation of enzymes is very common in physiology, the NOSs are the first enzymes in which CaM has been shown to regulate electron flux (Abu-Soud \& Stuehr, 1993). This is assumed to be due to the ability of $\mathrm{CaM}$ to align the reductase flavin groups with the oxygenase heme groups, leading to efficient inter-domain electron transfer. However, $\mathrm{CaM}$ has also been shown to promote inter-flavin electron flux (Abu-Soud et al., 1994), an effect which involves the reductase domains only. Conceivably, the rate-limiting step in electron transfer within NOSs (at least eNOS,) (Nishida \& Ortiz de Montellano, 1998) is actually within the reductase domain and the principal or sole effects of $\mathrm{CaM}$ may be at this site. Future high resolution crystal structures of CaM-bound and CaM-free cNOS will be needed to reconcile these possibilities. 
Despite the void in direct structural information, a prototypic CaM binding site composed of basic and hydrophobic amino acids was clearly recognized in the deduced amino acid sequence of the first NOS cDNA to be cloned (residues 725-745 of rat nNOS) (Bredt et al., 1991). Subsequent studies of CaM binding to synthetic peptides confirmed this identification in nNOS (Vorherr et al., 1993; Zhang et al., 1995) and the corresponding amino acid sequences in the other NOS isoforms (Anagli et al., 1995; Venema et al., 1996). CaM binding by the nNOS-derived peptide was demonstrated to have an estimated $\mathrm{K}_{\mathrm{d}}$ of $2 \mathrm{nM}$ (Vorherr et al., 1993). Notably, the CaM-bound peptide adopted an $\alpha$-helical conformation (Zhang \& Vogel, 1994), a structure which was not stable in the absence of bound CaM. NMR studies showed that this complex has structural features in common with other complexes of $\mathrm{Ca}^{2+} / \mathrm{CaM}$ and target peptides such as that from myosin light chain kinase (Zhang et al., 1995). The nNOS peptide binds with a $\mathrm{C}$ to $\mathrm{N}$ orientation to the $\mathrm{N}$ - and $\mathrm{C}$-terminal domains of $\mathrm{CaM}$ and interaction appears to involve methionine-rich hydrophobic patches, similar to the interactions of $\mathrm{CaM}$ with other well-studied CaM-binding peptides (Zhang et al., 1995). Tryptophan fluorescence quenching studies using nNOS holoenzyme indicate a similar $\mathrm{K}_{\mathrm{d}}(1 \mathrm{nM})$ for CaM binding (Sheta et al., 1994). In the absence of bound $\mathrm{CaM}$, the $\mathrm{N}$-terminal peptide bond of arginine 728 in the CaM-binding sequence of bovine nNOS was shown to be hypersensitive to trypsinolysis (Lowe et al., 1996) and CaM binding prevented trypsinolysis at this site. Combined with other findings, these results suggested that in the absence of $\mathrm{Ca}^{2+} / \mathrm{CaM}$, the CaM binding site is exposed on the surface of nNOS and is rather flexible, but adopts a more rigid structure when complexed with $\mathrm{Ca}^{2+} / \mathrm{CaM}$. 
The precise molecular interactions of $\mathrm{CaM}$ required for activation of the cNOSs has not been elucidated, however, investigations using mutant CaM proteins have provided some insights into this process. $\mathrm{CaM}$ is a $17 \mathrm{KD}$ protein composed of an 8 -turn central $\alpha$-helix, flanked by globular $\mathrm{N}$-terminal and $\mathrm{C}$-terminal $\mathrm{Ca}^{2+}$-binding lobes (Babu et al., 1988). Each lobe consists of two EF-hand motifs that possess the capacity for binding a single $\mathrm{Ca}^{2+}$ atom (Persechini et al., 1989). Binding of $\mathrm{Ca}^{2+}$ causes a conformational change, exposing hydrophobic and acidic residues which engage in binding to the relevant regulatory sites on target proteins (Weinstein \& Mehler, 1994). Using engineered CaM proteins expressing two C- or two $\mathrm{N}$-terminal lobes, each of these lobes have been shown to bind to nNOSs independently (Persechini et al., 1994). However, while binding of C-C CaM to nNOS elicited no activation, binding of $\mathrm{N}-\mathrm{N} \mathrm{CaM}$ allowed full activation of the enzyme (Persechini $e t$ al., 1996). Hence, binding of the N-terminal lobe of CaM is required for nNOS activation.

Whether or not a given NOS isoform binds $\mathrm{CaM}$ in a $\mathrm{Ca}^{2+}$-dependent (cNOSs) or $\mathrm{Ca}^{2+}$-independent manner (iNOS) was assumed to be solely a property of the 20-25 amino acid sequence that comprises the CaM binding site. Thus, it was unexpected when eNOS and nNOS chimera, engineered to have their native CaM binding sites substituted with a homologous sequence from murine iNOS, were demonstrated to retain a $\mathrm{Ca}^{2+}$-requirement for full activity (Ruan et al., 1996; Venema et al., 1996). Studies of NOS chimera suggested the CaM binding site, on its own, is insufficient to explain the observed $\mathrm{Ca}^{2+}$-dependence of NOS isoforms. Hence, there must be other, as yet unknown, regions within the sequence of the NOSs, that contribute to the observed $\mathrm{Ca}^{2+}$-dependence of the NOS isoforms. 


\subsubsection{Intracellular Localization, Protein-Protein Interactions and Posttranslational}

\section{Modification of NOS Isoforms}

Targeting to specific subcellular regions is an important mechanism for the regulation of various signaling proteins, including protein kinases, phosphatases, and G-proteins. This process of targeting is often under the control of posttranslational modifications (phosphorylation, acylation) and interactions between the targeted protein and structural proteins in the designated subcellular region (Blenis \& Resh, 1993; Resh, 1994; Resh, 1996).

Phosphorylation is an important mechanism for the posttranslational regulation of several key proteins, including hormone receptors, transcription factors and several intracellular effector molecules (Krebs \& Beavo, 1979). Phosphorylation of NOSs is of particular interest as it would allow cross talk between several different signaling pathways. Sequence analyses of NOSs reveal several consensus sequences for phosphorylation, including putative sites for cyclic AMP-dependent protein kinase (PKA), protein kinase $\mathrm{C}(\mathrm{PKC})$, cyclic GMP-dependent protein kinase $(\mathrm{PKG})$, protein kinase B (PKB/AKT), casein kinase II and calmodulin-dependent kinase II (CaMKII). However, the functional significance of these sites has not been established in most cases. Earlier reported studies have been inconclusive and, in some cases, contradictory. However, great strides in the understanding of phosphorylationmediated regulation of eNOS in particular, has been made during the last year. 
Due to the labile nature of NO and its important role in signaling, discrete subcellular localization of NOS proteins within the cell might be expected. Indeed, all three NOS isoforms have been shown to be localized to distinct subcellular regions, undergo posttranslational modifications and to interact with various cellular proteins. The regulation and functional significance of these processes is discussed in the following section.

\subsubsection{1 iNOS}

\subsection{Subcellular Localization}

iNOS was originally purified as a soluble cytosolic protein (Stuehr et al., 1991), it has since become apparent that this enzyme may undergo tissue specific targeting to distinct subcellular organelles, although the evidence is somewhat limited. Inducible NOS can be found in the particulate subcellular fraction of freshly isolated tissue macrophages (Stuehr, 1997; Vodovotz et al., 1995), to a novel subcellular organelle that has been termed the "nitroxysome" (Vodovotz et al., 1995). The nitroxysome may serve to facilitate the NO-dependent killing of opsonized intracellular microorganisms. Nonetheless, factors which determine cytosolic vs. subcellular targeting of iNOS remain to be established.

\subsection{Protein-Protein Interactions}

Until recently it was thought that iNOS activity was regulated purely by protein degradation and substrate/co-factor availability. However, recent evidence points to the existence of an iNOS-associated regulatory protein. Murine macrophages were shown to express a $110 \mathrm{kDa}$ protein (NAP 110), which directly interacts with the amino terminus of iNOS and inhibits catalytic activity by preventing the formation of 
NOS homodimers (Ratovitski et al., 1999). The expression of this protein may be a mechanism by which macrophages protect themselves from the production of toxic levels of nitric oxide. Additionally, a proposed interaction between iNOS and the Rho family GTPase, Rac2 (Kone \& Kuncewicz, 1998) has been suggested. This interaction was verified by co-immunoprecipitation of iNOS and Rac2 from lipopolysaccharide treated macrophages. Overexpression of Rac2 in RAW 264.7 macrophage cells resulted in increased NO generation and suggested that Rac2 serves as an allosteric activator of iNOS.

\subsection{Posttranslational Modifications}

Although phosphorylation of iNOS has been demonstrated in cell culture (Nathan \& Xie, 1994), the functional or cellular consequences have not yet been established. Overall the regulation of iNOS seems to occur mainly at the transcriptional level (See gene regulation section) and the evidence for posttranslational regulation is limited.

\subsubsection{2 nNOS}

\subsection{Subcellular Localization}

Like iNOS, nNOS was also originally purified as a soluble cytosolic protein (Bredt \& Snyder, 1990), it has since been demonstrated that this enzyme may undergo tissue specific targeting to distinct subcellular regions. The regulation of this process is discussed below.

Immunoelectron microscopy has demonstrated nNOS localization to the membrane regions of certain neuronal cells and colocalization with NMDA receptors (Brenman et al., 1996). A similar situation occurs in skeletal muscle where nNOS is also localized to the plasma membrane (Kobzik et al., 1994) and in macula densa 
cells in the rat kidney where nNOS was specifically localized to the basolateral membrane (Tojo et al., 1999).

\subsection{Protein-Protein Interactions}

The localization of nNOS seems to occur by protein-protein interactions associated with a specific region of the $\mathrm{N}$-terminal region of $\mathrm{nNOS}$. The $\mathrm{N}$-terminal sequence of nNOS is extended in relation to the other isoforms ( 300 amino acids) and it was noted that residues 18-97 (rat nNOS) contained a motif which is termed a "GLGF repeat" or "PDZ domain" (Hendriks, 1995). This motif is shared in common with proteins which localize to cell-cell junctions (Hendriks, 1995).

In the case of neuronal cells, nNOS associates with the post synaptic density (PSD) proteins, either PSD-95 or PSD-93, depending on location within the brain (Brenman et al., 1996; Brenman et al., 1996). These interactions occur via the PDZ domain-PDZ domain interactions. These PSDs associate with NMDA receptors and the complex of NMDA receptor-PSD-nNOS may ensure that $\mathrm{Ca}^{2+}$ influx through NMDA receptors is specifically coupled to nNOS activation. Another nNOSassociated protein, CAPON, has been identified in neural tissues and is thought to function by regulating the interactions of nNOS with PSD-95 (Jaffrey et al., 1998). In the kidney nNOS and PSD-93 were similarly found to co-localize to membrane regions of macula densa cells (Tojo et al., 1999).

nNOS associates via its PDZ motif with the membrane-bound dystrophin complex in skeletal muscle; this association is responsible for the localization of nNOS to the sarcolemmal membrane (Brenman et al., 1995). In this setting, it was 
demonstrated that nNOS specifically associates with another PDZ domain containing protein, $\alpha 1$-syntrophin, a component of the dystrophin complex (Brenman et al., 1996). This targeting seems to serve two purposes. Firstly, this localization may improve the efficiency of $\mathrm{Ca}^{2+}$ influx-mediated nNOS activation via calmodulin. Secondly, NO synthesis at the plasma membrane facilitates delivery of NO to surrounding cellular targets such as the vasculature (Bredt, 1998).

nNOS also seems to interact with another protein, protein inhibitor of nNOS (PIN), this 89 amino acid protein inhibits nNOS by destabilizing the active homodimer (Jaffrey \& Snyder, 1996). The PIN binding domain of nNOS (amino acids 161-245) is absent from the other NOS isoforms allowing for specific inhibition of nNOS (Jaffrey \& Snyder, 1996). The pattern of expression of PIN and nNOS in the different brain regions are nearly parallel, supporting the idea that PIN may function as an in vivo regulator of nNOS activity (Greenwood et al., 1997). A neuroprotective role for PIN is also supported by studies which show that PIN is induced in the brain following global ischaemia (Gillardon et al., 1998), defining regions that are resistant to ischaemic damage.

A protein interaction of nNOS which has recently come to light is a proposed inhibitory interaction between nNOS and the $4^{\text {th }}$ intracellular domain of the bradykinin B2 receptor (B2R) (Golser et al., 2000). In this study, synthetic peptides corresponding to the $4^{\text {th }}$ intracellular loop of this receptor were shown to form a complex with nNOS and inhibited NO formation with an $\mathrm{IC}_{50}$ of $\approx 7 \mu \mathrm{M}$. These studies also demonstrated the coimmunoprecipitation of B2R and nNOS from human 
embryonic kidney (HEK 293) cells that had been stably transfected with human $\mathrm{nNOS}$; results in an in vivo interaction between $\mathrm{nNOS}$ and $\mathrm{B} 2 \mathrm{R}$.

\subsection{Posttranslational Modification}

The Regulation of nNOS by phosphorylation is poorly understood. Several studies have demonstrated the phosphorylation of nNOS, although the effects upon enzyme activity are inconclusive. One laboratory has reported that PKA, CaM Kinase II and PKC, can stoichiometrically phosphorylate nNOS in vitro, but only the activation of PKC resulted in phosphorylation in vivo (Bredt et al., 1992). The in vitro phosphorylation did not modify NOS activity, whereas in vivo phosphorylation by PKC resulted in depression of nitrite production, despite constant levels of NOS protein. These results would indicate that agonists which activate phospholipase C and the phosphoinositide pathway could send signals that both activate and inactivate NOS. The IP3-mediated calcium release, which activates NOS and the activation of PKC by diacylglycerol leads to the phosphorylation and inactivation of NOS. This could serve as a possible regulatory mechanism. However, other in vitro studies have demonstrated that only PKA, not PKC or CamKII, phosphorylate nNOS and that this PKA-mediated phosphorylation had no effect on activity (Brune \& Lapetina, 1991). Studies by yet another group demonstrated phosphorylation by PKC and CaMKII with an associated increase or decreased in NOS activity, respectively (Nakane et al., 1991). This was partially contradicted by experiments in which both PKA and c-GMP dependent protein kinase (PKG) phosphorylated nNOS and diminished catalytic activity in vitro. Cell culture studies using HEK-293 cells stably transfected with nNOS, showed phosphorylation of nNOS and a reduction of nitrite release following activation of PKG by 8-Bromo-cGMP (Dinerman et al., 1994). It has even been 
suggested by one group that nNOS is capable of autophosphorylation, although the effect on activity was not studied (Watanabe et al., 1996) and no follow up studies have been published. The discrepancies in these studies indicate the obvious need to further investigate the extent to which phosphorylation of nNOS occurs, conditions that promote phosphorylation, the effect on activity, and the physiological relevance.

\subsubsection{3 eNOS}

\subsection{Subcellular Localization}

In contrast to the other two isoforms, the eNOS isoform was originally characterized as a membrane-associated protein, purified from a detergent solublized cell fraction (Pollock et al., 1991). However, it has since been observed that eNOS targeting to the plasma membrane may be dynamically modulated in a reversible manner, a process which makes eNOS unique amongst NOS isoforms.

eNOS is localized to specific regions of the plasma membrane termed caveolae (Shaul et al., 1996). These are small invaginations in the plasma membrane characterized by a distinctive lipid composition and by the presence of the transmembrane protein caveolin (For reviews see Anderson, 1998; Anderson, 1993; Schlegel et al., 1998). Caveolae are a prominent feature of the endothelial cell plasma membrane and are also present in cardiac myocytes, adipocytes, and numerous other cell types. These caveolae appear to be sites for sequestration of signaling molecules such as receptors, G-proteins, protein kinases, as well as eNOS. 


\subsection{Protein-Protein Interactions}

Subsequent to the original observation of the caveolar localization of eNOS (Shaul et al., 1996), it was reported that eNOS is quantitatively associated with caveolin in endothelial cells and cardiac myocytes (Feron et al., 1996). Further studies revealed that this association leads to the inhibition of enzyme activity, and that a proteinprotein interaction takes place between the two proteins (Feron et al., 1998; GarciaCardena et al., 1997; Ju et al., 1997; Michel et al., 1997; Michel et al., 1997). Consensus sequences were identified within both proteins: the scaffolding domain of caveolin, a juxtamembranous 20 amino acid sequence in the C-terminal region of caveolin (Ju et al., 1997; Michel et al., 1997) and the caveolin binding motif, a peptide sequence that is rich in aromatic residues in the oxygenase domain of eNOS (Feron et al., 1998; Garcia-Cardena et al., 1997). The caveolin binding motif is shared with other caveolae-associated proteins, including H-ras, G-protein- $\alpha$, the src family of proteins, mitogen activated protein kinase, epidermal growth factor receptor and protein kinase $\mathrm{C}$, all of which also form inhibitory complexes with caveolin (Okamoto T et al, 1998) (Shaul \& Anderson, 1998). However, eNOS also has motifs present in the reductase domain which associate with the N-terminal end of caveolin (Ghosh $e t$ al., 1998; Ju et al., 1997).

An interesting factor governing the eNOS-caveolin association, is that $\mathrm{CaM}$ disrupts the complex in a $\mathrm{Ca}^{2+}$-dependent fashion and completely reverses the inhibitory effect of caveolin (Michel et al., 1997). Caveolin serves as a competitive inhibitor of calmodulin-dependent eNOS activation (Michel et al., 1997). Hence, calmodulin binding to the CaM binding sequence of the eNOS reductase domain activates the enzyme by promoting inter-domain electron flux, and in the process 
displaces caveolin, relieving the inhibitory effects (Ghosh et al., 1998; Michel et al., 1997). This process has also been shown to occur in intact cells with dissociation of the eNOS-caveolin complex promoting translocation of eNOS from the caveolae (Feron et al., 1998).

Another protein which has been shown to directly interact with eNOS is heat shock protein 90 (HSP90) (Garcia-Cardena et al., 1998). This protein was originally termed eNOS-associated protein 1 (ENAP-1) (Venema et al., 1996) and was first detected in bradykinin treated endothelial cells. The interaction of eNOS with HSP90 facilitates eNOS activation in endothelial cells following stimulation with a $\mathrm{Ca}^{2+}$ mobilizing agonist or shear stress application (Garcia-Cardena et al., 1998) (See $\mathrm{Ca}^{2+}$ independent activation of eNOS Section). More recent evidence indicates that HSP-90 (or an associated protein) promotes the calmodulin-dependent displacement of eNOS from the eNOS-caveolin complex (Gratton et al., 2000).

The arginine transporter CAT-1 represents another protein with which eNOS has been shown to interact. Coimmunoprecipitation of eNOS and CAT-1 has been demonstrated and both are localized to the membrane caveolae (McDonald et al., 1997). This particular interaction may help to explain "the arginine paradox". Simply stated, this paradox is that half-saturating arginine concentration for $\mathrm{eNOS}$ is $<10 \mu \mathrm{M}$ (Liu et al., 1996; Palmer \& Moncada, 1989; Pollock et al., 1991), while intracellular endothelial arginine concentration is typically millimolar (Block et al., 1995; Hecker et al., 1990; Mitchell et al., 1990), yet NO production by endothelial cells is dependent upon an adequate and continuing supply of extracellular arginine (Aisaka et al., 1989; Cooke et al., 1991; Taylor \& Poston, 1994). At these levels of 
intracellular arginine, eNOS should be saturated and the addition of extracellular arginine should not cause an increase in NO synthesis. An association between the CAT-1 transporter and eNOS, facilitating a direct supply of exogenous arginine to eNOS provides explanation of this apparent paradox.

Other caveolar proteins have also been implicated in interactions with eNOS. The bradykinin B2 receptor (B2R) and the angiotensin II type I receptor $\left(\mathrm{AT}_{1}\right)$ have both demonstrated an ability to form an inhibitory complex with eNOS via the $4^{\text {th }}$ intracellular loop region (Golser et al., 2000; Ju et al., 1998). In the case of the B2ReNOS interaction, a degree of functionality has been demonstrated. In vitro binding and co-immunoprecipitation studies demonstrate that the B2R interacts with eNOS in a ligand- and $\mathrm{Ca}^{2+}$-dependent manner (Ju et al., 1998). In the resting state, the receptor docks with eNOS in the caveolar membrane and participates in its inactivation. Activation of endothelial cells by bradykinin or $\mathrm{Ca}^{2+}$ ionophore promotes the dissociation of the eNOS-B2R complex coincident with the activation of eNOS (Ju et al., 1998). The site of binding to eNOS and the mechanism by which the B2R exerts this inhibition seems to be distinct from those by which caveolin-l interacts with, and inhibits eNOS. A similar in vitro interaction has been demonstrated between eNOS and $\mathrm{AT}_{1}$-glutathione-s-transferase fusion protein (Ju et al., 1998), however, no stimulus-dependent dissociation of the complex was demonstrated. If physiological, this process of effector activation by agonist-induced receptor-effector complex dissociation, represents a novel mechanism of receptor-dependent effector activation. 


\subsubsection{Posttranslational Modification}

eNOS is regulated by a complex pattern of covalent modification, including phosphorylation (Michel et al., 1993), myristoylation (Busconi \& Michel, 1993; Liu \& Sessa, 1994; Pollock et al., 1992), and palmitoylation (Robinson et al., 1995; Robinson \& Michel, 1995). All of these covalent modifications have an impact on the subcellular targeting of eNOS, the effects of the individual modifications are considered below.

\subsection{Phosphorylation}

The phosphorylation of eNOS has been demonstrated in several studies and was, until recently, a very controversial area of eNOS regulation. Older studies using cell culture of metabolically ${ }^{32} \mathrm{P}$-labelled bovine arterial endothelial cells (BAECs) showed a several fold increase in basal phosphorylation of eNOS in response to bradykinin, ionomycin and the NO donor, sodium nitroprusside (Michel et al., 1993). The increase was time dependent and not associated with an increase in de novo synthesis of NOS protein. The bradykinin induced phosphorylation was inhibited by the calmodulin antagonist, H-7, but was unaffected by inhibitors of PKA, PKC, CaMKII or myosin light chain kinase (MLCK). This suggested that either the phosphorylation was dependent on the activation of eNOS or the kinase responsible was calmodulin-dependent (but not CaMKII). Phosphorylation of eNOS was also found to be associated with the translocation of the enzyme from the membrane bound to the cytosolic compartment. Under normal conditions, $90-95 \%$ of eNOS is located in the particulate fraction of endothelial cells. Upon stimulation with bradykinin, approximately $50 \%$ of total ${ }^{35} \mathrm{~S}$-methionine labeled enzyme and $85 \%$ of 
the total phosphorylated enzyme was cytosolic. Both the phosphorylation and translocation are blocked by $\mathrm{H}-7$.

Another stimulus that has been demonstrated to cause activation and phosphorylation of eNOS is fluid shear-stress; this is caused by increases in blood flow through a blood vessel (Fleming et al., 1997). In the case of activation by fluid shear-stress, there is also some evidence for intracellular mechanisms which are able to influence eNOS activity at basal intracellular calcium levels. In studies using cultured endothelial cells, a disparity is apparent in the temporal relationship between the duration of the agonist-induced increases in intracellular calcium and the duration of activity of eNOS. The duration of the calcium response being significantly shorter than the duration of the subsequent period of eNOS activity (Fleming et al.; 1997; Fleming et al., 1994). Studies suggest that this $\mathrm{Ca}^{2+}$-independent pathway for activation of eNOS is abrogated by tyrosine kinase inhibitors, such as erbstatin $\mathrm{A}$ (Ayajiki et al., 1996; Fleming et al., 1997).

However, the lack of an identified eNOS kinase for either shear-stress or mitogenic stimuli was problematic in the interpretation of these results. Recent studies by two independent groups cleared up much of the confusion surrounding the regulation of eNOS by phosphorylation with their identification of at least one of the kinases involved (Dimmeler et al., 1999; Fulton et al., 1999). The relevant kinase turned out to be the recently cloned protein kinase B (Masure et al., 1999), also known as Akt. This kinase is activated via the phosphatidylinositol 3-kinase (PI-3K) pathway, resulting in the production of membrane-restricted second messenger molecules (polyphosphatidylinositides containing a 3'-phosphate) (for review see 
Coffer et al., 1998; Downward, 1998). Inactive Akt complexes interact with these 3phosphorylated inositides via a pleckstrin homology domain. Once targeted to the membrane via this motif, Akt becomes phosphorylated at two residues which relieves the intermolecular inhibition, allowing the activated complex to dissociate and modify its targets, eNOS included. In these studies, it was demonstrated that phosphorylation of serine 1179 (bovine sequence, 1177 in the human sequence) is involved in the activation of eNOS in response to stimuli including shear-stress and vascular endothelial growth factor (VEGF). Furthermore, transfection of a constitutively active mutant of Akt increased basal NO release from cultured endothelial cells, conversely transfection of an activation-deficient Akt attenuated the NO response to VEGF. Serine 1179 was confirmed as the site of phosphorylation by the transfection of a serine 1179 phosphorylation mimic (Aspartate 1179) or a mutant incapable of phosphorylation of this residue (Alanine 1179) which produced constitutive activity or an inability to be activated by the normal stimuli, respectively. Phosphorylation of this residue has since been shown to be required for the activation of eNOS in response to traditionally $\mathrm{Ca}^{2+}$-independent stimuli, such as shear-stress, as well as $\mathrm{Ca}^{2+}$-dependent stimuli, such as insulin-like growth factor (Michell et al., 1999), acetylcholine (Luo et al., 2000) and VEGF (Dimmeler et al., 2000; Fulton et al., 1999; Michell et al., 1999). Additionally, mass spectrometry identified this site as the predominant site of phosphorylation in eNOS in response to shear-stress and that LY294002, an inhibitor of phosphatidylinositol kinase, abrogated this phosphorylation by $97 \%$ and NO production by $68 \%$ (Gallis et al., 1999). Interestingly, in cultured human cardiac myocytes, this site has been shown to be a target for phosphorylation by PKA (Chen et al., 1999). In vitro studies of a mutant eNOS mimicking phosphorylation of this site (aspartate 1179) showed a 2-4 fold 
increase in the rates of NO production and cytochrome $\mathrm{c}$ reduction. This mutant also exhibited an increased resistance to inactivation by $\mathrm{Ca}^{2+}$-chelation. These results suggest that phosphorylation of this residue increases catalytic activity by increasing the rate of electron flux through the reductase domain and attenuating $\mathrm{CaM}$ dissociation from eNOS at sub-optimal levels of free $\mathrm{Ca}^{2+}$ (McCabe et al., 2000).

\subsection{Acylation}

As mentioned earlier, eNOS undergoes another form of posttranslational regulation, acylation, a characteristic which makes it unique amongst NOS isoforms. eNOS undergoes both myristoylation (Busconi \& Michel, 1993; Liu \& Sessa, 1994; Pollock et al., 1992), and palmitoylation (Robinson et al., 1995; Robinson \& Michel, 1995). Both modifications occur at the N-terminus of the enzyme with palmitoylation occurring at glycine 2 (Busconi \& Michel, 1993; Sessa et al., 1993) and palmitoylation at cysteine residues 15 and 26 (Garcia-Cardena et al., 1996; Liu et al., 1995). The exact contribution of each of these modifications to eNOS regulation is unclear at the present.

From mutation studies it is known that myristoylation of eNOS is necessary for targeting the enzyme to the endothelial plasma membrane (Busconi \& Michel, 1993). However, this cannot control the reversible translocation of eNOS between membrane and cytosol, as myristoylation is typically cotranslational and irreversible, precluding its dynamic regulation by agonists (Resh, 1994). Also, for other myristoylated proteins, additional hydrophobic or electrostatic interactions (in addition to those between myristate and membrane lipids) are required for stable membrane association. Hence, reversible posttranslational modifications, such as 
phosphorylation or palmitoylation, often regulate the subcellular localization of myristoylated proteins.

As discussed earlier, it has been shown that eNOS undergoes phosphorylation, which is enhanced by agonists that also promote migration of the protein from the membrane to the cytosolic fraction (Michel et al., 1993). This raises the possibility that phosphorylation of eNOS at the cell membrane triggers its translocation to the cytosol. However, a mutant eNOS lacking the myristoylation site, still undergoes phosphorylation despite being restricted to the cell cytosol, precluding this as the cause of translocation.

It was subsequently discovered that eNOS underwent reversible palmitoylation (Robinson et al., 1995). Due to the reversible nature of this acylation (Resh, 1994), it provides a potential mechanism for the regulation of agonist induced changes in the subcellular localization of eNOS and agonist induced changes in palmitoylation status have previously been described for G-protein-coupled receptors, such as the $\beta$-adrenoceptor (O'Dowd et al., 1989). The loss of palmitate often correlates with protein redistribution to the cytosolic subcellular fraction, and agonists which activate G-protein $\alpha_{s}$ accelerate depalmitoylation of the protein (Wedegaertner \& Bourne, 1994; Wedegaertner et al., 1995). There are striking similarities here for eNOS: bradykinin has been shown to promote depalmitoylation of eNOS in $\left[{ }^{3} \mathrm{H}\right]$ palmitate-labeled endothelial cells (Robinson et al., 1995). The loss of the palmitate, and its hydrophobic interactions with the membrane, could be the determining factor in the bradykinin-induced translocation of eNOS to the cytosol. However, other groups, using a very similar system, have failed to identify this agonist-induced 
eNOS-depalmitoylation (Liu et al., 1995) and concluded that palmitoylation plays no major role in the subcellular targeting of eNOS.

The exact role of palmitoylation is complicated by the fact that very little is known about the systems which regulate the reversible palmitoylation of signaling proteins and few of the enzymes are known (Resh, 1996). However, from mutation studies it is known that a myristoylation-deficient mutant eNOS does not undergo palmitoylation (Robinson \& Michel, 1995), possibly because the mutant is not targeted to the membrane, the presumed site for protein palmitoylation (Resh, 1994). This acylation-deficient mutant eNOS, lacking both myristate and palmitate, is entirely cytosolic. A mutant which is deficient in palmitoylation only, has a reduced, but not completely abrogated membrane targeting. This indicates that palmitoylation is not necessary for eNOS membrane association, but increases the efficiency and specificity of the process.

\subsubsection{The eNOS-Caveolin/Calmodulin Cycle}

From the data presented above, a model of the regulatory cycle for eNOS may be inferred.

In resting endothelial cells, the association between eNOS and caveolin suppresses eNOS activity. After agonist activation, the increase in intracellular $\mathrm{Ca}^{2+}$ concentration promotes CaM binding to eNOS and the dissociation of the eNOScaveolin complex. The CaM association causes NO production until the intracellular $\mathrm{Ca}^{2+}$ levels decrease to a point where CaM dissociates from eNOS and the inhibitory eNOS-caveolin complex reforms. The precise details and sequence of events involved in the inactivation and recycling of the enzyme are unclear. This working, speculative 
model has depalmitoylation of eNOS, after prolonged agonist activation, trigger the translocation of eNOS out of the caveolae. In this non-caveolar compartment, the enzyme undergoes phosphorylation, retargeting back to the caveolae, followed by dephosphorylation and palmitoylation. This leads to the inhibitory eNOS-caveolin complex reforming and the return to a resting level of NO production (Michel $e$ t al., 1997; Michel et al., 1997).

However, this is an incomplete model, the enzymes involved in the reversible palmitoylation of proteins are almost entirely unknown and the protein kinases/phosphatases relevant to eNOS regulation are poorly understood. The identity of the intracellular compartment to which eNOS translocates is unknown, although it is likely that some depalmitoylated eNOS translocates to non-caveolar regions of the plasma membrane (Shaul et al., 1996). Other plausible locales include the trans-golgi system, intracellular vesicles derived from caveolae, or a possible interaction with HSP90 (Garcia-Cardena et al., 1998). The interaction between eNOS and caveolin is facilitated by, but not dependent upon, eNOS acylation (Feron et al., 1998). Therefore, agonist-promoted depalmitoylation is unlikely to relieve the tonic inhibition of eNOS activity by caveolin and so it is suggested that palmitoylation may serve to stabilize eNOS on its return to caveolae, promoting the reformation of the eNOS-caveolin complex.

The interactions of eNOS with CaM versus caveolin provide a novel example of the reciprocal regulation of enzyme activity by competing-allosteric protein-protein interactions. This extra level of control of enzyme activity may be particularly important for eNOS in caveolae, where CaM is abundant (Shaul et al., 1996) and 
where even small increases in intracellular $\mathrm{Ca}^{2+}$ could lead to eNOS activation if the eNOS-caveolin complex were not keeping the enzyme in check. Overall, the association between eNOS and caveolin is somewhat of a paradox, providing tonic inhibition of eNOS activity, and yet being required for the efficient activation of eNOS. 


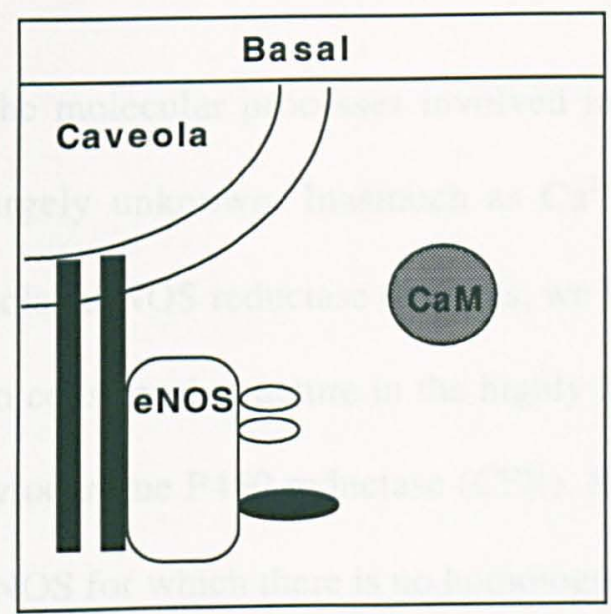

$\uparrow$

Palmit oylation
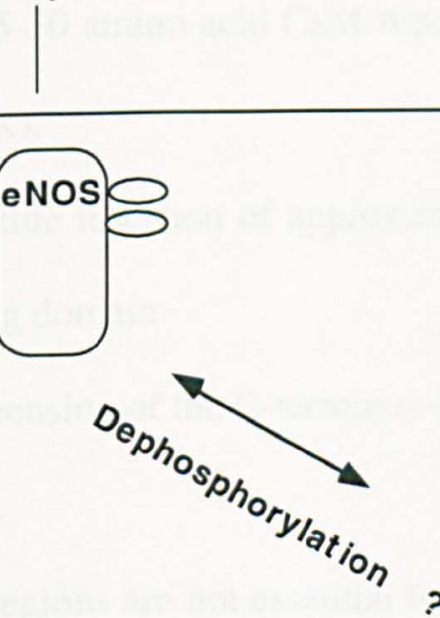

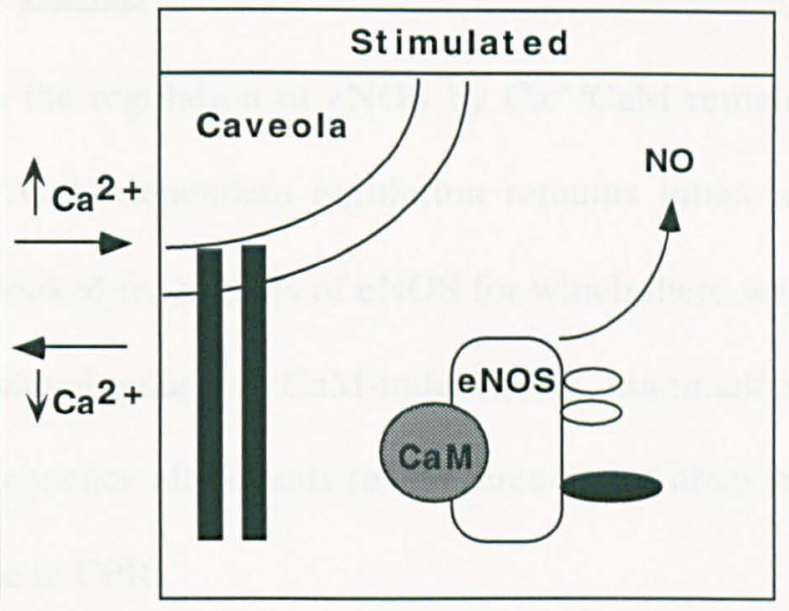

Depalmit oylation
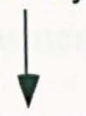

eNOS translocation and recycling

Fig 1.6 The Caveolin-Calmodulin Cycle for eNOS Activation 


\section{$\underline{\text { AIMS }}$}

The molecular processes involved in the regulation of eNOS by $\mathrm{Ca}^{2+} / \mathrm{CaM}$ remain largely unknown. Inasmuch as $\mathrm{Ca}^{2+} / \mathrm{CaM}$-dependent regulation remains intact in isolated NOS reductase domains, we looked for regions of eNOS for which there was no conserved structure in the highly homologous, yet CaM-independent, mammalian cytochrome P450 reductase (CPR). Sequence alignments reveal three major areas of eNOS for which there is no homologue in CPR:

1) The 25-30 amino acid CaM-binding site (analogous to the transmembrane region of $(P R)$.

2) A peptide insertion of approximately 40 amino acids in length within the FMNbinding domain.

3) An extension of the C-terminus of approximately 40 amino acids in length.

As these regions are not essential for the CPR-like reactions of the reductase domain, we hypothesized that they functioned in a coordinated manner with $\mathrm{Ca}^{2+} / \mathrm{CaM}$ to facilitate eNOS regulation. Herein we present studies to investigate the possible regulatory roles of the aforementioned regions of eNOS. 


\section{Chapter 2}

\section{Materials and methods}

\subsection{Materials}

Calmodulin was obtained from Calbiochem (La Jolla, CA) and (6R)-5,6,7,8 tetrahydrobiopterin from Schirks laboratories (Jona, Switzerland). $\left[\mathrm{I}^{125}\right] \mathrm{CaM}$ and $\left[{ }^{32} \mathrm{P}\right]$-labeled ATP were purchased from New England Nuclear (Boston, MA). Terrific broth, isopropyl- $\beta$-D-thiogalactopyranoside (IPTG) and chloramphenicol were purchased from Gibco BRL Products (Gaithersburg, MD). 2'5'-ADP-sepharose 4B was purchased from Pharmacia Biotech (Piscataway, NJ) and GFB membraneclad 96-well microfiltration plates were from Millipore (Bedford, MA). Engineered calmodulin proteins and recombinant HSP90 were kind gifts from Dr. A. Persechini and Dr. N. Pavletich, respectively. Bovine heart cytochrome $c, \beta$-lactoglobulin and all other chemicals were purchased from Sigma and of the best grade available (St. Louis, MO).

\subsection{Mutagenesis of wildtype eNOS}

Mutagenesis of wild type bovine eNOS cDNA was performed using the PCR-based QuiKChange ${ }^{\mathrm{TM}}$ site-directed mutagenesis kit (Stratagene; La Jolla, CA). Briefly, the mutagenesis of eNOS cDNA was carried out in the $\mathrm{pCW}$-eNOS expression vector using two synthetic oligonucleotide primers (forward and reverse) to introduce the desired mutation into wildtype eNOS cDNA. The oligonucleotide primers, each complimentary to opposite strands of the vector, extend during cycling by means of Pfu DNA polymerase. On incorporation of the oligonucleotide primers, a mutated plasmid containing staggered nicks is generated. Following temperature cycling, the product is treated with endonuclease Dpn I (target sequence: $\left.5^{\prime}-\mathrm{G}^{\mathrm{m} 6} \mathrm{ATC}-3^{\prime}\right)$. This 
endonuclease is specific for methylated or hemimethylated DNA and is used to digest the parental DNA template, selecting for the mutation-containing synthesized DNA (DNA isolated from most $E$. coli strains is methylated and therefore susceptible to Dpn I digestion). The nicked vector DNA, containing the mutation, is then ligated and transformed into competent DH5 $\alpha$ E. coli. Introduction of the desired stop codon was confirmed by dideoxynucleotide sequencing, performed by the Cornell University DNA Sequencing Facility. The sequence of the specific primer pairs for each individual mutation are shown in table 2.1.

\section{3 eNOS expression and purification}

Bovine endothelial NOS (eNOS) was purified from Escherichia coli harboring pGroELS and pCW-eNOS expression vectors (Martasek et al., 1996). An overnight culture of pCW-eNOS/pGroELS was used to inoculate 0.5 liter volumes of terrific broth containing ampicillin $(50 \mu \mathrm{g} / \mathrm{ml})$ and chloramphenicol $(35 \mu \mathrm{g} / \mathrm{ml})$ in $2.8 \mathrm{~L}$ Fernbach flasks. Cultures were grown to an $\mathrm{OD}_{600}$ of 0.8 at $37^{\circ} \mathrm{C}$ with shaking at 250 $300 \mathrm{rpm}$. To enhance heme biosynthesis, $\delta$-aminolevulinic acid was added $(0.5 \mathrm{mM}$ final) and cultures grown for another hour. Riboflavin ( $3 \mu \mathrm{M}$ final) and ATP ( $1 \mathrm{mM}$ final) were then added to the cultures and eNOS expression was induced with IPTG ( $0.5 \mathrm{mM}$ final). Cultures were then grown in the dark at $25^{\circ} \mathrm{C}$ and bacteria were harvested after 48 hours by centrifugation. Pellets were stored at $-70^{\circ} \mathrm{C}$ until eNOS purification. Purification of eNOS was accomplished by a modification of the method of Martasek et al.(Martasek et al., 1996). Briefly, eNOS pellets were resuspended in ice-cold buffer A (100 mM Tris- $\mathrm{HCl}, \mathrm{pH}$ 7.6, 1 mM EDTA, $1 \mathrm{mM}$ DTT, 10\% glycerol, $100 \mathrm{mM} \mathrm{NaCl}, 1 \mathrm{mM}$ phenylmethylsulfonyl fluoride, $5 \mu \mathrm{g} / \mathrm{ml}$ pepstatin $\mathrm{A}, 5$ $\mu \mathrm{g} / \mathrm{ml}$ leupeptin and $20 \mathrm{mM}$ CHAPS), lysed by pulsed sonication, and then 


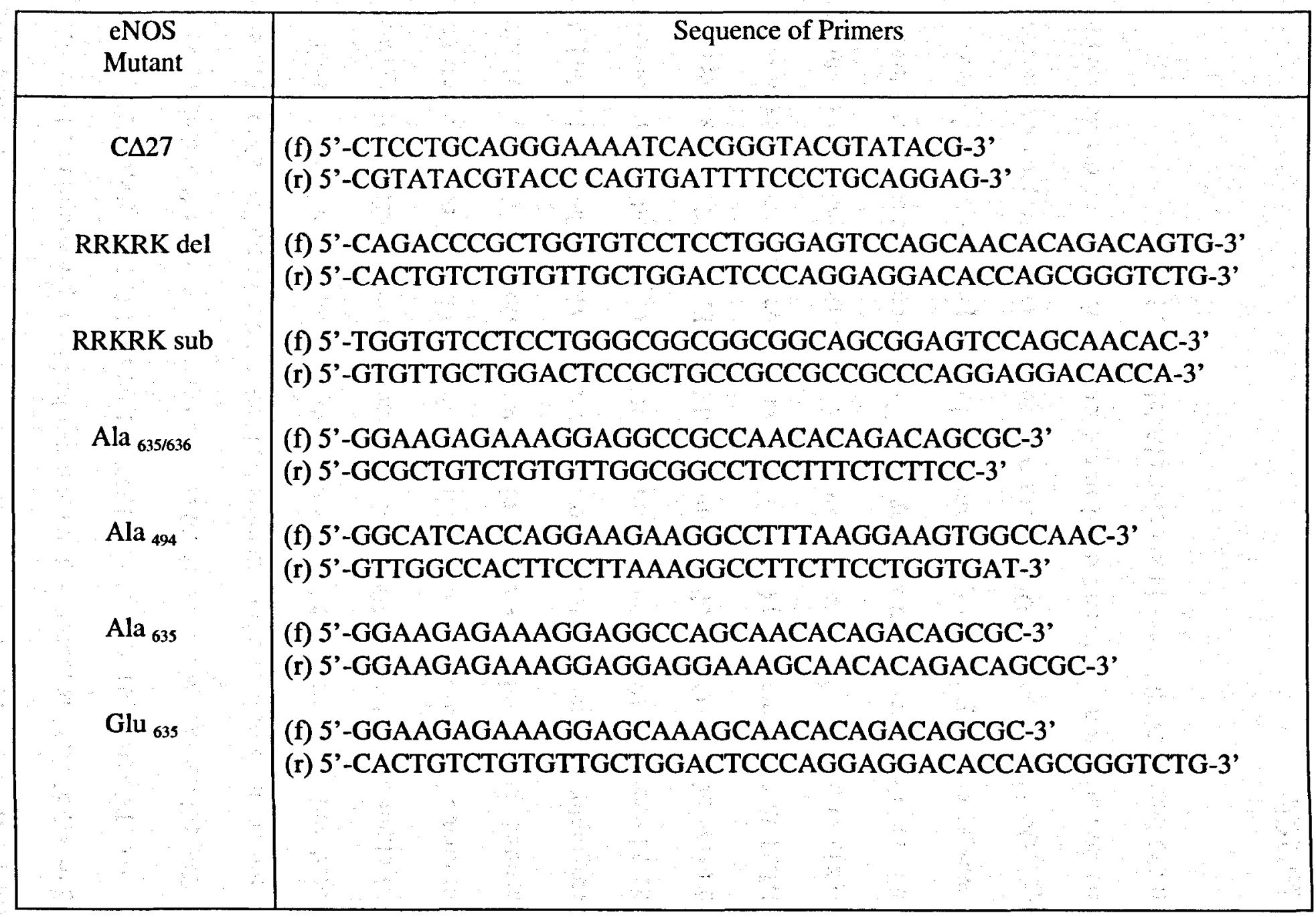

Table. 2.1. Sequences of primer pairs for individual eNOS mutations. $(f=$ forward, $r=r e v e r s e)$. 
centrifuged to sediment cell debris $(120,000 \times \mathrm{g}$, for one hour). The supernatant was applied to a 2' 5'-ADP sepharose 4B column that had been pre-equilibrated in buffer B (100 mM Tris-HCl, pH 7.6, $0.1 \mathrm{mM}$ EDTA, $1 \mathrm{mM}$ DTT, 10\% glycerol, $100 \mathrm{mM}$ $\mathrm{NaCl})$. The column was washed with 20 column volumes of buffer $\mathrm{B}$ and again with 20 column volumes of buffer B containing high salt $(600 \mathrm{mM} \mathrm{NaCl})$. eNOS Protein was eluted with high salt buffer B that additionally contained $10 \mathrm{mM}$ NADPH. The NADPH eluate was diluted to $100 \mathrm{mM} \mathrm{NaCl}$ concentration in buffer $\mathrm{B}$ by repeated concentration/dilution using a centrifugal filter device (Biomax-100K, Millipore). The concentrated and desalted NADPH eluate was further purified by affinity chromatography on a calmodulin-sepharose column as previously described (Black \& Ortiz de Montellano, 1995). Purification of nNOS was achieved by an identical method using $\mathrm{pCW}-\mathrm{nNOS} / \mathrm{pGroELS}$ transformed BL21 E. coli. iNOS was prepared using BL21 E. coli containing pCW/iNOS and an additional calmodulin expression vector (Xia et al., 1998). Due to the cotranslational incorporation of calmodulin, purification of iNOS was performed using the 2'5'-ADP-sepharose step only. Protein purity was assessed by SDS-polyacrylamide gel electrophoresis using Coomassie staining. Quantification of NOS protein was determined spectrophotometrically, based on an extinction coefficient of $74 \mathrm{mmol}^{-1} \mathrm{~cm}^{-1}$ for $\mathrm{A}_{444}-\mathrm{A}_{550}$ of the dithionitereduced CO-bound eNOS heme-chromophore (Adak et al., 1999).

\subsection{SDS-PAGE}

SDS-PAGE was carried out using precast $8-16 \%$ polyacrylamide gels (Novex, San Diego. CA) and were stained with Coomassie blue. Samples were prepared in gel loading buffer (63 mM Tris-HCl, $10 \%$ glycerol, $2 \%$ SDS, $0.0025 \%$ bromophenol blue, $\mathrm{pH} 6.8$ ) and denatured by boiling $\left(95^{\circ} \mathrm{C}, 15 \mathrm{~min}\right)$. Electrophoresis was 
performed in SDS running buffer ( $25 \mathrm{mM}$ Tris- $\mathrm{HCl}, 192 \mathrm{mM}$ glycine, $0.1 \% \mathrm{SDS}, \mathrm{pH}$ 8.3) at a constant amperage of $30 \mathrm{~mA}$.

When NOS dimer stability was assessed by low-temperature SDS-PAGE, samples were prepared in gel loading buffer, but were not denatured by boiling. Electrophoresis was performed in SDS running buffer and the electrophoresis tank was placed in an ice-bath. Separation was performed at a constant voltage of $185 \mathrm{~V}$.

\subsection{Spectrophotometry of the CO-bound heme chromophore}

Spectral data were recorded on a Beckman DU-7500 spectrophotometer. Scans of dithionite-reduced CO-bound proteins were taken in CO-saturated buffer (40 mM HEPES, pH 7.6, containing $10 \%$ glycerol, $1 \mathrm{mM}$ DTT and $100 \mathrm{mM} \mathrm{NaCl}$ ). The ferrous-CO adduct absorbing at $444 \mathrm{~nm}$ was used to quantitate the hemoprotein content using an extinction coefficient of $74 \mathrm{mmol}^{-1} \mathrm{~cm}^{-1}\left(\mathrm{~A}_{444}-\mathrm{A}_{550}\right)$ (Adak et al., 1999).

\subsection{Assays of enzyme activity}

\subsubsection{Griess assay}

NO production was deduced from accumulation of the stable oxidation product nitrite, as determined using the Griess assay. Assays were carried out in a 96well microplate format with $100 \mu \mathrm{l}$ total sample volume. All wells contained Larginine $(1 \mathrm{mM})$, calmodulin $(100 \mathrm{nM}), \mathrm{CaCl}_{2}(100 \mu \mathrm{M}), \mathrm{BH} 4(10 \mu \mathrm{M}), \operatorname{Tris}-\mathrm{HCl}(50$ $\mathrm{mM}, \mathrm{pH}$ 7.6) and eNOS at specific concentrations. Reactions were initiated by the addition of NADPH $(1 \mathrm{mM})$. After one hour at $37^{\circ} \mathrm{C}, 10 \mu \mathrm{l}$ of lactate dehydrogenase was added (20 $\mu \mathrm{l} \mathrm{LDH}$ slurry in $0.5 \mathrm{ml}$ of $500 \mathrm{mM}$ pyruvate) and samples were 
incubated at $37^{\circ} \mathrm{C}$ for $15 \mathrm{~min}$. Griess reagent (freshly made $1: 1$ mix of $1 \%$ sulfanilamide in $5 \%$ phosphoric acid and $0.1 \% \mathrm{~N}$-(1-napthyl)-ethylenediamine) was then added as a $100 \mu \mathrm{l}$ volume and $A_{550}$ was determined within $10 \mathrm{~min}$. The level of nitrite in samples was assessed by comparison with sodium nitrite standards.

For experiments that assess the $\mathrm{Ca}^{2+}$-dependence of NO synthesis, reactions were carried as above, except that reaction buffer was substituted (10 mM MOPS, 100 $\mathrm{mM} \mathrm{KCl}, \mathrm{pH} 7.2$ ), containing varying ratios of EGTA- $\mathrm{Ca}^{2+}$ to give desired $\mathrm{Ca}^{2+}$ concentrations (described separately).

\subsubsection{Assay of NOS activity by myoglobin or NADPH oxidation}

NOS activity was measured in 96 -well microtiter plates at $37^{\circ} \mathrm{C}$ based on the kinetics of NADPH consumption or the oxidation of $\mathrm{Fe}^{2+}$-myoglobin to $\mathrm{Fe}^{3+}$ myoglobin (Gross, 1996). For NADPH consumption measurements assay mixtures contained L-arginine (1 mM), calmodulin (100 nM), $\mathrm{CaCl}_{2}(100 \mu \mathrm{M}), \mathrm{BH} 4(10 \mu \mathrm{M})$, Tris- $\mathrm{HCl}(50 \mathrm{mM}, \mathrm{pH} 7.6)$ and eNOS at specific concentrations. Reactions were initiated by the addition of 20 pmole of nNOS, 10 pmole of eNOS, or $15 \mu \mathrm{g}$ of rat iNOS-rich cytosol. NADPH consumption was determined from the rate of decrease in $A_{340}$, measured at 15 second intervals for a period of $30 \mathrm{~min}$ in a kinetic microplate spectrophotometer (Molecular Devices; Menlo Park, CA). The rate of decline in $A_{340}$ measured when NOS was omitted from incubates was subtracted from all values. Samples in which iNOS activity was measured based $\mathrm{Fe}^{2+}$-myoglobin oxidation were prepared as above, but additionally contained $15 \mu \mathrm{g}$ of iNOS-rich cytosol and $\mathrm{Fe}^{2+}$ myoglobin $(40 \mu \mathrm{M}) . \mathrm{Fe}^{2+}$-myoglobin oxidation was determined by monitoring the change in $\mathrm{A}_{420}$ in a kinetic 96 -well microplate spectrophotometer. Preparation of $\mathrm{Fe}^{2+}$ - 
myoglobin was attained by treatment with sodium dithionite and purification by size exclusion chromatography using a G-25 column (Pharmacia).

\subsubsection{Reduction of cytochrome $c$ and ferricyanide}

Assays of eNOS reductase domain activity were carried out in 96-well plate format with a total reaction volume of $100 \mu 1$. Reaction progress was monitored continually at $15-\mathrm{sec}$ intervals at $\mathrm{A}_{550}$ and $\mathrm{A}_{405}$, for cytochrome $\mathrm{c}$ and ferricyanide reduction, respectively. Assay buffer was added to wells (40 mM HEPES, pH 7.6, 0.1 $\mathrm{mg} / \mathrm{ml}$ bovine serum albumin, $250 \mathrm{nM} \mathrm{CaM,} \mathrm{0.6mM} \mathrm{EDTA,} 10$ units/ml superoxide dismutase, and 10 units/ml catalase) and either $100 \mu \mathrm{M}$ bovine heart cytochrome $\mathrm{c}$ or $1 \mathrm{mM}$ potassium ferricyanide. To assess the dependence on $\mathrm{CaM}$ binding, maximum CaM binding was elicited by addition of $0.83 \mathrm{mM} \mathrm{Ca}^{2+}$ to relevant samples. Following addition of the desired quantity of eNOS, substrate reduction was initiated by addition of NADPH (100 $\mu \mathrm{M}$ final concentration).

\subsubsection{Calcineurin Activity Measurement}

Activity of the CaM-dependent phosphatase, calcineurin (protein phosphatase 2B) was monitored in a 96-well kinetic microplate spectrophotometer at $37^{\circ} \mathrm{C}$, based on the rate of hydrolysis of the artificial substrate p-nitrophenyl phosphate (pNPP). Incubation mixtures contained Tris- $\mathrm{HCl}(50 \mathrm{mM}, \mathrm{pH} 7.6), \mathrm{CaCl}_{2}(100 \mu \mathrm{M}), \mathrm{CaM}$ (100 $\mathrm{nM}), 2$-mercaptoethanol $(0.1 \%)$ and $\mathrm{pNPP}(40 \mathrm{mM})$ in a $100 \mu$ l reaction volume. Reactions were initiated by the addition of calcineurin ( 40 pmole), and activity was monitored kinetically at $A_{40 s}$ for $60 \mathrm{~min}$ at 15 second intervals. Assay blanks additionally contained EGTA $(10 \mathrm{mM})$, resulting in $>90 \%$ inhibition of activity. 


\subsection{Assays of NOS-ligand binding}

\subsubsection{Assay of [ $\left.{ }^{125} \mathrm{I}\right]-$ Calmodulin Binding to NOS}

CaM binding to eNOS was analyzed in 96-well microfiltration plates containing GFB membranes that had been prewashed with binding buffer (10 $\mathrm{mM}$ MOPS, $100 \mathrm{mM} \mathrm{KCl}, 100 \mu \mathrm{M} \mathrm{CaCl}_{2}$, and $\beta$-lactoglobulin $0.5 \%, \mathrm{pH}$ 7.2). Incubations contained binding buffer, $\mathrm{BH}_{4}(10 \mu \mathrm{M})$ and DTT $(1 \mathrm{mM})$ supplemented with desired concentrations of $\left.{ }^{[25} \mathrm{I}\right]-\mathrm{CaM}$ in a total volume of $100 \mu \mathrm{l}$. In some cases, $\mathrm{Ca}^{2+}$-buffer solutions were additionally added. Binding reactions were initiated by the addition of eNOS at specific concentrations and proceeded for 20 minutes at $25^{\circ} \mathrm{C}$. Binding was terminated by vacuum filtration and filters were washed twice with $100 \mu$ lof ice-cold assay buffer. Filtration plates were air-dried under vacuum and $25 \mu \mathrm{l}$ of scintillation cocktail was added to each well (Optiphase Supermix, Wallac). Bound radioactivity was determined in a Microbeta Plus 96-well liquid scintillation counter (Wallac). Specific binding of $\mathrm{CaM}$ was defined as the component of total binding, which was lost when samples were co-incubated with excess calcium-chelator ( $5 \mathrm{mM} \mathrm{EGTA)} \mathrm{or}$ 1000-fold molar excess of unlabeled CaM.

\subsection{2 $\left.{ }^{125} \mathrm{I}\right]-\mathrm{CaM}$ association and dissociation rate measurement}

Association of CaM to NOS was determined as described above, except that incubations were terminated after the desired interval by rapid filtration. For the estimation of dissociation rates, NOS was pre-incubated $(15 \mathrm{~min})$ with $\left[{ }^{125} \mathrm{I}\right]-\mathrm{CaM}$, prior to addition of a 3000 -fold molar excess of unlabeled $\mathrm{CaM}$, to initiate dissociation. The reaction was terminated after the desired interval by rapid filtration. 


\subsubsection{Assay of $\left[{ }^{3} \mathrm{H}\right]-\mathrm{N}^{\mathrm{G}}$ Nitro-L-Arginine binding}

Assays of $\left[{ }^{3} \mathrm{H}\right] \mathrm{N}^{\mathrm{G}}$ Nitro-L-arginine binding were performed in $96-$ well polyvinylidene difluoride microfiltration plates (Millipore, Bedford, MA) in a similar manner to that described for $\left[{ }^{125} \mathrm{I}\right]-\mathrm{CaM}$. Reaction mixture was identical to that used for $\left[{ }^{125} \mathrm{I}\right]-\mathrm{CaM}$, except $\left[{ }^{3} \mathrm{H}\right] \mathrm{N}^{\mathrm{G}}$ Nitro-L-arginine was substituted for $\left[{ }^{125} \mathrm{I}\right]-\mathrm{CaM}$. Subsequent equilibration and washing steps were performed as decribed.

\subsubsection{Preparation of Calcium Solutions}

Stock solutions of "zero" free $\mathrm{Ca}^{2+}(10 \mathrm{mM}$ MOPS, $100 \mathrm{mM} \mathrm{KCl}, 10 \mathrm{mM}$ EGTA, $\mathrm{pH} 7.2$ ) and $40 \mu \mathrm{M}$ free $\mathrm{Ca}^{2+}(10 \mathrm{mM}$ MOPS, $100 \mathrm{mM} \mathrm{KCl}, 10 \mathrm{mM}$ EGTA, $10 \mathrm{mM} \mathrm{CaCl}_{2}, \mathrm{pH}$ 7.2) were prepared. Solutions of defined free $\mathrm{Ca}^{2+}(0-40 \mu \mathrm{M})$ were then prepared by mixing varying ratios of the two solutions (Fabiato \& Fabiato, 1979). The free- $\mathrm{Ca}^{2+}$ concentration was quantified by ratiometric fluorometry using fura-2.

\subsection{PKA-mediated phosphorylation of eNOS}

NOS (10pmoles) was incubated at $25^{\circ} \mathrm{C}$ for the indicated time period in PKA phosphorylation buffer $(2 \mathrm{mM} \mathrm{MgCl}, 100 \mu \mathrm{M} \mathrm{CaCl}, 50 \mathrm{mM}$ Tris- $\mathrm{HCl}, \mathrm{pH}$ 7.6) containing CaM (100nM), $\mathrm{BH}_{4}(10 \mu \mathrm{M})$, PKA (5units) and $\gamma$ - labeled ${ }^{32}$ P-ATP $(100 \mu \mathrm{M})$ in a final reaction volume of $100 \mu \mathrm{l}$. At relevant time points, aliquots $(20 \mu \mathrm{l})$ were removed, mixed 1:1 with SDS-gel loading buffer and heated $\left(95^{\circ} \mathrm{C}, 10 \mathrm{mins}\right)$ to stop the reaction. Samples were then run on $8-16 \%$ SDS polyacrylamide gels and coomassie stained. Autoradiography was performed on dried gels to assess ${ }^{32} \mathrm{P}$ incorporation into eNOS and appropriate gel slices were excised for quantification by liquid scintillation counting. 
For studies assessing the effect of phosphorylation on eNOS activities, phosphorylated eNOS was prepared in 400 pmole quantities $(1$ pmole/ $\mu \mathrm{l})$ and then frozen for subsequent use in NOS assays. Phosphorylation was confirmed by phosphate incorporation using ${ }^{32} \mathrm{P}$-labeled ATP in a scaled down sample, under identical conditions.

\subsection{Limited proteolysis of NOS proteins}

Limited proteolysis was performed on incubates containing $40 \mathrm{pmol}$ of recombinant NOS purified from E. Coli. Samples were preincubated at room temperature for $15 \mathrm{~min}$ in a $100 \mu \mathrm{l}$ volume containing: $50 \mathrm{mM}$ Tris, $\mathrm{pH} 7.6,1 \mathrm{mM}$ DDT, $10 \mu \mathrm{M} \mathrm{CaM}, 100 \mu \mathrm{M} \mathrm{CaCl}_{2}$, with or without $10 \mathrm{mM}$ EGTA. Proteolysis was initiated by the addition of 20 microunits of L-1-tosyl-amido-2-phenylethyl chloromethyl ketone-immobilized trypsin (Sigma) per pmol of NOS. Samples $25 \mu \mathrm{l}$ were collected after $0,5,10$ and $20 \mathrm{~min}$, and proteolysis was terminated by boiling with an equal volume of $2 X$ SDS gel-loading buffer. Peptide products were resolved by gradient SDS-polyacrylamide gel electrophoresis and visualized by staining with Coomassie Blue. Accurate molecular mass determination of tryptic fragments was performed by mass spectrometry at the Weill Medical College Mass Spectrometry Core Facility by matrix-assisted laser desorption ionization time of flight (MALDITOF) mass spectrometry.

\subsection{Protein modeling}

Molecular modeling of the FMN binding module of nitric oxide synthase isoforms was done using the Insight II, Homology and Discover programs from 
Biosym (BIOSYM/Molecular Simulations, San Diego, CA) running on a Silicon Graphics Indigo 2 workstation. After alignment of NOS sequences with homologous FMN binding proteins of known structure, structurally conserved region (SCR) boxes were created, corresponding to conserved regions of secondary structure and regions directly involved in FMN binding. These regions were characterized by high positive scores as evaluated by Dayhoff's mutation matrix (Dayhoff et al., 1983). After assignment of coordinates in the SCR regions, the loop regions between the SCR boxes were modeled by searching the Brookhaven protein database. The crude model structure was relaxed to a sterically and energetically reasonable state using the Discover program (BIOSYM/Molecular Simulations) for molecular mechanics and dynamics calculations. This includes splice repair to remove unrealistic structural features at SCR-loop junctions and N-terminal extensions, and structural optimization to remove steric overlaps. Energy minimizations were begun using the steepest descent method; this was replaced by the conjugate gradient method as convergence was approached.

\subsection{Peptide synthesis}

Synthetic peptides were obtained from SynPep (Dublin, CA) and other commercial suppliers. Purity was assessed to exceed $80 \%$ as evaluated by high performance liquid chromatography and mass spectrometry. Predominant impurities differed from the desired product by one amino acid residue, the result of incompletely coupled synthesis. 


\subsection{Electrospray Ionization Mass Spectrometry}

Electrospray ionization mass spectrometry using selected ion recording (SIRMS) was performed at the biopolymer mass spectrometry research core facility at Weill Medical College. Analysis was performed using a Quatro II (Micromass, U.K.) ESI- mass spectrometer 


\section{Chapter 3}

IDENTIFICATION AND CHARACTERIZATION OF AN

AUTOINHIBITORY CONTROL ELEMENT WITHIN THE FMN-BINDING

DOMAIN OF CALCIUM-REGULATED ISOFORMS OF NITRIC OXIDE

\section{SYNTHASE}

\subsection{Introduction}

Nitric oxide synthases (NOSs) comprise a family of three calmodulindependent isoforms that are functionally distinguished by their modes of regulation. The two constitutively expressed isoforms of NOS (cNOSs), originally purified from neuronal (nNOS) and endothelial cells (eNOS), remain dormant until calcium/calmodulin $\left(\mathrm{Ca}^{2+} / \mathrm{CaM}\right)$ binding is elicited by transient elevations in intracellular $\mathrm{Ca}^{2+}$ (Nathan \& Xie, 1994; Sessa, 1994). This $\mathrm{Ca}^{2+}$-dependent mode of regulation provides pulses of NO for the moment-to-moment modulation of vascular tone/neurosignaling. In contrast, the activity of the imunostimulant-induced isoform of NOS (iNOS) is $\mathrm{Ca}^{2+}$-independent, providing continuous high-output $\mathrm{NO}$ generation for the purpose of host defense. This $\mathrm{Ca}^{2+}$-independence is due to the remarkably high affinity CaM-binding exhibited by iNOS, indeed, extreme conditions (boiling in $1 \%$ sodium dodecyl sulphate) are required to cause the dissociation of CaM from iNOS (Cho et al., 1992).

Whether a given isoform binds $\mathrm{CaM}$ in a $\mathrm{Ca}^{2+}$-dependent or -independent manner has been assumed to be solely due to inter-isoform differences in the amino acid sequence of the 20-25 amino acid CaM-binding site. However, this view has proven to be restrictive, given that chimeric eNOS and nNOS which have been 
engineered to contain the CaM-binding site of iNOS, still require $\mathrm{Ca}^{2+}$ for full activity (Ruan et al., 1996; Venema et al., 1996). Therefore regions other than the CaMbinding site must contribute, at least in part, to the $\mathrm{Ca}^{2+}$-dependence exhibited by the various NOS isoforms. Because regulation of enzyme systems by $\mathrm{Ca}^{2+} / \mathrm{CaM}$ typically involves the displacement of an intrinsic autoinhibitory polypeptide (Jarrett \& Madhavan, 1991), we hypothesized that the binding of $\mathrm{Ca}^{2+} / \mathrm{CaM}$ to $\mathrm{cNOSs}$ may similarly trigger activation by displacing an autoinhibitory control element. This chapter describes studies in which we identify a 40-45 amino acid insertion peptide within the FMN-binding domain of cNOSs, and investigate its role in enzyme regulation. 


\subsection{Results}

\subsubsection{Expression, purification and characterization of eNOS, nNOS and iNOS}

\subsubsection{Expression and Purification}

In order to investigate the regulation of the NOS family of proteins it was first necessary to express and purify the various NOS isoforms for in in vitro characterization. Expression and purification were accomplished using an established E. coli based expression system (Martasek et al., 1996; Roman et al., 1995) and purification was accomplished using two sequential chromatography separation steps, as described in methods. Proteins were purified to $\approx 90 \%$ homogeneity, as assessed by SDS-polyacrylamide gel electrophoresis (SDS-PAGE) (Fig. 3.1) and were of the predicted molecular weights for iNOS (130 kDa), nNOS (160 kDa) and eNOS (135 kDa) (Sessa, 1994).

\subsubsection{Characterization}

These purified NOSs were then characterized in order to assess functionality. As all NOS proteins are obligate homodimers, the ability of the purified proteins to form stable dimers was assessed using low temperature SDS-PAGE. As is shown in Fig. 3.2, each of the expressed NOS isoforms existed predominantly in homodimeric form. Functional NOS proteins contain a heme-group (protoporphyrin IX) held in place by planar linkages to four pyrole nitrogens and a conserved cysteine residue in the oxygenase domain (penta-coordinate). During NO catalysis ferrous iron binds molecular oxygen as the sixth axial ligand. Functional incorporation of heme into NOS proteins can be assessed by spectrophotometry using the ability of CO-bound 
reduced heme to produce a cytochrome P450-like absorption peak at $446 \mathrm{~nm}$ (Fig 3.3, panels A, B and C, for iNOS, nNOS and eNOS, respectively). All of the purified proteins exhibited a P450-like spectrum, indicative of functional heme incorporation.

To assess the extent to which recombinant NOSs could in fact synthesize NO, specific activity of the proteins was measured using the Griess assay for nitrite/nitrate accumulation. Determinations were based upon the average of at least two preparations of each isoform, assayed in triplicate. The NOSs displayed specific activity values of $660 \pm 53,516 \pm 83$ and $135 \pm 17 \mathrm{nmoles} / \mathrm{min} / \mathrm{mg}$ NOS protein for iNOS, nNOS and eNOS, respectively (Fig. 3.4). These specific activities compare well with the optimal values from the literature (Martasek et al., 1996; Roman et al., 1995; Stuehr et al., 1991; Wu et al., 1996). Due to the differences in regulation by $\mathrm{Ca}^{2+} / \mathrm{CaM}$, an important functional test was to establish the $\mathrm{Ca}^{2+}$-dependence for activation by CaM of the three NOS isoforms. As shown in Fig. 3.5, iNOS displayed full activity at all levels of free $\mathrm{Ca}^{2+}$ tested. In contrast, activity of cNOSs were dependent upon elevated levels of free $\mathrm{Ca}^{2+}, \mathrm{EC}_{50}$ values for $\mathrm{eNOS}$ and $\mathrm{nNOS}$ were $460 \mathrm{nM}$ and $410 \mathrm{nM}$, respectively (Fig. 3.5).

The above studies confirmed that purified NOS proteins were dimeric, contained heme in the correct orientation, produced $\mathrm{NO}$ and were regulated by $\mathrm{Ca}^{2+}-$ levels in the predicted fashion. These findings demonstrated the suitability of purified recombinant NOS isoforms for subsequent investigations of enzymatic regulation. 
eNOS nNOS iNOS

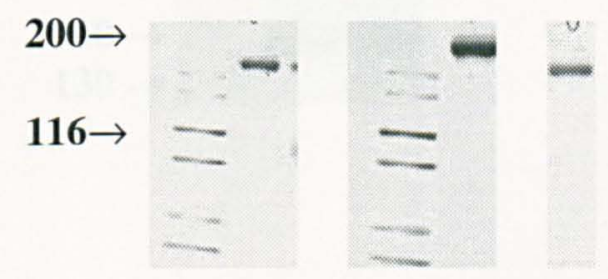

Fig. 3.1. SDS-polyacrylamide gel electrophoresis of purified recombinant NOSs. Recombinant NOS proteins were all purified to $>90 \%$ homogeneity and showed approximate molecular weights of $135 \mathrm{kDa}(\mathrm{eNOS}), 160 \mathrm{kDa}$ (nNOS) and $130 \mathrm{kDa}$ (iNOS). 


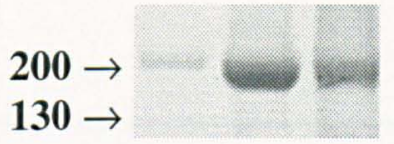

Fig. 3.2. Stability of NOS homodimer by low-temperature SDSpolyacrylamide gel electrophoresis. Low temp SDS-PAGE was performed as described in Methods. The apparent molecular weight of > $200 \mathrm{kDa}$ indicated the formation of stable NOS homodimers for all three isoforms. 

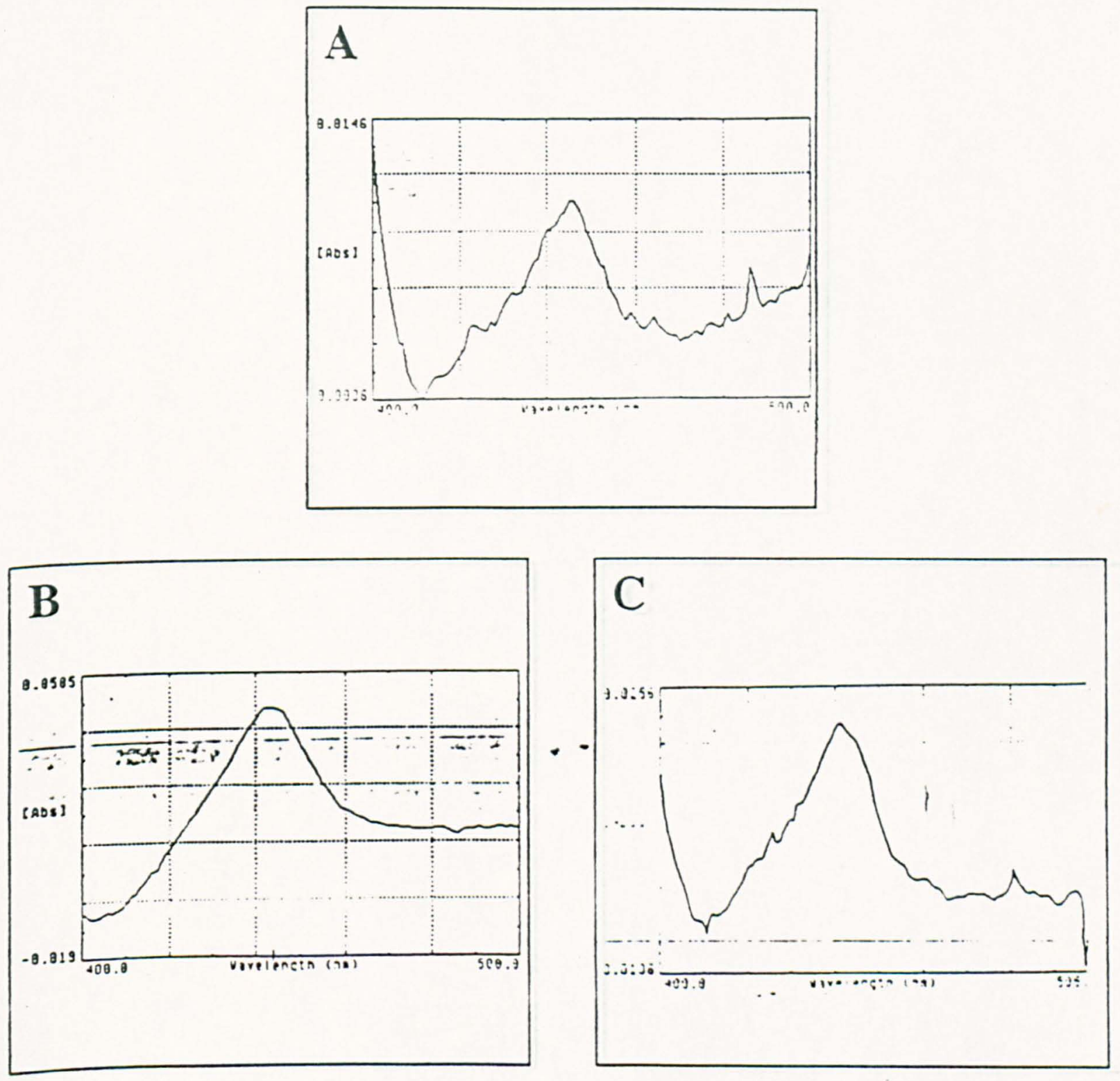

Fig. 3.3. P450 spectra of eNOS, nNOS and iNOS. The CO-bound, dithionite reduced spectra of eNOS (panel A), nNOS (panel B) and iNOS (panel C) were obtained as described in methods. 


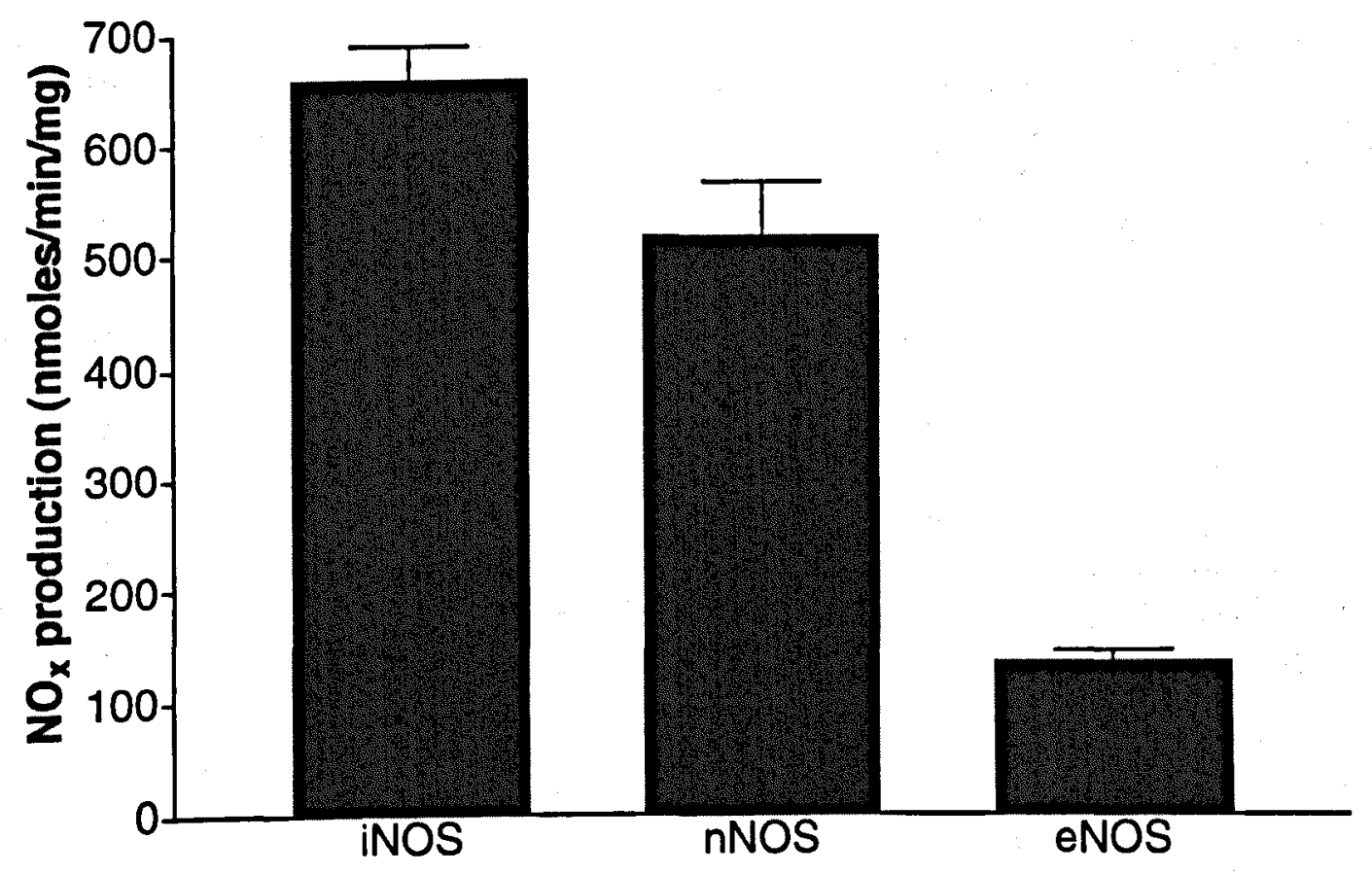

Fig. 3.4. Rates of NO synthesis of the three NOS isoforms. All experiments were carried out using 8 pmoles of NOS, $250 \mathrm{nM}$ CaM and an optimal $\mathrm{Ca}^{2+}$ concentration $(100 \mu \mathrm{M})$. CaM-free measurements of activity were obtained in the presence of $5 \mathrm{mM}$ EGTA and rates are the mean of two separate preparations of each enzyme assayed in triplicate \pm SEM. 


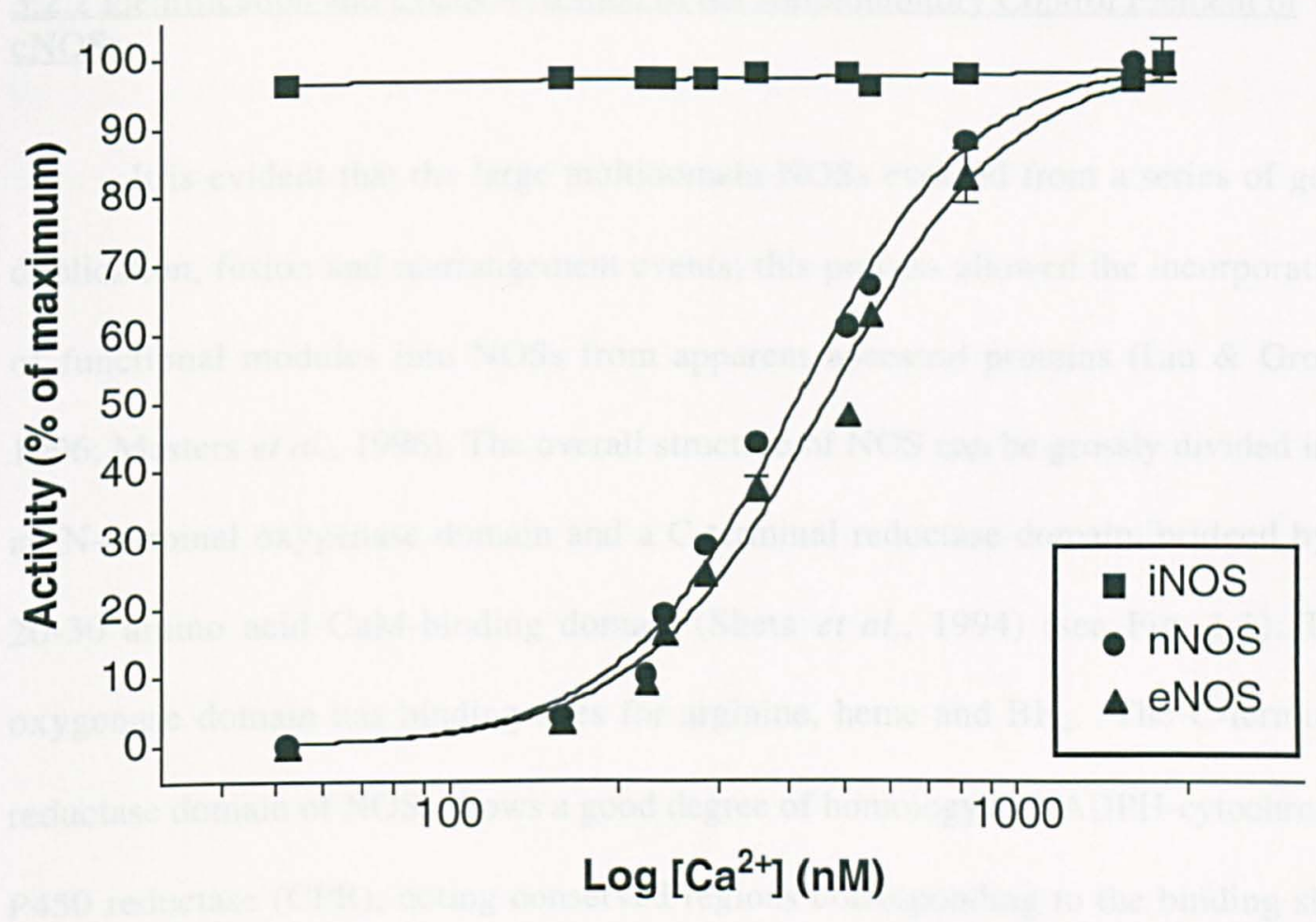

Fig. 3.5. $\mathrm{Ca}^{2+}$-dependence of the three isoforms of NOS. NOS (8 pmoles) in the presence of $100 \mathrm{nM} \mathrm{CaM}$ was incubated in a range of $\mathrm{Ca}^{2+}$ concentrations, as described in Methods. NO synthesis was assessed using the Griess assay and blank activity was determined in the presence of $10 \mathrm{mM}$ EGTA. Activity is expressed as a percentage of the maximum activity obtained at the highest $\mathrm{Ca}^{2+}$-concentration used $(\approx 1.8 \mu \mathrm{M})$. Values shown are the mean of two separate preparations assayed in triplicate \pm SEM. 
3.2.2 Identification and Characterization of the Autoinhibitory Control Element of cNOSs

It is evident that the large multidomain NOSs evolved from a series of gene duplication, fusion and rearrangement events; this process allowed the incorporation of functional modules into NOSs from apparent ancestral proteins (Liu \& Gross, 1996; Masters et al., 1996). The overall structure of NOS can be grossly divided into an $\mathrm{N}$-terminal oxygenase domain and a $\mathrm{C}$-terminal reductase domain, bridged by a 20-30 amino acid CaM-binding domain (Sheta et al., 1994) (see Fig. 1.1). The oxygenase domain has binding sites for arginine, heme and $\mathrm{BH}_{4}$. The $\mathrm{C}$-terminal reductase domain of NOSs shows a good degree of homology to NADPH-cytochrome P450 reductase (CPR), noting conserved regions corresponding to the binding sites for FAD, FMN and NADPH (Bredt et al., 1991). Structural homologues of the FMNbinding modules of both NOSs and CPR can be found in the bacterial flavodoxins, small FMN-binding proteins that function as electron carriers (Porter, 1991). Similarly, homologies for the FAD and NADPH binding domains can be found in chloroplast ferredoxin-NADP ${ }^{+}$reductase and other related $\mathrm{FAD}$-containing proteins.

\subsubsection{Sequence Alignments}

High resolution crystal structures of at least five flavodoxins have been solved by X-ray diffraction (Watenpaugh et al., 1973), revealing three distinct regions that are involved in binding of the FMN moiety. Although only one of these regions was initially identified in nNOS (Bredt et al., 1991), each has a corresponding homologue in NOSs. Homology-based alignments of the FMN-binding domains of NOSs and flavodoxins performed by Dr. J.C. Salerno (Salerno et al., 1997) identified these 
regions in each NOS isoform. This was followed by the identification of conserved secondary structural elements in NOS that correspond to elements present in the flavodoxins, primarily by their homology based on mutational matrix criteria (Dayhoff et al., 1983).

Fig. 3.6 (Panel A) shows the structurally based alignment of representative members of the NOSs, bacterial flavodoxins and CPRs, indicating the conserved regions that participate in the binding of FMN. Despite conspicuous sequence homology, it is obvious that a major insertion of approximately 45 amino acids is uniquely present within the mammalian cNOSs. Inspection of the protein database reveals that this insertion is conserved in the FMN-binding domains of all reported cNOSs of both mammalian and invertebrate origin (Fig. 3.6, panel B). The insertions present in the FMN-binding domains of the cloned insect cNOSs from Rhodnius prolixus (Yuda et al., 1996) and Drosophila melanogaster (Regulski \& Tully, 1995) are somewhat larger than their mammalian counterparts (54-63 amino acids), but the sequences still share regions of conservation with the smaller mammalian insertion. Expanding the alignment to include the dozens of known related FMN-binding proteins reveals that only cNOSs contain such an insertion (alignments not shown). Furthermore, like the cNOSs, the FMN-binding domain of the $\mathrm{Ca}^{2+}$-independent iNOS shows marked homology to the FMN-binding motifs of the related proteins, but lacks the peptide insertion present within the cNOSs. Hence, the insert correlates with $\mathrm{Ca}^{2+} / \mathrm{CaM}$ control and represents the single most prominent difference between cNOS and iNOS amino acid sequences, when considered over their entire length. 


\subsubsection{Structural Modeling}

The lack of structural information concerning the reductase domain of any of the NOS isoforms is a hindrance to the elucidation of the function of this peptide insertion. However, the availability of solved X-ray structures for flavodoxins allows us to position the insertion in three-dimensions relative to the CaM-binding site. Homology-based molecular models have been constructed for the FMN-binding domains of cNOSs, iNOS, and CPR (for which a crystal structure has now been solved), which could be relaxed to a sterically and energetically reasonable state. After relaxation of these FMN-binding domain models, no steric overlaps were evident, and energies were approximately $-300 \mathrm{kcal}$, comparable or lower than that of the reference flavodoxin crystal structure.

As shown in Fig. 3.7 (panel A), the backbone structure of iNOS and CPR are virtually superimposable on the backbone of the solved crystal structure of the closest solved structural homologue to the FMN-binding domain of NOSs (Desulfovibrio vulgaris flavodoxin). The structure, a Rossmann fold motif (Rossmann et al., 1974), is a five stranded parallel $\beta$-sheet with the FMN-binding site along one edge. Homology predicts that two aromatic residues in murine iNOS, $\mathrm{Phe}^{587}$ and $\mathrm{Tyr}^{625}$, are stacked above and below the FMN ring system.

Fig. 3.7 (panel B) shows the corresponding backbone structure of eNOS (nNOS is extremely similar, but is not shown); the eNOS backbone is essentially superimposable over the homologues shown in the previous panel. However, the insertion, having no structural homologue, is shown projecting from the upper edge of the sheet opposite the FMN-binding site. Structurally, it corresponds to the 
replacement of a tight 5-10 residue $\alpha \rightarrow \beta$ loop with a 45 amino acid structure, the insertion being approximately one third of the overall size of the FMN-binding module. Unfortunately, no conformation can be assigned to the insertion region due to the lack of a resolved homologue; the mock structure shown is provided merely to demonstrate relative position and size.

The CaM-binding site is immediately $\mathrm{N}$-terminal to the FMN-binding domain (this location is tipped white in panel A). In the CaM-bound conformation, the CaMbinding site would predictably adopt a helical conformation (O'Neil \& De Grado, 1990; Vorherr et al., 1993) and steric constraints suggest that it extends almost directly away from the FMN-binding module. Fig. 3.8 shows models of the FMNbinding domains of iNOS (panel A) and eNOS (panel B) with CaM (yellow ribbon, derived from the structure of Vorherr et al. (Vorherr et al., 1993)) positioned above the N-terminal strand of the FMN-binding domain. There are 7-8 residues between the end of the CaM recognition site proper and the start of the initial $\beta$-sheet; $2-3$ residues at each end of this short linker sequence are needed to clear the van de Waals surfaces of $\mathrm{CaM}$ and the FMN-binding domain. This leaves 2-3 residues that are conformationally unrestricted, and hence, there are uncertainties about the exact position above the $\beta$-sheet of $\mathrm{CaM}$ and the orientation of the axis of the CaMrecognition site. The position of CaM relative to the FMN-binding domain is unspecified with respect to rotations about the y-axis of Fig. 3.8 by available information (corresponding to the axis of the CaM-binding helix of Fig. 3.8). Although the insertion is midway through the sequence of the FMN-binding module, in three dimensions the model predicts it to be directly adjacent to the CaM-binding site. The model also predicts that CaM-binding would be sterically hindered by the 
insertion, suggesting that the insertion can exist in more than one physiologically relevant conformation.

The structural model strongly suggests that the insertion can function as a control element. Firstly, the presence of the insertion correlates with $\mathrm{Ca}^{2+} / \mathrm{CaM}$ dependent regulation. Secondly, the close proximity of the insertion with the CaMbinding domain would likely engineer steric hindrance of CaM-binding. Hence, we proposed a role for the peptide insertion within the FMN-binding domain of cNOSs as an autoinhibitory control element (ACE).

\subsubsection{Synthetic Polypeptide Effects on Activity}

The possible functional significance of the putative ACE of cNOSs was evaluated using a series of synthetic polypeptide fragments corresponding to various regions of the ACE-derived sequence from nNOS and eNOS. If the insertion served an autoinhibitory function, peptides derived from this region might be expected to interfere with CaM-binding to, and activation of eNOS. Peptides were synthesized that correspond to various regions of the insertion ranging in length from 6 to 33 residues; specification of polypeptide sequence and origin is provided in Table 3.1. Five peptides corresponding to the regions flanking the insertion were also synthesized as controls. The effect of one of the insert-derived peptides ( $\operatorname{NOS}_{617-639}$ ) on the activity of nNOS, eNOS and iNOS is shown in Fig. 3.9. At concentrations of $50-100 \mu \mathrm{M}$ this peptide profoundly inhibits eNOS and nNOS activity, while iNOS is essentially unaffected. Several other insertion-derived peptides also elicited profound inhibition of eNOS and nNOS activity. Generally, the inhibition profiles for eNOS and nNOS were similar, although most peptides inhibited nNOS more potently than 

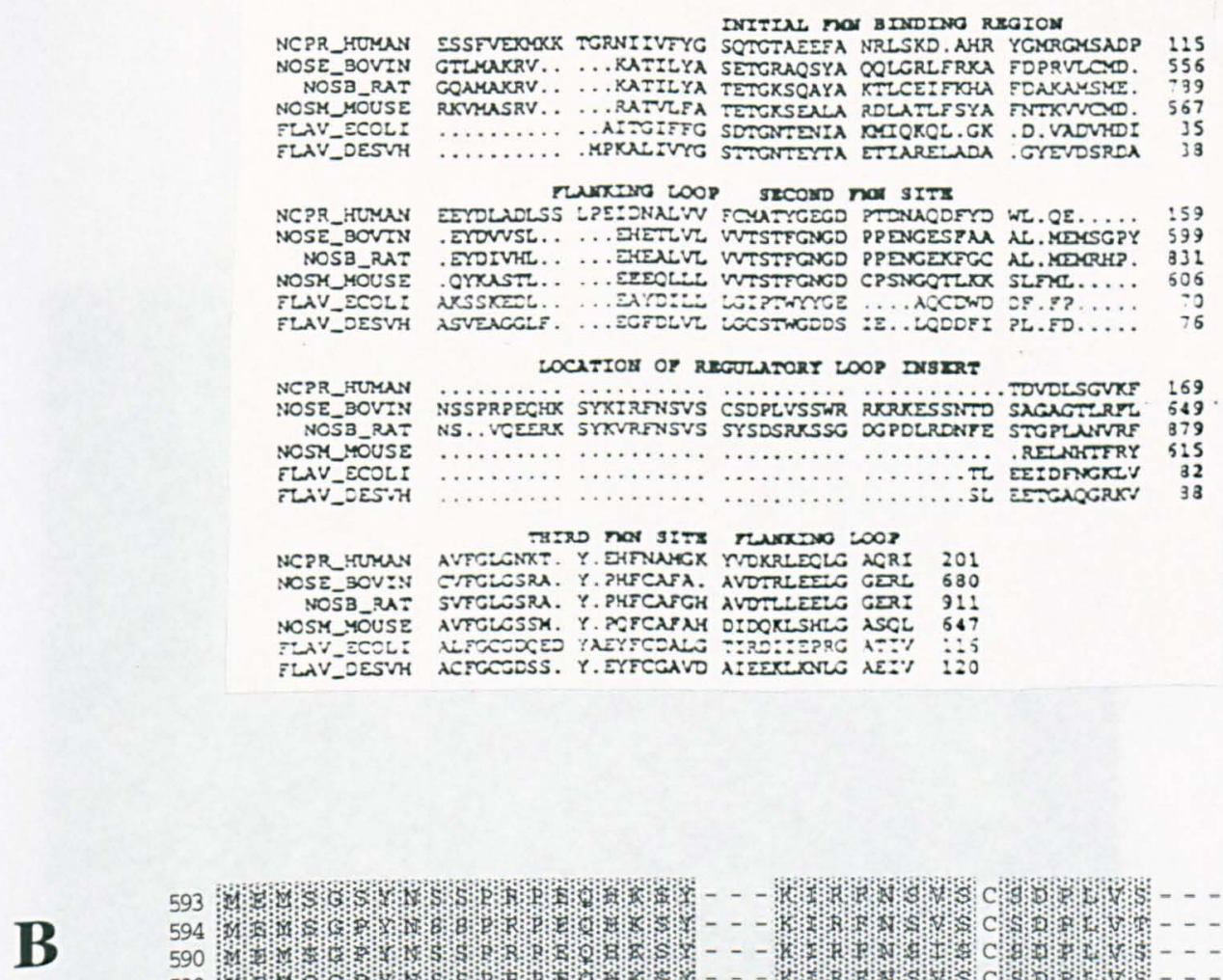

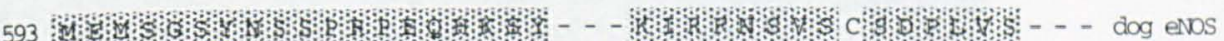

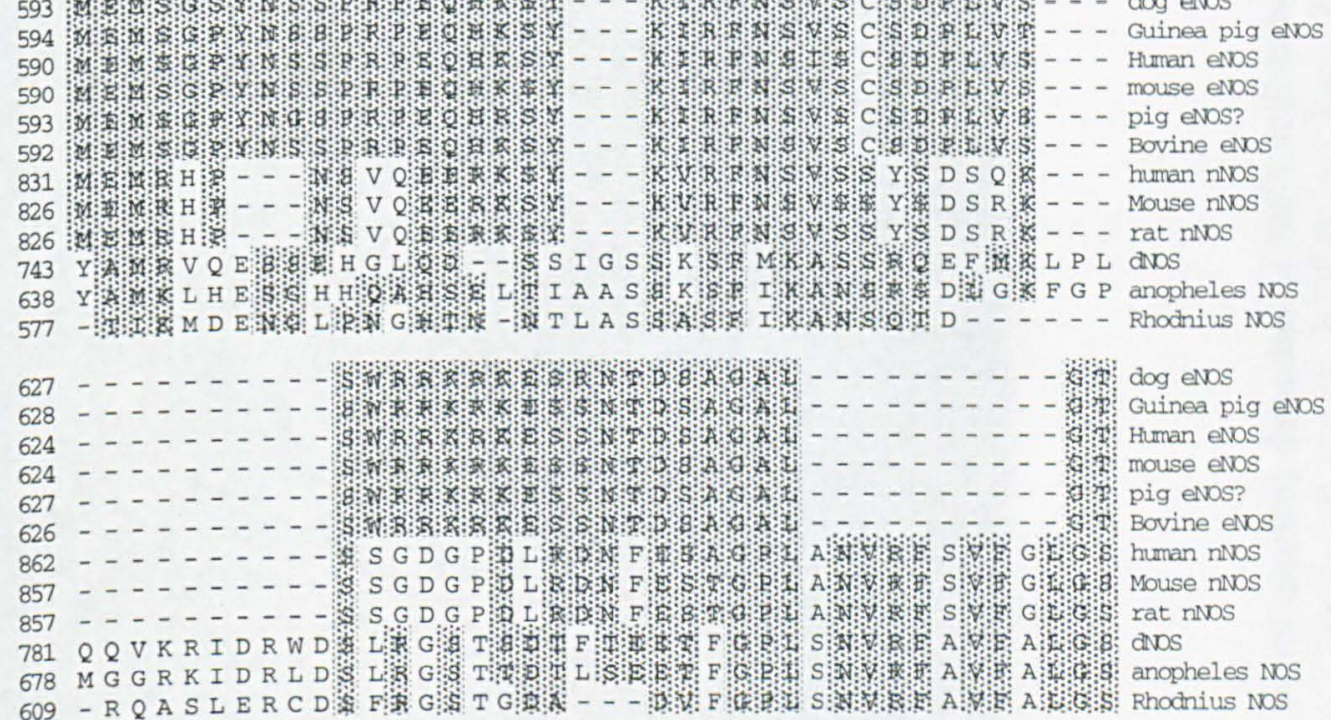

Fig. 3.6. Sequence alignment of NOS isoforms and related proteins showing location and conservation of the FMN-insertion. Panel A: Structure-based alignments of FMN binding domains of NOS isoforms with related bacterial flavoproteins showing location of the FMNinsertion peptide. Alignments were performed as described in Methods. Proteins represented are human $\mathrm{NADP}^{+}$-cytochrome $\mathrm{P} 450$ reductase (NCPR_HUMAN), bovine eNOS (NOSE BOVIN), rat nNOS (NOSB_RAT), murine iNOS (NOSM MOUSE) and flavodoxins from E.coli (FLAV_ECOLI) and $\bar{D}$. vulgaricus (FLAV_DES). Panel B: Homology based alignments of the FMN-insertion peptides of cNOSs from various species to show regions of conservation (shaded). Alignment was performed by the Clustal method using the DNA Star Megalign, a program in the Lasergene suite of molecular biology tools. 


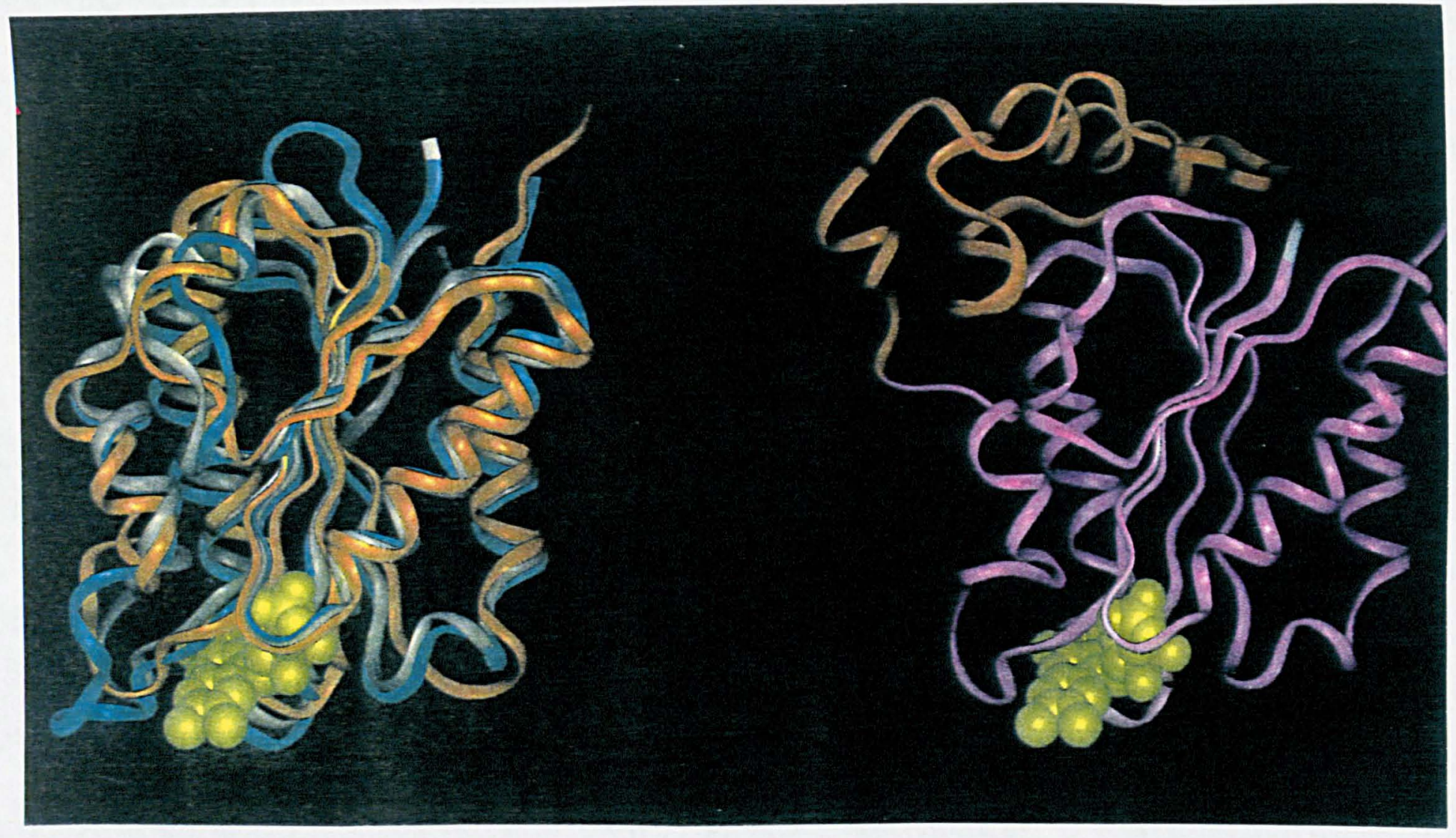

Fig. 3.7. Structural models of the FMN binding domains of NOS isoforms and related flavoproteins. Panel A (left): Bacterial flavodoxin (silver), CPR (gold) and rat iNOS (blue). Panel B (right): Bovine eNOS (pink) with the insertion peptide (gold) in an arbitrary conformation and bound-FMN in a space filling representation (yellow). N-terminal residues of the CaM binding site (white) are directly adjacent to the insertion peptide. Models were created as described in Methods using the Discover program (Biosym/Molecular Simulations). 


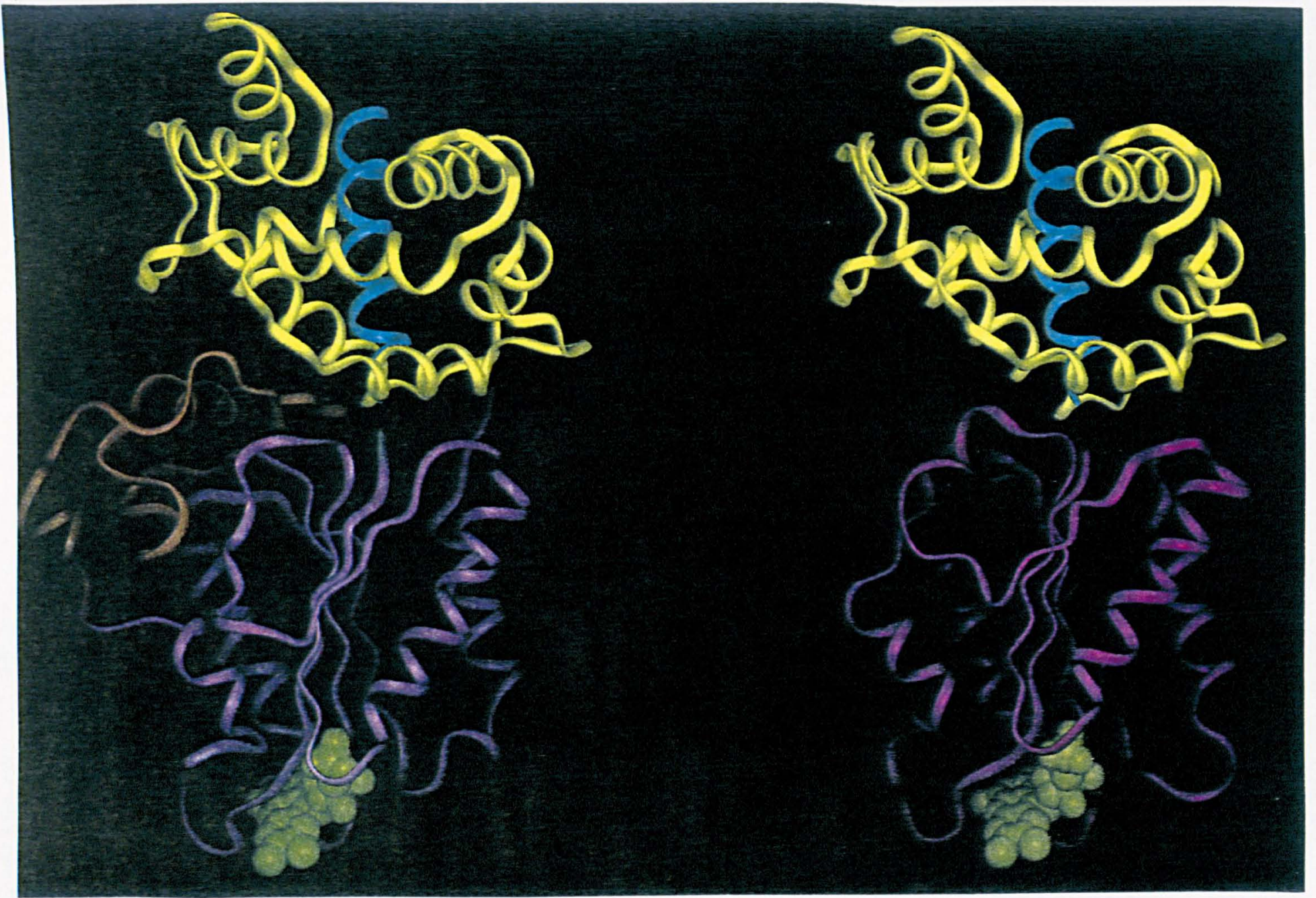

Fig. 3.8. Structural models of the FMN binding domains of eNOS and iNOS with bound-CaM. Panel A (left): eNOS FMN binding domain (pink) with the insertion peptide (gold). Panel B (right): iNOS FMN binding domain (purple). In bothe cases CaM (yellow) is shown bound to the CaM binding sequence (blue). Models were created as described in Methods using the Discover program (Biosym/Molecular Simulations). 
eNOS. However, inhibition was not observed with peptides derived from the regions of cNOS flanking the insertion. Effects of the various ACE-derived peptides are summarized in Table 3.2, the most effective polypeptides were derived from the eNOS insertion and contained an RRKRK motif. Peptides relating to the nNOS insertion weakly inhibited eNOS, but were without effect on nNOS.

Interestingly, even iNOS activity could be significantly inhibited by some of the cNOS insertion-derived peptides. Although all the peptides tested demonstrated a reduced efficacy for inhibition of iNOS, significant inhibition of iNOS was observed with $\mathrm{eNOS}_{601-633}$ and eNOS $607-633$. For example, eNOS $601-633$ inhibited iNOS activity with an $\mathrm{IC}_{50}$ of more than $1000 \mu \mathrm{g} / \mathrm{ml}$, while the observed $\mathrm{IC}_{50}$ for nNOS was only $31.0 \pm 1.1 \mu \mathrm{g} / \mathrm{ml}$. However, due to the extremely high affinity binding of CaM to iNOS (Cho et al., 1992), with little CaM dissociation occurring even after boiling in SDS, inhibition of iNOS is unlikely to involve the displacement of bound CaM. The observed inhibition of iNOS, may indicate the presence of vestigial "docking" sites for the insertion. Notably inhibition of iNOS activity by these peptides was rapid and apparently complete within one minute of addition (not shown).

\subsubsection{Synthetic Polypeptide Effects on CaM-binding}

The molecular model described in Fig. 3.8 suggests that the cNOS insertion overlaps the CaM-binding site to a degree, and that the insert may therefore impede CaM-binding. If this process involves docking of the insertion with cNOSs, peptide effects on CaM-binding to cNOSs may provide insights into the mechanism of inhibition of NOS activity described above. The effects of some representative peptides on CaM-binding to eNOS (panel A) and nNOS (panel B) are shown in Fig. 


\section{Effect of eNOS (617-639) on nNOS, iNOS and eNOS Activity}

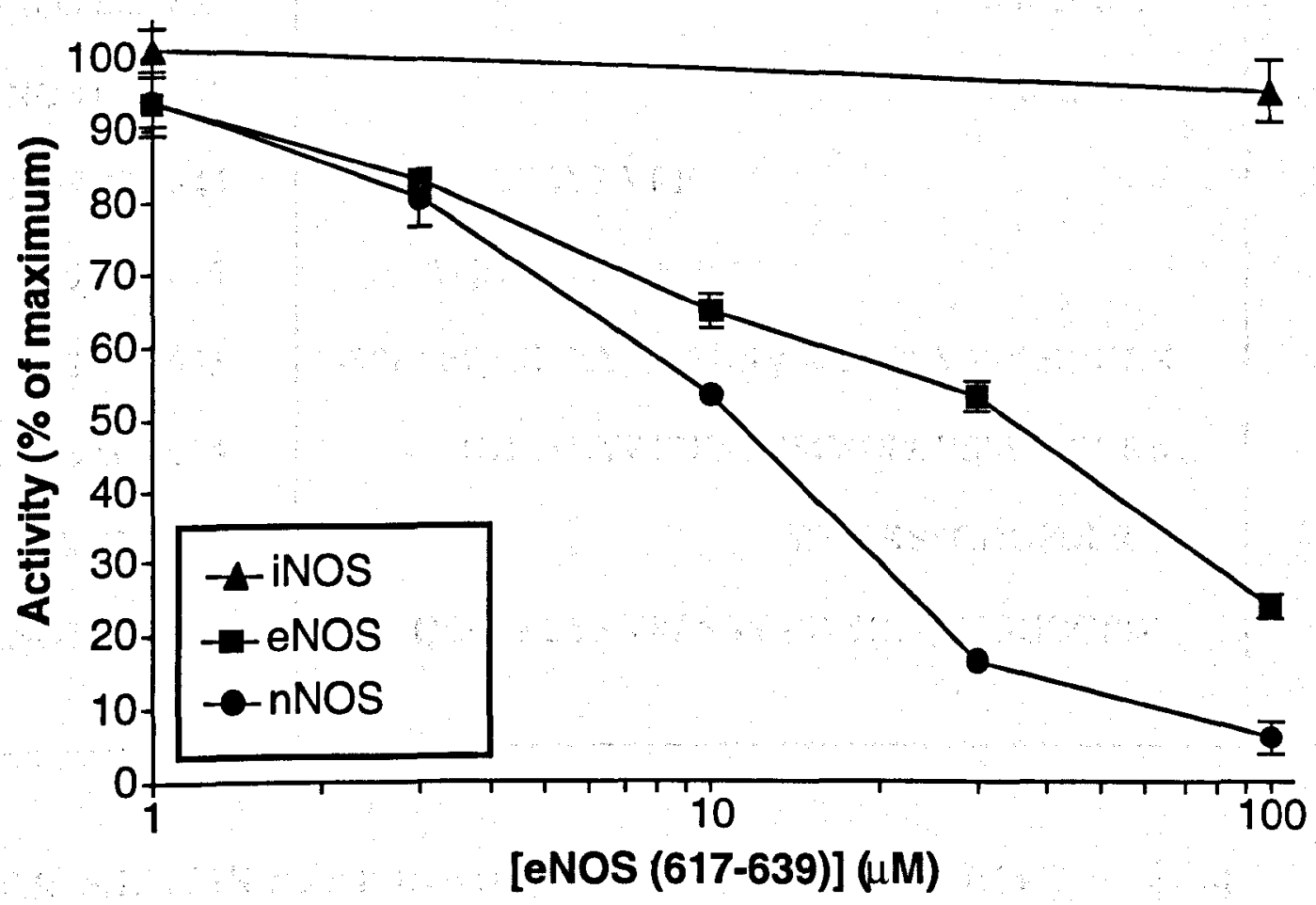

Fig. 3.9. Inhibitory effect of eNOS $617-639$ on the activity of the three NOS isoforms. The activity of each isoform (10 pmoles) was assessed using the Griess assay with $100 \mathrm{nM} \mathrm{CaM}$ and optimal levels of $\mathrm{Ca}^{2+}(100 \mu \mathrm{M})$. Activity is expressed as a percentage of the control activity in the absence of added peptide. Values shown are the mean of triplicates experiments \pm SEM. 


\begin{tabular}{|c|c|}
\hline Peptide Derivation & Sequence \\
\hline b eNOS 628-633 & WRRKRK \\
\hline b eNOS 626-636 & SSWRRKRKESS \\
\hline $\mathrm{h}$ nNOS $835-845$ & QEERKSYKVRF \\
\hline h eNOS 604-615 & RPEQHKSYKIRF \\
\hline b eNOS 601-633 & SSPRPEQHKSKYKIRFNSVSCSDPLVSSWRRKRK \\
\hline b eNOS 607-634 & QHKSKYKIRFNSVSCSDPLVSSWRRKRKE \\
\hline r nNOS $851-864$ & SDSRKSSGDGPDLR \\
\hline $\mathrm{h}$ nNOS $835-864$ & QEERKSYKVRFNSVSSYSDSQKSSGDGPDL \\
\hline
\end{tabular}

Table 3.1. FMN insert-derived peptide sequence and origin. Species of origin is given as human $(h)$, bovine $(b)$ or rat $(r)$. 


\begin{tabular}{|c|c|c|c|c|c|c|}
\hline $\begin{array}{c}\text { Peptide } \\
\text { Derivation }\end{array}$ & $\begin{array}{c}\text { Conc } \\
(\mu \mathrm{M})\end{array}$ & \multicolumn{2}{|c|}{ NOS Activity (\% of Control) } & \multicolumn{2}{c|}{ Ligand Binding to nNOS } \\
\cline { 4 - 7 } & & nNOS & eNOS & iNOS & ['25]-CaM & {$\left[{ }^{3} \mathrm{H}\right]-\mathrm{NNA}$} \\
\hline eNOS 628-633 & 107.0 & $11.0 \pm 3.3$ & $24.0 \pm 2.8$ & $91.1 \pm 5.7$ & $6.7 \pm 3.6$ & $91.7 \pm 3.6$ \\
eNOS 626-636 & 71.2 & $19.1 \pm 0.9$ & $27.7 \pm 2.0$ & $92.1 \pm 1.3$ & $0.0 \pm 0.5$ & $120.7 \pm 12.7$ \\
nNOS 835-845 & 68.1 & $102.0 \pm 2.1$ & $93.6 \pm 2.3$ & $101.1 \pm 3.7$ & $82.7 \pm 1.1$ & $118.9 \pm 4.1$ \\
eNOS 604-615 & 63.0 & $54.5 \pm 1.8$ & $80.5 \pm 2.0$ & $99.7 \pm 5.7$ & $24.7 \pm 2.2$ & $115 \pm 8.6$ \\
eNOS 601-633 & 76.0 & $30.4 \pm 1.7$ & $57.2 \pm 7.5$ & $62.2 \pm 2.8$ & $0.0 \pm 7.4$ & $116.3 \pm 1.9$ \\
eNOS 607-634 & 87.7 & $28.2 \pm 0.9$ & $40.2 \pm 4.0$ & $64.3 \pm 3.2$ & $0.0 \pm 4.8$ & $122.4 \pm 1.0$ \\
nNOS 851-864 & 72.0 & $98.4 \pm 1.9$ & $102.6 \pm 4.1$ & $99.7 \pm 2.8$ & $89.2 \pm 6.2$ & $119.2 \pm 3.4$ \\
nNOS 835-864 & 84.8 & $103.0 \pm 1.3$ & $80.2 \pm 3.8$ & $84.1 \pm 3.5$ & $82.7 \pm 1.1$ & $95.7 \pm 4.7$ \\
\hline
\end{tabular}

Table 3.2. Summary of the effects of FMN-insert derived peptides on NOS activity and ligand binding. NOS activity measurements were based on the rate of NADPH oxidation (for $\mathrm{nNOS}$ and $\mathrm{eNOS}$ ) or myoglobin oxidation (iNOS). $\left[{ }^{[25} \mathrm{I}\right]-\mathrm{CaM}$ and $\left[{ }^{3} \mathrm{H}\right]-\mathrm{N}^{w}$-nitro-arginine $\left.\left({ }^{3} \mathrm{H}\right]-\mathrm{NNA}\right)$ binding were assessed using microfiltration assays as in Methods. All values represent the mean of triplicate determinations \pm SEM. 
3.10. Potent inhibition of ${ }^{125} \mathrm{I}-\mathrm{CaM}$ binding to both eNOS and nNOS was observed with insert-derived peptides. The relative efficacy of these peptides for inhibition of CaM-binding mirrored that observed for inhibition of activity, and is summarized in Table 3.2. No inhibition was observed with peptides derived from the insertionflanking regions (not shown). For nNOS, $\mathrm{IC}_{50}$ values of eNOS-derived peptides ranged from 1-10 $\mu \mathrm{M}$, with potency increasing as the RRKRK motif was progressively lengthened to include up to 33 residues.

Conceivably, this inhibition could be due to sequestration of $\mathrm{Ca}^{2+}$ by the peptide, however, inhibition of CaM-binding by insert-derived peptides could not be overcome by the addition of excess $\mathrm{Ca}^{2+}$ up to $1 \mathrm{mM}$ (Fig. 3.11).

Inhibition of nNOS activity (Fig. 3.12) and CaM-binding (Fig. 3.13, panel B) by insert-derived peptides (in this case, NOS $_{607-634}$ ) was fully reversed by excess $\mathrm{CaM}$, indicative of a competitive mode of inhibition. This was supported by double reciprocal plots of the saturation $\left[{ }^{125} \mathrm{I}\right]-\mathrm{CaM}$ binding to nNOS (Fig. 3.13, panel B). The fitted lines cross at the Y-axis, suggesting the peptide reduces the affinity of CaM binding to nNOS, but does not reduce the available number of sites. This implies that competition for binding sites exists between the peptides and CaM. Since the insertion peptides have an excess of positively-charged residues, repulsion rather than interaction with the similarly positively-charged CaM-binding site is anticipated. It is more likely that $\mathrm{CaM}$ and the insertion peptide compete by domain overlap, as opposed to binding to a common site. Alternatively, using the same logic that discounts an interaction between the insertion-peptide and the CaM-binding domain, a 
direct interaction between the insertion-peptide and acidic (negatively charged) regions of $\mathrm{CaM}$ is a possibility.

To ensure that NOS is the actual target for eNOS-derived peptides, the level of CaM-binding to the actual peptides was assessed. Direct binding of $\left[{ }^{125} \mathrm{I}\right]-\mathrm{CaM}$ to peptide, quantified in the absence of NOS, was undetectable at concentrations that inhibited $>90 \%$ of CaM-binding to nNOS (Fig. 3.14). Additionally, peptides bound $\mathrm{CaM}$ in the absence or presence of $\mathrm{Ca}^{2+}$ (unlike the $\mathrm{Ca}^{2+}$-dependent $\mathrm{CaM}$ binding to cNOSs), suggesting that peptides bind to an inactivated form of CaM (Not shown) and only at concentrations higher than required for inhibition of cNOS activity. From Fig 3.13, it is obvious that the insert-derived peptides have the effect of reducing the apparent affinity of nNOS for CaM by competitive inhibition. We therefore investigated the effect of these peptides on the rate of dissociation of $\mathrm{CaM}$ from preformed CaM-NOS complexes. eNOS $_{607-634}$ caused a modest increase in the rate of dissociation of CaM-eNOS complexes (Fig. 3.15, panel A); this effect was much more evident for CaM-nNOS complexes (Fig. 3.15, panel B). The experimental design prevented the re-association of $\left[{ }^{125} \mathrm{I}\right]-\mathrm{CaM}-\mathrm{cNOS}$ complexes by the addition of a 3000-fold molar excess of unlabelled CaM. For synthetic peptides to displace CaM from its binding site on cNOS, it must, at least transiently, form a ternary complex with CaM-bound cNOS.

\subsubsection{Synthetic Polypeptide Effects on the Activity of Other CaM-dependent}

\section{Enzymes}

The above findings implicate a direct interaction between the peptide and cNOS, as the basis for competitive inhibition. However, due to the fact that peptide- 
dependent inhibition can be reversed by the addition of excess $\mathrm{CaM}$, it is conceivable that the peptides could interact with either the NOS or the CaM. In order to ensure that NOS is the actual binding target of eNOS-derived peptides, the effect of these peptides on the $\mathrm{Ca}^{2+} / \mathrm{CaM}$ dependent phosphatase calcineurin (protein phosphatase 2B, PP2B) was investigated. This proteins were selected due to its high-affinity $\left(K_{d} 1\right.$ $5 \mathrm{nM}$ ) (Imparl et al., 1995) and reversible CaM binding, similar to that of cNOSs (Sheta et al., 1994; Vorherr et al., 1993). As shown in Fig. 3.16, the same peptides that inhibited nNOS and PDE I did not exert any inhibitory effect on PP2B (Fig 3.16, panel A and B). The peptide-dependent inhibition of cNOSs must therefore be due to an interaction between the peptide and NOS itself. Indeed, as previously shown, at concentrations of peptide sufficient to cause $>90 \%$ inhibition of CaM-binding to nNOS, no direct binding of peptide to CaM was detectable (Fig. 3.14).

\subsubsection{Effect of Synthetic Polypeptides on Arginine Binding}

Most of the previously established inhibitors of NOS influence the binding of substrate in a manner which can be detected as a loss in sites or binding affinity for the arginine analog, $\left[{ }^{3} \mathrm{H}\right]-\mathrm{N}^{\mathrm{G}}$-nitro-arginine. Thus it is notable that the cNOS-derived peptides cause a slight increase, rather than decrease, in $\left[{ }^{3} \mathrm{H}\right]-\mathrm{N}^{\mathrm{G}}$-nitro-arginine binding (see Table 3.2). This implies that the peptide-dependent inhibition of NOS activity is not the result of any reduction in arginine binding and that the substrate binding site in the oxygenase domain remains functional when NOS-peptide complexes are formed. 

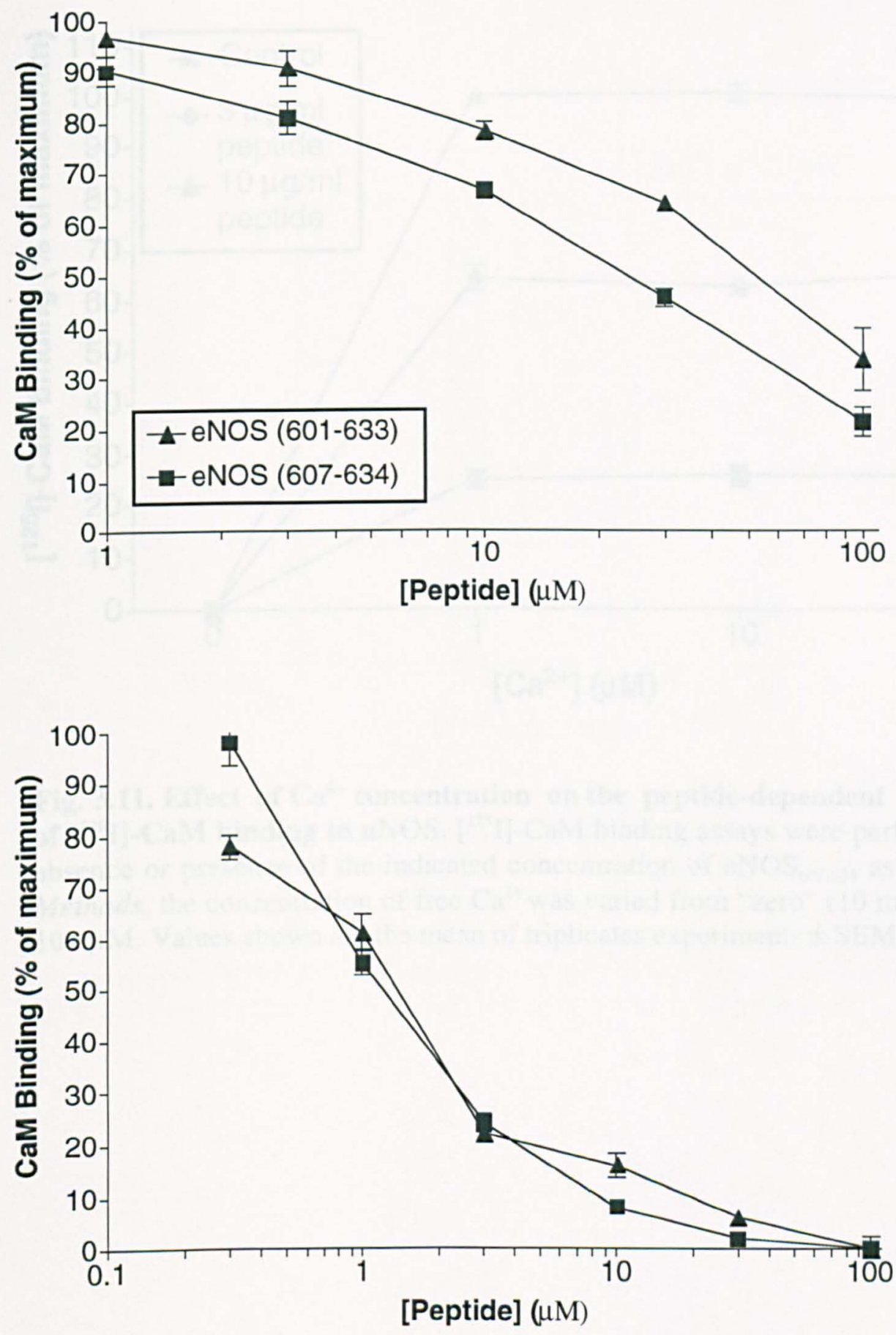

Fig. 3.10. Effect of eNOS $\mathrm{S}_{601-633}$ and $\mathrm{eNOS}_{607-634}$ on $\left[{ }^{125} \mathrm{I}\right]$-CaMbinding to eNOS and nNOS. Inhibition of $\left[{ }^{125} \mathrm{I}\right]-\mathrm{CaM}$ binding to eNOS (Panel A) and nNOS (Panel B) was assessed as a function of added peptide. $\left.{ }^{[25} \mathrm{I}\right]-\mathrm{CaM}$ binding was measured as described in Methods. Values are corrected for non-specific CaM binding and represent the mean of triplicate experiments \pm SEM. 


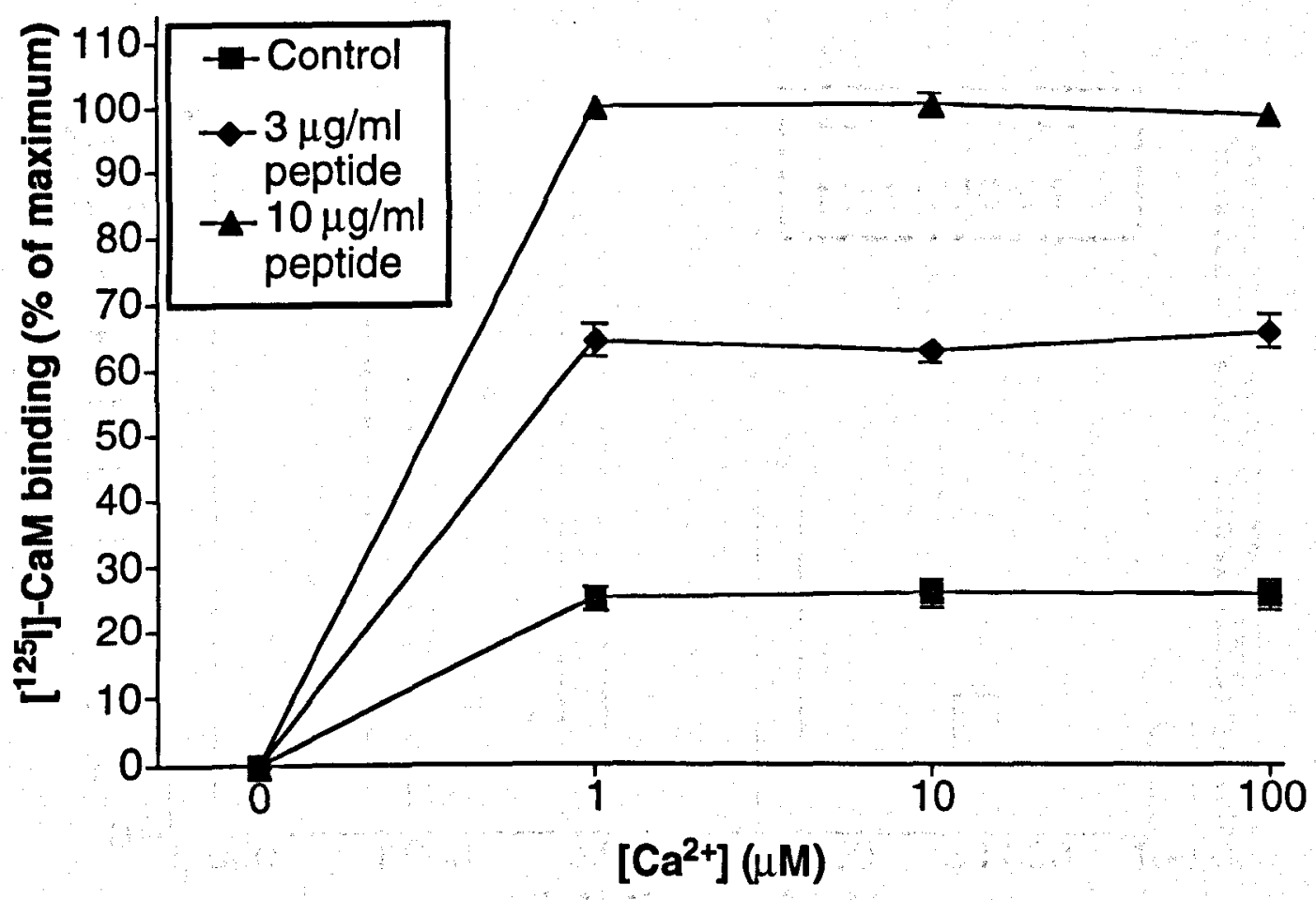

Fig. 3.11. Effect of $\mathrm{Ca}^{2+}$ concentration on the peptide-dependent inhibition of $\left.{ }^{125} \mathrm{I}\right]-\mathrm{CaM}$ binding to $\mathrm{nNOS}$. $\left.{ }^{125} \mathrm{I}\right]-\mathrm{CaM}$ binding assays were performed in the absence or presence of the indicated concentration of eNOS ${ }_{607-634}$ as described in Methods, the concentration of free $\mathrm{Ca}^{2+}$ was varied from "zero" (10 mM EGTA) to $100 \mu \mathrm{M}$. Values shown are the mean of triplicates experiments \pm SEM. 


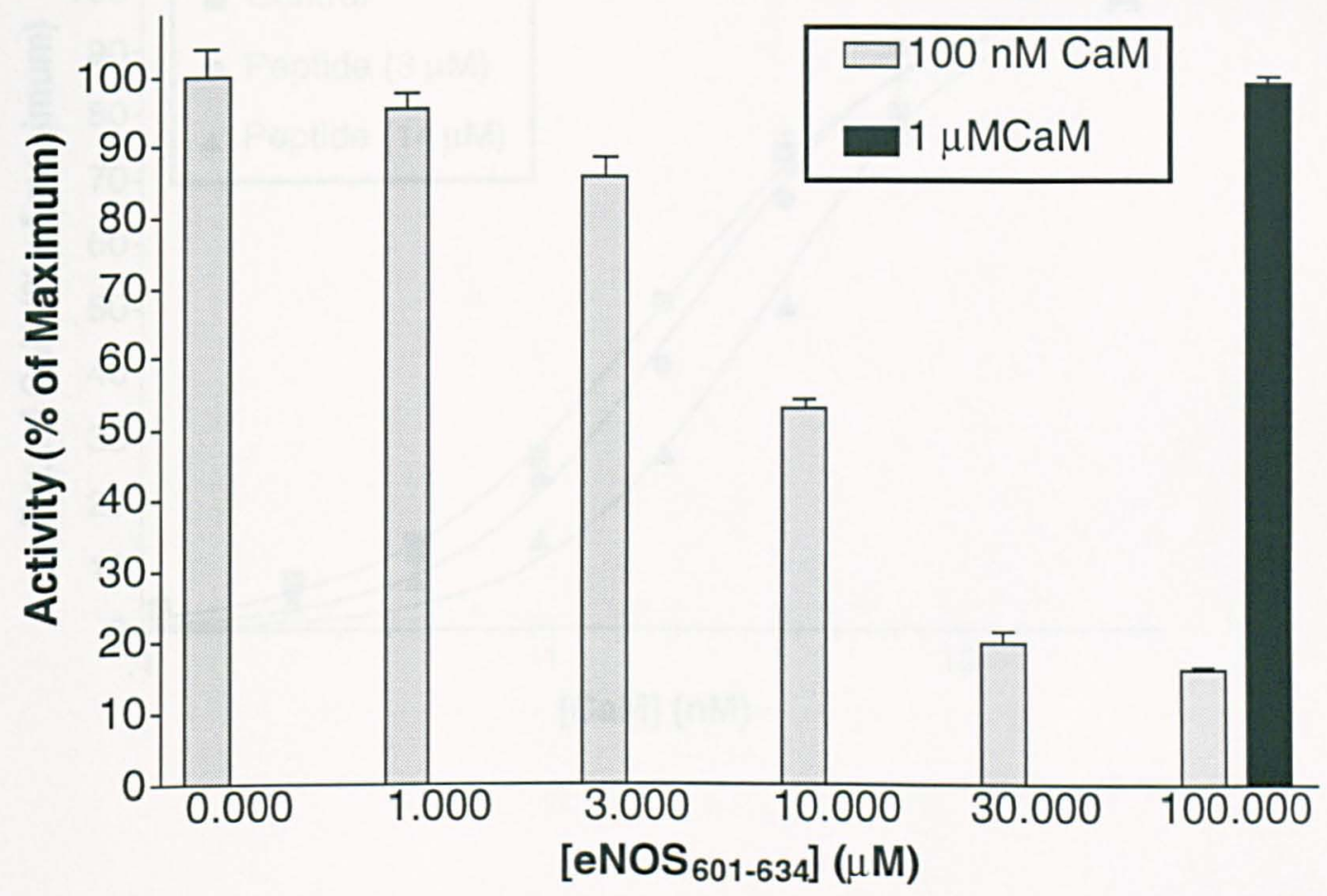

Fig. 3.12. Peptide-dependent inhibition of nNOS activity is fully reversible by the addition of excess calmodulin. The activity of nNOS (10 pmoles) was assessed kinetically based on the rate of NADPH oxidation and expressed as a percentage of the maximal rate in the absence of added peptide. The highest peptide concentration shows $>80 \%$ inhibition of nNOS activity at $100 \mathrm{nM} \mathrm{CaM}$, but activity is fully reversed by a 10 -fold increase in CaM concentration. 

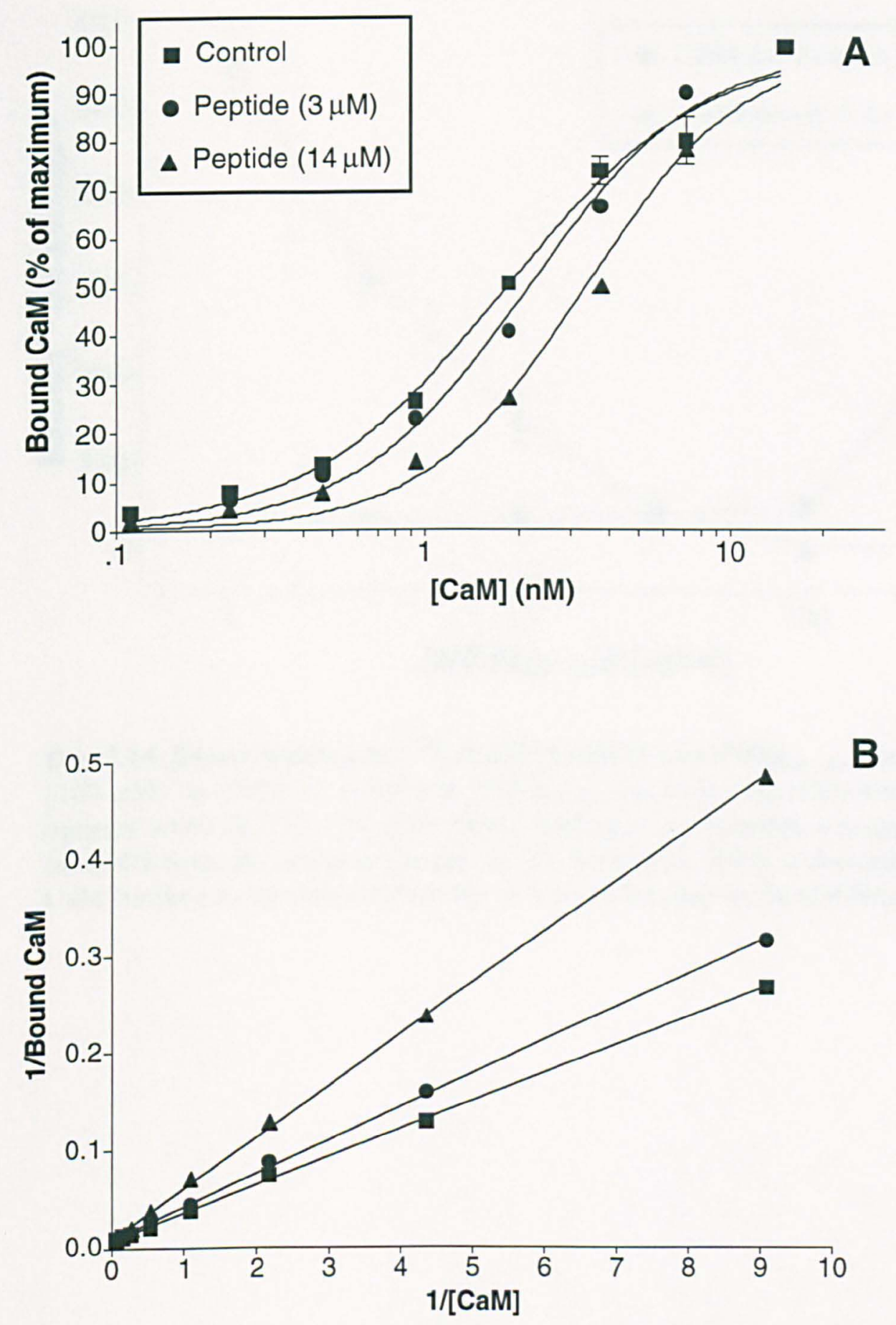

Fig. 3.13. Competitive inhibition of $\left[{ }^{125} \mathrm{I}\right]-\mathrm{CaM}$ binding to nNOS by insertion-derived peptide eNOS $_{607-634}$. Panel A: $\left[{ }^{125} \mathrm{I}\right]$-CaM binding assays were performed over a range of $\mathrm{CaM}$ concentrations, in the absence (control) or presence of added peptide. Panel B: The results of panel A are shown as a double reciprocal replot. Values are corrected for non-specific $\mathrm{CaM}$ binding and represent the mean of triplicate experiments \pm SEM. 


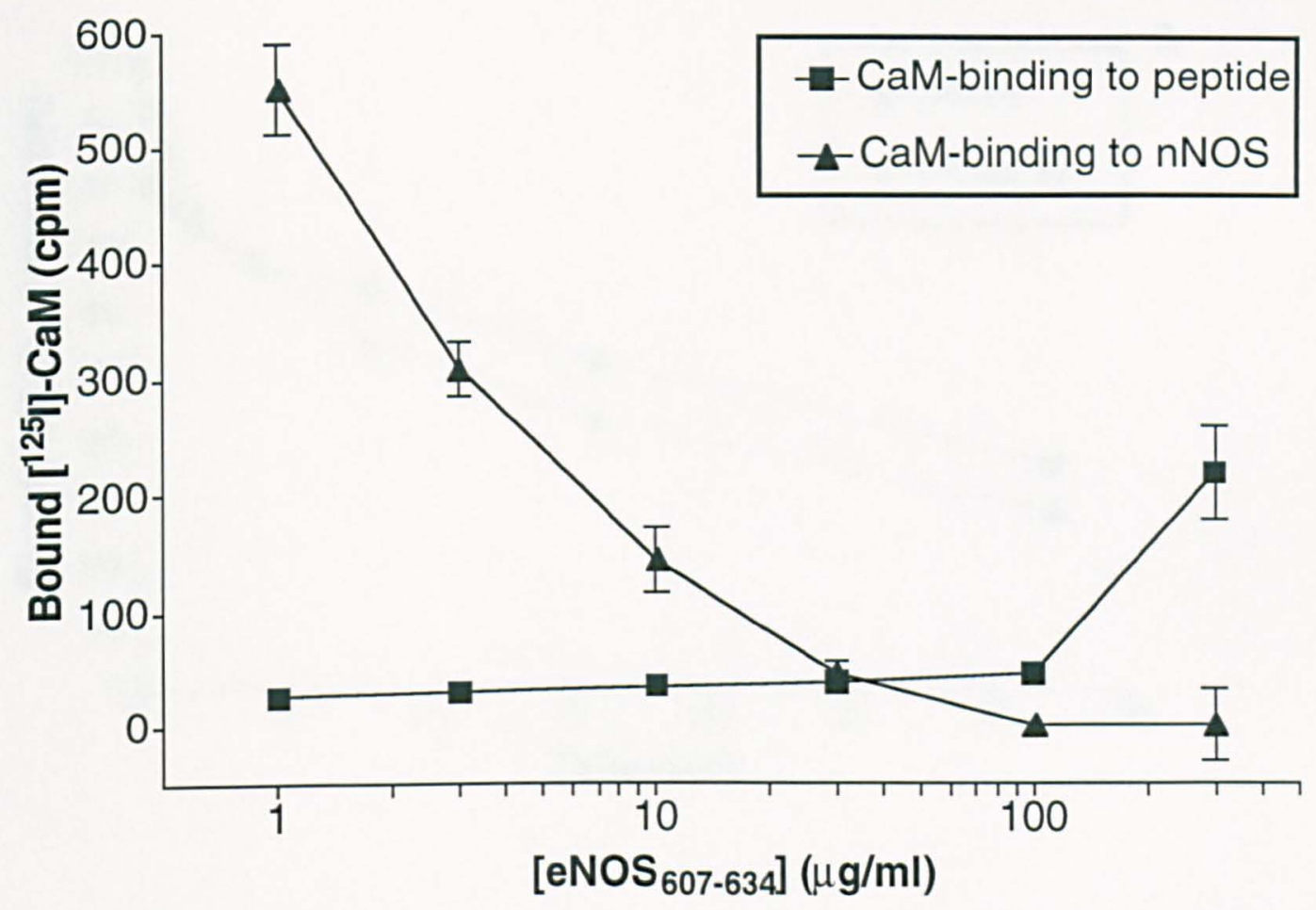

Fig. 3.14. Direct binding of $\left[{ }^{125} \mathrm{I}\right]-\mathrm{CaM}$ to $\mathrm{nNOS}$ and $\mathrm{eNOS}_{607-634}$. Binding of $\left[{ }^{125} \mathrm{I}\right]-\mathrm{CaM}$ $(100 \mathrm{nM})$ to $\mathrm{nNOS}(1 \mathrm{pmole})$ or $\mathrm{eNOS}_{607-634}$ (variable concentrations) was assessed at optimal levels of $\mathrm{Ca}^{2+}(100 \mu \mathrm{M})$. Direct binding to the peptides was assessed by omission of nNOS from the reaction mixture. Direct binding to nNOS is derived by subtracting the CaM-binding to the peptide from the total CaM-binding to the nNOS/peptide mixture. 

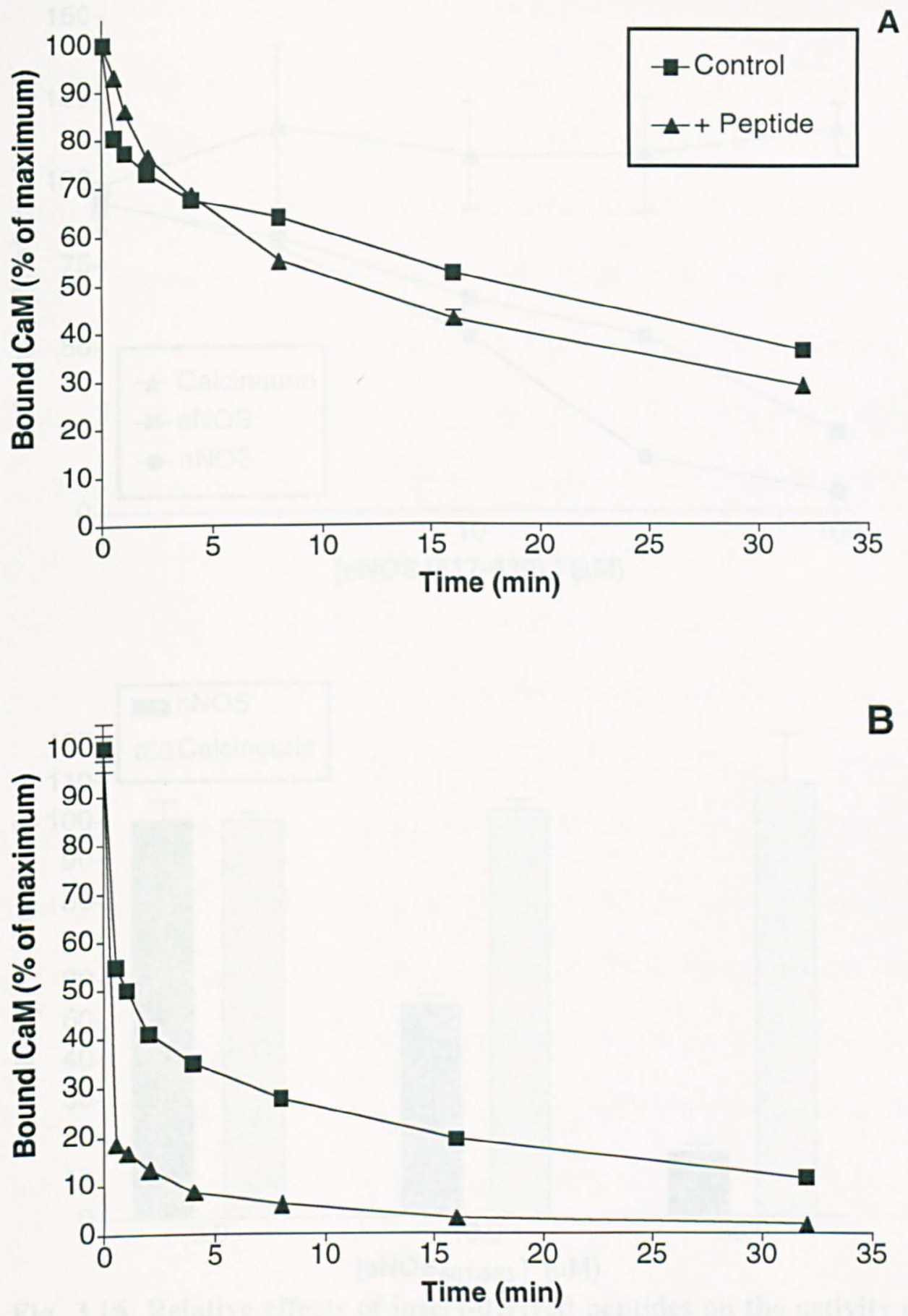

Fig. 3.15. Acceleration of $\left[{ }^{125} \mathrm{I}\right]-\mathrm{CaM}$ dissociation from cNOSs by eNOS $_{607-634} \cdot$ The time-dependent dissociation of eNOS-CaM (Panel A) or nNOS-CaM (Panel B) complexes in the absence (control) or presence (indicated concentration of peptide) was assessed as in Methods. Reassociation of labeled CaM was prevented by the addition of a 3000fold molar excess of unlabeled $\mathrm{CaM}$. Zero binding was defined as the amount in the presence of $10 \mathrm{mM}$ EGTA. Values shown are the mean of triplicate experiments \pm SEM. 

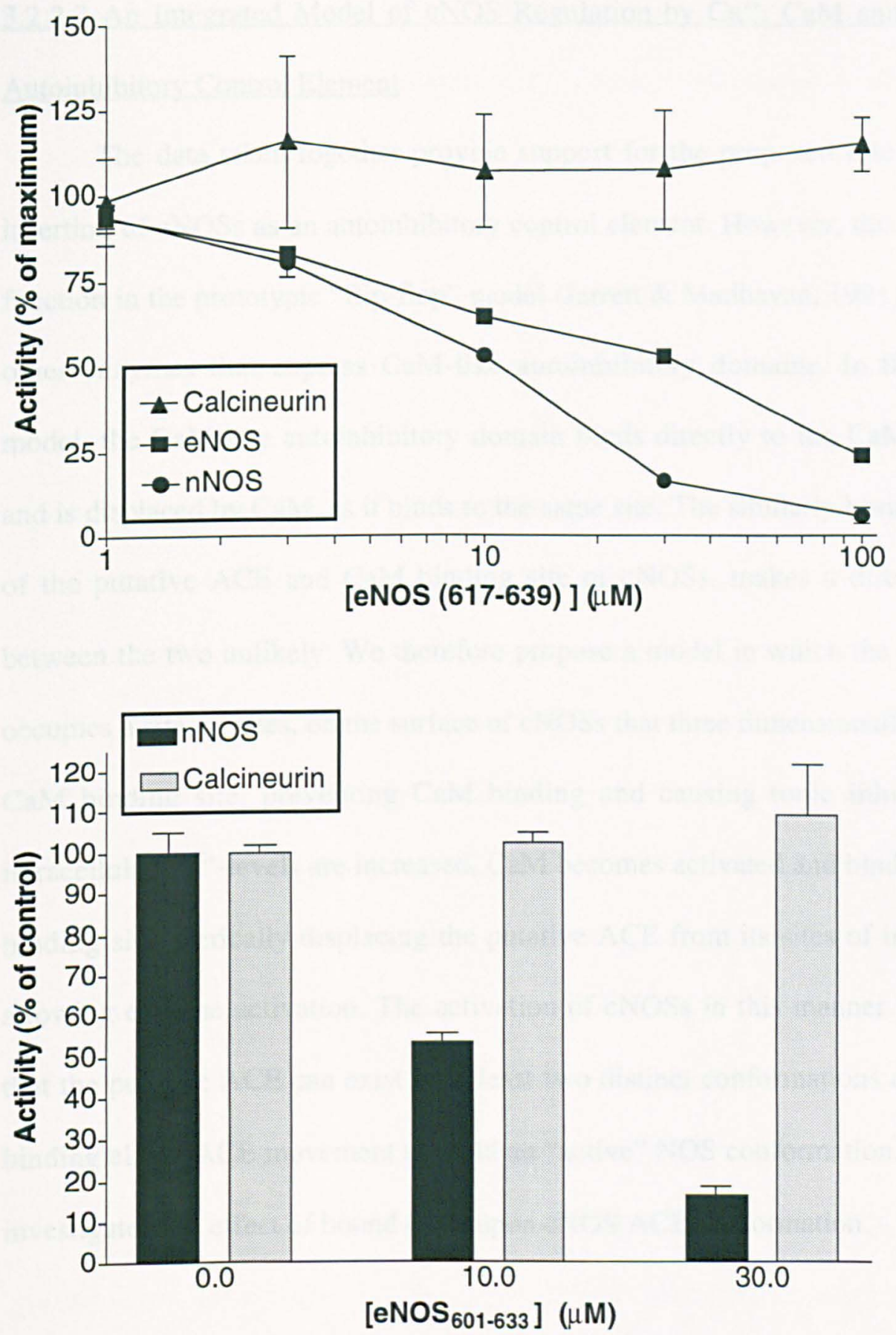

Fig. 3.16. Relative effects of insert-derived peptides on the activity of cNOSs and calcineurin. Panel A: Dose-dependent inhibition of nNOS, eNOS and calcineurin by $\mathrm{eNOS}_{617-639}$. Panel B: Relative potency of eNOS ${ }_{601-633}$ for inhibition of nNOS and calcineurin. Activity of cNOSs (10 pmole) was assessed by the Griess assay using $100 \mathrm{nM} \mathrm{CaM}$ at optimal concentrations of $\mathrm{Ca}^{2+}(100 \mu \mathrm{M})$. Calcineurin activity was assessed under the same conditions by a kinetic assay using the artificial substrate p-nitrophenyl phosphate. All activities are expressed as a percentage of the maximal activity observed in the absence of peptide. Values shown are the mean of triplicate experiments \pm SEM. 


\subsubsection{An Integrated Model of cNOS Regulation by $\mathrm{Ca}^{2+}, \mathrm{CaM}$ and the Putative}

\section{Autoinhibitory Control Element}

The data taken together provide support for the proposed role of the FMNinsertion of cNOSs as an autoinhibitory control element. However, this is unlikely to function in the prototypic "flip-flop" model (Jarrett \& Madhavan, 1991), accepted for other enzymes that express CaM-like autoinhibitory domains. In the "flip-flop" model, the CaM-like autoinhibitory domain binds directly to the CaM-binding site and is displaced by $\mathrm{CaM}$, as it binds to the same site. The similarly basic composition of the putative $\mathrm{ACE}$ and $\mathrm{CaM}$ binding site of cNOSs, makes a direct interaction between the two unlikely. We therefore propose a model in which the putative ACE occupies a site, or sites, on the surface of cNOSs that three dimensionally overlaps the $\mathrm{CaM}$ binding site, preventing $\mathrm{CaM}$ binding and causing tonic inhibition. When intracellular $\mathrm{Ca}^{2+}$-levels are increased, $\mathrm{CaM}$ becomes activated and binds to the $\mathrm{CaM}$ binding site, sterically displacing the putative ACE from its sites of interaction and allowing enzyme activation. The activation of cNOSs in this manner would predict that the putative ACE can exist in at least two distinct conformations and that CaMbinding elicits ACE movement to yield an "active" NOS conformation. We therefore investigated the effect of bound CaM upon cNOS ACE conformation.

\subsubsection{Effects of CaM on the Exposure of the Insert}

Limited proteolysis of proteins is one of the methods of choice to study conformational changes in proteins. A conformational shift in the protein structure changes the availability of proteolytic sites to digestion, resulting in a change in the pattern of observed cleavage fragments. Displacement of the insert from an internalized binding site on cNOSs by CaM-binding would conceivably enhance the 
exposure of the insert and increase its susceptibility to proteolysis. This hypothesis was tested by examining the pattern of peptide accumulation during limited trypsinolysis of both nNOS and eNOS, in the absence and presence of bound CaM (see Fig. 3.17 and 3.18).

It had previously been shown that of the possible 165 tryptic cleavage sites in rat nNOS, a single preferred cut site is present at $\mathrm{Arg}^{727}$ within the CaM-binding region (Sheta et al., 1994). Trypsinolysis of nNOS (see Fig. 3.17, panel A) in the absence of bound CaM (due to the chelation of $\mathrm{Ca}^{2+}$ ), results in the formation of fragments consistent with trypsinolysis occurring almost exclusively at this residue $\left(\operatorname{Arg}^{227}\right)$. Accordingly, we found a time-dependent accumulation of fragments with apparent molecular masses of 77 and $85 \mathrm{kDa}$, corresponding to the reductase and oxygenase domains, respectively. When nNOS-CaM complexes were permitted to form (no chelator present) trypsinolysis yielded fragments of apparent molecular masses of 63 and $93 \mathrm{kDa}$, indicating trypsinolysis at a new site. An accurate assessment of these masses was established by matrix-assisted laser desorption ionization mass spectrometry (MALDI-MS). The mass of the smaller fragment was determined at $64809 \pm 324 \mathrm{Da}$, best explained by cleavage at $\mathrm{Arg}^{855}$ within the insert (predicted to yield a C-terminal fragment of $65071 \mathrm{Da}$ ). Further confirmation that this fragment is derived from a cut site within the reductase domain is provided by trypsinolysis of bacterially-expressed reductase $\left(\mathrm{nNOS}^{721-1429}\right.$ ) and oxygenase domains $\left(\mathrm{nNOS}^{1.721}\right)$, the 65071 fragment can only be derived from trypsinolysis of the individually expressed reductase domain (Data not shown). Confirmation of this cleavage site as $\mathrm{Arg}^{855}$ is provided by $\mathrm{N}$-terminal sequencing of this fragment, the KSSGDGPDLR sequence obtained could only have resulted from trypsinolysis at this 
site. A cartoon depicting the relative positions of the various trypsinolysis sites is shown in Fig. 3.17 (panel B).

Similar studies using eNOS protein resulted in a slightly more complex (see Fig. 3.18, panel A), but analogous pattern of trypsinolysis. Trypsinolysis of CaM-free NOS resulted in four principal fragments of approximate molecular masses of 57, 60, 68 , and $77 \mathrm{kDa}$. This pattern is rationalized by cleavage at $\mathrm{Arg}^{518}$ within the CaMbinding region $\left(\mathrm{eNOS}^{1-518}=56877 \mathrm{Da}, \mathrm{eNOS}^{519.1204}=76308 \mathrm{Da}\right)$ and at a second site, likely to be $\mathrm{Lys}^{545}$ within the insert peptide $\left(\mathrm{eNOS}^{1.545}=59916 \mathrm{Da}, \mathrm{eNOS}^{546-1204}=\right.$ $73270 \mathrm{Da}$ ). Binding of $\mathrm{CaM}$ simplifies this cleavage pattern by providing a single dominant cut site, only two strong fragment bands are revealed by gel electrophoresis, at 60 and $65 \mathrm{kDa}$. These are predicted by cleavage of eNOS within the RRKRK motif of the insert peptide at Lys ${ }^{632}$ (with additional cleavage of the $\mathrm{N}$-terminal fragment at $\mathrm{Lys}^{545}$ ) producing fragments of $59916 \mathrm{Da}$ and $63251 \mathrm{Da}$. As seen in Fig. 3.6, alignment of the peptide inserts of eNOS and nNOS reveals a close correspondence between Lys $^{632}$ of eNOS and Lys ${ }^{856}$ of nNOS, suggesting a similar, CaM-driven movement of the peptide insertion in both cNOS isoforms. A cartoon representation of eNOS trypsinolysis sites is shown in Fig 3.18 (panel B).

\subsubsection{Function of the Conserved Regions of the Autoinhibitory Control Element}

In order to investigate the roles of the various regions of the autoinhibitory control element of cNOS, an attempt was made to identify areas of conservation. These conserved regions may represent sites required for interaction with a putative docking site that confers autoinhibitory function. 


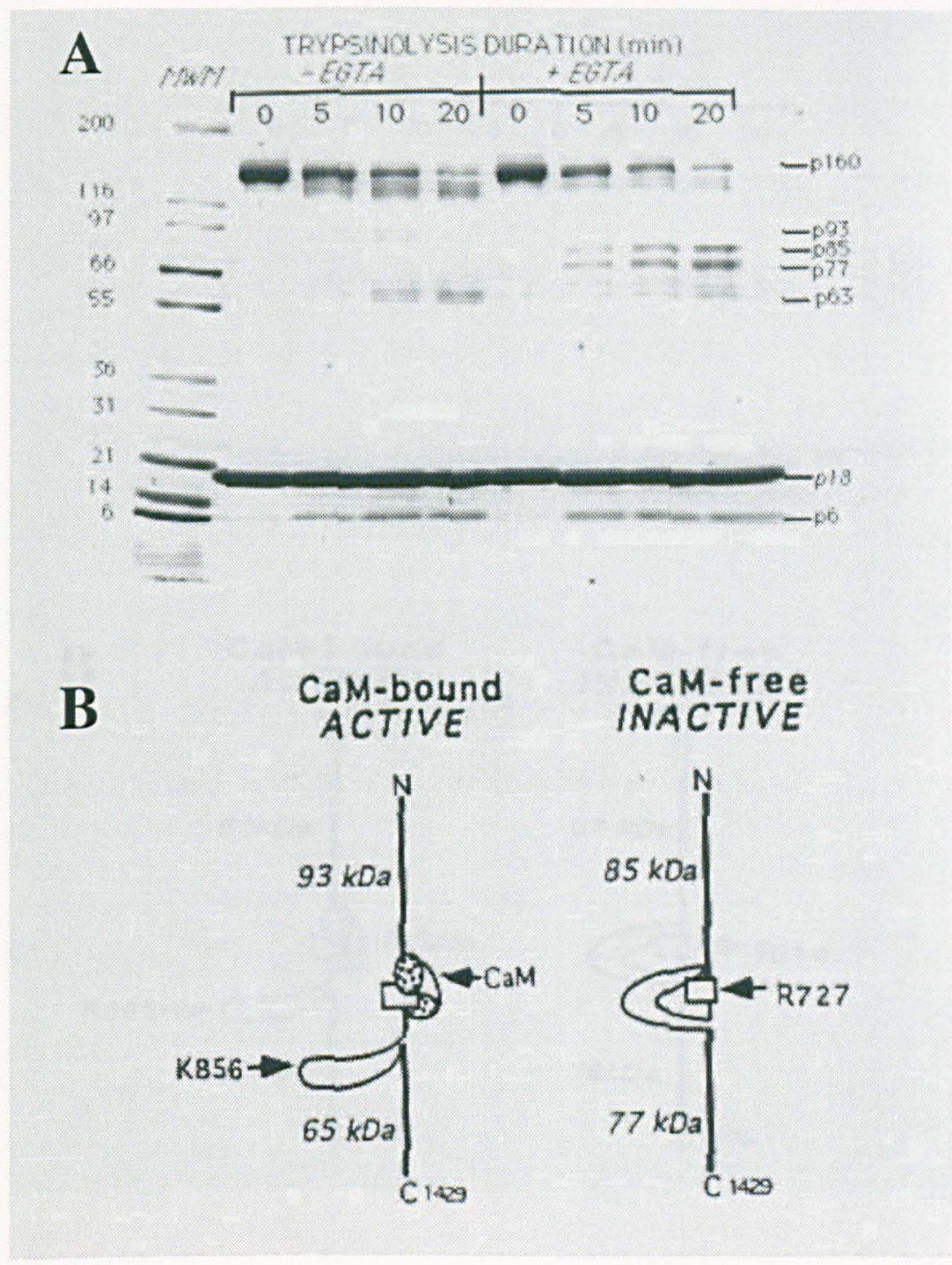

Fig. 3.17. SDS-polyacrylamide gel electrophoresis showing the products of limited tryptic digestion of nNOS. Panel A: Trypsinolysis of nNOS was performed for the indicated duration in the absence (+EDTA) or presence (-EDTA) of bound $\mathrm{CaM}$. Protein bands at 160 and $17 \mathrm{kDa}$ correspond to uncut nNOS and $\mathrm{CaM}$, respectively. Results indicate that a preferred tryptic cleavage site resides within the $\mathrm{CaM}$ binding sequence of nNOS, however, when this site is masked by bound $\mathrm{CaM}$, a novel tryptic cleavage site is revealed within the FMNinsertion peptide. Panel B: A representative cartoon of the tryptic cleavage sites of CaM-bound and CaM-free nNOS. 


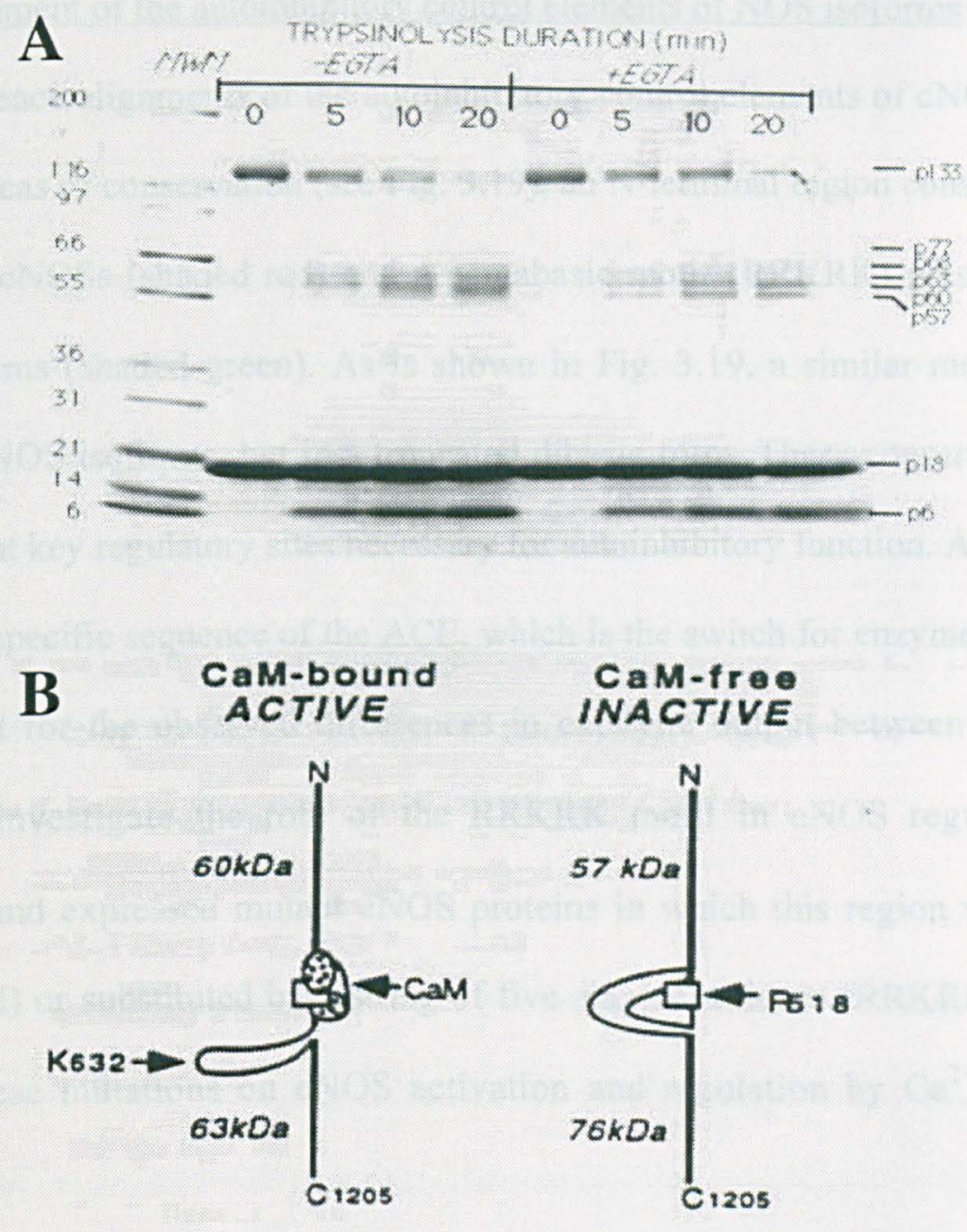

Fig. 3.18. SDS-polyacrylamide gel electrophoresis showing the products of limited tryptic digestion of eNOS. Panel A: Trypsinolysis of eNOS was performed for the indicated duration in the absence (+EDTA) or presence (-EDTA) of bound $\mathrm{CaM}$. Protein bands at 133 and $17 \mathrm{kDa}$ correspond to uncut $\mathrm{nNOS}$ and $\mathrm{CaM}$, respectively. Results indicate that a preferred tryptic cleavage site resides within the CaM binding sequence of eNOS, however, when this site is masked by bound $\mathrm{CaM}$, a novel tryptic cleavage site is revealed within the FMNinsertion peptide. Panel B: A representative cartoon of the tryptic cleavage sites of CaM-bound and CaM-free eNOS. 


\subsubsection{Alignment of the autoinhibitory control elements of NOS isoforms}

Sequence alignments of the autoinhibitory control elements of cNOSs reveals two major areas of conservation (see Fig. 3.19), an $\mathrm{N}$-terminal region conserved in all mammalian cNOSs (shaded red) and a pentabasic motif (RRKRK) present only in eNOS isoforms (shaded green). As is shown in Fig. 3.19, a similar motif may be present in nNOS isoforms, but in a truncated dibasic form. These conserved regions may represent key regulatory sites necessary for autoinhibitory function. Additionally, the isoform specific sequence of the ACE, which is the switch for enzyme activation, may account for the observed differences in catalytic output between eNOS and nNOS. To investigate the role of the RRKRK motif in eNOS regulation, we engineered and expressed mutant eNOS proteins in which this region was deleted (RRKRK del) or substituted by a string of five alanine residues (RRKRK sub). The effect of these mutations on eNOS activation and regulation by $\mathrm{Ca}^{2+} / \mathrm{CaM}$ was assessed.

\subsubsection{Expression and purification}

A two-step purification involving sequential affinity chromatographies on ADP-sepharose and CaM-sepharose resins resulted in $>90 \%$ homogeneity for wildtype nNOS, wildtype eNOS, RRKRK deletion and RRKRK substitution, as estimated from Coomasie-stained SDS/PAGE (Fig. 3.20). Low temperature SDS-gel electrophoresis analysis showed that the mutations did not attenuate NOS homodimer stability (Fig. 3.21). Overall protein yields ranged from $2-10 \mathrm{mg}$ purified protein per liter of bacterial culture. Spectrophotometry demonstrated that the CO-bound spectra of dithionite-reduced mutant and wt eNOS were indistinguishable, indicating that RRKRK deletion/substitution does not compromise heme-coordination (Fig. 3.22, 
panels A, B and C). Accordingly, spectral assessment of heme-chromophore was used to quantify eNOS protein mass.

\subsubsection{Catalytic Activities}

Three catalytic activities of eNOS were analyzed and contrasted for wildtype and RRKRK mutant enzymes (Fig. 3.23 and 3.24). These distinct activities were determined in the presence or absence of excess $\mathrm{Ca}^{2+}$ and indicate maximal electron flux to heme (NO synthesis, 3.23), FAD (ferricyanide reduction, Fig. 3.24, panel A) and FMN (cytochrome c reduction, Fig 3.24, panel B), in the absence and presence of bound-CaM (Adak et al., 1999).

As shown in Fig 3.23, neither wt nor mutant eNOS supported detectable levels of NO synthesis in the absence of added CaM. Deletion or substitution of the RRKRK motif resulted in a 2-3-fold increase in maximal CaM-induced NO synthesis, compared to wildtype enzyme (from $136 \pm 16$ to $450 \pm 17$ and $504 \pm 11 \mathrm{nmol} / \mathrm{min} / \mathrm{mg}$, respectively). Given that the rate-limiting step for NO synthesis by CaM-bound eNOS appears to reside in the reductase domain (Nishida \& Ortiz de Montellano, 1999; Nishida \& Ortiz de Montellano, 1998), we monitored the rate that artificial electron acceptors could be reduced by electrons derived from reductase domain flavins. Although significant basal cytochrome $\mathrm{c}$ and ferricyanide reduction was observed with both mutant and wildtype eNOS; basal activity was 2-3 fold greater for RRKRK del compared to wildtype eNOS (Figs. 3.24, panels A and B). In the case of wildtype and RRKRK del eNOS, addition of CaM caused a further 2-3 fold increase in the rate of reduction of these electron acceptors. RRKRK sub eNOS exhibited a much higher basal rate than either wildtype or RRKRK del, the maximal rate in the 
presence of $\mathrm{CaM}$ was, however, comparable to that of the deletion. For both cytochrome $c$ and ferricyanide reduction, the CaM-free basal activity of RRKRK mutants was comparable to, or higher than, the CaM-bound activities of wildtype eNOS. This implies that the reductase function of CaM-bound eNOS is attenuated, and that the RRKRK motif is responsible for this attenuation. Hence, it seems that the presence of the RRKRK motif in wildtype eNOS causes attenuation of the otherwise faster rate of electron flow from NADPH to FAD, and from FAD to FMN. Removal of this motif results in an increase in both the CaM-independent and -dependent rates of reduction. However, CaM-binding is still required for NO synthesis, presumably inducing conformational changes, which align the FMN and heme moieties, required for electron transfer to the oxygenase domain.

\subsubsection{4 $\mathrm{Ca}^{2+}$-dependenceof Calmodulin Binding and Activation}

Given the putative role of the autoinhibitory control element in controlling CNOS activation by $\mathrm{Ca}^{2+} / \mathrm{CaM}$, we investigated the role of the RRKRK motif in the control of $\mathrm{Ca}^{2+}$-dependent functions of the enzyme. Experiments were performed to compare the $\mathrm{Ca}^{2+}$-dependence for activation of wildtype, RRKRK del, and RRKRK sub eNOSs ( $80 \mathrm{nM})$ by CaM $(100 \mathrm{nM})$. As shown in Fig. 3.25 (panel A), removal of the RRKRK motif , by deletion or substitution, did not significantly diminish the $\mathrm{Ca}^{2+}$. dependence for activation of eNOS by $\mathrm{Ca}^{2+} / \mathrm{CaM}$. On the contrary, a slight increase in the levels of $\mathrm{Ca}^{2+}$ required for activation was observed with both the substitution and deletion mutants.

We next compared the $\mathrm{Ca}^{2+}$-dependence for $\left[{ }^{125} \mathrm{I}\right]-\mathrm{CaM}$ binding to RRKRK mutants and wildtype eNOS. Assessment of both $\left[{ }^{125} \mathrm{I}\right]$-CaM binding (Fig. 3.25, panel 
585 E S F A A A L MEMSGPYN S S PRPEQHKS YK IR F N S S C S D P Bovine eNOS

586 E S F A A A L MEMSGSYNSSPRPEQHKSYKIRFNSVS CSDP dog ENOS

587 E S F A A A L M E M S GPYN S S PRPEQHKS Y K IR F N S V S C S D P Guinea pig eNOS

583 E S F A A A L M M S G P Y S S PRPEQHKSYKIR FNSIS C S P Human eNOS

583 E S F A A A L M M S GPYNSSPRPEQHKSYKIR FN SVS C S D P mouse eNOS

824 EKF G C A L MEMR- - HPN S Q E ERKSYKVRFNSVS S Y S D human nNOS

819 EKF G C A L MEMR- - HPN SVQEERKS Y KVRFN SVS S Y S D Mouse nNOS

819 EKF GCALMEMR-- - HPNSVQEERKSYKVRFNSVSSYS D rat nNOS

$1 \ldots \ldots$.

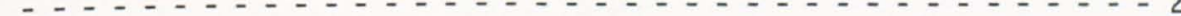

623 LVS SWRRKRKESSNTDSAGALGTLRF CVFGLGSRAYPH Bovine eNOS

624 LVS SWRRKRKESR NTDSA G A L GTLRFCVFGLGSRAYPH dog eNOS

625 L V T S WR R KR K E S S N T D S A G A L G T LR F C V F G L G SR A Y P H Guinea pig eNOS

621 LVS SWRRKRKESSNTDSAGALGTLRFCVFGLGSRAYPH Human eNOS

621 LVS S WRRKR.KESSNT DS A GALGTLRFCVFGLGSRAY P H mouse eNOS

859 S Q K S S GDGPDLR DNFESA GPLANVRFS VF GL G SRAY P H human nNOS

854 SRKSSGDGPDLRDNFESTGPLANVRFS VFGLGSRAYPH Mouse nNOS

854 SRKSS GDGPDLRDNFESTGPLANVRFS VFGLGSRAYPH rat nNOS

13

1

$\frac{1}{2}$

Fig. 3.19. Regions of conservation within the FMN-insertions of eNOS and nNOS. Alignment of the FMN-insertion regions of representative members of the nNOS and eNOS families of proteins. Special note should be made of the RRKRK motif (shaded green), perfectly conserved in eNOS proteins, but absent from nNOS. Also a region near the $\mathrm{N}$-terminal end of the insertion (shaded red), which is conserved throughout the $\mathrm{Ca}^{2+}$-dependent isoforms. 
Del Sub

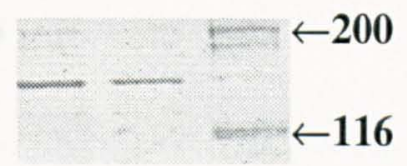

Fig. 3.20. SDS-polyacrylamide gel electrophoresis of purified recombinant RRKRK del and RRKRK sub eNOS. Recombinant NOS proteins were all purified to $>90 \%$ homogeneity and showed approximate molecular weights of 135 $\mathrm{kDa}$ for both eNOS mutants. 


\section{Del Sub \\ $200 \rightarrow$ \\ $130 \rightarrow$}

Fig. 3.21. Stability of RRKRK mutant eNOS homodimers by lowtemperature SDS-polyacrylamide gel electrophoresis. Low temp SDSPAGE was performed as described in Methods. The apparent molecular weight of $>200 \mathrm{kDa}$ indicated the formation of stable NOS homodimers for both eNOS mutants. 

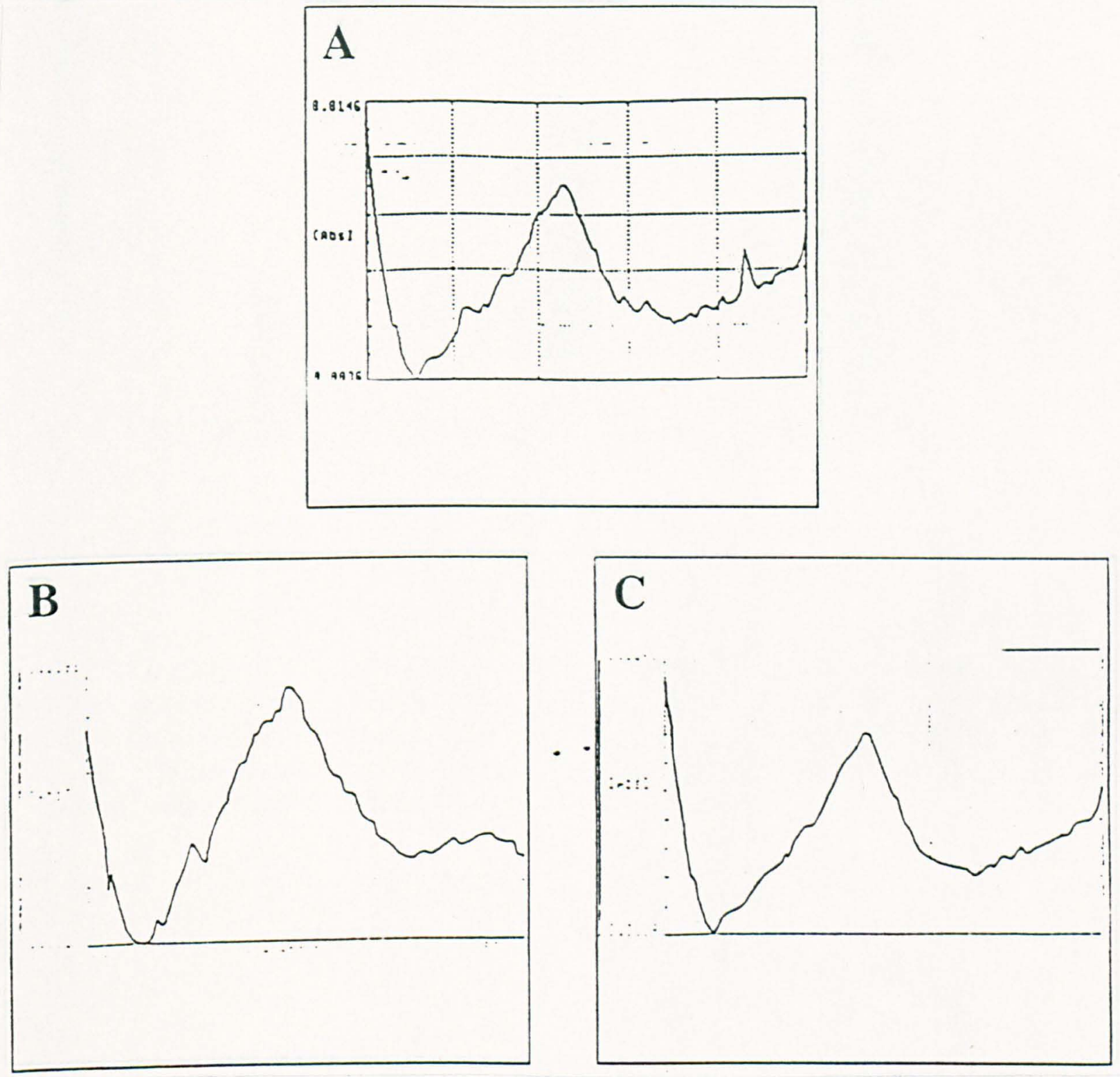

Fig. 3.22. P450 spectra of wildtype, RRKRK del and RRKRK sub eNOS. The CO-bound, dithionite reduced spectra of wild type (panel A), RRKRK del (panel B) and RRKRK sub (panel C) were obtained as described in methods. 


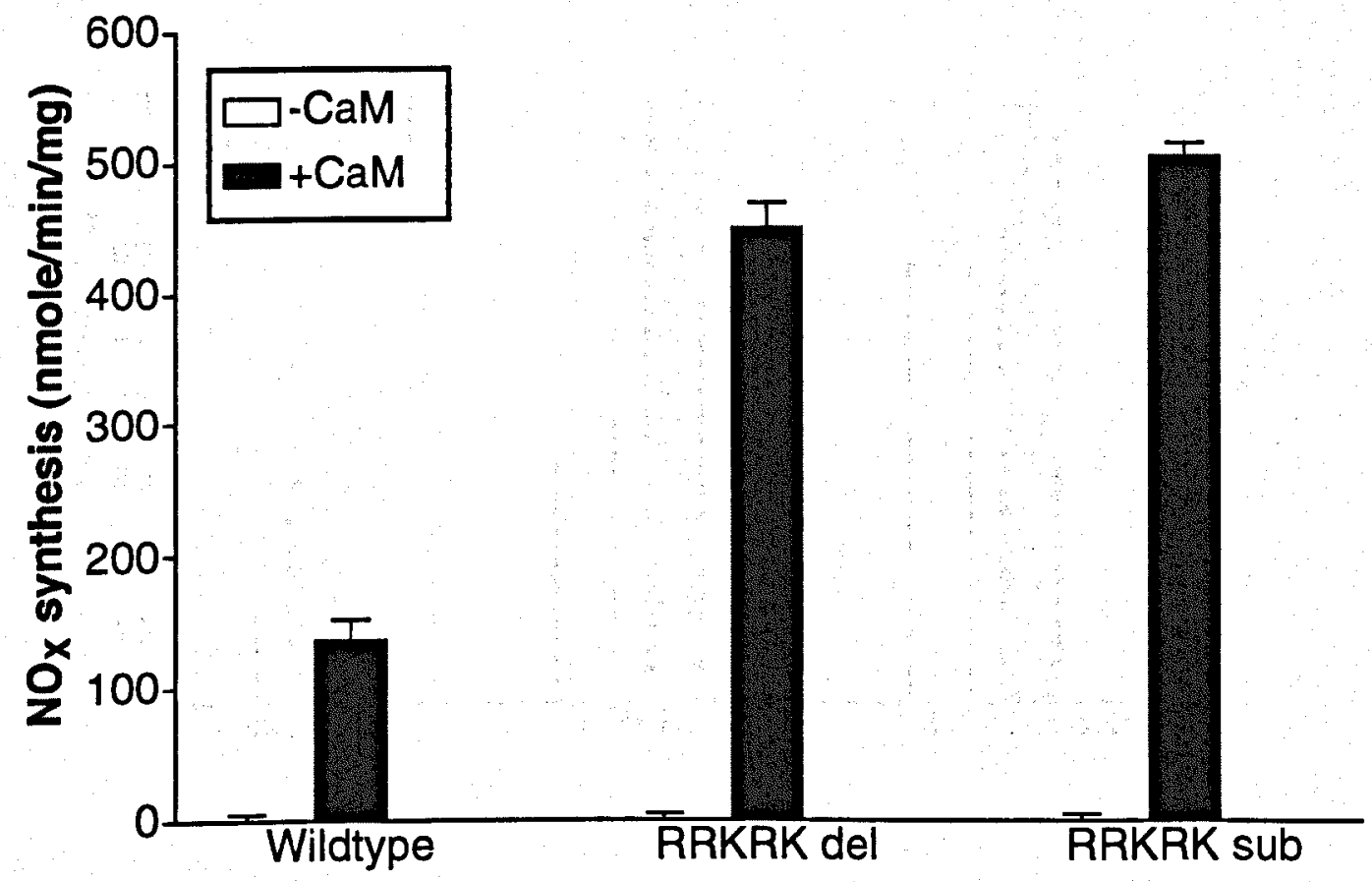

Fig. 3.23. Rates of NO synthesis of wildtype, RRKRK del and RRKRK sub eNOS. All experiments were carried out in triplicate using 10 pmoles of NOS, $250 \mathrm{nM} \mathrm{CaM}$ and optimal $\mathrm{Ca}^{2+}$ concentrations $(100 \mu \mathrm{M})$. CaM-free measurements of activity were obtained in the presence of $5 \mathrm{mM}$ EGTA and rates are derived from the mean of two separate preparations of each enzyme measured in triplicate 

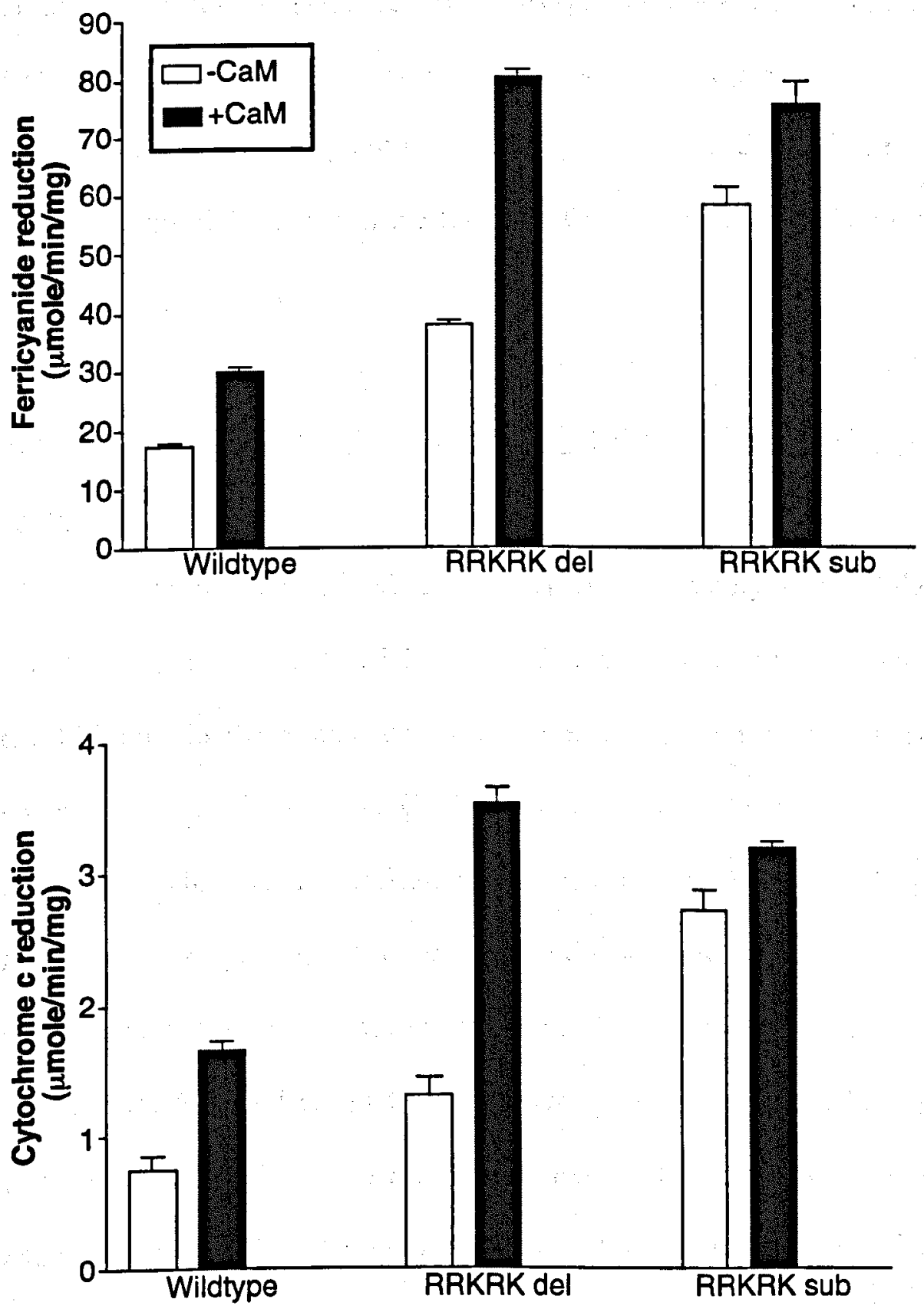

Fig. 3.24. Reductase activities of wildtype, RRKRK del and RRKRK sub eNOS. All experiments were carried out using $250 \mathrm{nM} \mathrm{CaM}$ and optimal calcium concentrations $(100 \mu \mathrm{M})$. Amounts of NOS added were 1 pmole and 0.1 pmole per well for cytochrome $c$ reduction (Panel B) and ferricyanide reduction (Panel A), respectively. CaM-free measurements of activity were obtained in the presence of 5 mM EGTA and rates are derived from the mean of two separate preparations of each enzyme assayed in triplicate. 
B) and eNOS activity (Fig. 3.25, panel A) were performed using an identical array of $\mathrm{Ca}^{2+}$ buffer solutions and assay conditions, permitting direct comparison of observed $\mathrm{Ca}^{2+}$-dependencies. Assays of CaM-binding to eNOS and RRKRK mutants revealed no significant differences in the levels of $\mathrm{Ca}^{2+}$ required to initiate formation of the $\mathrm{CaM} / \mathrm{eNOS}$ protein complex.

\subsubsection{Does it Affect the EC50 for $\mathrm{Ca}^{2+}$ if the CaM-eNOS Complexes are Pre-} formed?

Experiments described in the previous section only assessed the formation of eNOS complexes at defined levels of free $\mathrm{Ca}^{2+}$. Under physiological conditions, CaM-binding to eNOS, and hence activation, would occur during the rising phase of a spike in intracellular $\mathrm{Ca}^{2+}$. eNOS-CaM complexes would then dissociate, and hence inactivate eNOS as intracellular $\mathrm{Ca}^{2+}$ returned to basal levels. We therefore investigated any potential difference in the $\mathrm{Ca}^{2+}$-dependence curve for eNOS activity, if we looked at activation vs. inactivation. Activities of wildtype and RRKRK del eNOS were assessed uniquely under each of two conditions. Firstly, CaM-eNOS complexes were allowed to pre-form at maximal levels of $\mathrm{Ca}^{2+}(\approx 1 \mu \mathrm{M})$ prior to addition to the $\mathrm{Ca}^{2+}$-chelating buffers. Secondly, $\mathrm{eNOS}\left(\approx 1 \mu \mathrm{M} \mathrm{Ca}^{2+}\right)$ was added to $\mathrm{Ca}^{2+}$-buffered solutions already containing CaM. For both wildtype and RRKRK sub eNOS, the two distinct experimental paradigms did not demonstrate any significant alteration in the level of free $\mathrm{Ca}^{2+}$ required for half maximal activity (Fig. 3.26). 


\subsubsection{Does the RRKRK Motif Affect the Rate of Dissociation of CaM-eNOS}

\section{Complexes}

The rate of dissociation of CaM-eNOS complexes was assessed using a $\left[{ }^{125} \mathrm{I}\right]-$ CaM binding assay, as described in the methods section. The rate of dissociation of eNOS-CaM complexes was slightly accelerated in mutants lacking the RRKRK motif compared to wildtype eNOS (Fig. 3.27, panels A and B). This may indicate a role for this highly basic in the interaction between eNOS and the acidic lobes of compacted CaM. Removal of this region might lead to a higher rate of CaM dissociation

\subsubsection{The RRKRK Motif and eNOS-HSP90 Association}

HSP90 has been reported to form a complex with eNOS and this is associated with a 2 to 3-fold increase in the rate of NO synthesis (Garcia-Cardena et al., 1998). As removal of the RRKRK motif results in an enzyme with a comparable increase in activity, we investigated a possible role for this motif in the eNOS-HSP90 association. HSP90 caused a concentration-dependent increase in activity of both wildtype and RRKRK mutant eNOS proteins. In both cases, the maximum increase was approximately $130 \%$ of that demonstrated by eNOS in the absence of HSP90 (Fig. 3.28). This was much smaller than the published increase and may be due to our use of recombinant HSP90, as opposed to the HSP90 purified from rat brain was used previously (Garcia-Cardena et al., 1998). 

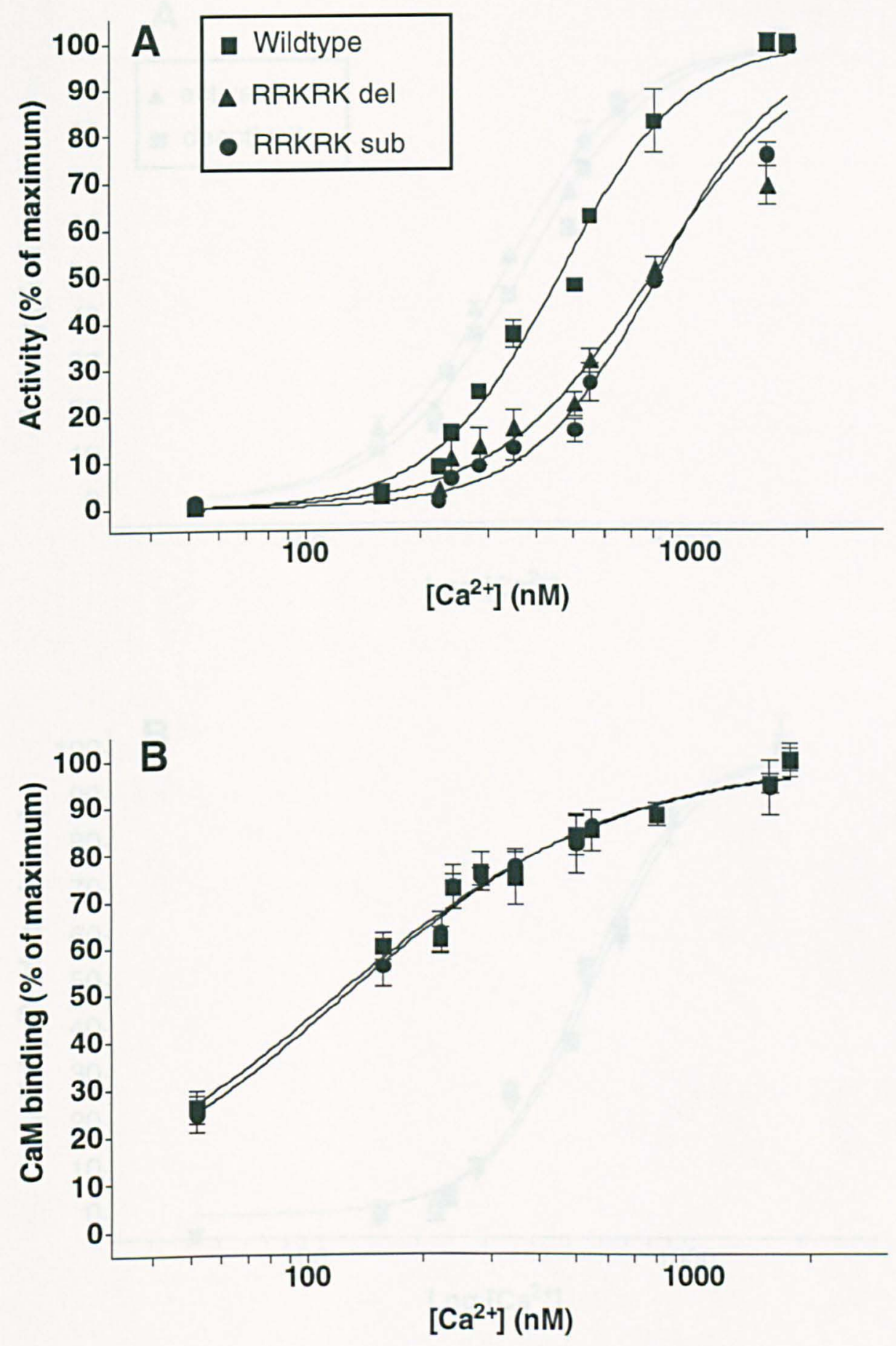

Fig. 3.25. Calcium-dependence of NO synthesis and calmodulin-binding for wild type and RRKRK mutant eNOSs. Panel A: NO synthesis is shown as a percentage of the maximum rate observed in the presence of $1.8 \mu \mathrm{M} \mathrm{Ca}^{2+}$ for each eNOS protein ( 8 pmol). Panel B: Calmodulin binding is depicted as a percentage of complete occupancy, where $100 \%$ represents a $1: 1$ ratio of bound CaM to eNOS ( 4 pmol). 

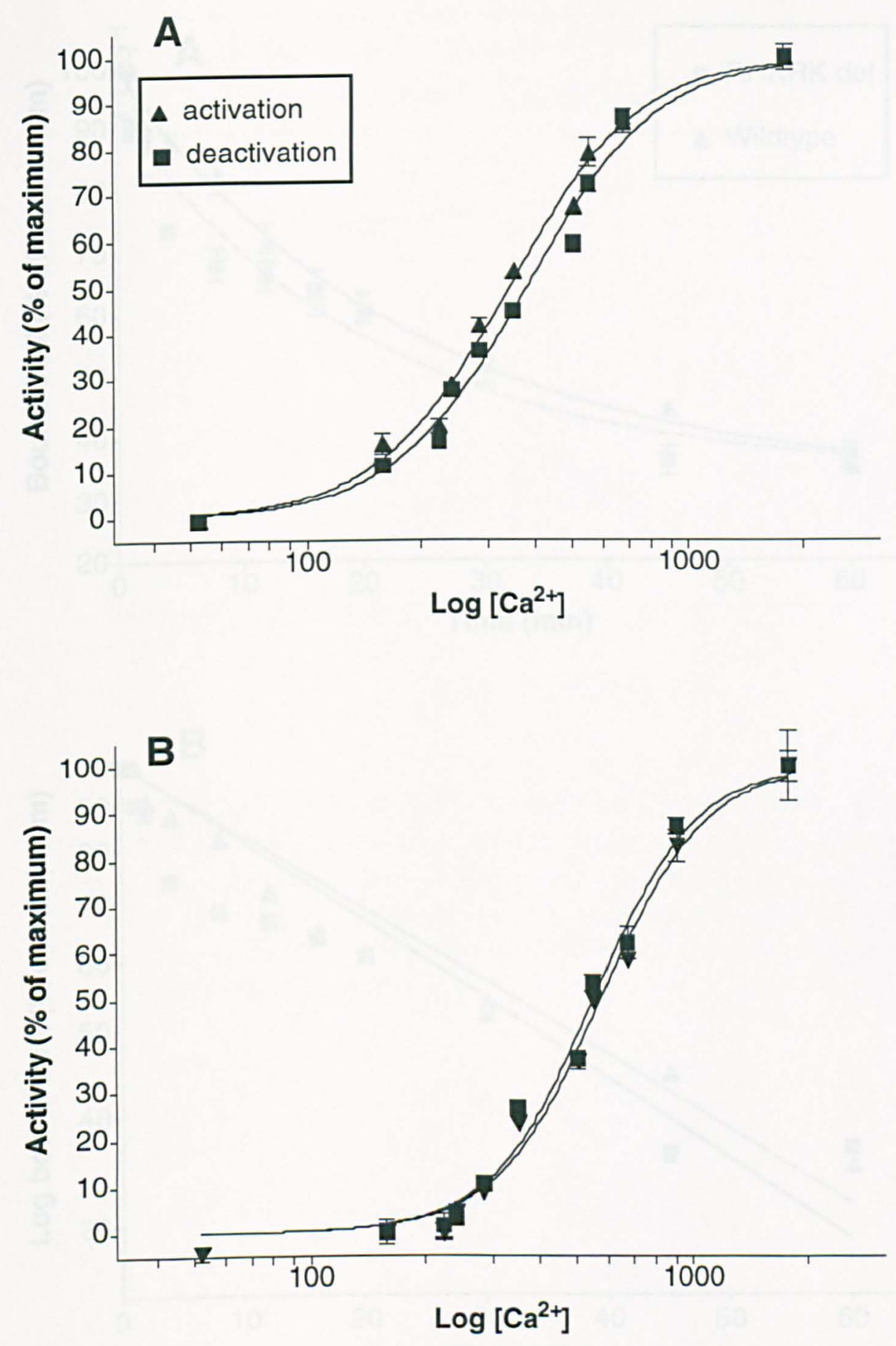

Fig. 3.26. $\mathrm{Ca}^{2+}$-dependence of activation vs. deactivation for wildtype and RRKRK del eNOS. $\mathrm{Ca}^{2+}$-dependence of eNOS activity was analyzed under conditions where CaM-eNOS complexes were allowed to form at defined levels of $\mathrm{Ca}^{2+}$ (activation) or to pre-form at $\approx 1 \mu \mathrm{M} \mathrm{Ca}^{2+}$ prior to transfer to the $\mathrm{Ca}^{2+}$ solutions (deactivation). Experiments were performed using $100 \mathrm{nM} \mathrm{CaM}$ and 8 pmoles of wildtype (Panel A) or RRKRK sub eNOS (Panel B).Values represent the mean of triplicate experiments \pm SEM. 

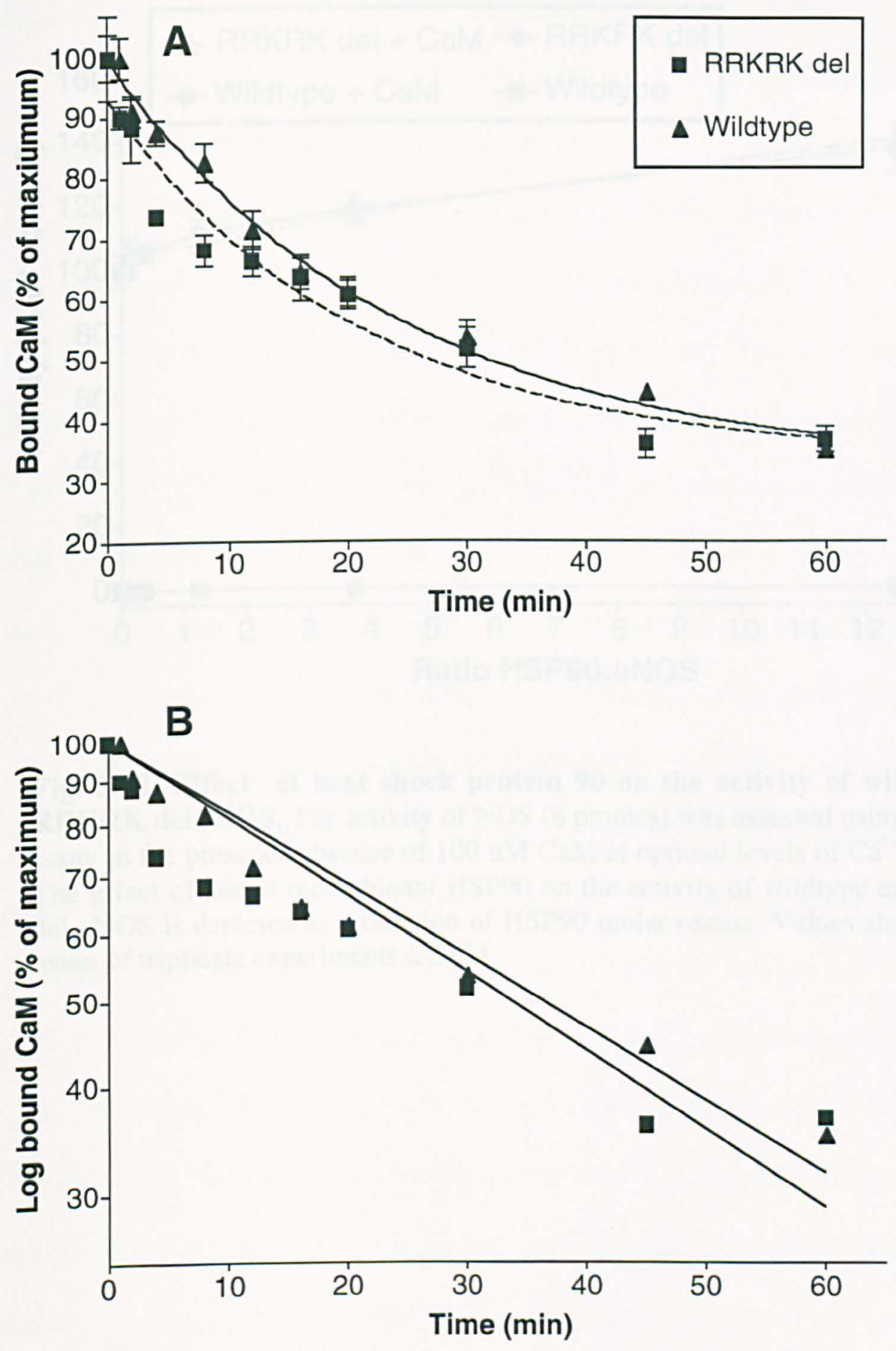

Fig. 3.27. Rate of $\left[{ }^{125} \mathrm{I}\right]-\mathrm{CaM}$ dissociation from wildtype and RRKRK del eNOS. Panel A: the rate of $\mathrm{CaM}$ dissociation from CaM-eNOS complexes at optimal levels of $\mathrm{Ca}^{2+}(100$ $\mu \mathrm{M}$ ) was assessed by following the time-dependent loss of bound $\left[{ }^{125} \mathrm{I}\right]-\mathrm{CaM}$ following the addition of a 3000 -fold molar excess of unlabeled CaM. CaM binding is expressed as a percentage of the maximum observed prior to the addition of unlabeled CaM. Panel B: A semi-log plot of the above data allows the derivation of a dissociation rate by linear regression. 


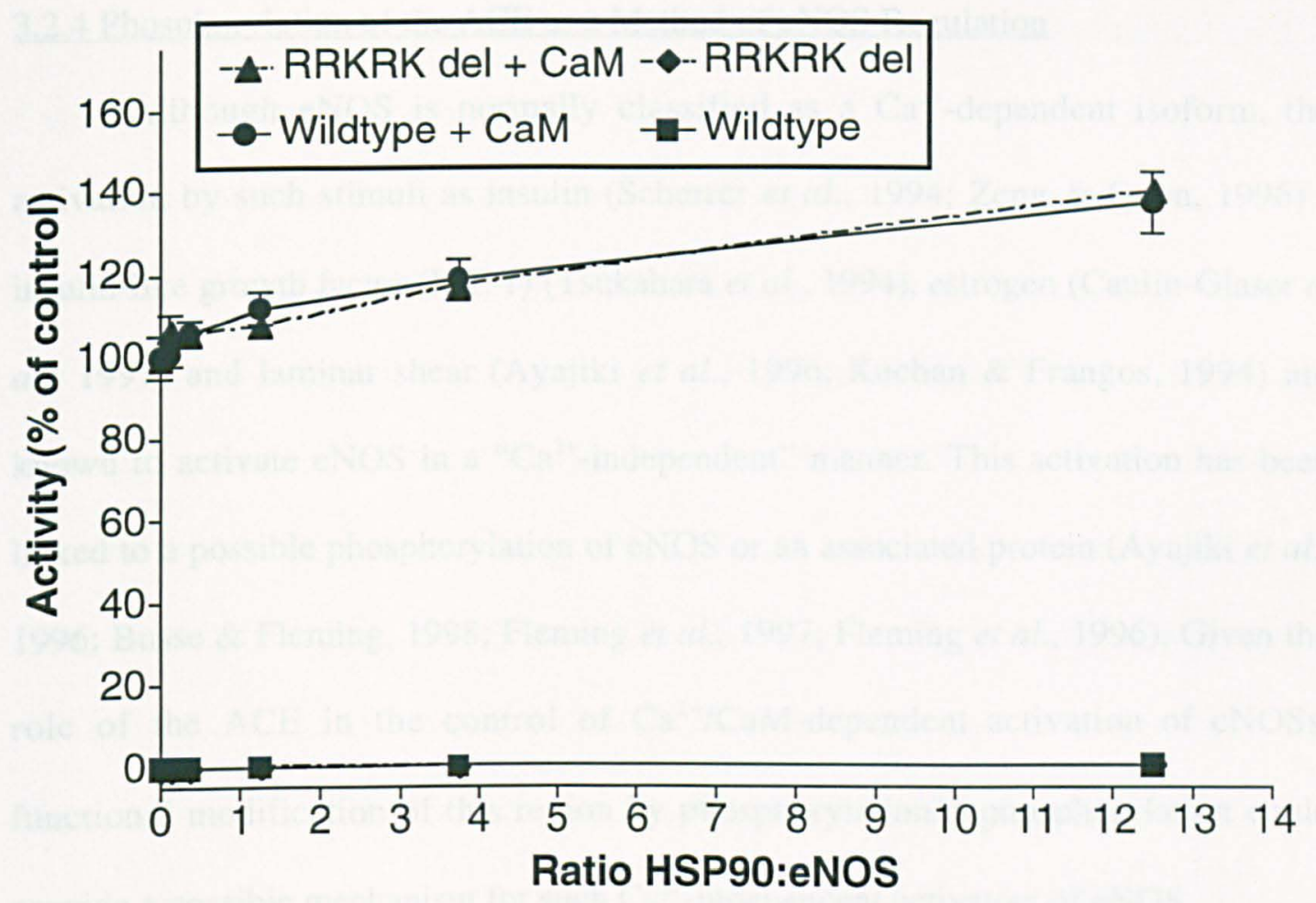

Fig. 3.28. Effect of heat shock protein 90 on the activity of wildtype and RRKRK del eNOS. The activity of NOS (8 pmoles) was assessed using the Griess assay in the presence/absence of $100 \mathrm{nM} \mathrm{CaM}$ at optimal levels of $\mathrm{Ca}^{2+}(100 \mu \mathrm{M})$. The effect of added recombinant HSP90 on the activity of wildtype and RRKRK del eNOS is depicted as a function of HSP90 molar excess. Values shown are the mean of triplicate experiments \pm SEM. 


\subsubsection{Phosphorylation of the ACE as a Method of eNOS Regulation}

Although eNOS is normally classified as a $\mathrm{Ca}^{2+}$-dependent isoform, the activation by such stimuli as insulin (Scherrer et al., 1994; Zeng \& Quon, 1996), insulin-like growth factor (IGF-1) (Tsukahara et al., 1994), estrogen (Caulin-Glaser $e t$ al., 1997) and laminar shear (Ayajiki et al., 1996; Kuchan \& Frangos, 1994) are known to activate eNOS in a "Ca ${ }^{2+}$-independent" manner. This activation has been linked to a possible phosphorylation of eNOS or an associated protein (Ayajiki et al., 1996; Busse \& Fleming, 1998; Fleming et al., 1997; Fleming et al., 1996). Given the role of the ACE in the control of $\mathrm{Ca}^{2+} / \mathrm{CaM}$-dependent activation of cNOSs, functional modification of this region by phosphorylation/dephosphorylation could provide a possible mechanism for such $\mathrm{Ca}^{2+}$-independent activators of eNOS.

\subsubsection{Putative phosphorylation sites in ACE Regions of cNOSs}

The ACE regions of cNOSs are rich in serine/threonine residues. Comparison of the full length sequences of eNOS and nNOS with the Prosite database (Swisprot, Expasy) identifies consensus phosphorylation sites for kinases including protein kinase A (PKA), CaM-dependent kinase II (CaM KII), casein kinase II (CK II), protein kinase C (PKC) and protein kinase B (PKB/Akt). Fig. 3.29 (panel A) shows the existence of a PKA site within the ACE of cNOSs, which is conserved across species. Another putative PKA phosphorylation site is present within the CaM binding domain of cNOSs (eNOS, Threonine ${ }_{494}$ ) (Fig. 3.29, panel B). Phosphorylation of a ser/thr residue within or near a CaM-binding site or CaM-binding regulation site (ACE) is a common mechanism of regulation for many CaM-dependent enzyme systems. Nonetheless, the functionality of the two PKA sites remains to be established. 
3.2.4.2 Attenuation of the Inhibitory Potency of $\mathrm{eNOS}_{612-632}$ by Phosphorylation at

\section{Consensus PKA sites}

To examine the possible functional significance of these two putative phosphorylation sites within the ACE of eNOS, synthetic peptides corresponding to eNOS $_{617-639}$ were synthesized in their native form, or phosphorylated at serine ${ }_{6.35}$ or serine $_{636}$. The ability of these peptides to inhibit $\left[{ }^{125} \mathrm{I}\right]-\mathrm{CaM}$ binding to nNOS was assessed as described previously. As expected, the native peptide potently inhibited CaM-binding to nNOS ( $\mathrm{IC}_{50} 5.56 \pm 1.03 \mu \mathrm{M}$ ) (Fig. 3.30), however, the phosphopeptides exhibited a substantially reduced ability to inhibit CaM-binding $\left(\mathrm{IC}_{50}>200\right.$ $\mu \mathrm{M})$. As these peptides mimic the inhibitory effect of the intrinsic ACE, and phosphorylation of the peptide at $\operatorname{Ser}_{635 / 636}$ attenuates this inhibitory effect, it is conceivable that phosphorylation of the intrinsic ACE may cause a similar loss in inhibitory function. We therefore investigated the significance of PKA-mediated phosphorylation on eNOS activity in vitro.

\subsubsection{In Vitro Phosphorylation of eNOS by PKA}

Although consensus sequences for PKA-mediated phosphorylation can be found within the amino acid sequence of eNOS, this does not necessarily mean that these sites are available targets of PKA. Hence, the ability of PKA to catalyze the in vitro phosphorylation of eNOS was assessed using ${ }^{32} \mathrm{P}$-labeled ATP. PKA-dependent phosphorylation of eNOS was demonstrated by a time-dependent incorporation of radiolabeled-phosphate into the $130 \mathrm{kDa}$ band on SDS-PAGE gels (Fig. 3.31, panel A). Liquid scintillation counting of the excised bands allowed the quantification of phosphate incorporation into eNOS and revealed a final stoichiometry of $\approx 1$ phosphate per eNOS monomer (Fig. 3.31, panel B). The stoichiometry and the rate of 
phosphorylation were unaffected by whether the substrate eNOS was CaM-bound or CaM-free. Control experiments demonstrated that omission of PKA from the reaction prevented ${ }^{32} \mathrm{P}$-incorporation into eNOS.

Although the validity of eNOS as a substrate of PKA was now established, the location of the phosphorylated sites identified in the above study were unknown. To establish whether any of the PKA sites involved were $\operatorname{Ser}_{635}, \operatorname{Ser}_{636}$ or $\mathrm{Thr}_{494}$, we produced mutant eNOS proteins in which one or more of these residues was negated by mutation to an alanine. The incorporation of phosphate was markedly diminished by mutation of $\operatorname{Ser}_{635 / 636}($ Fig 3.32). Additionally, when all three residues were mutated to alanines no PKA-mediated phosphate incorporation could be detected (Fig. 3.33) To confirm the identity of $\operatorname{Ser}_{635}$ as one of the PKA phosphorylation sites within eNOS, we used the masses of trypsinolysis fragments predicted by in silica digestion and utilized selected ion recording electrospray ionization mass spectrometry (SIRESI-MS) to analyze the fragments from actual digests. Analysis of tryptic fragments of phosphorylated eNOS showed the presence of a peak of the correct mass predicted by phosphorylation at $\operatorname{Ser}_{635}$ or $\operatorname{Ser}_{636}$ (Fig. 3.34). This peak was not present in the digest of unphosphorylated eNOS. However, due to the use of SIR-MS, the presence of other undetected phosphorylated peptides cannot discounted.

\subsubsection{Effect of in vitro Phosphorylation on eNOS Activity}

Now it was established that PKA could mediate the phosphorylation of eNOS at a site within the $\mathrm{ACE}$, we performed assays to ascertain whether phosphorylation altered eNOS-function. 
We hypothesized a model based upon the regulation of CaM-dependent kinase II (Brickey et al., 1994), in which a sequestered phosphorylation site on the ACE is exposed upon $\mathrm{Ca}^{2+} / \mathrm{CaM}$ binding and that this ACE-phosphorylation would thus provide a mechanism to sustain eNOS activity after $\mathrm{Ca}^{2+}$ levels have returned to the basal. We therefore looked at the effect of PKA-mediated phosphorylation on the observed $\mathrm{Ca}^{2+}$-dependence of eNOS. This was done under the conditions described in Part III, whereby pre-formed CaM-eNOS complexes were placed into specific EGTA/Ca $\mathrm{Ca}^{2+}$-buffered solutions that provide a range of $\left[\right.$ free $\left.\mathrm{Ca}^{2+}\right]$, from subnanomolar to $1.8 \mu \mathrm{M}$.

Thus experiments were performed to examine the effect of PKA-mediated phosphorylation on the level of free $\mathrm{Ca}^{2+}$ required for activation of eNOS, previously treated with ATP/PKA or ATP/inactivated PKA. The extent of phosphorylation in each case was assessed using an identical reaction containing ${ }^{32} \mathrm{P}$-labeled ATP (see inset). However, the levels of free $\mathrm{Ca}^{2+}$ required for half-maximal activation of eNOS were found to be indistinguishable, irrespective of whether PKA-dependent phosphorylation of eNOS occurred (Fig. 3.35).

We also examined the effect of phosphorylation on the maximal catalytic rates exhibited by eNOS. Previously treated under the phosphorylating or mockphosphorylating conditions described above, eNOS was assayed for its relative rate of NO synthesis. None of these treatments were found to cause a significant alteration in specific activity of eNOS (Fig. 3.36), although rates of NO synthesis were modestly diminished in all cases compared to untreated eNOS. Despite no apparent effect on NO synthesis, it is possible that phosphorylation by PKA could mediate an effect 
exclusively within the reductase domain. Hence, the effect of these PKA treatments on reductase function was assessed by eNOS-mediated cytochrome c (Fig. 3.37, panel A) and ferricyanide reduction (panel B). No significant effect upon reductase function was observed with any of the phosphorylating or mock-phosphorylating treatments.

\subsubsection{Mutation of $\operatorname{Ser}_{635}$ to Mimic Phosphorylation}

Although the mutation/mass spectrometry studies identified $\operatorname{Ser}_{635 / 636}$ as a site of in vitro PKA-mediated phosphorylation, it was also evident that at least one additional residue $\left(\mathrm{Thr}_{494}\right)$ also underwent phosphorylation. The relative stoichiometry of phosphorylation of these two residues in wildtype eNOS was also unclear. Additionally, the presence of $\mathrm{Mg}^{2+}$ ions in the kinase reaction mixture complicates the interpretation of $\mathrm{Ca}^{2+}$-dependence curves, due to the ability of $\mathrm{Mg}^{2+}$ to occupy $\mathrm{Ca}^{2+}$ binding sites of CaM. To circumvent these complications, we took advantage of an established method to mimic the specific phosphorylation of $\operatorname{Ser}_{635}$. Glutamate has a similar size and charge pattern to a phosphoserine and accordingly, substitution of a Glu for a Ser residue often mimics the effect of phosphorylation. We therefore created two mutant eNOS proteins, one expressing the phosphoserine-mimic $\left(\mathrm{Glu}_{635}\right)$ and a conservative control mutation $\left(\mathrm{Ala}_{635}\right)$. The effect of these mutations on eNOS activity were then assessed.

Expression of these proteins was problematic, yields of $\mathrm{Glu}_{635}$ and $\mathrm{Ala}_{635}$ eNOS were much lower than those expected (100 $\mu \mathrm{g} /$ liter of culture). This was not rectified by the addition of mild detergents, such as CHAPS. Despite the modest yields, we attempted to characterize $\mathrm{Glu}_{635}$ and $\mathrm{Ala}_{635}$ eNOS. 
Specific activity measurements demonstrated the very low level of activity of Glu $_{635}$ and $A \mathrm{Al}_{635}$ mutants ( $25 \pm 5$ and $19 \pm 2 \mathrm{nmole} / \mathrm{min} / \mathrm{mg}$ protein), compared to wildtype eNOS (135 $\pm 17 \mathrm{nmole} / \mathrm{min} / \mathrm{mg}$ protein) (Fig. 3.38). The low yield and specific activity of this protein made the assessment of the $\mathrm{Ca}^{2+}$-dependence curve for activation by $\mathrm{CaM}$ unfeasible. However, $\mathrm{Glu}_{635}$ eNOS did exhibit a reduced rate of $\left.{ }^{125} \mathrm{I}\right]$-CaM dissociation, compared to the wildtype eNOS (Fig. 3.39). 
620 C S D P L V S S W R R K

621 C S D P L V T S W R K K

617 C S D P L V S S W R R K K"

617 C S D P L V S S WR R K.

620 C S D P L V S S W R K K

619 C S D P L V S S W R R K that S N T D S A G A L G T Bovine eNOS

1 -

PKA/PKG Consensus

\section{B}

485 S A S K G A G V Th" K F K E V A N A V K I S A L dog eNOS

486 S G T K G T G I ThE K F F E V A N A V K I S A S L Guinea pig eNoS

482 S A A K G T G I T⿰亻丨 K K K E V A N A V K I S A S L Human eNOS

482 S A A K A A I T K K K F E A N A V K I S A S L mouse eNOS

485 S A A K G T I A K K F K E V A N A V K I S A S L pig eNOS?

484 S A T K G A I T T K K F K V A N A V K I S A L L Bovine eNOS

1 -

PKA/PKG Consensus

Fig. 3.29. Conservation of PKA/PKG consensus sites in eNOS. Alignments of regions of the FMN-insertion (Panel A) and the CaM-binding site (Panel B) of eNOS showing conservation of the key residues making up the PKA/PKG consensus. 


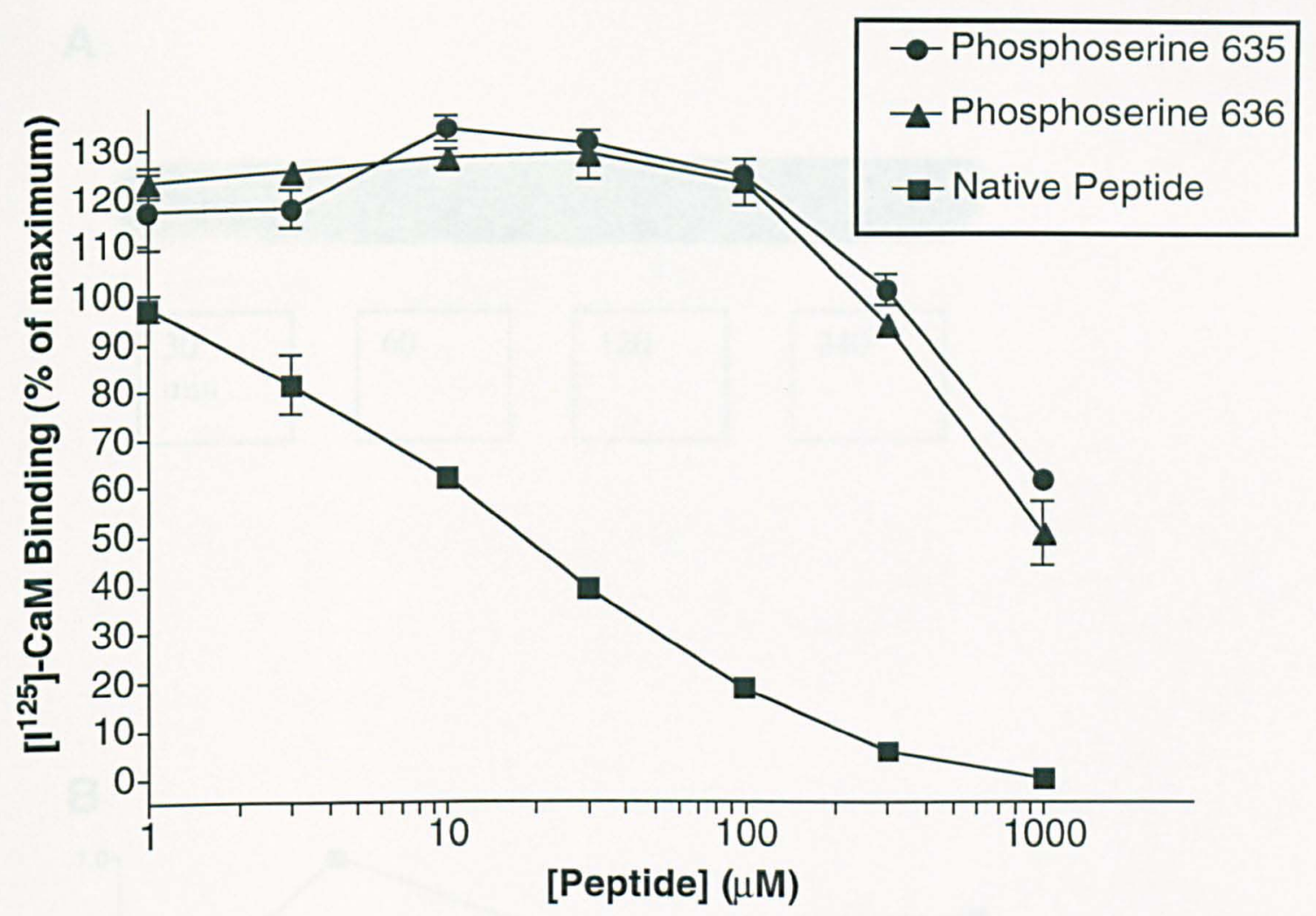

Fig. 3.30. Attenuation of the inhibitory potency of eNOS $\mathrm{S}_{617-639}$ by phosphorylation at consensus cAMP-dependent protein kinase sites. Binding of $\left[{ }^{125} \mathrm{I}\right]-\mathrm{CaM}$ to 1 pmole of nNOS was assessed as a function of the concentration of the indicated peptide. CaM binding is expressed as a percentage of the maximum binding in the absence of added peptide. Values are corrected for non-specific $\mathrm{CaM}$ binding and are the mean of triplicate samples \pm SEM. 

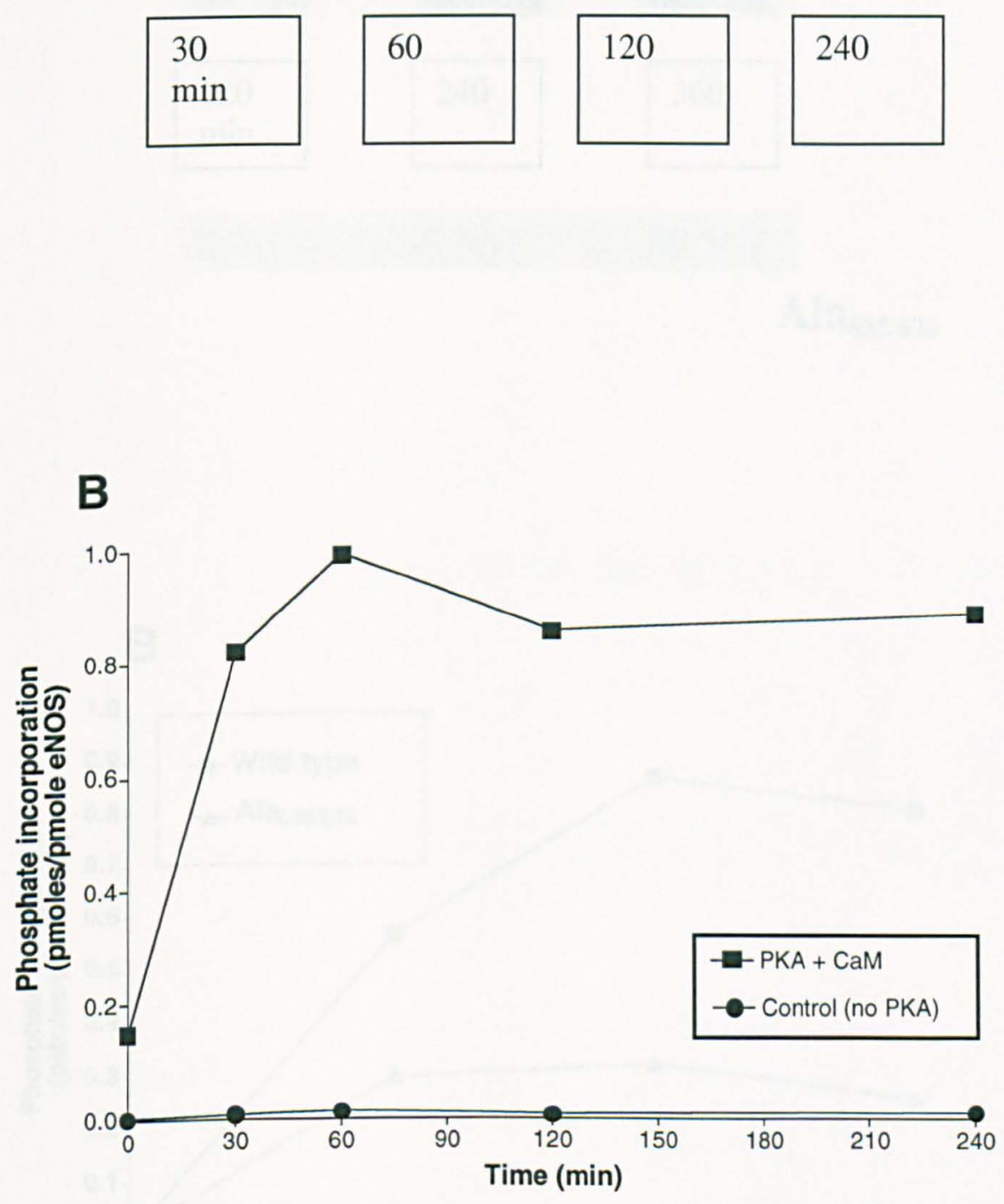

Fig. 3.31 ${ }^{32} \mathrm{P}$-Phosphate incorporation into wildtype eNOS by PKA. Phosphate incorporation studies were performed as described in Methods. Panel A: Autoradiographs of the dried gels showing phosphate incorporation into eNOS in a time-dependent manner. Panel B: The same gel bands were then excised and phosphate incorporation quantified by liquid scintillation counting. 


\section{Wild type}

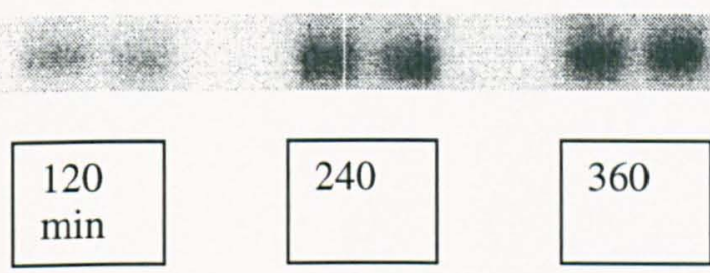

$\mathrm{Ala}_{635 / 636}$

B

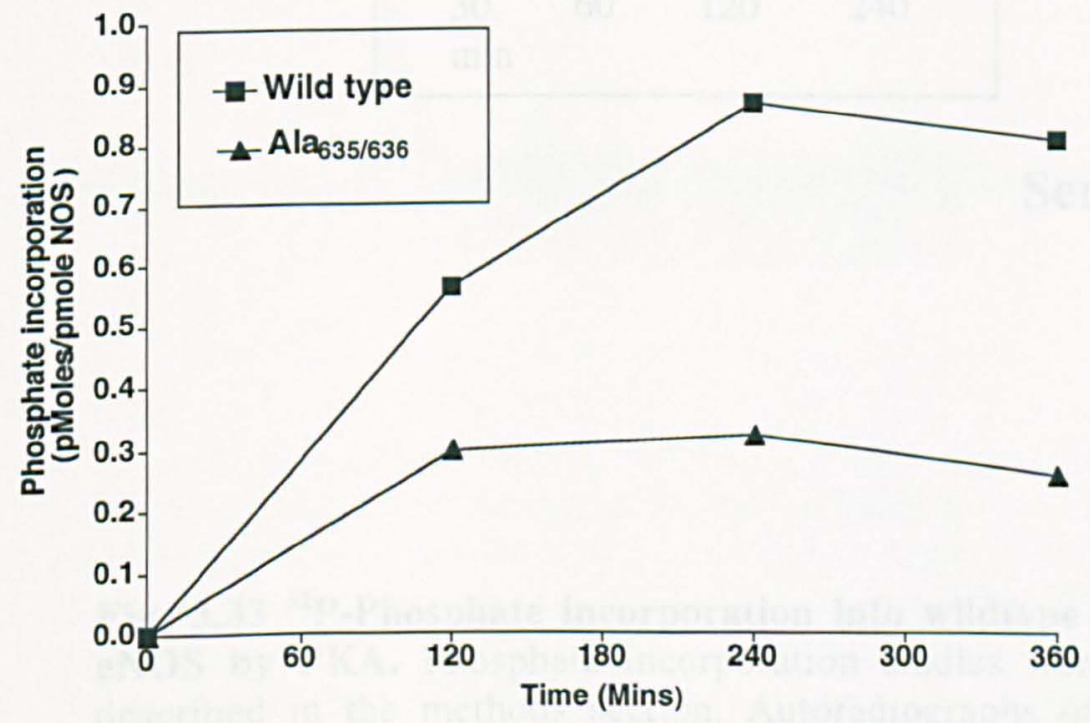

Fig. 3.32 ${ }^{32} \mathrm{P}$-Phosphate incorporation into wildtype and $\mathrm{Ala}_{635 / 636}$ eNOS by PKA. Phosphate incorporation studies were performed as described in Methods. Panel A: Autoradiographs of the dried gels showing phosphate incorporation into eNOS in a time-dependent manner. Panel B: The same gel bands were then excised and phosphate incorporation quantified by liquid scintillation counting. 


\begin{tabular}{|c|c|c|c|}
\hline $\begin{array}{l}30 \\
\min \end{array}$ & 60 & 120 & 240 \\
\hline
\end{tabular}

$\operatorname{Ser}_{494 / 635 / 636}$

Fig. $3.33{ }^{32} \mathrm{P}$-Phosphate incorporation into wildtype and $\mathrm{Ala}_{494 / 635 / 636}$ eNOS by PKA. Phosphate incorporation studies were performed as described in the methods section. Autoradiographs of the dried gels demostrate phosphate incorporation into wildtype eNOS in a timedependent manner. Mutation of Serine ${ }_{494 / 633 / 636}$ to alanines blocks phosphate incorporporation. 


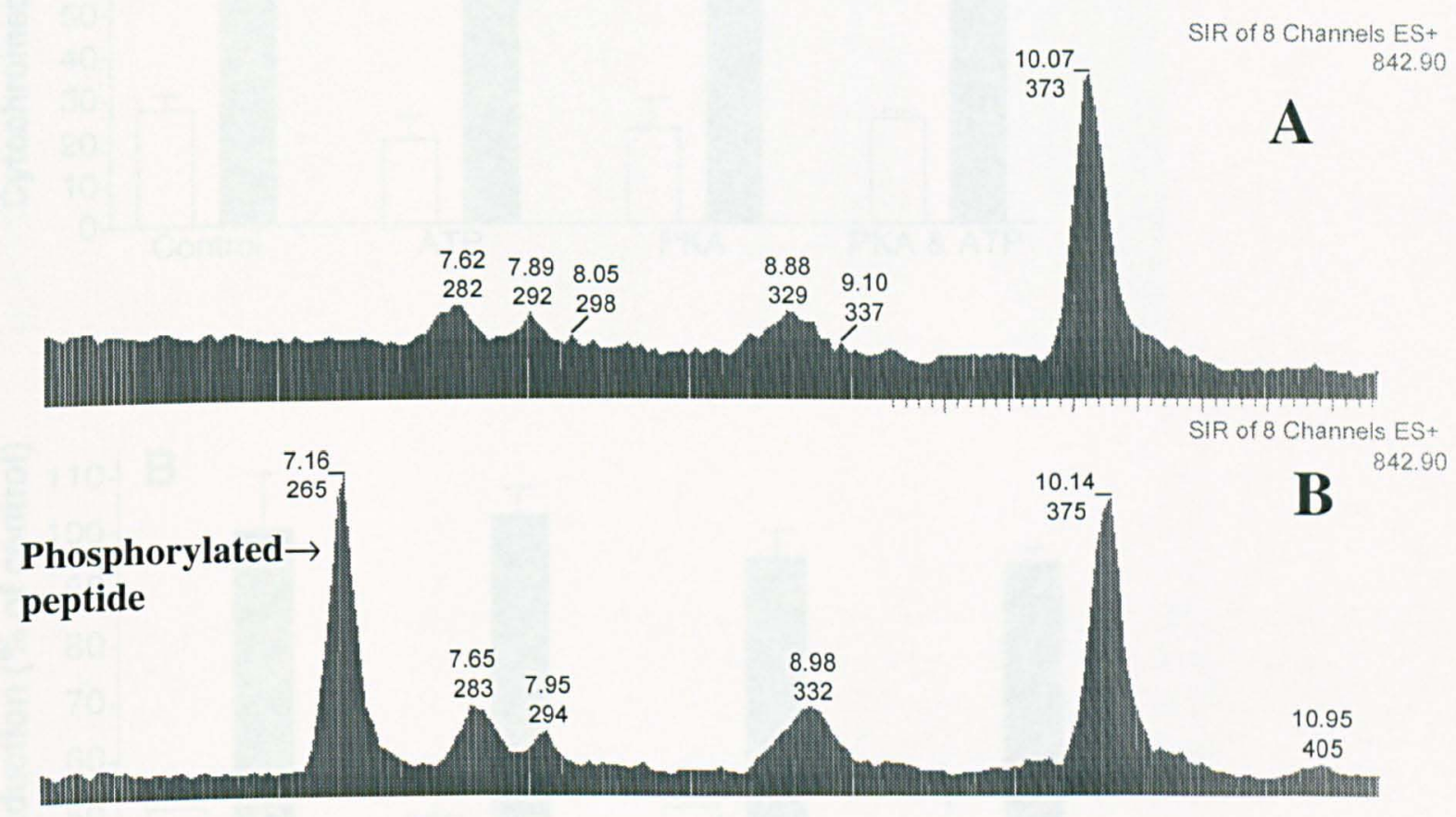

Fig. 3.34. Confirmation of Serine ${ }_{635 / 636}$ as a site of in vitro PKA-mediated phosphorylation by electrospray ionization mass spectrometry using selected ion recording. Selected ion recording mass spectrometry (SIR-MS) analysis of trypsin digests of native (Panel A) and PKA/ATP-exposed eNOS (Panel B), demonstrating the appearance of a peak at $842.9 \mathrm{Da}(\mathrm{m} / \mathrm{z}=2)$, corresponding to phosphorylated eNOS $_{633-647}$ (KESSNTDAGALGTLR). A peak with a similar retention time is not present in the unphosphorylated digest. For SIR-MS analysis, both native and phosphorylated proteins contained PKA and eNOS (150 pmoles); ATP was present only in the latter case. Samples were eluted from a reverse phase $\left(\mathrm{C}_{18}\right)$ HPLC column feeding directly into the mass spectrometer. 

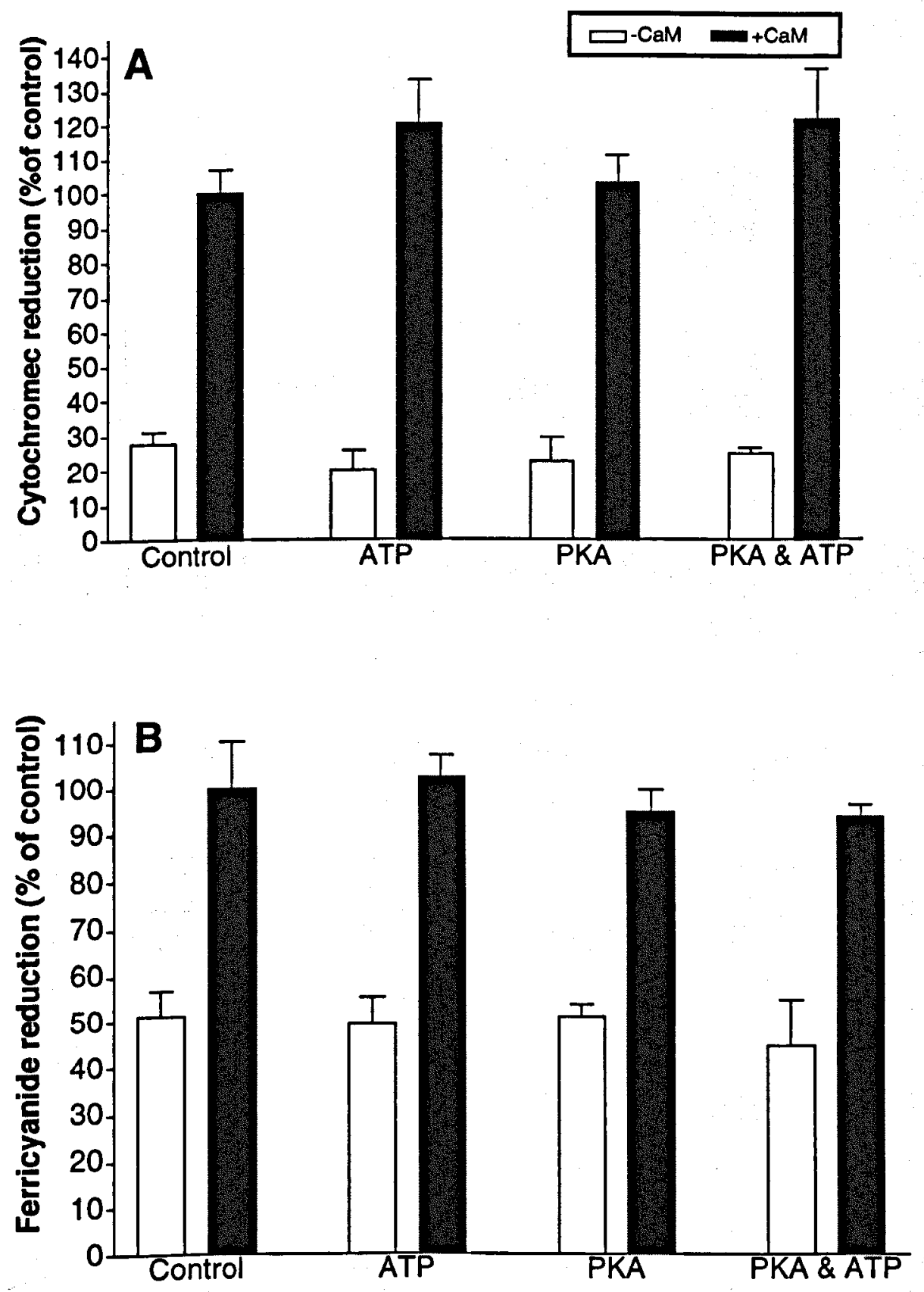

Fig. 3.37. Effect of PKA-mediated phosphorylation on the rates of ferricyanide and cytochrome $c$ reduction. eNOS was treated as described in Fig. 3.36 and assessed for cytochrome c (Panel A) and ferricyanide (Panel B) reductase activities. Reactions were performed using $250 \mathrm{nM}$ $\mathrm{CaM}, 100 \mu \mathrm{M} \mathrm{Ca}^{2+}$ and 1 pmole (cytochrome c) or 0.1 pmole (ferricyanide) of eNOS. Activity in the absence of CaM was assessed in the presence of 5 mM EGTA. Values are the mean of triplicate experiments \pm SEM. 


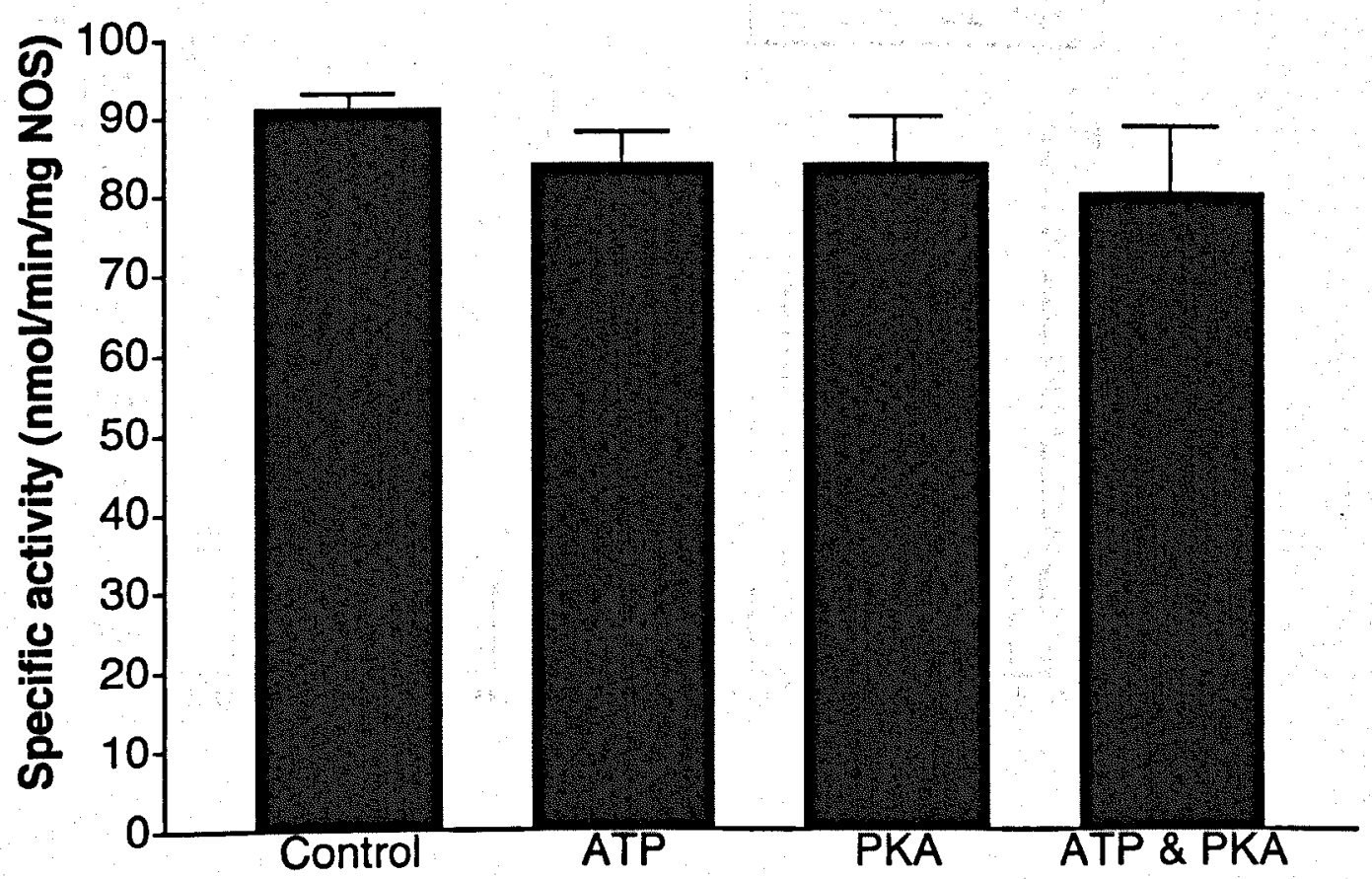

Fig. 3.36. Effect of PKA-mediated phosphorylation on the rate of NO synthesis by eNOS. eNOS ( 8 pmoles) was treated for 20 min with either phosphorylation buffer only (control), ATP/heat inactivated PKA (ATP), PKA only or ATP/PKA and then incubated in NOS assay buffer containing $250 \mathrm{nM} \mathrm{CaM}$ and optimal $\mathrm{Ca}^{2+}$ concentrations $(100 \mu \mathrm{M})$. Values shown are the mean of triplicate experiments \pm SEM. 

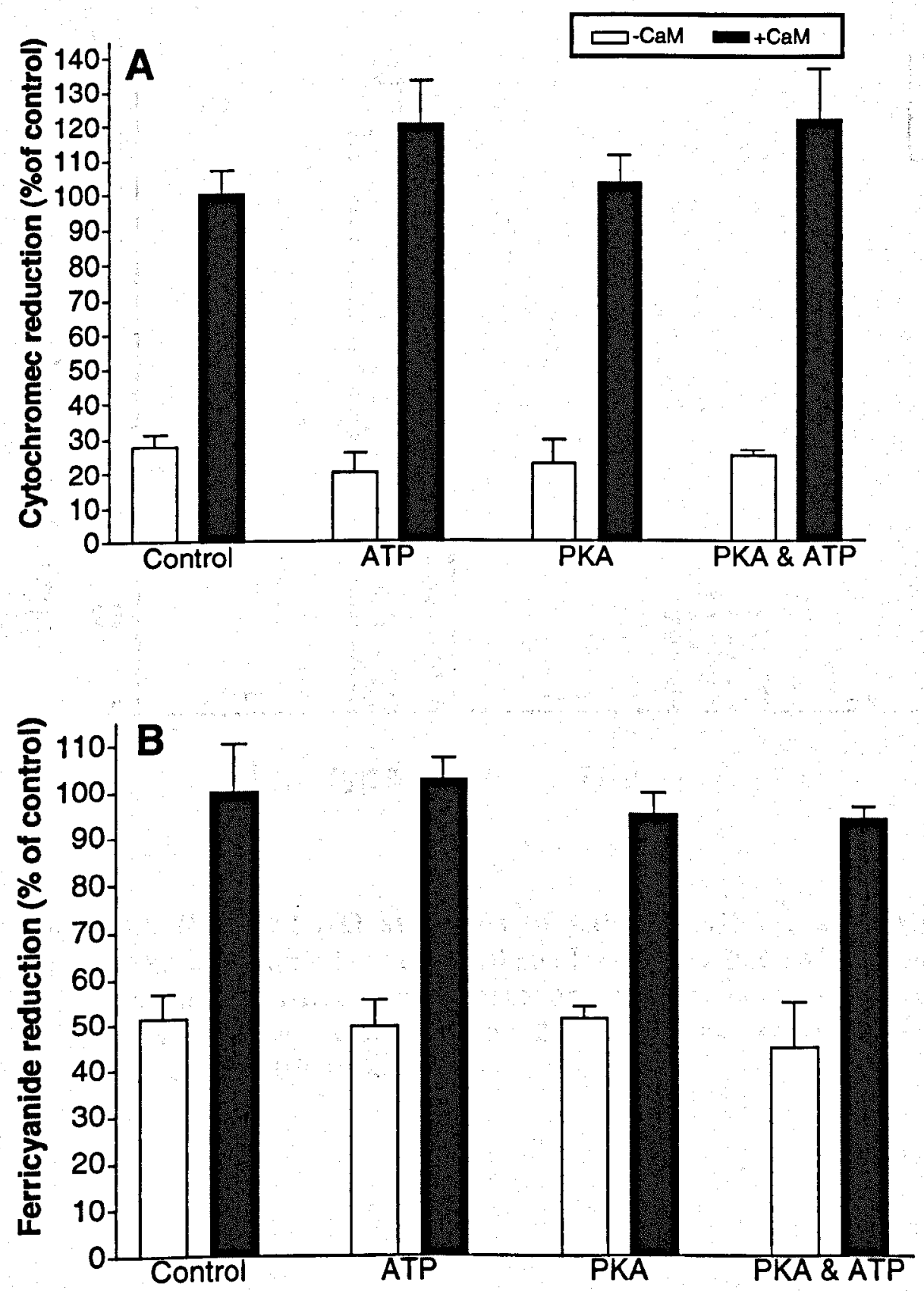

Fig. 3.37. Effect of PKA-mediated phosphorylation on the rates of ferricyanide and cytochrome $c$ reduction. eNOS was treated as described in Fig. 3.36 and assessed for cytochrome $\mathrm{c}$ (Panel A) and ferricyanide (Panel B) reductase activities. Reactions were performed using $250 \mathrm{nM}$ $\mathrm{CaM}, 100 \mu \mathrm{M} \mathrm{Ca}^{2+}$ and 1 pmole (cytochrome c) or 0.1 pmole (ferricyanide) of eNOS. Activity in the absence of $\mathrm{CaM}$ was assessed in the presence of 5 mM EGTA. Values are the mean of triplicate experiments \pm SEM. 


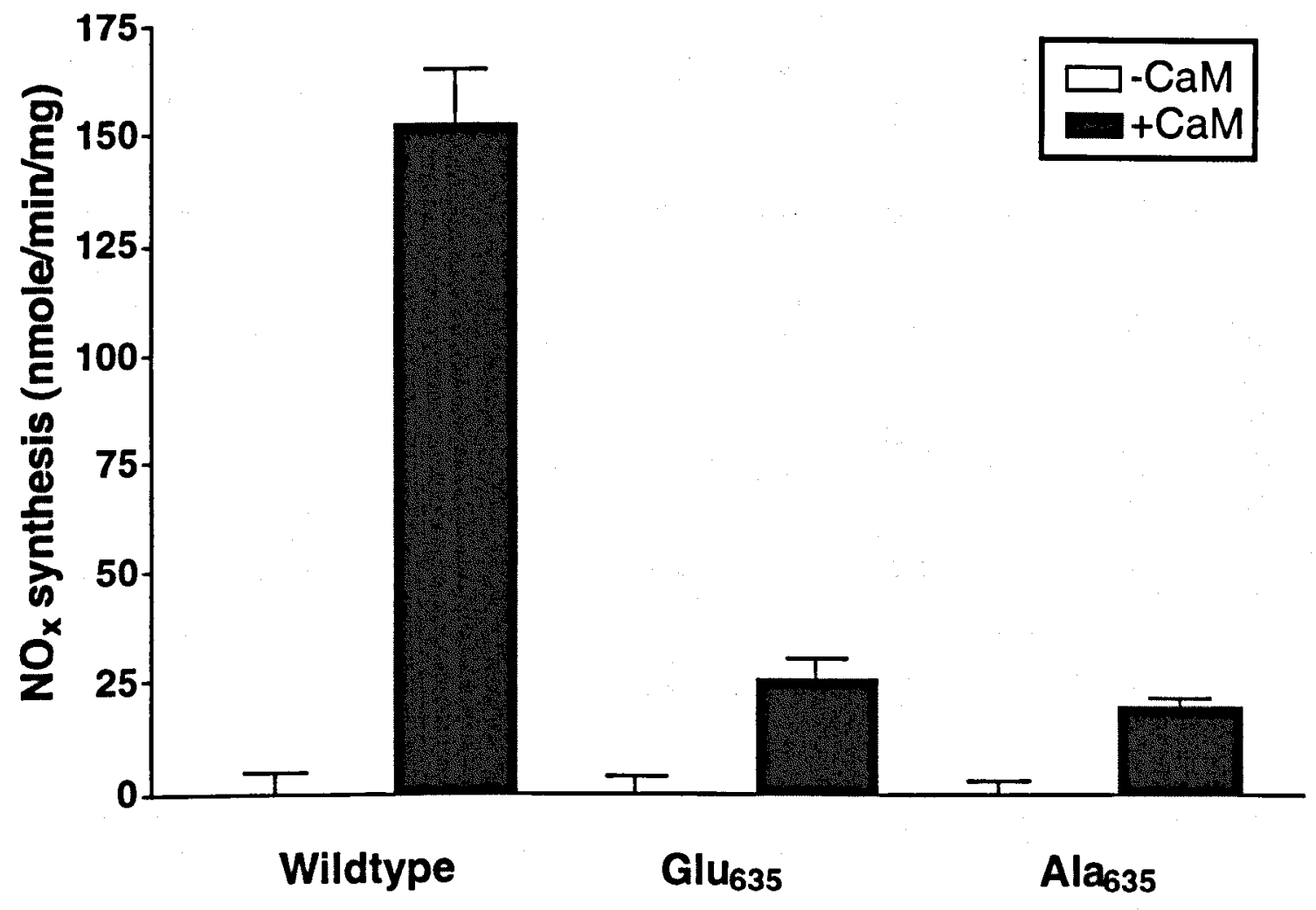

Fig. 3.38. Rates of NO synthesis of wildtype $\mathrm{Glu}_{635}$ and $\mathrm{Ala}_{635}$ eNOS. All experiments were carried out using 10 pmoles of NOS, $250 \mathrm{nM} \mathrm{CaM}$ and maximal $\mathrm{Ca}^{2+}$ concentrations. CaM-free measurements of activity were obtained in the presence of 5 mM EGTA and rates are derived from the mean of two separate preparations of each enzyme, assayed in triplicate. 

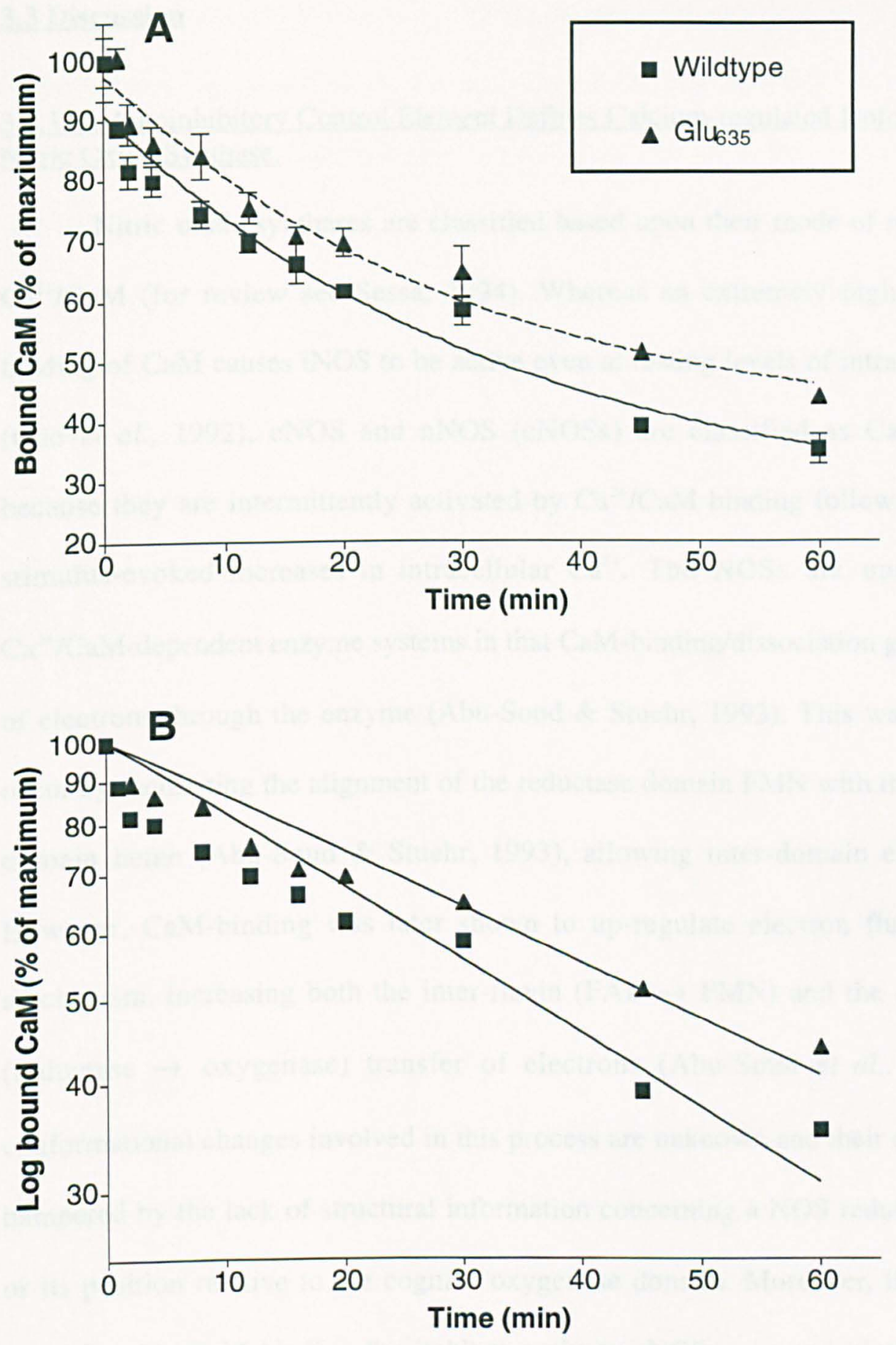

Fig. 3.39. Rate of $\left[{ }^{125} \mathrm{I}\right]-\mathrm{CaM}$ dissociation from wildtype and $\mathrm{Glu}_{635}$ eNOS. Panel A: the rate of CaM dissociation from CaM-eNOS complexes formed at maximal levels of $\mathrm{Ca}^{2+}$ $(100 \mu \mathrm{M})$ was assessed by following the time-dependent loss of bound $\left[{ }^{125} \mathrm{I}\right]-\mathrm{CaM}$ following the addition of a 3000 -fold molar excess of unlabeled CaM. CaM binding is expressed as a percentage of the maximum observed prior to the addition of unlabeled CaM. Panel B: A semi-log plot of the above data allows the derivation of a dissociation rate by linear regression. 


\subsection{Discussion}

3.3.1An Autoinhibitory Control Element Defines Calcium-regulated Isoforms of Nitric Oxide Synthase.

Nitric oxide synthases are classified based upon their mode of regulation by $\mathrm{Ca}^{2+} / \mathrm{CaM}$ (for review see Sessa, 1994). Whereas an extremely high affinity for binding of CaM causes iNOS to be active even at resting levels of intracellular $\mathrm{Ca}^{2+}$ (Cho et al., 1992), eNOS and nNOS (cNOSs) are classified as $\mathrm{Ca}^{2+}$-dependent because they are intermittently activated by $\mathrm{Ca}^{2+} / \mathrm{CaM}$ binding following transient stimulus-evoked increases in intracellular $\mathrm{Ca}^{2+}$. The NOSs are unique among $\mathrm{Ca}^{2+} / \mathrm{CaM}$-dependent enzyme systems in that CaM-binding/dissociation gates the flow of electrons through the enzyme (Abu-Soud \& Stuehr, 1993). This was thought to occur by facilitating the alignment of the reductase domain FMN with the oxygenase domain heme (Abu-Soud \& Stuehr, 1993), allowing inter-domain electron flux. However, CaM-binding was later shown to up-regulate electron flux by a dual mechanism, increasing both the inter-flavin (FAD $\rightarrow$ FMN) and the inter-domain (reductase $\rightarrow$ oxygenase) transfer of electrons (Abu-Soud et al., 1994). The conformational changes involved in this process are unknown and their elucidation is hampered by the lack of structural information concerning a NOS reductase domain or its position relative to the cognate oxygenase domain. Moreover, the molecular basis for this CaM-binding "switch" to activate cNOSs was completely unknown prior to this study. In contrast, the activation of most other $\mathrm{Ca}^{2+} / \mathrm{CaM}$-dependent enzymes has been shown to rely upon the displacement of an intrinsic autoinhibitory control element (Jarrett \& Madhavan, 1991). 
Herein we have shown that cNOSs possess a polypeptide insertion within their FMN-binding domains that is: 1) unique to NOS isoforms that are regulated by transient $\mathrm{Ca}^{2+} / \mathrm{CaM}$ binding; 2) positioned adjacent to the $\mathrm{CaM}$-binding domain, as assessed by molecular modeling; 3) an impediment to $\mathrm{Ca}^{2+} / \mathrm{CaM}$ binding and hence, enzyme activation; 4) displaced upon the binding of $\mathrm{Ca}^{2+} / \mathrm{CaM}$. We propose that the tonic inhibition of cNOSs is reliant upon the occupation of key regulatory sites on cNOS by the autoinhibitory insertion. CaM binding causes the displacement of the insertion from these sites, thus activating NOS catalysis by "disinhibition". Close proximity of the inhibitory insertion would provide for an exceedingly high local concentration, forcing the equilibrium to favor the insertion-bound/inhibited state in the absence of bound $\mathrm{Ca}^{2+}-\mathrm{CaM}$. This provides a possible explanation for the detection of basal activity in purified eNOS or nNOS in the absence of bound $\mathrm{Ca}^{2+} / \mathrm{CaM}$ (Moncada et al., 1989; Nathan \& Xie, 1994), as it is conceivable that a low steady-state of the disinhibited conformation of cNOS may exist in the absence of bound- $\mathrm{Ca}^{2+} / \mathrm{CaM}$.

Following up on our identification of a putative ACE in the FMN-binding domain of cNOSs, recent reports have confirmed the autoinhibitory function (Chen \& Wu, 2000; Daff et al., 1999; Montgomery et al., 2000; Nishida \& Ortiz de Montellano, 1999). Nishida and coworkers (Nishida \& Ortiz de Montellano, 1999) engineered a series of $\mathrm{cNOS}$ constructs in which the ACE was deleted. For several other $\mathrm{Ca}^{2+} / \mathrm{CaM}$-dependent enzymes, removal of an autoinhibitory domain eliminated tonic inhibition, resulting in an increased level of enzymatic activity in the absence of bound CaM. Removal of the ACE from cNOSs, either by deletion of the ACE or by substitution with the reductase domain of iNOS, however, did not result in $\mathrm{Ca}^{2+} / \mathrm{CaM}$ - 
independent NO synthesis (Daff et al., 1999; Montgomery et al., 2000; Nishida \& Ortiz de Montellano, 1999). Nonetheless, maximal $\mathrm{Ca}^{2+} / \mathrm{CaM}$-independent reductase domain activity was found to increase 2-3-fold, compared to the corresponding wild type enzyme. The $\mathrm{Ca}^{2+} / \mathrm{CaM}$-independent activities of mutant cNOSs were at least equal to the $\mathrm{Ca}^{2+} / \mathrm{CaM}$-bound activities exhibited by their respective wild type proteins. Additionally, the eNOS ACE deletion mutants (eNOS $\Delta$ ) were found to have a reduced $\mathrm{Ca}^{2+}$-dependence for activity $\left(\mathrm{EC}_{50}=20 \mathrm{nM}\right)$ compared to wild type eNOSs $\left(E C_{50}=150 \mathrm{nM}\right)($ Nishida \& Ortiz de Montellano, 1999). Substitution of either the entire reductase domain from eNOS or nNOS, with the homologous sequence of iNOS (devoid of an inherent insertion peptide), resulted in a similarly reduced $\mathrm{Ca}^{2+}$ dependence $\left(\mathrm{EC}_{50}=10 \mathrm{nM}\right.$ in each case). Essentially identical effects on $\mathrm{Ca}^{2+}$ dependence were obtained for nNOS constructs expressing a deletion of the ACE (Daff et al., 1999; Montgomery et al., 2000).

cNOS proteins without the peptide insertion exhibited enhanced $\mathrm{Ca}^{2+} / \mathrm{CaM}$ independent reductase activities and synthesized NO at reduced levels of $\mathrm{Ca}^{2+}$, compared to wild type proteins. These characteristics define the peptide insert as an autoinhibitory control element that contributes to the observed physiological $\mathrm{Ca}^{2+}-$ dependence of cNOSs. However, as cNOS constructs lacking the autoinhibitory peptide still require $\mathrm{CaM}$ for NO synthesis, it was also apparent that removal/displacement of the ACE alone is insufficient to elicit enzyme activation. Hence, CaM-induced conformational changes in addition to ACE displacement are necessary for activation of NO production. These additional actions of CaM may include alignment of FMN and heme regions between companion subunits in cNOS dimers, as well as alignment of FAD and FMN domains within subunits; alone or 
together, these CaM-induced modifications would promote catalytically productive electron flux into cNOS oxygenase domains (Abu-Soud \& Stuehr, 1993).

The proposed control mechanism specifies that the binding of $\mathrm{Ca}^{2+} / \mathrm{CaM}$ would elicit the displacement of the ACE: this would predict that the presence of the ACE reduces the affinity for $\mathrm{Ca}^{2+} / \mathrm{CaM}$ binding to cNOSs. Reciprocally, the absence of the ACE in the sequence of iNOS would obviate the otherwise expected steric hindrance to $\mathrm{Ca}^{2+} / \mathrm{CaM}$-binding and contribute to the observed high affinity $\mathrm{Ca}^{2+} / \mathrm{CaM}$ binding exhibited by iNOS at all levels of intracellular $\mathrm{Ca}^{2+}$. Studies of polypeptides corresponding to the CaM-binding sites of eNOS and iNOS, and of chimeras, in which the CaM-binding sequence of one NOS isoform is substituted for the corresponding region of another (Ruan et al., 1996; Venema et al., 1996), indicate that the $\mathrm{Ca}^{2+}$-dependence and affinity of $\mathrm{CaM}$ binding is dictated by elements in addition to the recognized CaM-binding sequence. These results have been interpreted to imply the existence of auxiliary CaM-binding regions on iNOS, accounting for the high-affinity CaM-binding exhibited by iNOS. However, the information provided herein recommends an alternative interpretation of these results, that the absence of the ACE from the sequence of iNOS contributes to an enhanced CaM affinity at low levels of free $\mathrm{Ca}^{2+}$.

The studies of ACE-deleted cNOSs outlined above provide some insight into the underlying mechanism of the $\mathrm{Ca}^{2+}$-independent activity exhibited by iNOS. Chimeric cNOSs expressing the reductase domain of iNOS (including the CaMbinding site), like iNOS itself, exhibited activity even in the presence of 5 mM EGTA (Nishida \& Ortiz de Montellano, 1998). If the absence of the ACE is the only reason 
for the $\mathrm{Ca}^{2+}$-independence of iNOS, then cNOS chimera lacking the ACE would also be expected to exhibit $\mathrm{Ca}^{2+}$-independent activity. However, such chimera, although requiring a reduced level of free $\mathrm{Ca}^{2+}$ for maximal activity than the respective wild type cNOSs, were nonetheless inactive in the presence of a calcium-chelator, EGTA. Therefore the $\mathrm{Ca}^{2+}$-independent activity of iNOS cannot be explained simply by the absence of an ACE, but may arise from two scenarios. Either, iNOS possesses regions in addition to the canonical CaM-binding site that contribute to the interaction with bound $\mathrm{CaM}$, or, as yet unidentified regions of cNOSs contribute to the proposed steric hindrance of $\mathrm{Ca}^{2+} / \mathrm{CaM}$ binding and hence, $\mathrm{cNOS}$ activation.

In support of the former view, it is notable that known proteins which exhibit high-affinity CaM-binding at low levels of free $\mathrm{Ca}^{2+}$ often have two regions that contribute to the CaM-binding site. If thiswere the case for iNOS, a suitable candidate for this auxiliary site must lie immediately adjacent to the acknowledged CaMbinding site. Coded for by a single exon, analogous to the two exons of cNOSs coding for the ACE, a small (17 amino acids in human iNOS), highly acidic (6 Arg/Lys) region exists. Close proximity to the conventional CaM-binding site and a charge pattern that is complimentary to the exposed acidic region of activated CaM, make this a good candidate for additional interactions with bound CaM. The smaller size of this region, compared to the ACE, would preclude the spatial hindrance of CaMbinding that we attribute to the ACE. Deletion or mutation of this region in iNOS or replacement with the ACE of a cNOS, would provide a valuable test of wether this peptide provides a second CaM binding site in iNOS. Interestingly, an alternative alignment of the FMN-binding regions of NOSs (Chen \& Wu, 2000) identifies a region in iNOS that is analogous to the region NOS $_{607-613}$ (KSYKIRF) within the 
ACE. A modified mutation of the entire ACE of eNOS, in which only the regions of the ACE on either side of this motif are deleted ( $\mathrm{eNOS}_{594-606}$ and $\left.\mathrm{eNOS}_{614645}\right)$ has been constructed and investigated for function (Chen $\& \mathrm{Wu}, 2000$ ). This mutant exhibits high-affinity CaM binding and maintains $60 \%$ of its maximal level of NO synthesis in the presence of $5 \mathrm{mM}$ EGTA. This region of iNOS, and regions immediately flanking it, are rich in basic residues and therefore could serve as an accessory CaM-binding site. This mutant was co-expressed with CaM and, like wild type iNOS was found to bind CaM as an intrinsic co-factor. Reciprocally, the absence of this region from the previously described ACE-deletions (Daff et al., 1999; Montgomery et al., 2000; Nishida \& Ortiz de Montellano, 1999) may explain the lack of predicted $\mathrm{Ca}^{2+}$ independent activity.

Our results, considered in the context of subsequent mutational analysis as noted above, may also provide an understanding of the intricacies of the $\mathrm{Ca}^{2+}$ independent activity exhibited by iNOS in vivo. Although it is known that incorporation of CaM into iNOS is a co-translational event, it remains unknown why CaM binding to iNOS appears to be $\mathrm{Ca}^{2+}$-independent. One possibility is that iNOS binds to, and is activated by, $\mathrm{Ca}^{2+}$-free $\mathrm{CaM}$, nonetheless, this is unlikely given the observed decrease in iNOS activity in the presence of high concentrations of EGTA. More likely is that $\mathrm{Ca}^{2+}$-saturated $\mathrm{CaM}$ is incorporated into iNOS, and once bound exhibits a much higher affinity for $\mathrm{Ca}^{2+}$ binding, resulting in residual activity even in the presence of EGTA. Hence, the term " $\mathrm{Ca}^{2+}$-independent" may be a misleading term when it comes to iNOS and other high-affinity CaM-binding proteins, such as phosphorylase kinase (Cohen et al., 1978; Grand et al., 1981) and myosin light chain kinase (Krueger et al., 1998). 
Given the proposed manner of ACE-mediated inhibition, whereby the ACE functions as an impediment to $\mathrm{Ca}^{2+} / \mathrm{CaM}$-binding, it is surprising that some $\mathrm{ACE}$ derived peptides can inhibit iNOS activity, albeit at much higher concentrations than required to inhibit cNOS activity. As mentioned previously, iNOS binds CaM with a remarkably high affinity, boiling in SDS-containing, calcium-chelating buffers is required to uncouple CaM-iNOS complexes (Cho et al., 1992). Inhibition of NOS activity by ACE-derived peptides is therefore, extremely unlikely to involve the displacement of bound CaM from iNOS. As a direct interaction can be demonstrated between CaM and ACE-derived peptides at high concentrations, one possibility is that the peptides interact with iNOS-bound CaM in situ, in the process disrupting iNOS activity. There is also the possibility that the reductase domain of iNOS, although devoid of an ACE, still contains vestigial binding sites required for ACE-mediated inhibition. This may provide clues to the evolutionary derivation of the NOS family of proteins. It is conceivable that iNOS either evolved from the cNOSs via a deletion event or, reciprocally, that the cNOSs were derived from iNOS by an insertional event. The presence of vestigial ACE-binding sites in iNOS would favor the former scenario. It is notable that phylogenetic trees, created by computer-assisted analysis of NOS isoform divergence, indicates that ACE-containing NOS (cNOSs) is ancestral to ACE-free NOS (iNOS).

Although the putative site(s) of $\mathrm{ACE} / \mathrm{cNOS}$ inhibitory interaction are unknown, several inferences as to the general locale and characteristics of such regions can be made. Firstly, $\mathrm{Ca}^{2+} / \mathrm{CaM}$ - dependent regulation remains intact in isolated nNOS reductase domain, making it likely that some sites of ACE-interaction 
reside within this domain (Newton et al., 1998). Secondly, given the highly basic nature of the ACE (especially of eNOS), sites of interaction are likely to carry an excess of negative charge. Thirdly, by analogy to CaM-dependent kinase II, in which the autoinhibitory domain binds with the substrate binding site, the ATP binding site and bound-CaM (Brickey et al., 1994), the presence of multiple sites of cNOS/ACE interaction may be envisioned.

Considering the probability that ACE-binding sites would be acidic in nature, one obvious candidate would be the surface of bound $\mathrm{CaM} ; \mathrm{Ca}^{2+}$-bound $\mathrm{CaM}$ has an excess of exposed negative charges and also satisfies the criteria of proximity to the ACE. Examination of the amino acid sequence of cNOSs shows the presence of acidic "patches" located within the oxygenase domain. Other possible sites of interaction are the regions of the FMN-binding domain that flank the ACE or are directly adjacent to the CaM-binding domain. The location of some ACE-binding sites in the oxygenase domain is consistent with two observations. Firstly, chimeric NOSs expressing the reductase domain of cNOSs paired with the iNOS oxygenase domain exhibit a much-reduced $\mathrm{Ca}^{2+}$-dependence. In such chimera, the substituted iNOS oxygenase domain may be devoid of ACE interaction sites, nullifying the inhibitory effect that would otherwise be seen with wild type cNOSs. Secondly, the pattern of trypsinolysis observed for CaM-free isolated nNOS reductase domain, indicates that the ACE is already "free" (liu and Gross, unpublished observation), conceivably by removal of sites of ACE interaction within the oxygenase domain. Future mutational analysis of these putative binding sites in cNOSs, coupled with increased structural studies will be required to definitively identify sites of $\mathrm{ACE} / \mathrm{cNOS}$ interaction. 
Our initial studies, combined with subsequent mutational approaches, confirm the role of the FMN-insertion as an autoinhibitory control element (ACE). The absence of this region results in an increased basal-, and $\mathrm{Ca}^{2+} / \mathrm{CaM}$-stimulated electron flux through the reductase domain of both eNOS and nNOS. Secondly, the ACE of the cNOSs appears to be largely, but not solely, responsible for the $\mathrm{Ca}^{2+}$ dependence of activity exhibited by these enzymes. Important questions that remain are the autoinhibitory mechanism of the ACE and the location and identity of the key ACE-peptide interaction sites.

Our study of ACE-derived peptides suggests that binding may involve at least two conserved regions within the ACE. One is the basic motif RRKRK of eNOS (truncated to RK in nNOS) and the other is located at the well conserved N-terminal aspect of the ACE (EERKSYKVRF). ACE-derived peptides that express the Nterminal region weakly inhibit cNOS activity, while those expressing the RRKRK motif are considerably more potent. The possible roles of these regions are considered below.

3.3.2 Deletion of a Pentabasic Sequence Within the Autoinhibitory Control Element of eNOS Causes Enhanced Activity of the Reductase Domain and an Increased Rate of NO Synthesis.

From the deletion studies discussed in the previous section, we can now attribute two functions to the ACE of cNOSs: (1) govern the rate of electron flux through the 
reductase domain and (2) increasing the observed $\mathrm{Ca}^{2+}$ concentration required for activation by $\mathrm{Ca}^{2+} / \mathrm{CaM}$. Whether identical or distinct, structural motifs of the ACE mediating (1) and (2) was considered.

As noted above, alignment of the ACE regions of cNOSs clearly shows two distinct regions of conservation. An N-terminal segment, conserved throughout the cNOSs and a pentabasic motif (RRKRK) unique to eNOSs, (although a truncated dibasic form can be found in nNOSs, RK). To investigate the function of the RRKRK motif we expressed and characterized mutant eNOS proteins in which this motif was deleted or substituted with five Ala residues. In comparison to wild type eNOS, mutants lacking this motif were $2-3$ fold more active in terms of NO synthesis, stemming directly from an increased rate of electron flux through the reductase domain ( as assessed from a concomitant increase in cytochrome $\mathrm{c}$ and ferricyanide reduction). Both basal (CaM-independent) and CaM-activated reductase activity was increased by the mutation, although bound-CaM was still required for the synthesis of NO. It is notable that calcium $\mathrm{EC}_{50}$ values for activation by $\mathrm{CaM}$ were only slightly increased in the mutants and $\mathrm{Ca}^{2+}$-dependence for $\mathrm{CaM}$-binding were completely unaffected by the mutation. Additionally, eNOS proteins lacking the RRKRK motif exhibited a slightly increased rate of CaM dissociation.

The characteristics of mutant eNOSs that lack the RRKRK motif, combined with analyses of NOS mutants by other labs, helps to resolve some of the issues concerning $\mathrm{cNOS}$ regulation by $\mathrm{Ca}^{2+} / \mathrm{CaM}$ and the ACE. Deletion of the entire ACE from eNOS provided an interesting but unanticipated result, in that the resulting CaMbound protein exhibited a greatly increased maximal rate of NO synthesis, compared 
to CaM-bound wild type eNOS (Nishida \& Ortiz de Montellano, 1999). When the corresponding mutation was made in nNOS, the maximal catalytic rate of CaM-bound enzyme was unaffected by the absence of the ACE (Daff et al., 1999; Montgomery et al., 2000). This was unexpected, given our hypothesis that CaM-binding would completely relieve the inhibitory effect of the autoinhibitory domain and therefore the CaM-bound mutant and wild type eNOS were predicted to exhibit similar catalytic rates. However, it was previously shown that the NOS isoforms have fundamentally different rates of maximal enzymic activity. Specifically, nNOS (Bredt \& Snyder, 1990; Gerber \& Ortiz de Montellano, 1995; Harteneck et al., 1994; Roman et al., 1995) and iNOS (Fossetta et al., 1996; Gerber et al., 1997; Hevel et al., 1991; Stuehr et al., 1991) have been shown to exhibit 5-10 fold higher overall activity than eNOS (Chen et al., 1997; List et al., 1997; Martasek et al., 1996; Pollock et al., 1991; Rodriguez-Crespo et al., 1996; Rodriguez-Crespo \& Ortiz de Montellano, 1996) (The specific activities we observed for wild type NOSs agreed well with published reports). The reduced rate of NO synthesis by eNOS was shown to arise specifically from a reduced maximal activity of the reductase domain (Nishida \& Ortiz de Montellano, 1998), although the structural basis for this impaired reductase activity was unknown. We now appreciate that the autoinhibitory peptide is responsible for the inherently slower rate of reductase domain electron flux in eNOS.

NOS protein chimera containing the eNOS reductase domain, but with a deletion of the ACE, displayed an increase in maximal reductase domain activity (assessed by cytochrome $\mathrm{C}$ and ferricyanide reduction) compared to wild type eNOS. It is notable that for eNOS $\Delta$, the CaM-free reductase activity actually exceeded that observed with CaM-bound wildtype eNOS (Nishida \& Ortiz de Montellano, 1999). 
This implies that the inhibitory effects of the ACE are never fully removed by CaM binding to wildtype eNOS, explaining the relatively low level of reductase domain activity and hence NO synthesis by eNOS. Accordingly, the structural features which impair reductase activity of eNOS reside within the ACE.

Although present in a truncated form in nNOSs, the pentabasic motif (RRKRK) is perfectly conserved throughout all the available mammalian eNOS sequences. Our results suggest that the pentabasic motif is the structural feature which is responsible for the attenuated reductase function of eNOS. Removal of this motif results in an enzyme with maximal CaM-bound rates of NO synthesis, as well as cytochrome $c$ and ferricyanide reduction, that are comparable to that of nNOS. Support for this view comes from our finding that removal of this motif alleviates the residual inhibitory effects exerted on eNOS by the ACE, even in the presence of bound $\mathrm{Ca}^{2+} / \mathrm{CaM}$. A similar deletion (WRRKRKESS) using the same expression system, was reported to result in no significant change in the observed maximal activities and a slight decrease in the $\mathrm{EC}_{50}$ for $\mathrm{Ca}^{2+}$ (Chen \& Wu, 2000). The reason for these different findings is assumed to be due to the removal of the extra four amino acids. Indeed, we would assume that the length of the residual ACE in mutated eNOSs is very important if, as hypothesized, the functioning of the ACE involves multiple sites of interaction.

A role for the RRKRK motif in attenuation of electron flux through the reductase domain is supported by the ACE-derived peptide studies described in section I. Here, the most potent peptides were derived from the ACE of eNOS and contained the pentabasic motif. Potency increased as the pentabasic region was 
gradually lengthened to include the entire ACE sequence. Peptides derived from the ACE of nNOS were much less potent inhibitors of NOS activity. This suggests that, due to the presence of the RRKRK motif, the ACE of eNOS is a much better inhibitor of NOS activity than the ACE of nNOS.

We previously proposed the RRKRK motif as a site of interaction between the $\mathrm{ACE}$ and the surface of eNOS or bound CaM. We suggested a possible complementary binding site for this motif to be a "patch" of acidic residues in the oxygenase domain of cNOSs. Given the accelerated rate of CaM dissociation, relative to wild type eNOS, that we observed in mutants lacking the RRKRK motif, an interaction between the RRKRK motif and acidic regions of CaM may be more likely. From our ACE-derived peptide studies, mutation of the RRKRK motif would be expected to decrease the rate of CaM-dissociation from eNOS. Our results demonstrate the reverse situation, the lack of an RRKRK motif accelerates CaMdissociation. One possible interpretation is that the RRKRK motif is involved in ACE-CaM interactions (or interactions with regions flanking the CaM-binding site) that are not required for activation, but rather prevent "optimal" (nNOS-like) CaMbinding. This may limit the extent of the CaM-induced conformational changes and account for the reduced activity of eNOS, compared to nNOS.

The role of the conserved N-terminal region of the ACE of cNOSs was not investigated by us, but was hypothesized to dictate the relative $\mathrm{Ca}^{2+}$-dependence, similar for both eNOS and nNOS. Deletion of a similar region of eNOS (QHKSYKIR) (Chen \& Wu, 2000), resulted in a decrease in the $\mathrm{EC}_{30}$ for $\mathrm{Ca}^{2+}$ required for activity without a dramatic effect on rate of NO synthesis, compared to 
wild type (97 \pm 24 and $138 \pm 21 \mathrm{nmol} / \mathrm{min} / \mathrm{mg}$, respectively) Although a significant portion of this activity was $\mathrm{Ca}^{2+}$-independent $(18 \pm 6 \mathrm{nmol} / \mathrm{min} / \mathrm{mg})$, iNOS-like, highaffinity CaM-binding was not observed. Additionally, a much larger shift in $\mathrm{Ca}^{2+}$ dependence was observed when the entire (or entire minus eNOS ${ }_{607-613}$ ) ACE was deleted from eNOS (Chen \& Wu, 2000; Nishida \& Ortiz de Montellano, 1999).

The RRKRK motif may also provide a basis for the classification of $\mathrm{Ca}^{2+} / \mathrm{CaM}$-dependent NOSs of invertebrate origins, such as those expressed by Rhodnius prolixus (Yuda et al., 1996) and Drosophila melanogaster (Regulski \& Tully, 1995). As the RRKRK motif is conserved in all the currently available eNOS sequences, we can consider this as criteria for classification at the molecular level. In the same way that presence of an ACE within the FMN binding domain defines cNOSs, the presence of an RRKRK motif within the ACE defines cNOS classification to specify eNOSs. All of the invertebrate cNOS sequences available to date do not express this motif within their ACE regions (Fig. 3.6, panel B), classification of these proteins as nNOSs would therefore seem appropriate.

\subsubsection{Regulation of eNOS by Phosphorylation of a Conserved cAMP-dependent}

Protein Kinase Site ( $\operatorname{Ser}_{633}$ ) Within the Autoinhibitory Control Element.

Although eNOS is classified as $\mathrm{Ca}^{2+}$-dependent, other methods of regulation are inherent given the ability of certain stimuli to activate eNOS in a $\mathrm{Ca}^{2+}$ independent manner; such stimuli include estrogen (Caulin-Glaser et al., 1997), insulin (Zeng \& Quon, 1996), insulin-like growth factor (Tsukahara et al., 1994) and vascular shear-stress (Ayajiki et al., 1996; Kuchan \& Frangos, 1994). Given that a 
large part of the regulation of eNOS activity rests with displacement of the autoinhibitory peptide, it is conceivable that reversible, $\mathrm{Ca}^{2+}$-independent mechanisms have evolved to relieve the exerted inhibitory effects. To gain insight into the mechanisms involved in " $\mathrm{Ca}^{2+}$-independent" eNOS activity, we considered the well characterized CaM-dependent protein kinase II (Strack et al., 1997). In this CaMdependent system autophosphorylation of the autoinhibitory domain mediates $\mathrm{Ca}^{2+}$ independent activity. For eNOS, an attractive possibility would be that phosphorylation of the ACE can modify a site(s) of interaction, and hence relieve/reduce its imposed inhibitory actions.

The ACE of eNOS is very rich in Ser and Thr residues and sequence analysis identified a conserved consensus site for cAMP-dependent protein kinase (PKA) mediated phosphorylation $\left({ }^{R} /{ }_{K} / K X^{R} / T\right)$ at $\operatorname{Ser}_{635 / 636}$ (RKESS). We demonstrated $\operatorname{Ser}_{6,33}$, in addition to $\mathrm{Ser}_{494}$ within the CaM-binding domain, to be an in vitro target for PKAmediated phosphorylation. However, functional analysis of phosphorylated eNOS showed no significant effect on the maximal rate of NO synthesis, maximal reductase activities, or the $\mathrm{EC}_{50}$ of free $\mathrm{Ca}^{2+}$ required for enzyme activation. These findings are challenged by recent evidence suggesting a regulatory role for phosphorylation of this residue of eNOS by both PKA and cGMP-dependent kinase (PKG)(Butt et al., 2000). This group showed that eNOS was phosphorylated by both PKA and PKG in vitro and involved both Ser and Thr residues. Mass spectrometry identified phosphorylated residues corresponding to $\operatorname{Ser}_{1177}$ (analogous to $\operatorname{Ser}_{1179}$ in the bovine sequence), at the C-terminus and $\operatorname{Ser}_{633}$ (analogous to $\mathrm{Ser}_{635}$ in the bovine sequence) within the ACE. Functional characterization of in vitro phosphorylated eNOS showed a reproducible, partial activation (10-20\% of maximum), which was independent of $\mathrm{Ca}^{2+} / \mathrm{CaM}$. This 
also correlated with an approximate 4-fold increase in $\mathrm{V}_{\max }$ in the presence of bound $\mathrm{Ca}^{2+} / \mathrm{CaM}$. Additionally, PKA stimulation in intact endothelial cells increased both $\mathrm{Ca}^{2+} / \mathrm{CaM}$-independent and -dependent eNOS activity. However, more recent evidence would suggest that phosphorylation of $\mathrm{Ser}_{1177}$ in the C-terminus of eNOS was the preferred site of phosphorylation by PKA (Chen et al., 1999), and accounted for observed changes in phenotype that had been attributed by Butt et al to $\operatorname{Ser}_{635}$ phosphorylation. A mounting body of evidence now exists to support $\operatorname{Ser}_{1179}$ as the major, phosphorylation-dependent site of eNOS regulation from studies involving protein kinase B (PKA/Akt) (Dimmeler et al., 1998; Dimmeler et al., 2000; Dimmeler et al., 1999; Fisslthaler et al., 2000; Fulton et al., 1999; Luo et al., 2000; McCabe et al., 2000), this will be discussed in detail in chapter 4 .

In order to bypass complications of experimental interpretation owing to simultaneous phosphorylation of multiple sites, we assessed the possible regulation of eNOS by phosphorylation of $\operatorname{Ser}_{635}$ in a slightly different manner. We produced a mutant eNOS in which this Ser residue was exchanged for a Glu residue, the charge pattern of which can emulate the effects of a phosphoserine. However, these proteins failed to support a role for phosphorylation of the ACE in the regulation of enzyme activity. We must conclude that the regulatory effects attributed to phosphorylation of this residue (Butt et al., 2000), were in fact due to the phosphorylation of $\operatorname{Ser}_{1179}$ within the C-terminus of eNOS. 


\section{CHAPTER 4}

\section{THE C-TERMINUS OF ENDOTHELIAL NITRIC OXIDE SYNTHASE}

\section{IMPEDES CALCIUM/CALMODULIN-INDUCED ENZYME ACTIVATION}

\section{AND SERVES AS A SECOND AUTOINHIBITORY CONTROL ELEMENT}

\subsection{Introduction}

In chapter 3, we previously demonstrated that the FMN binding subdomain of $\mathrm{Ca}^{2+}$-dependent isoforms of nitric oxide synthase (cNOS) contain a 45 amino acid insertion that functions as an autoinhibitory control element (ACE)(Salerno et al., 1997). Limited proteolysis studies revealed that the ACE is physically displaced upon CaM-binding to cNOS and that synthetic ACE-derived peptides inhibit both CaM binding and NOS activation. Homology-based molecular modeling provided structural predictions consistent with an interaction between the $\mathrm{ACE}$ and bound $\mathrm{CaM}$ on cNOS. A model was posed whereby the $\mathrm{ACE}$ reversibly docks with a site on cNOS that impedes CaM binding and hence, enzyme activity. Binding of CaM to cNOS was postulated to displace the ACE by virtue of domain overlap, thereby eliciting enzyme activation. Support for this prediction was subsequently provided by demonstrations that deletion of the entire ACE from eNOS(Chen \& Wu, 2000; Nishida \& Ortiz de Montellano, 1999) or nNOS(Daff et al., 1999; Montgomery et al., 2000) resulted in a marked reduction in the $\mathrm{Ca}^{2+}$ concentration required for activation of NO synthesis. Deletion of the ACE from eNOS also caused an increase in the maximal rate of NO synthesis, to a level comparable with that of wildtype nNOS and iNOS(Nishida \& Ortiz de Montellano, 1999). The mechanism by which ACE deletion 
enhances NO production by eNOS must explain a specific acceleration in electron flux through the reductase domain.

Recognition sites on cNOSs that interact with the ACE to modulate activity remain to be elucidated. That such sites reside within the reductase domain is indicated by the finding that $\mathrm{Ca}^{2+} / \mathrm{CaM}$-dependent control of electron flux remains intact in isolated eNOS- and nNOS-derived reductase domains (Abu-Soud \& Stuehr, 1993; Abu-Soud et al., 1994). Despite the lack of a solved structure for any NOS reductase domain, 3-D homology-based models of NOS reductase subdomains can be developed considering many related proteins with defined high-resolution structures (Salerno et al., 1997). Only two regions of eNOS can not be reliably modeled in this manner due to the lack of suitable structural homologs. These completely unknown structural elements are the aforementioned 45 amino acid ACE peptide that resides in the FMN-binding subdomain and a C-terminal peptide (27-42 amino acids, depending on NOS isoform) that is not found in any related FAD-containing flavoprotein and is partially sequence-conserved within NOS isoforms.

The C-terminal peptide is not needed for enymatic activity, inasmuch as NO synthesis is not attenuated by truncation of this region in iNOS (Xie et al., 1994). Nonetheless, the C-terminal peptide could conceivably function in regulation of enzyme activity. Recent studies have shown that endothelial shear-stress results in phosphorylation of a conserved serine within this C-terminal peptide of eNOS (Ser 1179 in bovine eNOS) that causes attenuated $\mathrm{Ca}^{2+}$-dependence and enhanced catalytic activity in vivo (Chen et al., 1999; Dimmeler et al., 1999; Fulton et al., 1999). We describe here the expression and characterization of a truncated bovine eNOS mutant 
lacking Ser 1179 and the subsequent 26 C-terminal amino acids. Herein we present evidence supporting a key functional role for this $\mathrm{C}$-terminal extension in determining the $\mathrm{Ca}^{2+}$-dependence for activation by calmodulin and in controlling the rate of eNOS catalysis. 


\subsection{Results}

\subsubsection{Identification and Characterization of an Autoinhibitory Control}

\section{Element Within the C-terminal Extension of eNOS}

Despite $>40 \%$ homology between the C-terminal regions of rat cytochrome P450 reductase (CPR) and NOS isoforms, alignments revealed that the C-termini of all NOSs extend beyond the site of CPR termination (Fig. 4.1 panel A). The fact that CPR is functionally homologous to the NOS reductase domain, including binding sites for NADPH, FAD and FMN, implies that the C-terminal extension in NOSs is not required for catalytic function. Indeed, it has been demonstrated for murine iNOS that all 31 amino acids C-terminal to regions homologous to CPR, can be deleted from the C-terminus without loss of activity (Xie et al., 1994). Any further truncation of iNOS results in a loss of activity. Despite being surplus to requirements for NOS catalysis, significant identity in the C-termini of each individual isoform, and general conservation among all isoforms, suggests functional importance (Fig. 4.1, panel B).

However, as for the previously described FMN-insertion, the lack of crystalstructures for the reductase domain of any of the NOS isoforms hampered the formation of a structurally-based hypothesis for the function of the C-terminal extension. Again, we turned to homology to gain insight into the possible regulatory function of the C-terminus of eNOS and we considered the high-resolution crystal structure of rat CPR (Wang et al., 1997). Although this exercise provides no direct structural information about the C-terminus of eNOS, the relative positioning of the C-terminus in CPR is telling. Notably, the C-terminus of CPR curls back towards the 
main body of the enzyme and ends in a region proximal to the binding sites for both NADPH and FAD (see Fig. 4.2). The penultimate residue of CPR is Trp, which lies at the interface between bound NADPH and FAD. In this setting the aromatic ring of Trp is coplanar with the isoalloxazine ring of FAD and positioned in a manner in which the $\pi$-cloud of electrons could reasonably modulate electron transfer between NADPH and FAD. All NOSs contain the conservative aromatic substitution of Phe in place of Trp in CPR (see Fig. 4.1). Given this arrangement, one can readily imagine that interactions of the $\mathrm{C}$-terminus could cause subtle reorientation of this aromatic residue, and hence serve to regulate electron flux through the reductase domain. Importance of this Phe in NOS catalysis has already been demonstrated for iNOS; while activity is not diminished by removal of all subsequent $\mathrm{C}$-terminal amino acids, deletion of this Phe results in $71 \%$ loss in maximal activity (Xie et al., 1994).

To investigate the possible regulatory function for the C-terminal extension of eNOS, we expressed and purified a mutant eNOS truncated at the C-terminus. Introduction of a stop codon in lieu of $\mathrm{Ser}_{1179}$, a residue that has previously been implicated as a site of in vivo regulation by the kinase Akt/PKB (Dimmeler et al., 1999; Fulton et al., 1999), results in C $\Delta 27$, a truncated eNOS protein that is lacking 27 of the $42 \mathrm{C}$-terminal amino acid residues that extend beyond the termination of CPR (see Fig. 4.1). 
WW WW W

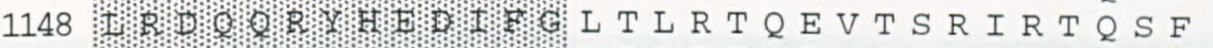

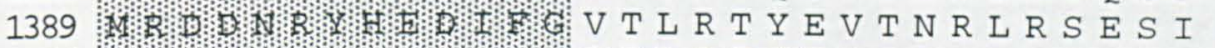
1384

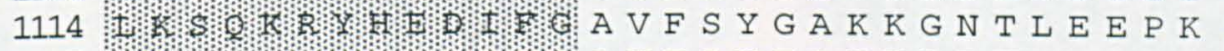

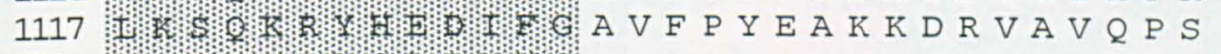

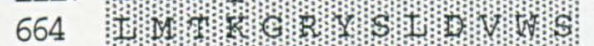

1 The

Bovine eNOS Human eNOS human nNOS rat nNOS Rat iNOS human iNOS cpr450 protein

1180 S L Q E R H R G A V P A F D P P P D T P G P C27 eNOS

1178 S L Q E R Q L R G A V P W A F P P G S T N S P

Bovine eNOS

1419 A F I E E S K K T DEV F S

1414 A F I E E S K K D A D E F S S

1144 G T R L

1147 S L E M S A L

676

28

Human eNOS

human nNOS

rat nNOS

Rat iNOS

human iNOS

cpr450 protein

C27 ENOS

B

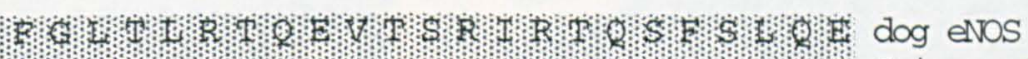

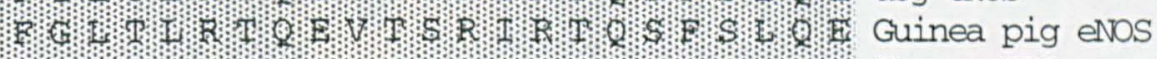

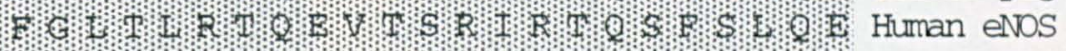

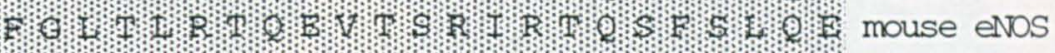

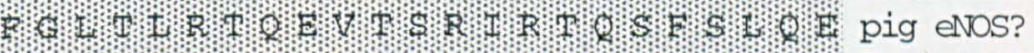

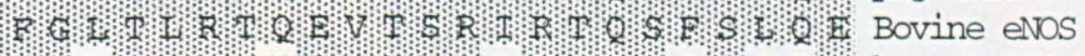

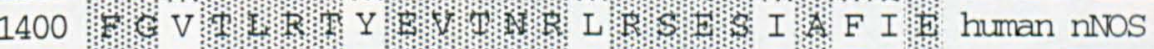

1395 V V W

1395 V V

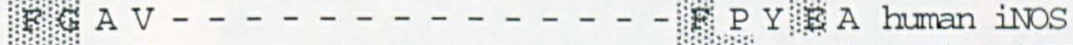

28

1185 W

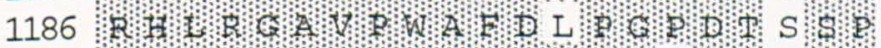

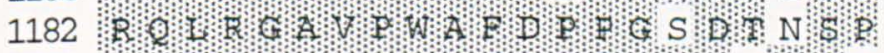

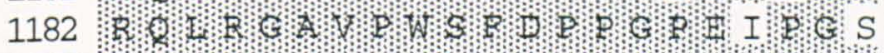
1185 1. 1184 KW 1423 E S K Y D

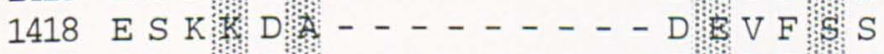

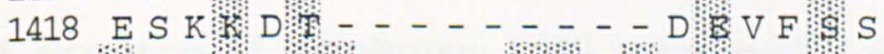
1137 K D W V K 1131 K K S S L E - - - D K K
C27 eNOS dog eNOS Guinea pig eNOS Human eNOS mouse eNOS pig eNOS? Bovine eNOS human nNOS rat nNOS Mouse nNOS human iNOS murine inOS

Fig. 4.1. Sequence alignment of various NOS isoforms and cytochrome P450 reductase (CPR). Alignment was performed by the Clustal method using DNA Star Megalign, a program in the Lasergene suite of molecular biology tools. Panel $\mathrm{A}$ : Comparison of $\mathrm{CPR}$ with representative members of the three NOS isoforms showing the C-termini (shaded regions are conserved between CPR and NOSs). Panel B: Conservation of regions of the C-terminal extension between NOS isoforms (shaded regions are conserved). 


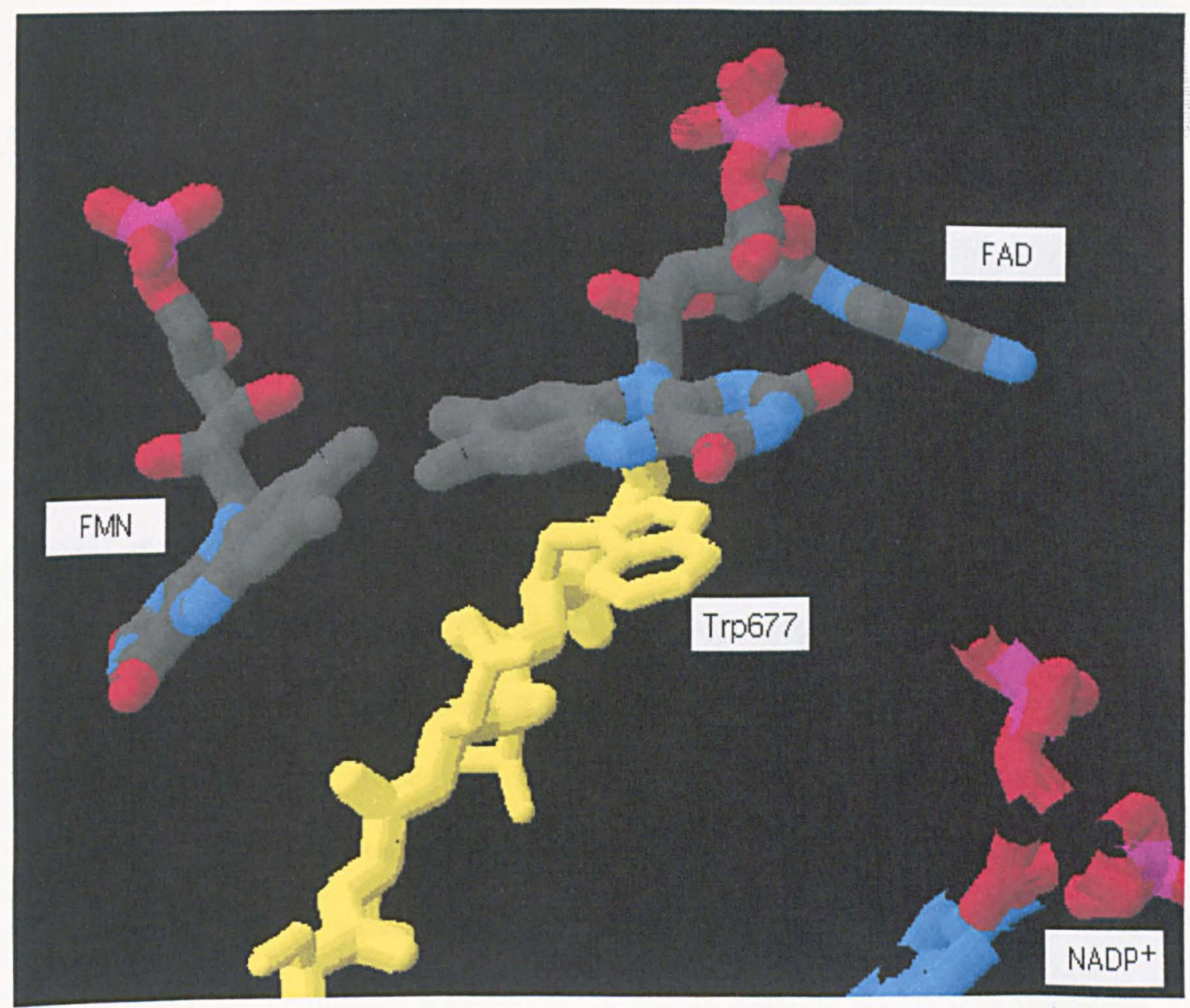

Fig. 4.2. Structure of cytochrome P450 reductase (Wang, et al 1997) showing only the positioning of the C-terminal residues (yellow) in relation to the bound cofactors, FAD, FMN and NADP ${ }^{+}$. Note that the penultimate Trp residue stacks with the FAD isoalloxazine ring at the juncture between the three cofactors. 


\subsubsection{Expression and purification.}

A two-step purification involving sequential affinity chromatography steps on ADP-sepharose and CaM-sepharose resins resulted in $>90 \%$ homogeneity for both wildtype and C $\Delta 27$ eNOS, as estimated from Coomassie-stained SDS/PAGE (Fig. 4.3). After several unsuccessful attempts at purification of expressed C $\Delta 27$ eNOS using the established protocol for wildtype eNOS, we investigated the reasons for our failure. After establishing the validity of expression by comparing the Triton X-100 lysates of IPTG-induced vs. non-induced BL21 $E$. coli cells by SDS-PAGE, we next investigated the location of the desired protein. Surprisingly, we found the protein was localized to the particulate fraction of centrifuged lysates and, as only the soluble fraction was used for subsequent steps, was lost during processing. However, denaturing methods (Triton X-100, organic extraction) did not yield viable eNOS. Such methods have been used to purify other proteins, but due to eNOS being a large, complex enzyme containing a variety of cofactors, such denaturation/renaturation methods are unsuitable. The inclusion of the relatively gentle detergent CHAPS had previously been used to remove acylated-wildtype eNOS from anchorage sites in mammalian cell membranes (Garvey et al., 1994; Liu \& Sessa, 1994; Pollock et al., 1992). Hence, we tried this approach in our E. coli-based expression system. Extraction of CA27 eNOS (but not wildtype eNOS) from bacterial pellets was significantly improved by the inclusion of CHAPS $(20 \mathrm{mM})$ in the bacterial lysis buffer. We assume this to be due to the predicted exposure of hydrophobic residues in the C-terminus of $\mathrm{C} \Delta 27 \mathrm{eNOS}$, which lead to a membrane associated protein. Overall protein yields ranged from $2-10 \mathrm{mg}$ purified protein per liter of bacterial culture. 
Spectrophotometry demonstrated that the CO-bound spectra of dithionitereduced $\mathrm{C} \Delta 27$ and wt eNOS were indistinguishable (Fig. 4.4), indicating that Cterminal truncation does not compromise heme-coordination. Accordingly, spectral assessment of heme-chromophore was used to quantify eNOS protein mass. Additionally, truncation of the C-terminus did not attenuate the ability of $\mathrm{C} \Delta 27 \mathrm{eNOS}$ to form homodimeric complexes (Fig. 4.5).

Now that we had expressed and purified functional $\mathrm{C} \Delta 27$ eNOS, we investigated the effects of $\mathrm{C}$-terminal truncation by characterization of the protein with relation to eNOS catalytic function and regulation by $\mathrm{Ca}^{2+} / \mathrm{CaM}$.

\subsubsection{Catalytic activities of $\mathrm{C} \Delta 27$ and wildtype eNOS}

Three catalytic activities of eNOS were analyzed and contrasted for wildtype and $C \Delta 27$ enzymes (Figs. 4.6 and 4.7). These distinct activities were determined in the absence and presence of bound-CaM $(250 \mathrm{nM})$ and indicate maximal electron flux to heme (NO synthesis, Fig. 4.6), FAD (cytochrome c reduction, Fig. 4.7 panel A) and FMN (ferricyanide reduction, Fig. 4.7 panel B) (Adak et al., 1999).

As shown in Fig 4.6, neither wildtype nor C $\Delta 27$ eNOS supported detectable levels of NO synthesis in the absence of added CaM (although our laboratory has previously detected CaM-independent basal NO synthesis by eNOS using the much more sensitive $\left[{ }^{3} \mathrm{H}\right]$-citrulline assay). Truncation of the $\mathrm{C}$-terminus of eNOS resulted in a 2-fold increase in maximal CaM-induced NO synthesis, compared to wildtype enzyme (from $152 \pm 14$ to $303 \pm 4 \mathrm{nmol} / \mathrm{min} / \mathrm{mg}$ ). 
Given that the rate-limiting step for NO synthesis by CaM-bound eNOS appears to reside in the reductase domain (Nishida \& Ortiz de Montellano, 1999; Nishida \& Ortiz de Montellano, 1998), we monitored the rate that artificial electron acceptors could be reduced by electrons derived from reductase domain flavins of wildtype and $\mathrm{C} \Delta 27$ eNOS. Although significant basal cytochrome $\mathrm{c}$ and ferricyanide reduction was observed with both $\mathrm{C} \Delta 27$ and wildtype eNOS; basal activity was 3-4 fold greater with $\mathrm{C} \Delta 27$ (Fig. 4.7, panels $\mathrm{A}$ and B, respectively). In both cases, activity was accelerated 2-3 fold over basal after addition of excess CaM. Interestingly, the basal reductase activities of $\mathrm{C} \Delta 27$ were greater than the CaMstimulated activities of wildtype eNOS. These findings reveal that truncation of eNOS's C-terminus markedly accelerates both basal and maximal CaM-induced electron flux to FAD and FMN.

\subsubsection{Effect of a C-terminal extension-derived peptide on eNOS activity}

As the use of synthetic peptides had provided insights into the function of the ACE present within the FMN-binding domain, we assessed the effect of a peptide $\left(\right.$ eNOS $\left._{1179-1205}\right)$ derived from the C-terminal extension on eNOS activity (corresponding to the 27 amino acids deleted in C $\Delta 27$ eNOS). However, unlike peptides derived from the FMN domain-ACE, eNOS ${ }_{1179-1205}$ was only weakly inhibitory, attenuating NO synthesis, $20 \%$ with $100 \mu \mathrm{M}$ of peptide (Fig. 4.8). Inhibition of $\mathrm{C} \Delta 27$ eNOS by $\mathrm{eNOS}_{1179 \cdot 1205}$ followed a similar pattern to that observed for wildtype eNOS. Due to this lack of inhibition, the effects of this peptide on $\left[{ }^{[25} \mathrm{I}\right]-$ CaM binding were not assessed.

We also assessed the effect of this peptide on the reductase domain of eNOS; a dose-response relationships were established for both CaM-dependent and 
-independent reductase activities. For wildtype eNOS, both CaM-dependent and -independent cytochrome $\mathrm{c}$ reduction (FMN-mediated) were unaffected by NOS $_{1179}$. ${ }_{1205}$ at all concentrations tested ( $\leq 100 \mu \mathrm{M}$, Fig. 4.9, panel A). Truncation of the Cterminus did not make eNOS more susceptible to inhibition of cytochrome c reductase activity (Fig. 4.9, panel B). Similarly, no effect was observed on ferricyanide reductase activity for either wildtype or $\mathrm{C} \Delta 27 \mathrm{eNOS}$ (Fig. 4.10, panels A and B).

Peptide studies outlined above would suggest that the C-terminal extension is not autoinhibitory, although removal of this region by mutation results in increased catalytic rates. Therefore, the phenotype of $\mathrm{C} \Delta 27$ eNOS supports the proposed autoinhibitory role of the C-terminal extension, hence referred to as autoinhibitory control element 2 (ACE-2), in contrast with the established ACE-1 within the FMNbinding domain. However, although truncation of ACE-2 enhanced the basal, CaMfree reductase activities of eNOS to a level greater than that of CaM-bound wildtype eNOS, additional CaM-induced conformational changes were required for this increase to manifest as NO production. CaM binding increases both the intra-domain (NADPH $\rightarrow$ FAD $\rightarrow$ FMN), and inter-domain (reductase domain $\rightarrow$ oxygenase domain, FMN $\rightarrow$ heme) electron flux through cNOSs (Abu-Soud et al., 1994). We assume that these additional $\mathrm{CaM}$-induced conformational changes are required to provide the functional FMN-heme alignment required for NO synthesis. We therefore investigated the effects of truncation of ACE- 2 on the regulation of eNOS by $\mathrm{Ca}^{2+} / \mathrm{CaM}$. 


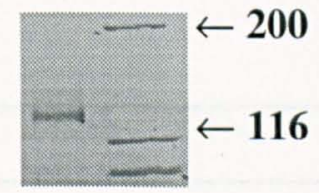

Fig. 4.3. SDS-polyacrylamide gel electrophoresis of purified recombinant C $\Delta 27$ eNOS. Recombinant $\mathrm{C} \Delta 27$ eNOS eNOS protein was purified to $>90 \%$ homogeneity and showed approximate molecular weight of $135 \mathrm{kDa}$. 

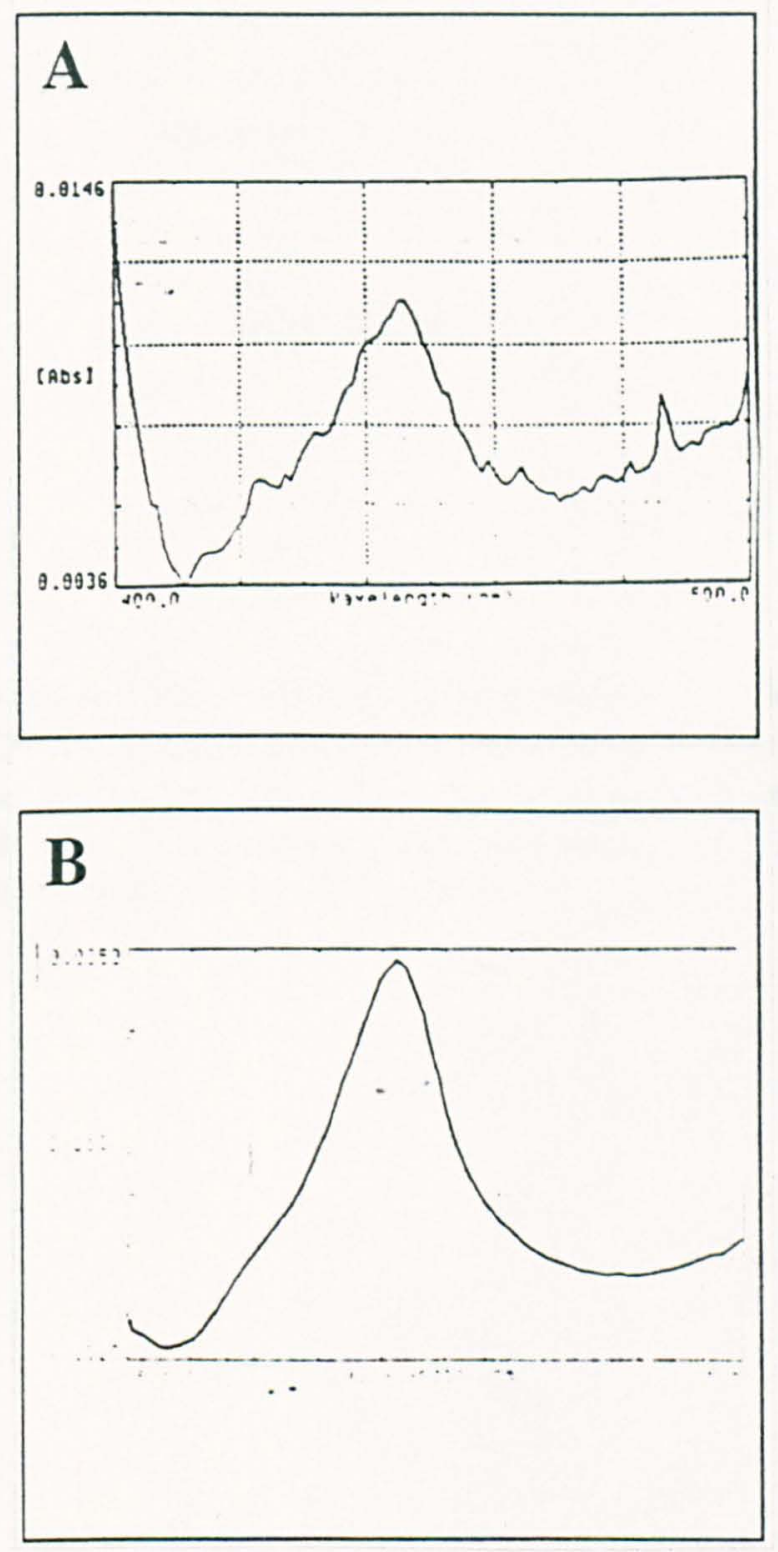

Fig. 4.4. P450 spectra of wildtype and $\mathrm{C} \Delta 27$ eNOS. The CO-bound, dithionite reduced spectra of wild type (panel A) and $\mathrm{C} \Delta 27$ eNOS (panel B) were obtained as described in methods. 
wildtype $\quad \mathrm{C} \Delta 27$

$$
\begin{aligned}
& 200 \rightarrow \\
& 130 \rightarrow
\end{aligned}
$$

Fig. 4.5. Low-temperature SDS-polyacrylamide gel electrophoresis of wildtype and $\mathbf{C} \Delta \mathbf{2 7}$ eNOS. Samples were prepared in SDS gel loading buffer as normal, but were not boiled. This allows the NOS homodimer to remain intact during electrophoresis. The apparent molecular weights of $>200 \mathrm{kDa}$ indicates the formation of stable dimers by $\mathrm{C} \Delta 27 \mathrm{eNOS}$. 


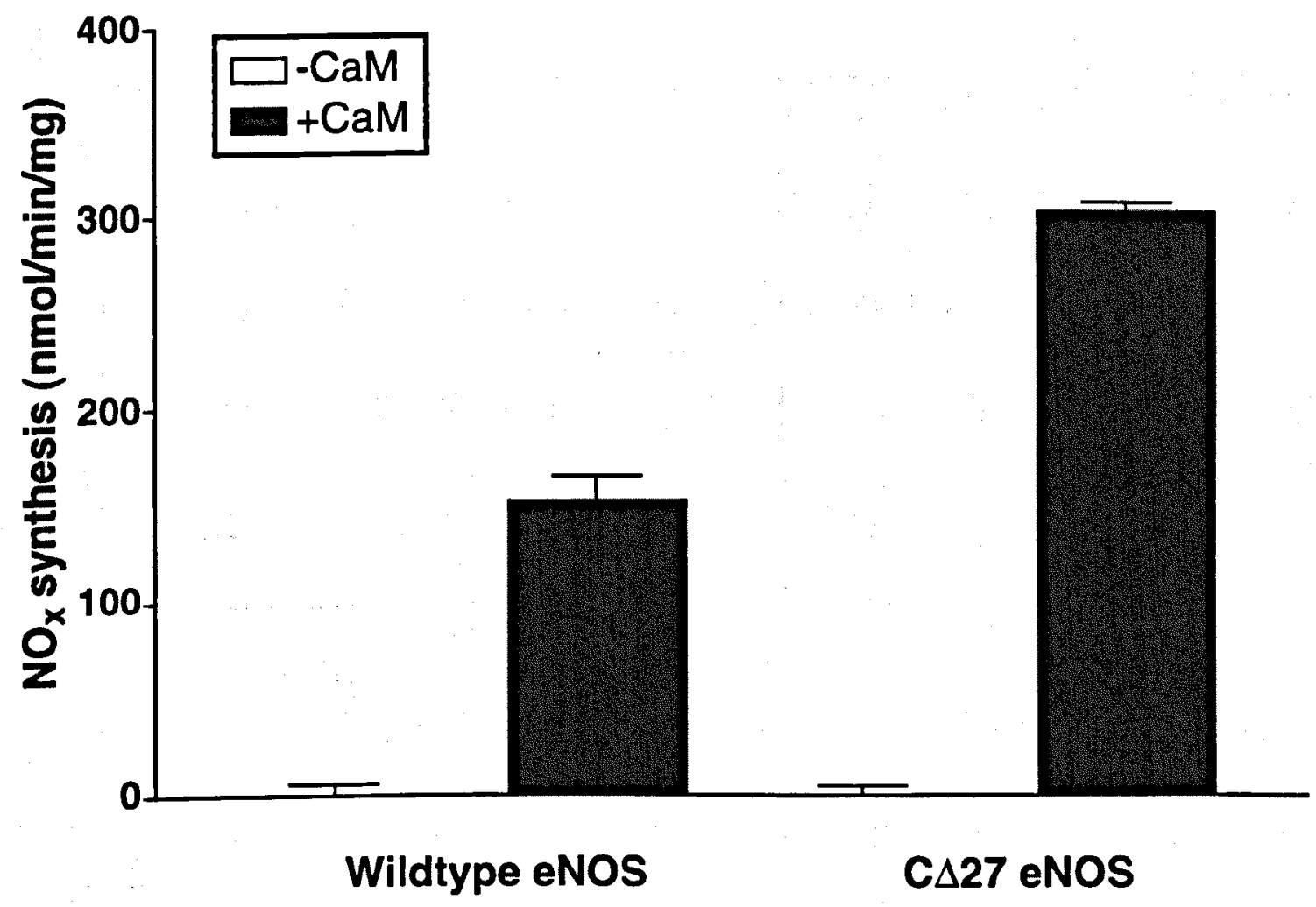

Fig. 4.6. Rates of NO synthesis by wildtype and C $\Delta 27$ eNOS. All experiments were carried out using 10 pmoles of NOS, $250 \mathrm{nM} \mathrm{CaM}$ and optimal $\mathrm{Ca}^{2+}$ concentrations $(100 \mu \mathrm{M})$. CaM-free measurements of activity were obtained in the presence of $5 \mathrm{mM}$ EGTA and rates are derived from the mean of two separate preparations of each enzyme, assayed in triplicate. 

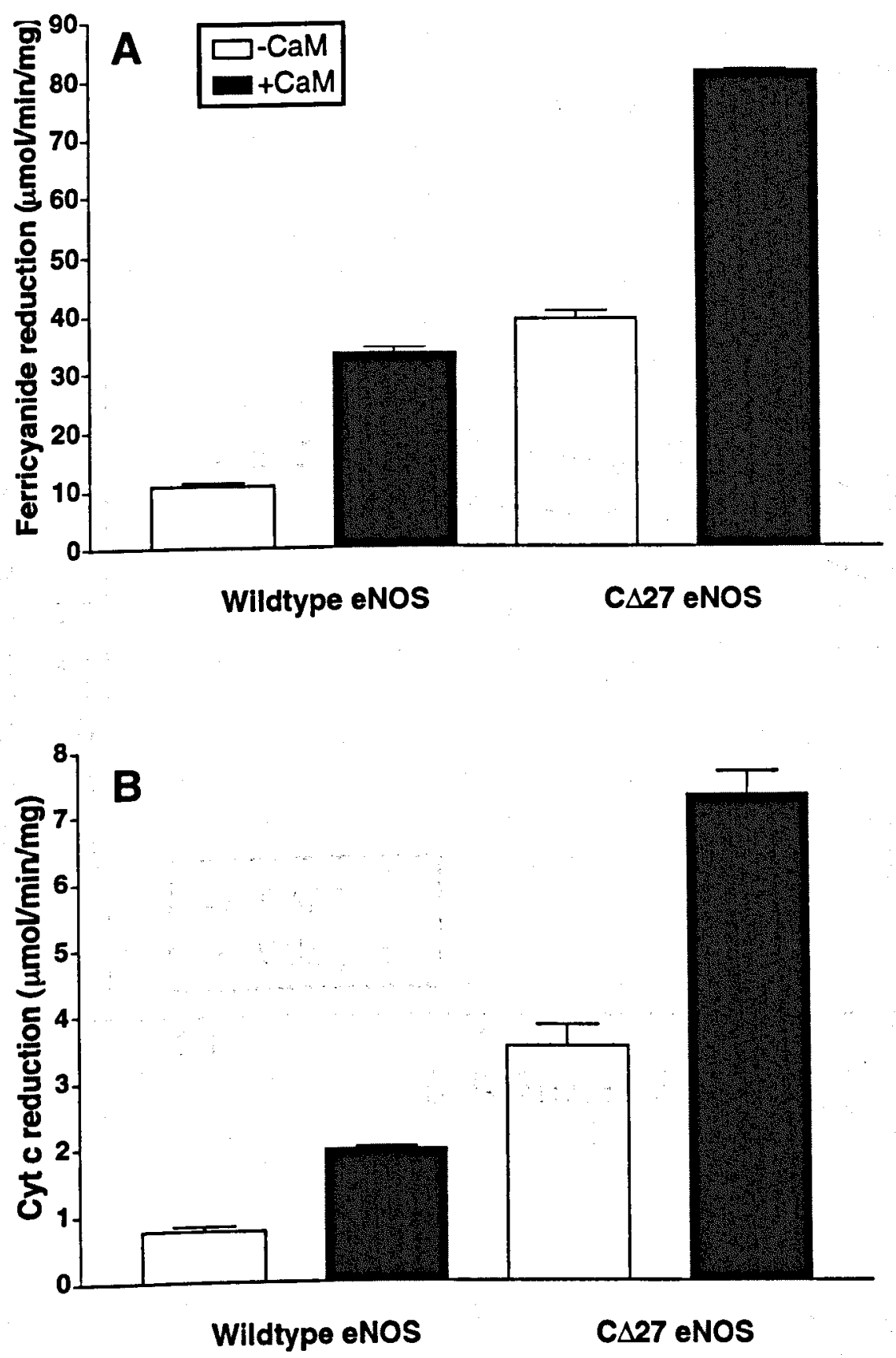

Fig. 4.7. Reductase activities of wildtype and $C \Delta 27$ eNOS. All experiments were carried out using $250 \mathrm{nM} \mathrm{CaM}$ and optimal calcium concentrations $(100 \mu \mathrm{M})$. Amounts of NOS added were 1 pmole and 0.1 pmole per well for cytochrome $\mathrm{c}$ reduction (Panel A) and ferricyanide reduction (Panel B), respectively. CaM-free measurements of activity were obtained in the presence of $5 \mathrm{mM}$ EGTA and rates are derived from the mean of two separate preparations of each enzyme, assayed in triplicate. 


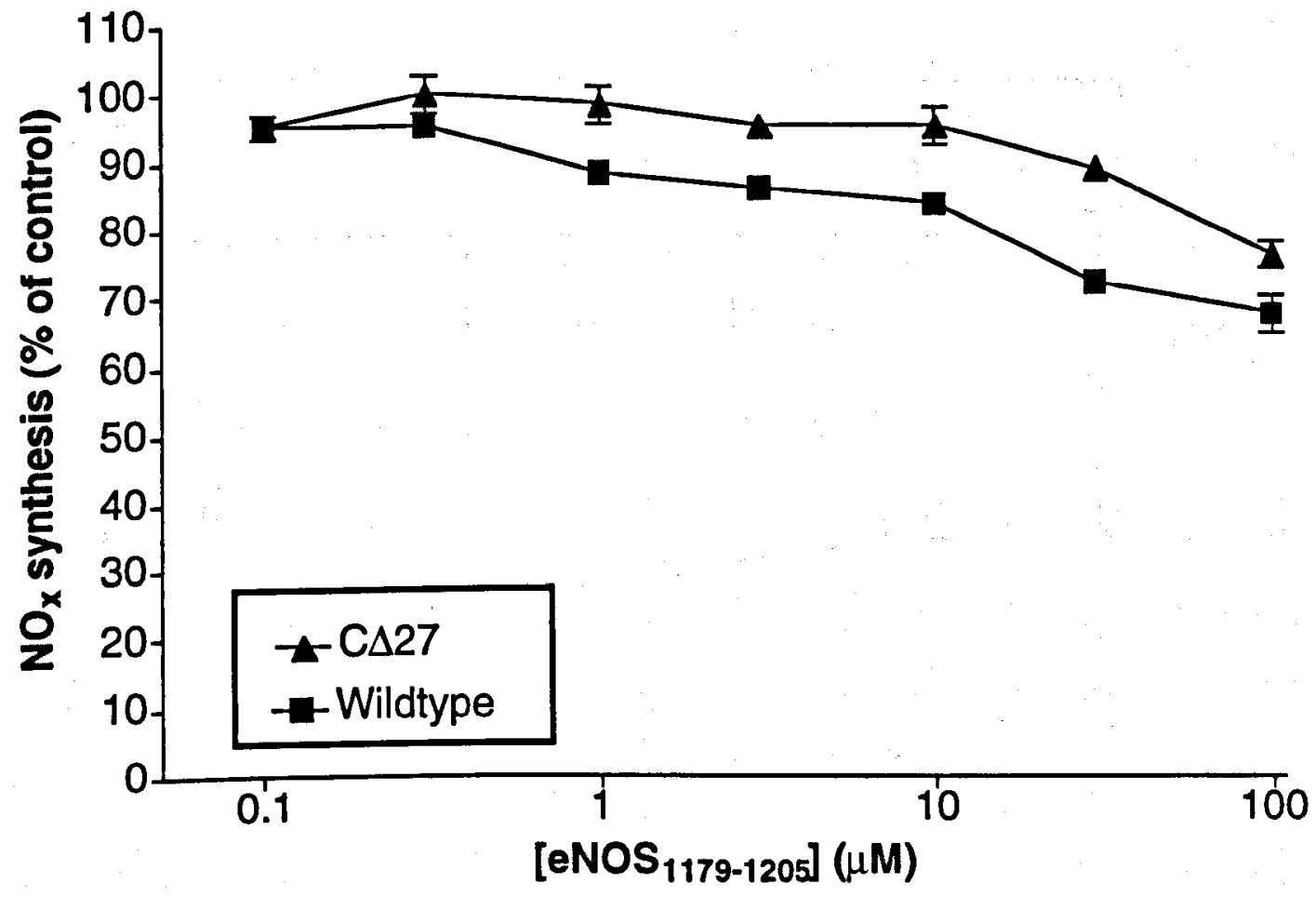

Fig. 4.8. Effect of $\mathrm{eNOS}_{1179-1205}$ on rate of NO synthesis of wildtype and CA27 eNOS. NO synthesis was assessed using the Griess assay as described in Methods in the presence of 10 pmole NOS, $100 \mathrm{nM} \mathrm{CaM}$ and optimal levels of $\mathrm{Ca}^{2+}(100 \mu \mathrm{M})$. CaM-independent activity was assessed in the presence of 5 mM EGTA. All values represent the mean of triplicate determination \pm SEM. 

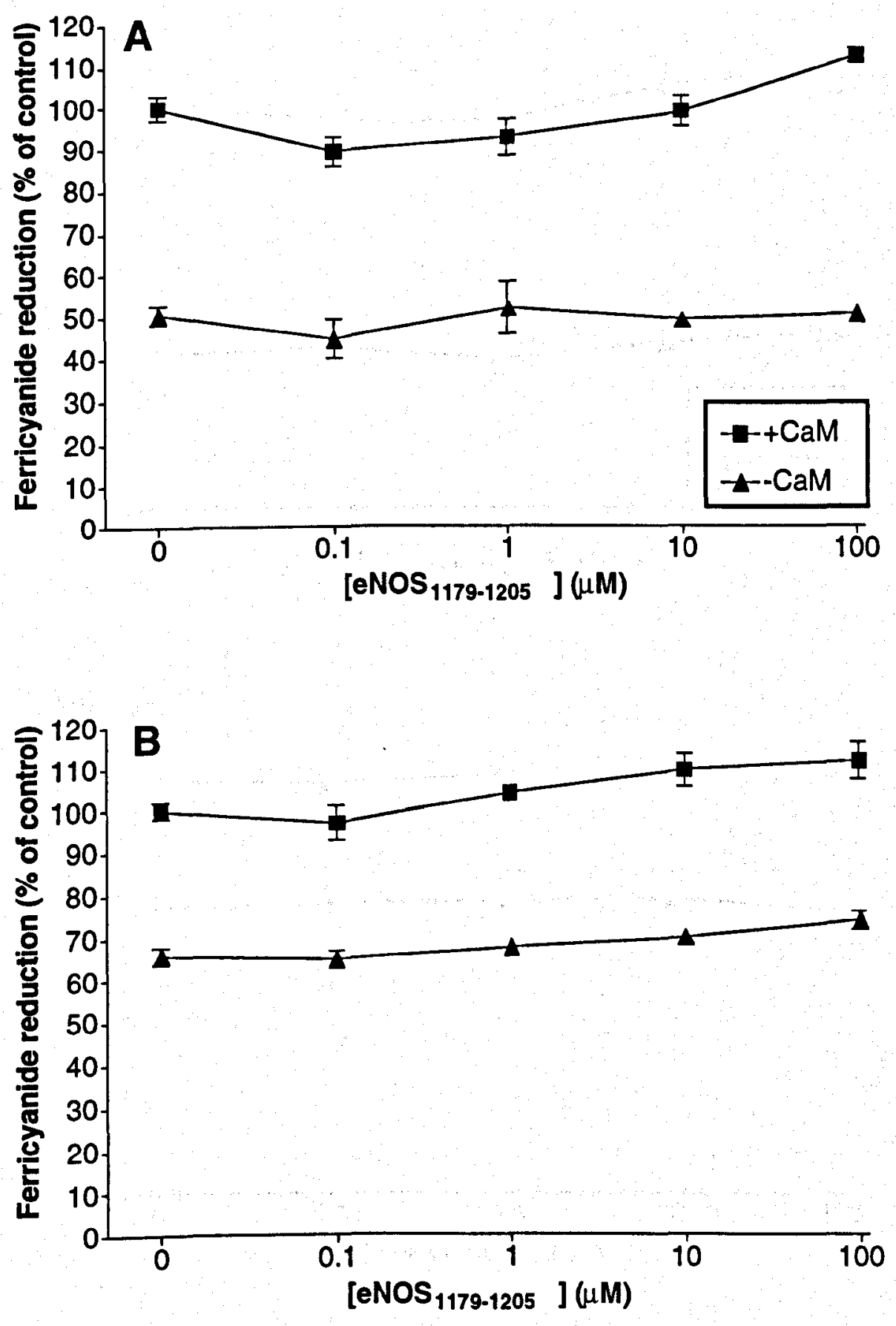

Fig. 4.9. Effect of eNOS ${ }_{1179-1205}$ on ferricyanide reductase activity of wildtype and C $\Delta 27$ eNOS. Ferricyanide reduction was assessed as described in the Methods in the presence of 0.1 pmole NOS, $250 \mathrm{nM} \mathrm{CaM}$ and optimal levels of $\mathrm{Ca}^{2+}(100 \mu \mathrm{M})$. CaMindependent activity was assessed in the presence of 5 mM EGTA. Results are shown for both wildtype (Panel A) and C $\Delta 27$ eNOS (Panel B). 

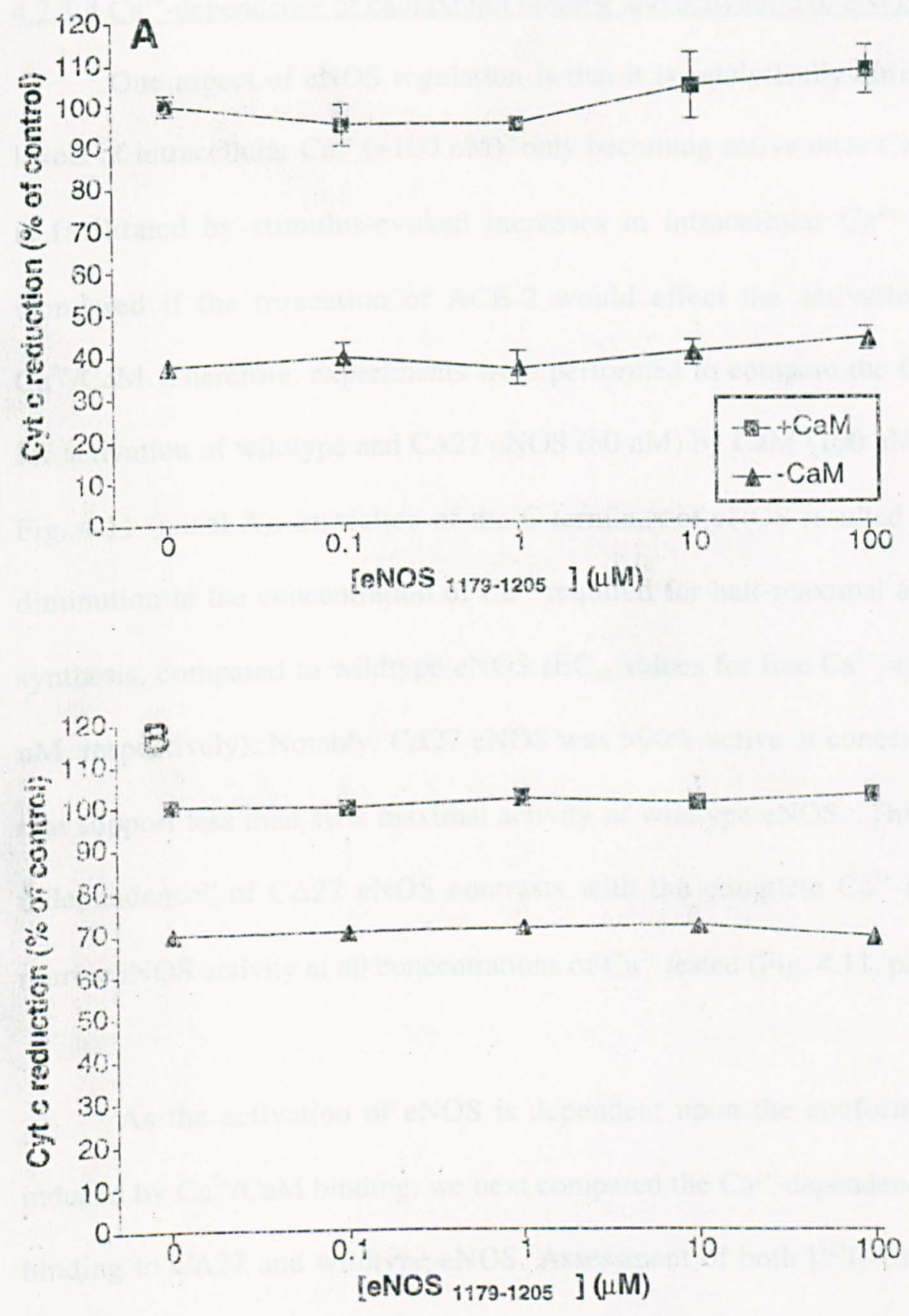

Fig. 4.10. Effect of eNOS ${ }_{1179.1205}$ on cytochrome c reductase activity of wilditype and $\mathrm{C} \Delta 27 \mathrm{eNOS}$. Cytochrome $\mathrm{c}$ reduction was assessed as described in methods in the presence of 1 pmole NOS, $250 \mathrm{nM} \mathrm{CaM}$ and optimal levels of $\mathrm{Ca}^{2+}(100 \mu \mathrm{M})$. CaM-independent activity was assessed in the presence of 5 mivi EGTA. Results are shown for both wildtype (Panel A) and CA27 eNOS (Panel B). 


\subsubsection{4 $\mathrm{Ca}^{2+}$-dependence of calmodulin binding and activation of eNOS}

One aspect of eNOS regulation is that it is catalytically quiescent at resting levels of intracellular $\mathrm{Ca}^{2+}(\approx 100 \mathrm{nM})$, only becoming active once $\mathrm{Ca}^{2+} / \mathrm{CaM}$ binding is facilitated by stimulus-evoked increases in intracellular $\mathrm{Ca}^{2+}(\leq 1 \mu \mathrm{M})$. We wondered if the truncation of ACE-2 would affect the activation of eNOS by $\mathrm{Ca}^{2+} / \mathrm{CaM}$. Therefore, experiments were performed to compare the $\mathrm{Ca}^{2+}$-dependence for activation of wildtype and C $\Delta 27 \mathrm{eNOS}(80 \mathrm{nM})$ by CaM $(100 \mathrm{nM})$. As shown in Fig. 4.11 (panel A), truncation of the C-terminus of eNOS resulted in a 5 to 6-fold diminution in the concentration of $\mathrm{Ca}^{2+}$ required for half-maximal activation of NO synthesis, compared to wildtype eNOS $\left(\mathrm{EC}_{50}\right.$ values for free $\mathrm{Ca}^{2+}=83 \mathrm{nM}$ and 461 $\mathrm{nM}$, respectively). Notably, $\mathrm{C} \Delta 27$ eNOS was $>90 \%$ active at concentrations of $\mathrm{Ca}^{2+}$ that support less than $10 \%$ maximal activity of wildtype eNOS. This relative " $\mathrm{Ca}^{2+}$ independence" of $\mathrm{C} \Delta 27$ eNOS contrasts with the complete $\mathrm{Ca}^{2+}$-independence of murine iNOS activity at all concentrations of $\mathrm{Ca}^{2+}$ tested (Fig. 4.11, panel A).

As the activation of eNOS is dependent upon the conformational changes induced by $\mathrm{Ca}^{2+} / \mathrm{CaM}$ binding, we next compared the $\mathrm{Ca}^{2+}$-dependence for $\left[{ }^{125} \mathrm{I}\right]-\mathrm{CaM}$ binding to $\mathrm{C} \Delta 27$ and wildtype eNOS. Assessment of both [ $\left.{ }^{125} \mathrm{I}\right]-\mathrm{CaM}$ binding (Fig.

4.11, panel B) and eNOS activity (Fig. 4.11, panel A) were performed using an identical array of $\mathrm{Ca}^{2+}$ buffer solutions, permitting direct comparison of observed $\mathrm{Ca}^{2+}$-dependencies. Despite the substantial difference between $\mathrm{EC}_{50}$ values for $\mathrm{Ca}^{2+}$ observed for activity of $\mathrm{C} \Delta 27$ and wildtype eNOS, curves reflecting the $\mathrm{Ca}^{2+}$ dependence for binding of $\left.{ }^{[25} \mathrm{I}\right]-\mathrm{CaM}$ exhibited a much smaller difference between wildtype and $\mathrm{C} \Delta 27$ eNOSs. For obvious reasons, $\left[{ }^{125} \mathrm{I}\right]-\mathrm{CaM}$ binding experiments were impossible to perform with iNOS. 
These findings are reconciled by a fundamental difference between these enzymes: (discussed more fully in Part II) whereas wildtype eNOS binds $\left[{ }^{125} \mathrm{I}\right]-\mathrm{CaM}$ at $\mathrm{Ca}^{2+}$ concentrations that are significantly lower than that required for physiological enzyme activation ( $\approx 4$-fold difference in $\mathrm{EC}_{50}$ values), the $\mathrm{Ca}^{2+}$-dependence for $\left[{ }^{125} \mathrm{I}\right]-$ CaM binding and activation of $\mathrm{C} \Delta 27$ eNOS are indistinguishable at $\mathrm{Ca}^{2+}$ concentrations above those typically found in resting cells $(100 \mathrm{nM})($ Persechini \& Cronk, 1999). It is notable however, that the physiological level of $\mathrm{Ca}^{2+}$ needed for eNOS activation is likely to be highly complex, and influenced by numerous factors, including the concentration of $\mathrm{Mg}^{2+}$, caveolins, heat shock protein 90 (HSP90), CaM, and the ratio of CaM to NOS.

\subsubsection{Calmodulin binding affinity}

As the $\mathrm{Ca}^{2+}$-independent activity of iNOS is due to its remarkably highaffinity binding of CaM (Cho et al., 1992), we wondered whether an increased affinity for $\mathrm{CaM}$ contributes to the reduced $\mathrm{Ca}^{2+}$-dependence for activation of $\mathrm{C} \Delta 27$ compared to wildtype eNOS. To examine this possibility, we defined the concentration-dependence for $\mathrm{CaM}$ to both activate and bind to $\mathrm{C} \Delta 27$ and wildtype eNOS, in the presence of excess $\mathrm{Ca}^{2+}(100 \mu \mathrm{M})$. In contrast to predictions, activity assays showed that $\mathrm{C} \Delta 27$ eNOS displayed a slightly reduced affinity for CaM vs. wildtype eNOS $\left(\mathrm{EC}_{50}=35\right.$ and $23 \mathrm{nM}$, respectively; see Fig. 4.12, panel A).

Saturation analysis of $\left[{ }^{125} \mathrm{I}\right]-\mathrm{CaM}$ revealed similar and essentially identical affinities of $\mathrm{CaM}$ for $\mathrm{C} \Delta 27$ vs. wildtype eNOS $\left(\mathrm{EC}_{50}=13 \mathrm{nM}\right.$ and $14 \mathrm{nM}$, respectively; see Fig. 4.12, panel B). Based on estimated calculations of free [CaM], 
correcting for the component of added $\left[{ }^{125} \mathrm{I}\right]-\mathrm{CaM}$ that contributes to total binding, we calculate $K_{d}$ values for $C \Delta 27$ and wildtype eNOS of $3 \mathrm{nM}$ and $4 \mathrm{nM}$, respectively. Such calculations were not possible for assays of NOS activity, as a direct measurement of bound-CaM was unavailable.

As a further analysis of the affinity for CaM binding of wildtype and $C \Delta 27$ eNOS, we assessed the rate of dissociation of eNOS-CaM complexes that had been formed at maximal levels of $\mathrm{Ca}^{2+}$. Dissociation was initiated using the previously described experimental design to prevent the re-association of $\left[{ }^{125} \mathrm{I}\right]-\mathrm{CaM}-\mathrm{cNOS}$ complexes, following addition of a 3000-fold molar excess of unlabelled CaM. The rate of dissociation of $\mathrm{C} \Delta 27-\mathrm{CaM}$ complexes was not discernibly different from that exhibited by CaM complexes involving wildtype eNOS; indeed fitted curves were virtually superimposable (Fig. 4.13, panel A). Linear analysis of this data indicated half times for $\left[\mathrm{I}^{125}\right]-\mathrm{CaM}$ dissociation of 21.4 and $21.0 \mathrm{~min}$ for wildtype and $\mathrm{C} \Delta 27$ eNOS, respectively (Fig. 4.13, panel B).

Binding affinity is determined by two variables, association rate $\left(\mathrm{k}_{\mathrm{on}}\right)$ and dissociation rate $\left(\mathrm{k}_{\mathrm{off}}\right)$. As we had found no difference in the rate of CaM dissociation, we also looked at the relative rates of $\mathrm{CaM}$ association with wildtype and $\mathrm{C} \Delta 27 \mathrm{eNOS}$ (Fig. 4.14). Rates of CaM association were indistinguishable for these enzymes, although the rapidity of binding precluded the determination of $\mathrm{K}_{\text {off }}$. Nonetheless, we conclude that truncation of ACE-2 does not significantly affect the affinity of eNOS for CaM binding and must occur by a different mechanism. This phenomenon will be discussed in more detail in part II. 


\subsubsection{Does it effect the $\mathrm{EC}_{50}$ for $\mathrm{Ca}^{2+}$ if the CaM-eNOS complexes are pre-formed}

The experiments described in the previous section only looked at the formation of eNOS complexes under conditions in which $\mathrm{Ca}^{2+}$ was limited. Under physiological conditions CaM-binding to eNOS, and hence activation, would occur during a spike in intracellular $\mathrm{Ca}^{2+}$. CaM-eNOS complexes would then dissociate, and hence inactivate eNOS, as intracellular $\mathrm{Ca}^{2+}$ returned to resting levels. We therefore investigated potential differences (hysterisis) in the $\mathrm{Ca}^{2+}$-dependence curve for inactivation vs. activation of eNOS proteins. Activity of wildtype and C $\Delta 27$ eNOS were assessed under each of two conditions. Firstly, CaM-eNOS complexes were allowed to pre-form at maximal levels of $\mathrm{Ca}^{2+}(\approx 1 \mu \mathrm{M})$ prior to addition of $\mathrm{Ca}^{2+}$ chelating buffers. Thus, this experimental paradigm reflected the achievement of a new equilibrium condition for bound $\mathrm{CaM}$ after dissociating from eNOS due to $\mathrm{Ca}^{2+}$ chelation (unbinding). Secondly, eNOS (in $\approx 1 \mu \mathrm{M} \mathrm{Ca}^{2+}$ ) was added to $\mathrm{Ca}^{2+}$-buffered solutions already containing CaM. In this setting, the levels of $\mathrm{Ca}^{2+}$ limited the equilibrium of association of CaM-eNOS complexes (binding). For both $\mathrm{C} \Delta 27$ and wildtype eNOS, the two different treatments did not demonstrate any significant difference in the level of free $\mathrm{Ca}^{2+}$ required for half maximal activity (Fig. 4.15, panels $\mathrm{A}$ and $\mathrm{B}$ ). Hence, the observed differences in $\mathrm{Ca}^{2+}$-dependence for activity for C $\triangle 27$ eNOS, compared to wildtype eNOS, could not be attributed to differences in the $\mathrm{Ca}^{2+}$-dependence for binding to these species. 

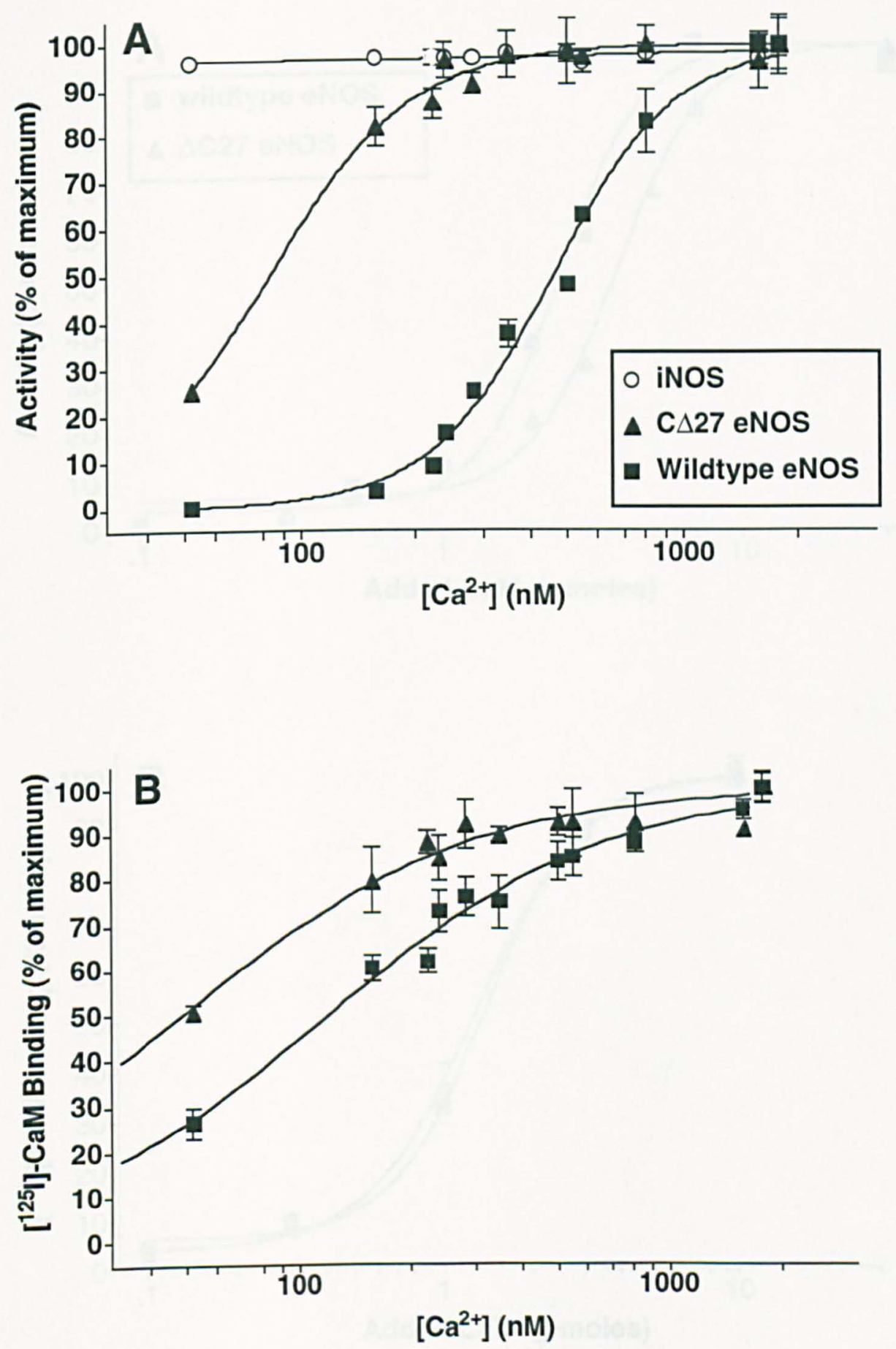

Fig. 4.11. Calcium-dependence of NO synthesis and calmodulin-binding for wild type and $C \triangle 27$ eNOS. Panel A: NO synthesis is shown as a percentage of the maximum rate observed in the presence of $1.8 \mu \mathrm{M} \mathrm{Ca}^{2+}$ for each eNOS protein (8 pmol). Panel B: Calmodulin binding is depicted as a percentage of complete occupancy, where $100 \%$ represents a 1:1 ratio of bound $\mathrm{CaM}$ to eNOS $(4 \mathrm{pmol})$. 

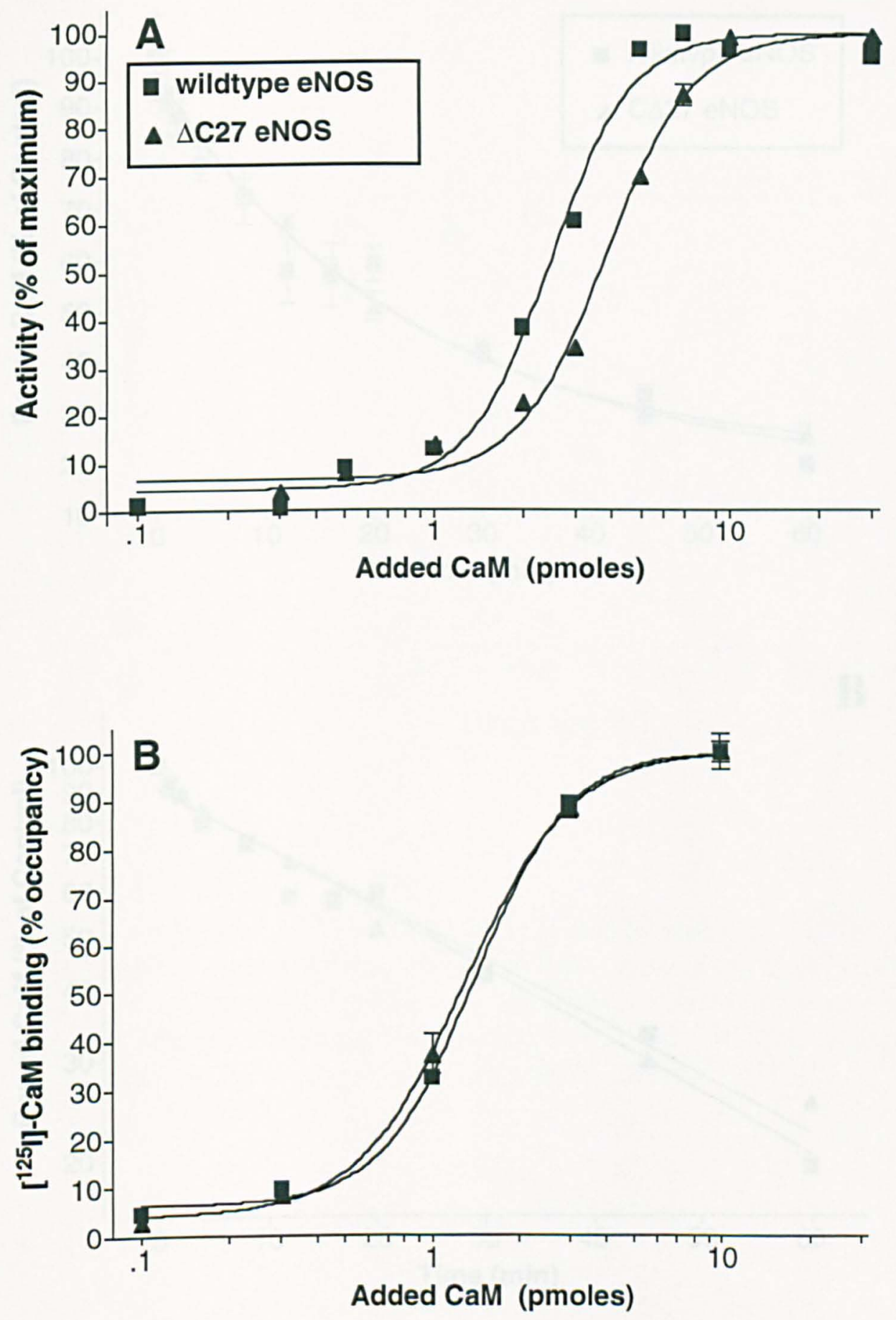

Fig. 4.12. Calmodulin affinity for wildtype and $\mathrm{C} \Delta 27$ eNOS. Panel A:Concentration dependence for CaM-induced NO synthesis by eNOS proteins $(4 \mathrm{pmol})$. Panel B:Concentration dependence for binding of $\left.{ }^{125} \mathrm{I}\right] \mathrm{CaM}$ to eNOS proteins $(2 \mathrm{pmol})$. Results are shown as percentage of maximum, as described for Fig. 4.11. 

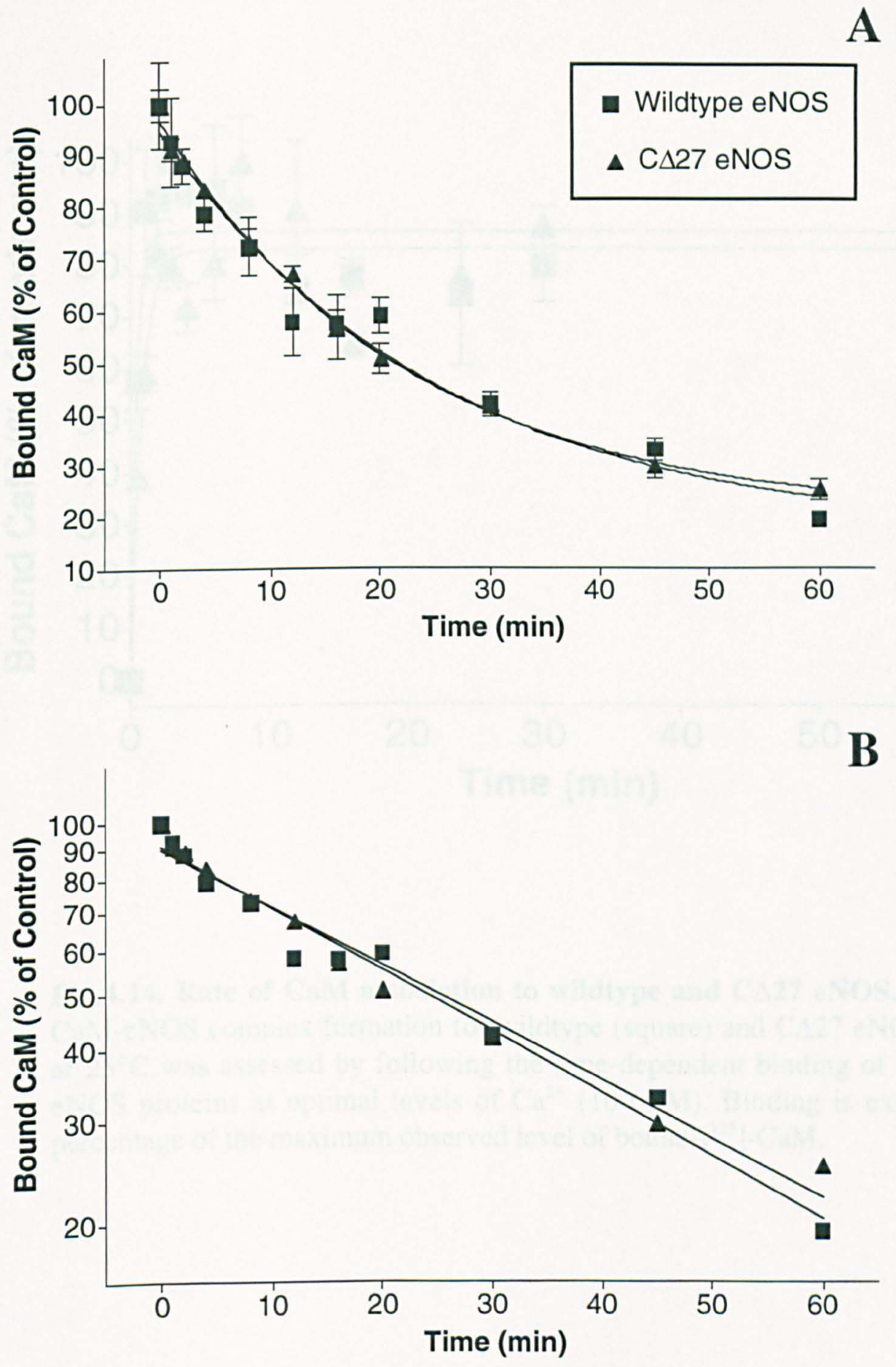

Fig. 4.13. Rate of $\left[{ }^{125} \mathrm{I}\right]-\mathrm{CaM}$ dissociation from wildtype and $\mathrm{C} \Delta 27$ eNOS. Panel $\mathrm{A}$ : the rate of $\mathrm{CaM}$ dissociation at $25^{\circ} \mathrm{C}$ from $\mathrm{CaM}$-eNOS complexes at maximal levels of $\mathrm{Ca}^{2+}$ $(100 \mu \mathrm{M})$ was assessed by following the time-dependent loss of bound $\left[{ }^{125} \mathrm{I}\right]$-CaM after the addition of a 3000-fold molar excess of unlabeled CaM. CaM binding is expressed as a percentage of the maximum observed prior to the addition of unlabeled CaM. Panel B: A semi-log plot of the above data allows the derivation of a dissociation rate by linear regression. 


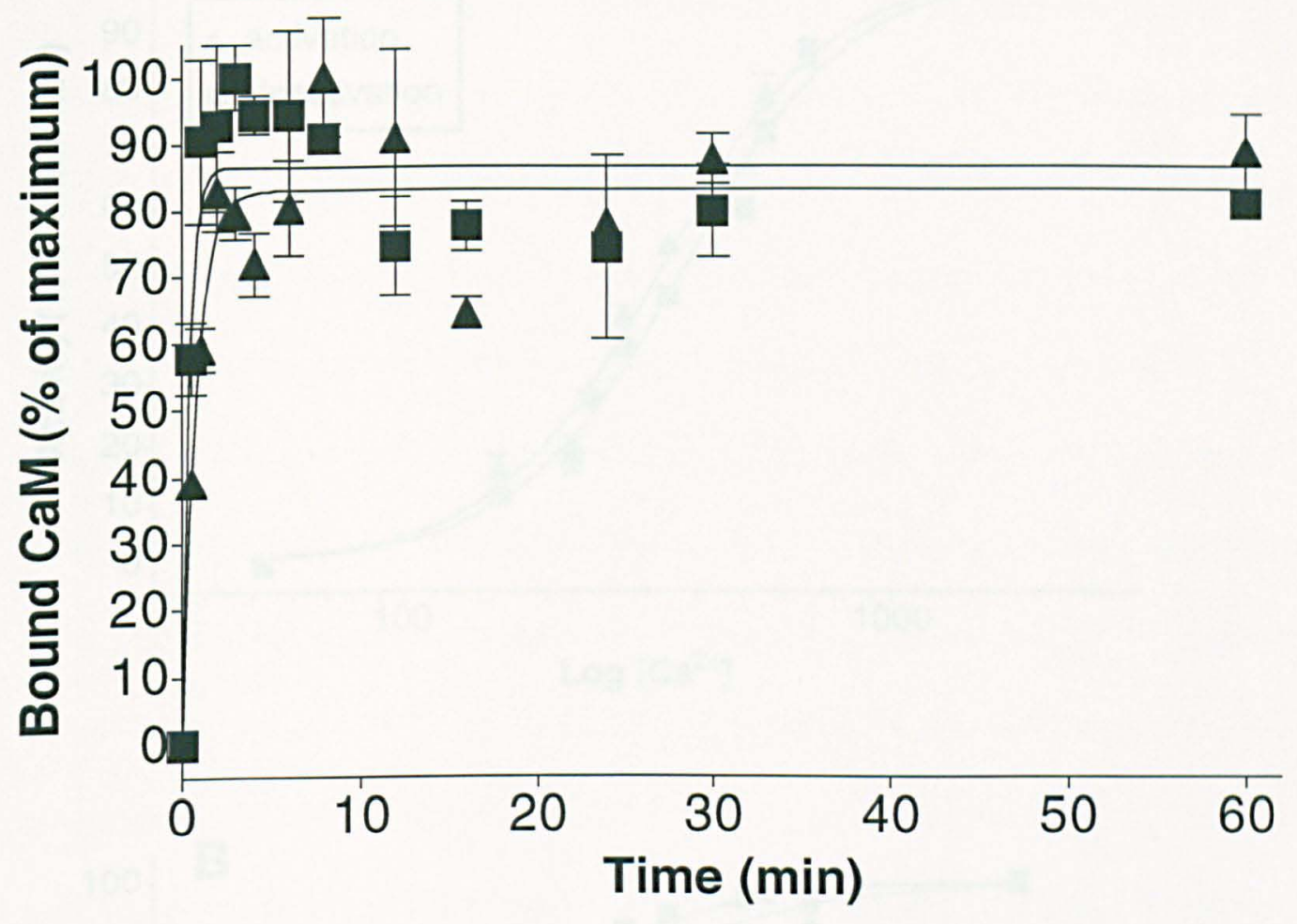

Fig.4.14. Rate of CaM association to wildtype and $\mathrm{C} \Delta 27 \mathrm{eNOS}$. The rate of CaM-eNOS complex formation for wildtype (square) and C $\Delta 27$ eNOS (triangle) at $25^{\circ} \mathrm{C}$ was assessed by following the time-dependent binding of $\left[{ }^{125}\right]-\mathrm{CaM}$ to eNOS proteins at optimal levels of $\mathrm{Ca}^{2+}(100 \mu \mathrm{M})$. Binding is expressed as a percentage of the maximum observed level of bound- $\left[{ }^{125}\right]-\mathrm{CaM}$. 

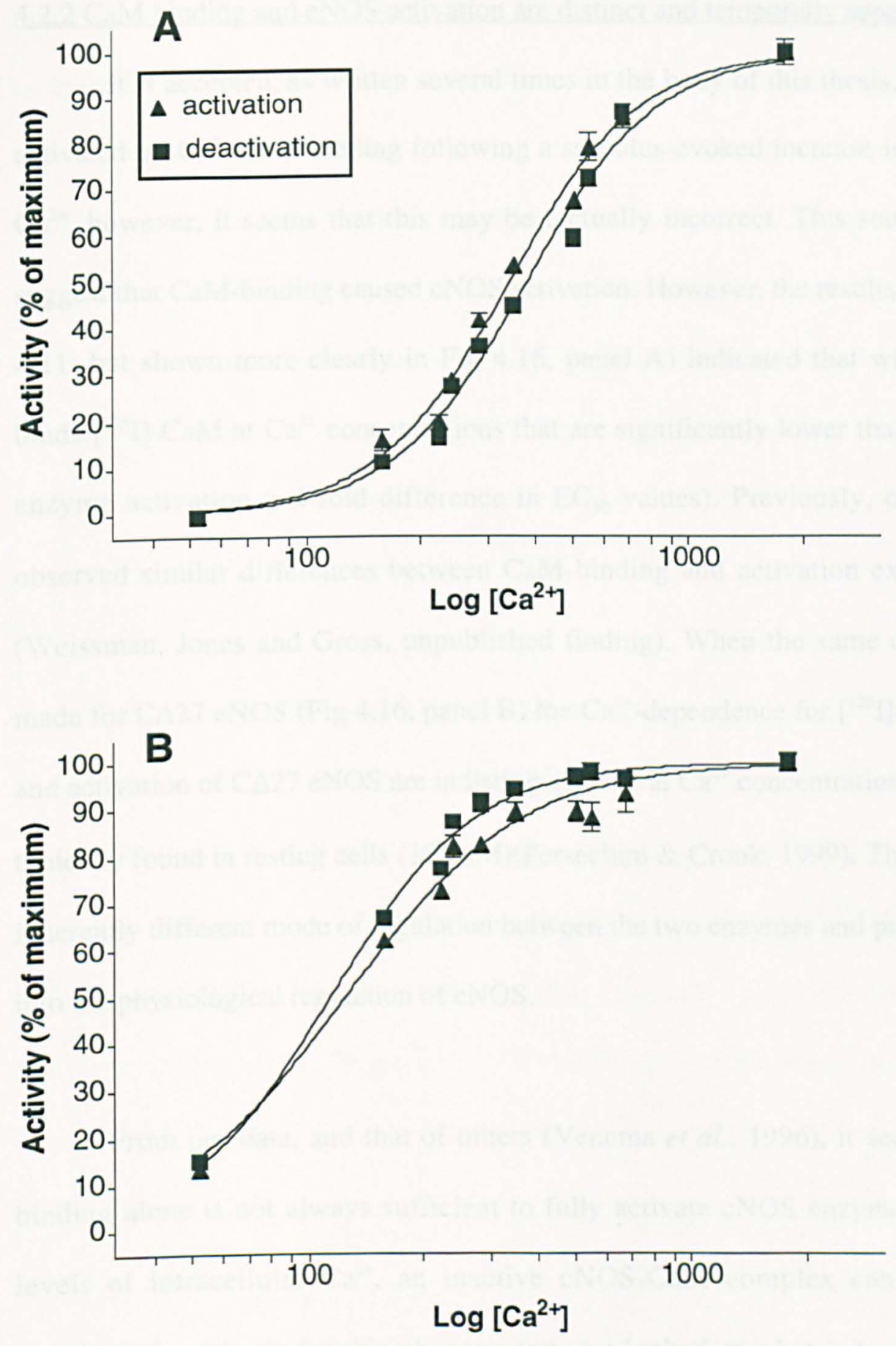

Fig. 4.15. $\mathrm{Ca}^{2+}$-dependence of activation vs. deactivation for wildtype and C $\triangle 27$ eNOS. $\mathrm{Ca}^{2+}$-dependence of eNOS activity was analyzed under conditions where CaM-eNOS complexes were allowed to form at defined levels of $\mathrm{Ca}^{2+}$ (activation) or to pre-form at $\approx 1 \mu \mathrm{M} \mathrm{Ca}^{2+}$ prior to transfer to the $\mathrm{Ca}^{2+}$ solutions (deactivation). Assay mixtures contained $100 \mathrm{nM} \mathrm{CaM}$ and 8 pmoles of wildtype (Panel A) or C $\Delta 27$ eNOS (Panel B).Values represent the mean of triplicate experiments \pm SEM. 
It is accepted, as written several times in the body of this thesis, that cNOS is activated by $\mathrm{Ca}^{2+} / \mathrm{CaM}$ binding following a stimulus-evoked increase in intracellular $\mathrm{Ca}^{2+}$, however, it seems that this may be factually incorrect. This statement would suggest that CaM-binding caused cNOS activation. However, the results in part I (Fig. 4.11, but shown more clearly in Fig 4.16, panel A) indicated that wildtype eNOS binds $\left[{ }^{125} \mathrm{I}\right]-\mathrm{CaM}$ at $\mathrm{Ca}^{2+}$ concentrations that are significantly lower than required for enzyme activation ( $\approx 4$-fold difference in $\mathrm{EC}_{50}$ values). Previously, our laboratory observed similar differences between CaM-binding and activation exist for nNOS (Weissman, Jones and Gross, unpublished finding). When the same comparison is made for $\mathrm{C} \Delta 27$ eNOS (Fig 4.16, panel B) the $\mathrm{Ca}^{2+}$-dependence for [ $\left.{ }^{125} \mathrm{I}\right]-\mathrm{CaM}$ binding and activation of $\mathrm{C} \Delta 27 \mathrm{eNOS}$ are indistinguishable at $\mathrm{Ca}^{2+}$ concentrations above those typically found in resting cells $(100 \mathrm{nM})($ Persechini \& Cronk, 1999). This suggests an inherently different mode of regulation between the two enzymes and provides insight into the physiological regulation of eNOS.

From our data, and that of others (Venema et al., 1996), it seems that CaM binding alone is not always sufficient to fully activate cNOS enzymes and, at low levels of intracellular $\mathrm{Ca}^{2+}$, an inactive cNOS-CaM complex can exist. When searching for a basis for this phenomenon, we looked at what is known about the regulation of cNOSs, and other CaM-dependent enzymes by $\mathrm{Ca}^{2+} / \mathrm{CaM}$.

From the study of $\mathrm{CaM}$ itself, we gained an appreciation that $\mathrm{CaM}$ can interact with target proteins via one or both of its two calcium-binding lobes (see Fig. 4.26). $\mathrm{CaM}$ is a $17 \mathrm{KD}$ protein composed of an 8-turn central $\alpha$-helix, flanked by globular 
$\mathrm{N}$-terminal and C-terminal $\mathrm{Ca}^{2+}$-binding lobes (Babu et al., 1988). Each lobe consists of two EF-hand motifs that possess the capacity for binding a single $\mathrm{Ca}^{2+}$ atom (Persechini et al., 1989). Binding of $\mathrm{Ca}^{2+}$ causes a conformational change, exposing hydrophobic and acidic residues which engage in binding to the relevant regulatory sites on target proteins (Weinstein \& Mehler, 1994). It has been shown that the Cterminal lobe of $\mathrm{CaM}$ binds $\mathrm{Ca}^{2+}$ with a greater affinity (approximately 10 -fold) than the N-terminal lobe (Linse et al., 1991). Hence, at basal levels of $\mathrm{Ca}^{2+}$, one lobe of $\mathrm{CaM}$ will exist partially in the active binding conformation, while the other remains in an inactive non-binding conformation.

Binding by cNOSs of a $\mathrm{CaM}$ species that is only partially $\mathrm{Ca}^{2+}$-saturated is supported by the fact that each lobe of $\mathrm{CaM}$ can bind to nNOS independently (Persechini et al., 1994) and that binding of the N-terminal lobe mediates nNOS activation (Persechini et al., 1996). Given this large disparity in $\mathrm{Ca}^{2+}$ affinity between the two lobes of CaM, it is not surprising that $\left[{ }^{125} \mathrm{I}\right]-\mathrm{CaM}$-binding studies indicate that the C-terminal lobe of CaM can be bound to both nNOS (Weissman, Jones and Gross, unpublished observation) and eNOS at resting concentrations of intracellular $\mathrm{Ca}^{2+}$ (100nM).

We therefore utilized wildtype $\mathrm{CaM}$, in conjunction with $\mathrm{CaM}$ constructs which were engineered to express two C-terminal (CC-CaM) or two N-terminal (NNCaM) lobes (Persechini et al., 1994), to investigate the activation of eNOS and C $\Delta 27$ eNOS by $\mathrm{Ca}^{2+} / \mathrm{CaM}$. 


\subsubsection{Activation of wildtype and $\mathrm{C} \triangle 27 \mathrm{eNOS}$ by engineered CaM proteins}

Before we assessed the effects of engineered CaM proteins on enzyme activation, it was first necessary to establish that these proteins were capable of complex formation with wildtype and $\mathrm{C} \Delta 27$ eNOS. As ${ }^{125} \mathrm{I}$-labeled CC-CaM and NN$\mathrm{CaM}$ were unavailable, we compared the ability of these engineered proteins to competitively inhibit the binding of $\left[{ }^{125} \mathrm{I}\right]-\mathrm{CaM}$ to eNOS proteins. As shown in Fig. 4.17 (panel A), at a concentration of $50 \mathrm{nM}$ wildtype (NC-CaM), NN-CaM and CC$\mathrm{CaM}$ all potently inhibited the binding of $\left[{ }^{125} \mathrm{I}\right]-\mathrm{CaM}(1 \mathrm{nM})$ to wildtype eNOS (94.7 $\pm 0.5,92.8 \pm 0.3$ and $86.5 \pm 1.5 \%$ inhibition, respectively). This indicates that all engineered CaMs had the ability to form complexes with wildtype eNOS. A similar pattern of competitive inhibition was observed for the various $\mathrm{CaM}$ proteins with C $\triangle 27$ eNOS (Fig. 4.17, panel B), although with slightly less potency $(93.4 \pm 2.0$, 85.6 \pm 2.0 and $78.0 \pm 1.5 \%$ inhibition, by $\mathrm{CN}-, \mathrm{NN}$ - and $\mathrm{CC}-\mathrm{CaM}$, respectively). Given that binding of eNOS by these engineered CaM proteins was confirmed, we investigated their ability to elicit activation of wildtype and C $\triangle 27$ eNOS.

Like NC-CaM, NN-CaM elicited full activation of eNOS, but CC-CaM failed to evoke any detectable NO synthesis (Fig. 4.18). This concurred with the previous findings of nNOS by Persechini et al, showing that although both lobes of CaM are capable of interactions with cNOSs, binding of the N-terminal lobe is essential for activation (Persechini et al., 1996). Predictably, C $\Delta 27$ eNOS was fully activated by NN-CaM, but interestingly was also partially activated by CC-CaM. CC-CaM elicited $29.8 \pm 4.3 \%$ of the activity of $C \Delta 27$ eNOS, relative to that observed in the presence of NC-CaM (Fig. 4.18). It is possible that higher concentrations of CC-CaM would elicit fuller activation of $\mathrm{C} \Delta 27$ eNOS, however, we were unable to test this due to limited 
CC-CaM availability (a one-time gift from Dr. Persechini). The fact that CC-CaM 1

was able to elicit partial activation of $\mathrm{C} \Delta 27$ eNOS, but not wildtype eNOS, suggests that there are distinct differences in the mode of CaM-dependent activation of the two enzymes.

To further investigate this phenomenon, we looked at the ability of $\mathrm{NN}$ - and CC-CaM to activate the reductase domain of wildtype and $\mathrm{C} \Delta 27$ eNOS. For wildtype eNOS (Fig. 4.19, panel A), NN-CaM, as expected, elicited full activation of cytochrome c reductase activity. Interestingly, $\mathrm{CC}-\mathrm{CaM}$ was able to elicit a modest increase in the rate of cytochrome c reduction $(29.6 \pm 7.8 \%$ of NC-CaM-dependent activity). C $\Delta 27$ eNOS (Fig. 4.19, panel B) also exhibited full activity in the presence of bound NN-CaM, and exhibited a similar level of increased activity in the presence of CC-CaM $(25.88 \pm 0.5 \%$ of NC-CaM-increased activity). When ferricyanide reduction was examined, $\mathrm{NN}-\mathrm{CaM}$ again elicited near full activation of wildtype eNOS ( $66.3 \pm 16.2 \%$ of NC-CaM-increased activity) (Fig. 4.20, panel A). However, CC-CaM did not elicit any increase in the rate of ferricyanide reduction. Conversely, C $\triangle 27$ eNOS-dependent ferricyanide reduction was fully activated by either NN-CaM or CC-CaM (145.0 $\$ 3.3 \%$ and $90.5 \pm 2.2 \%$ of NC-CaM-increased activity, respectively). This again suggests fundamental differences in CaM-dependent regulation between wildtype and $\mathrm{C} \triangle 27 \mathrm{eNOS}$.

From our experiments with CC-CaM, there are two reasonable explanations to consider for the activation of $\mathrm{C} \Delta 27$ eNOS in the absence of an N-terminal lobe of $\mathrm{CaM}$, an apparent prerequisite for activation of wildtype eNOS. One possibility is that binding of a single $\mathrm{C}$-lobe of $\mathrm{CaM}$ is sufficient to elicit the partial activation of $\mathrm{C} \Delta 27$, 
but not wildtype eNOS. The second possible scenario is that both lobes of CaM are required for activation of $\mathrm{C} \Delta 27$ eNOS, but one of the C-lobes of CC-CaM can substitute for the normally present $\mathrm{N}$-lobe. The latter explanation would require that truncation of ACE-2 modifies the critical interactions between eNOS and the N-lobe of $\mathrm{CaM}$ that lead to enzyme activation. We hypothesize that the presence of ACE-2, directly or indirectly, acts as an obstruction to binding for the N-lobe of CaM.

To test this, we compared the $\mathrm{Ca}^{2+}$-dependence curves for activation of wildtype and $\mathrm{C} \Delta 27$ eNOS by NN-CaM. As expected, due to the low-affinity $\mathrm{Ca}^{2+}-$ binding exhibited by the N-lobe of CaM (compared with the C-lobe), $\mathrm{Ca}^{2+}$ dependence curves for both enzymes were significantly shifted to the right, vs. NC$\mathrm{CaM}$ (Fig. 4.21). However, the $\mathrm{EC}_{50}$ of $\mathrm{Ca}^{2+}$ for activation of $\mathrm{C} \Delta 27$ eNOS was significantly different to that for wildtype eNOS $\left(\mathrm{EC}_{50} 853.1 \pm 3.10\right.$ and $\left.1089.0 \pm 10.4\right)$. This result supports our hypothesis that the presence of ACE-2 primarily affects the interactions of eNOS with the N-lobe of $\mathrm{CaM} . \mathrm{Ca}^{2+}$-dependence curves for $\left[{ }^{125} \mathrm{I}\right]-\mathrm{CaM}$ binding, dependent on interactions between eNOS and the C-lobe of CaM, show very little difference between wildtype and $\mathrm{C} \Delta 27$ eNOS. However, $\mathrm{Ca}^{2+}$-dependence curves for CaM-dependent activation, largely dependent on interactions between eNOS and the N-lobe of CaM, demonstrate a 5-fold reduction in $\mathrm{EC}_{50}$ of $\mathrm{Ca}^{2+}$ of CA27 eNOS. The above experiment, using NN-CaM demonstrates that the interactions between the N-lobe of CaM and eNOS are indeed different between wild type and $C \triangle 27$ eNOS. 

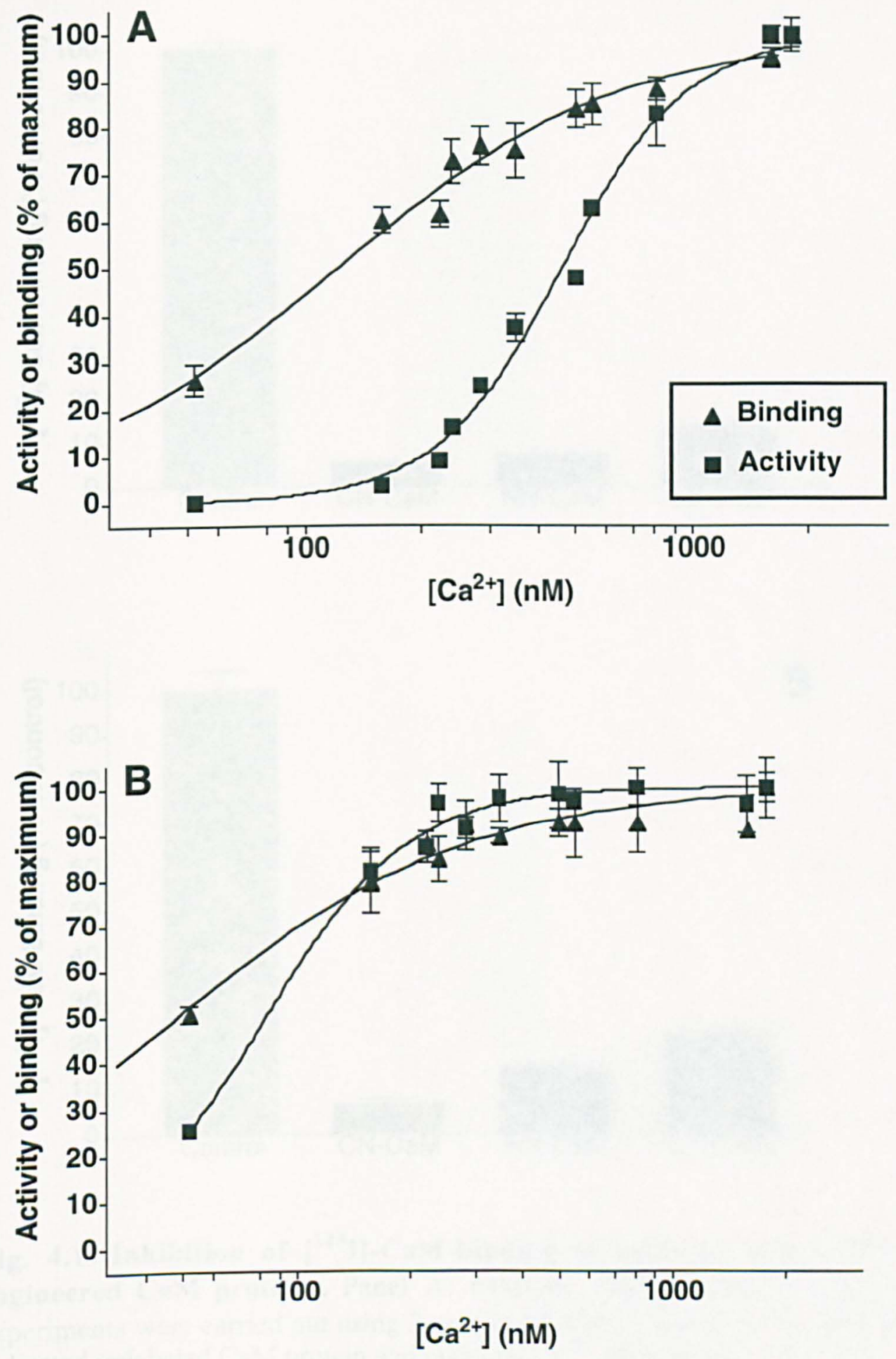

Fig. 4.16. Comparison of $\mathrm{Ca}^{2+}$-dependencies for CaM-binding and activation of wildtype and $\mathbf{C} \Delta \mathbf{2 7}$ eNOS. Using the data depicted in Fig. 4.11, the relative disparity between $\mathrm{CaM}$ occupancy and enzyme activation for wildtype (Panel A) and CA27 eNOS (Panel B) is shown. 

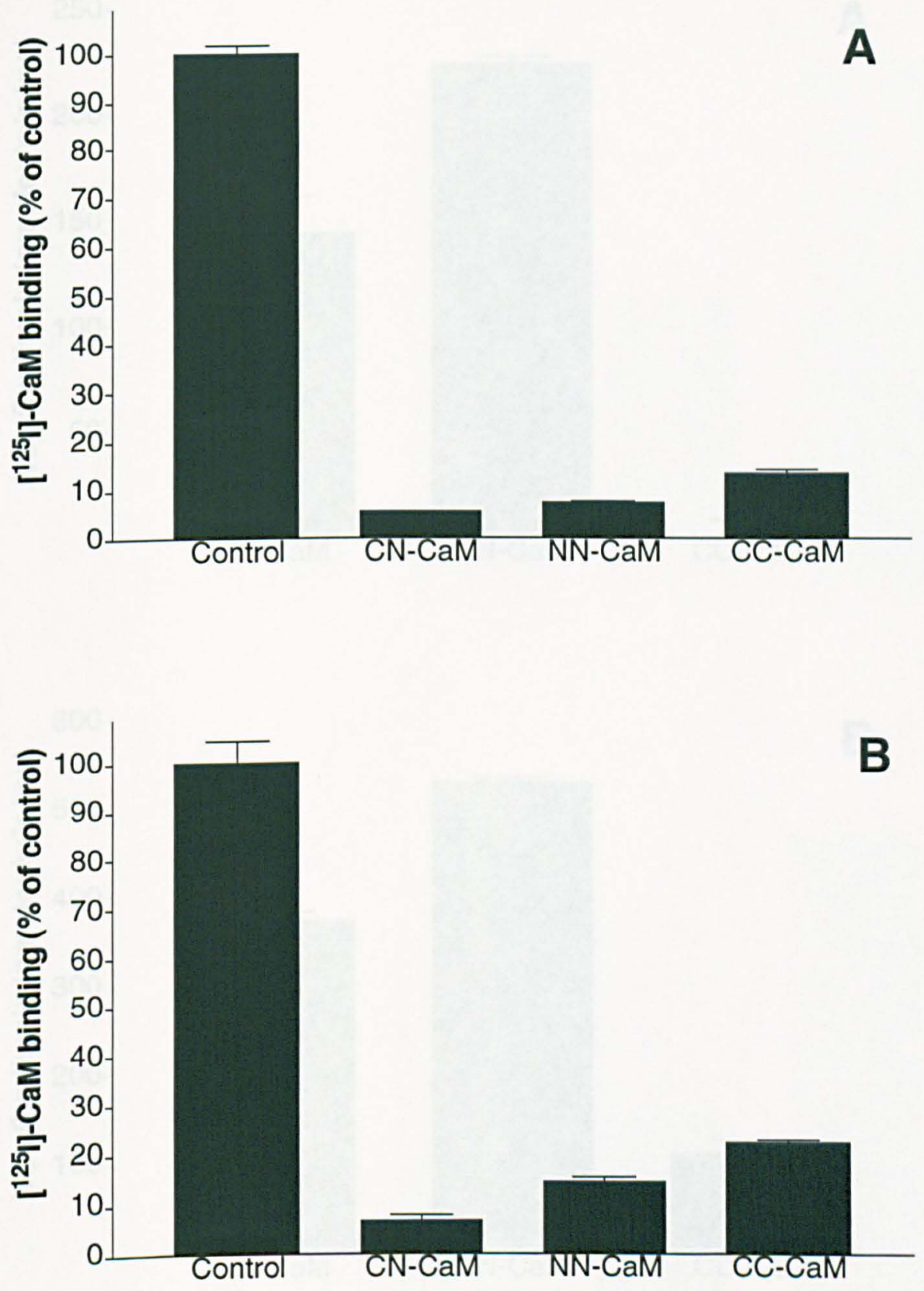

Fig. 4.17.Inhibition of $\left[{ }^{125} \mathrm{I}\right]-\mathrm{CaM}-b i n d i n g$ to wildtype and $\mathrm{C} \Delta 27$ eNOS by engineered CaM proteins. Panel A: wildtype eNOS. Panel B: C $\Delta 27$ eNOS. All experiments were carried out using 2 pmoles of NOS, $1 \mathrm{nM}$ of $\left[{ }^{125} \mathrm{I}\right]-\mathrm{CaM}, 50 \mathrm{nM}$ of the indicated unlabeled $\mathrm{CaM}$ protein and maximal $\mathrm{Ca}^{2+}$ concentrations $(100 \mu \mathrm{M})$. 

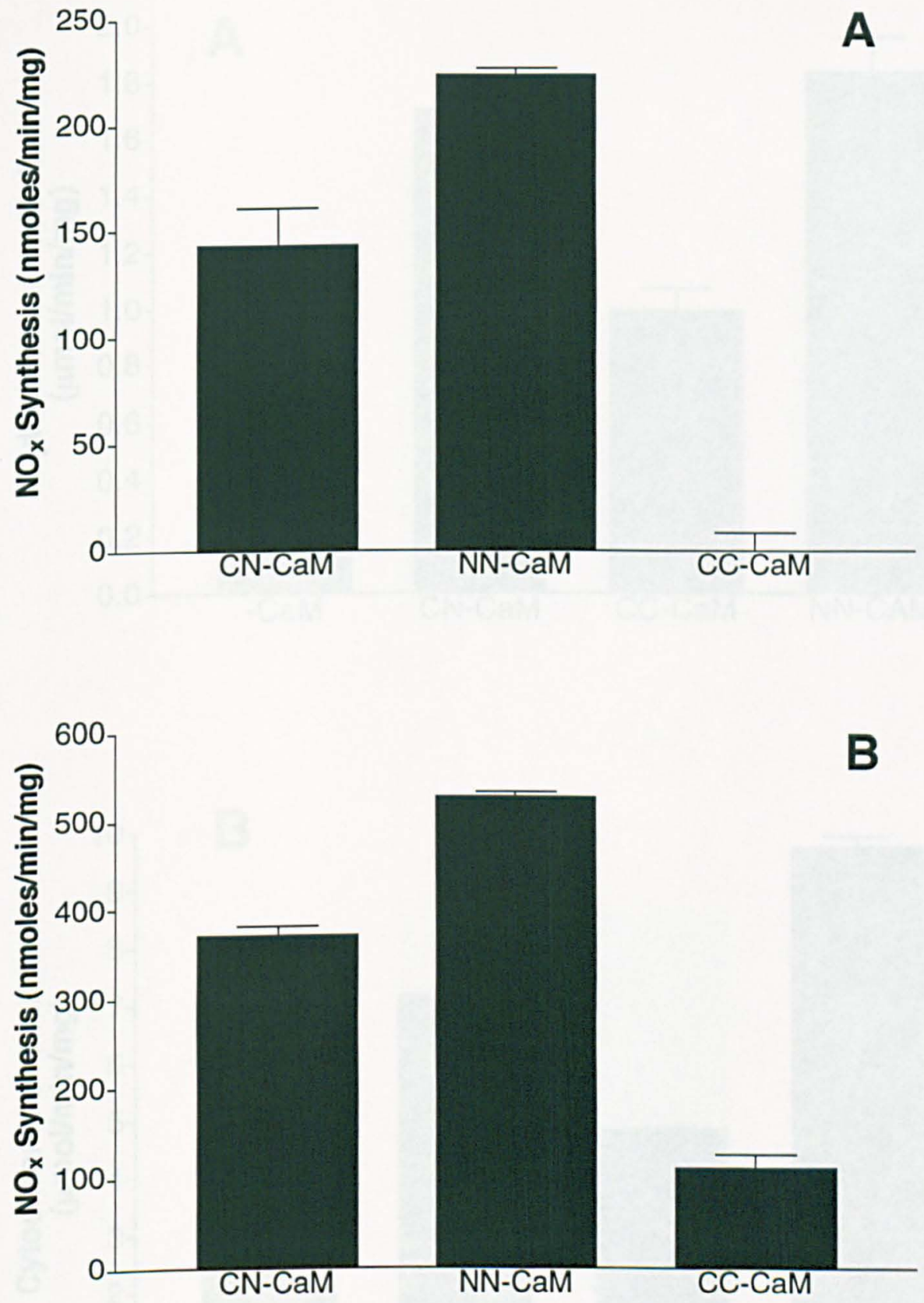

Fig. 4.18. Rates of NO synthesis by wildtype and C $\triangle 27$ eNOS in the presence of engineered CaM proteins. Panel A: wildtype eNOS Panel B: CA27 eNOS. All experiments were carried out using 8 pmoles of NOS, $100 \mathrm{nM}$ of the relevant CaM protein and optimal $\mathrm{Ca}^{2+}$ concentrations $(100 \mu \mathrm{M})$. CaM-free measurements of activity were obtained in the presence of $5 \mathrm{mM}$ EGTA and rates are derived from the mean of two separate preparations of each enzyme, assayed in triplicate. 

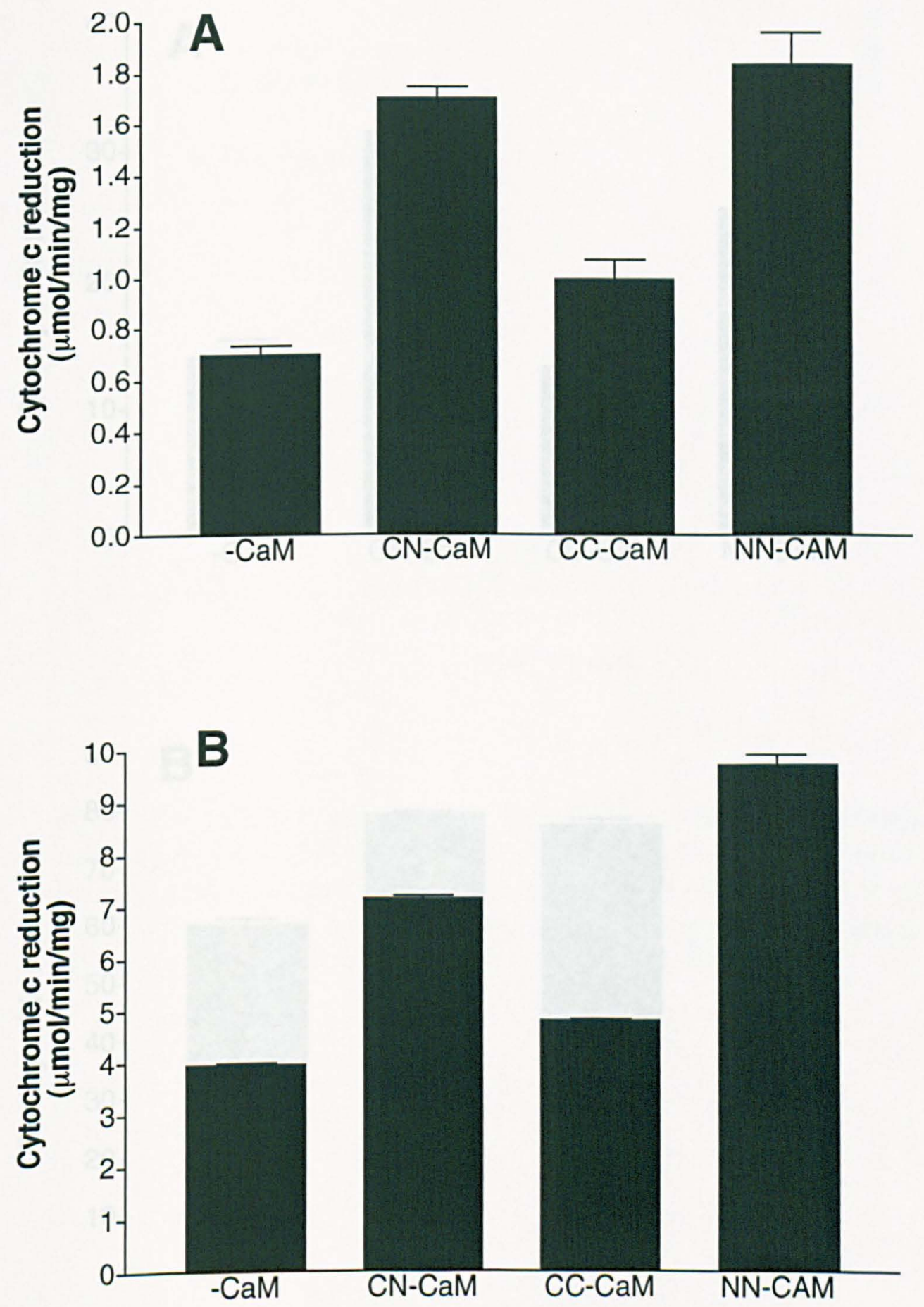

Fig. 4.19. Ability of engineered CaM proteins to elicit activation of cytochrome $\mathrm{c}$ reduction by wildtype and $\mathrm{C} \Delta 27$ eNOS. Panel A: wildtype eNOS. Panel B: C $\Delta 27$ eNOS. All reaction $\mathrm{s}$ were carried out using 1 pmole of NOS, $100 \mu \mathrm{M} \mathrm{Ca}^{2+}$ and $100 \mathrm{nM}$ of the indicated $\mathrm{CaM}$ protein. Values are the mean of triplicate experiments \pm SEM. 

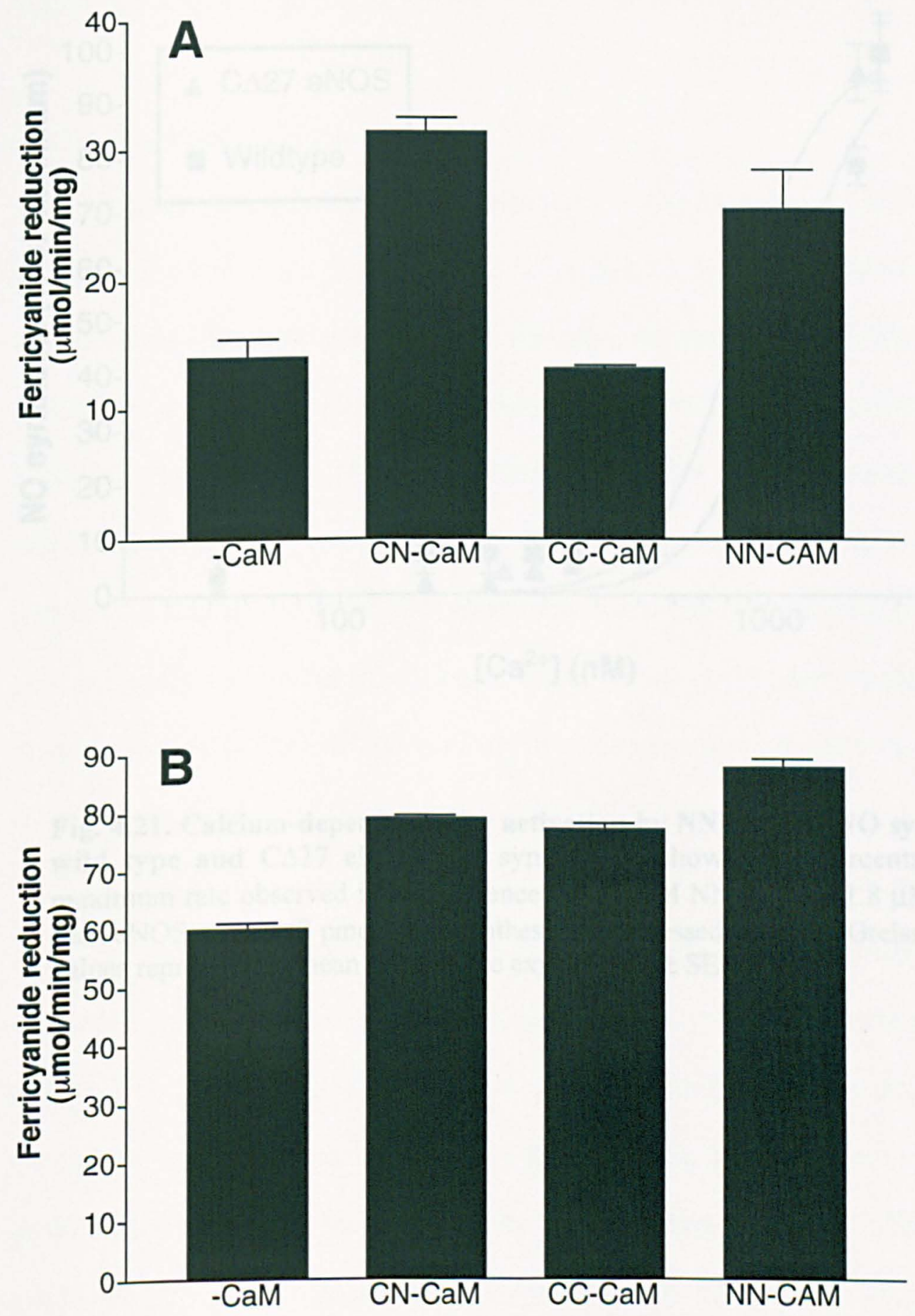

Fig.4.20. Ability of engineered CaM proteins to elicit activation of ferricyanide reduction by wildtype and $\mathrm{C} \Delta 27$ eNOS. Panel A: wildtype eNOS. Panel B: $\mathrm{C} \Delta 27$ eNOS. All reaction s were carried out using 0.1 pmoles of NOS, $100 \mu \mathrm{M} \mathrm{Ca}^{2+}$ and 10 $\mathrm{nM}$ of the indicated $\mathrm{CaM}$ protein. Values represent the mean of triplicate experiments \pm SEM. 


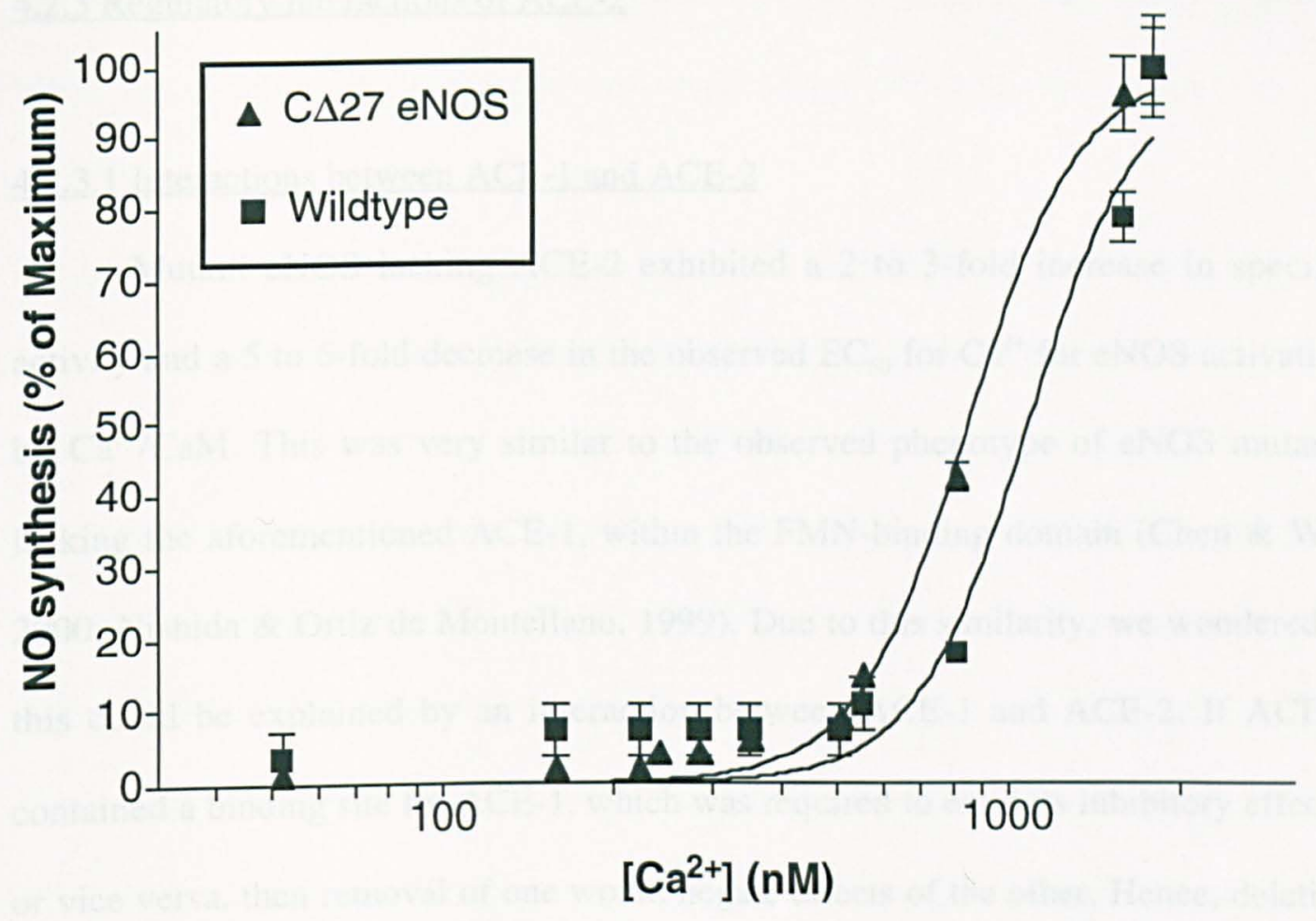

Fig. 4.21. Calcium-dependence for activation by NN-CaM of NO synthesis by wild type and $\mathrm{C} \triangle 27$ eNOS. NO synthesis is shown as a percentage of the maximum rate observed in the presence of $100 \mathrm{nM} \mathrm{NN}-\mathrm{CaM}$ at $1.8 \mu \mathrm{M} \mathrm{Ca}^{2+}$ for each eNOS protein ( $8 \mathrm{pmol})$. NO synthesis was assessed using the Greiss assay and values represent the mean of triplicate experiments \pm SEM. 


\subsubsection{Regulatory interactions of ACE-2}

\subsubsection{Interactions between $A C E-1$ and ACE-2}

Mutant eNOS lacking ACE-2 exhibited a 2 to 3 -fold increase in specific activity and a 5 to 6 -fold decrease in the observed $\mathrm{EC}_{50}$ for $\mathrm{Ca}^{2+}$ for eNOS activation by $\mathrm{Ca}^{2+} / \mathrm{CaM}$. This was very similar to the observed phenotype of eNOS mutants lacking the aforementioned ACE-1, within the FMN-binding domain (Chen \& Wu, 2000; Nishida \& Ortiz de Montellano, 1999). Due to this similarity, we wondered if this could be explained by an interaction between ACE-1 and ACE-2. If ACE-2 contained a binding site for ACE-1, which was required to exert its inhibitory effects, or vice versa, then removal of one would negate effects of the other. Hence, deletion of ACE-1 or ACE-2 could have similar effects because they are affecting the same inhibitory machinery.

To test this theory we utilized the ACE-1-derived peptides described in chapter 3 , specifically eNOS $_{617-639}$. This peptide forms an inhibitory complex with wildtype eNOS, displacing bound $\mathrm{CaM}$ and preventing enzyme activation. The location of the ACE-1 binding site on eNOS is unknown; we hypothesized that if this peptide exerts its effects by an interaction with a binding site within ACE-2, C $\triangle 27$ eNOS would be less susceptible to inhibition by the peptide. We compared the potency of eNOS ${ }_{617-639}$ to inhibit both activity of, and [ $\left.{ }^{125} \mathrm{I}\right]-\mathrm{CaM}$ binding to, wildtype and $C \Delta 27$ eNOS. Consistent with our hypothesis, $C \Delta 27$ eNOS was significantly less susceptible to inhibition of both activity $\left(\mathrm{IC}_{50}>100 \mu \mathrm{M}\right)$ and $\left[{ }^{125} \mathrm{I}\right]-\mathrm{CaM}$ binding $\left(\mathrm{IC}_{50}\right.$ $\approx 50 \mu \mathrm{M})$, compared to wildtype eNOS $\left(\mathrm{IC}_{50} \approx 30 \mu \mathrm{M}\right.$ and $\approx 15 \mu \mathrm{M}$, for activity and [25] $\mathrm{I}-\mathrm{CaM}$ binding, respectively) (Fig 4.22, panel A and B). Although these results 
are supportive of a regulatory interaction between ACE-1 and ACE-2, further biochemical and/or structural evidence is required to confirm this interaction.

\subsubsection{Interaction between $\mathrm{ACE}-2$ and heat shock protein 90}

The phenotype of ACE-2 truncated eNOS is also similar to that exhibited by eNOS when in a functional complex with heat shock protein 90 (HSP90). We wondered whether the binding of HSP90 involves interactions with ACE-2, that may disable the tonic inhibition of eNOS imposed by this region. Thus, we compared the ability of HSP90 to evoke increases in activity of wildtype and CA27 eNOS (Fig. 4.23). HSP90 caused a similar dose-dependent increase in the activity of both wildtype and CA27 eNOS. However, the maximum increase in either case was only $30-50 \%$ above control levels, a much smaller increase than the reported 2 to 3 -fold increase in activity (Garcia-Cardena et al., 1998). These results fail to support the view that eNOS activation by HSP90 complex formation does not involves ACE-2. 

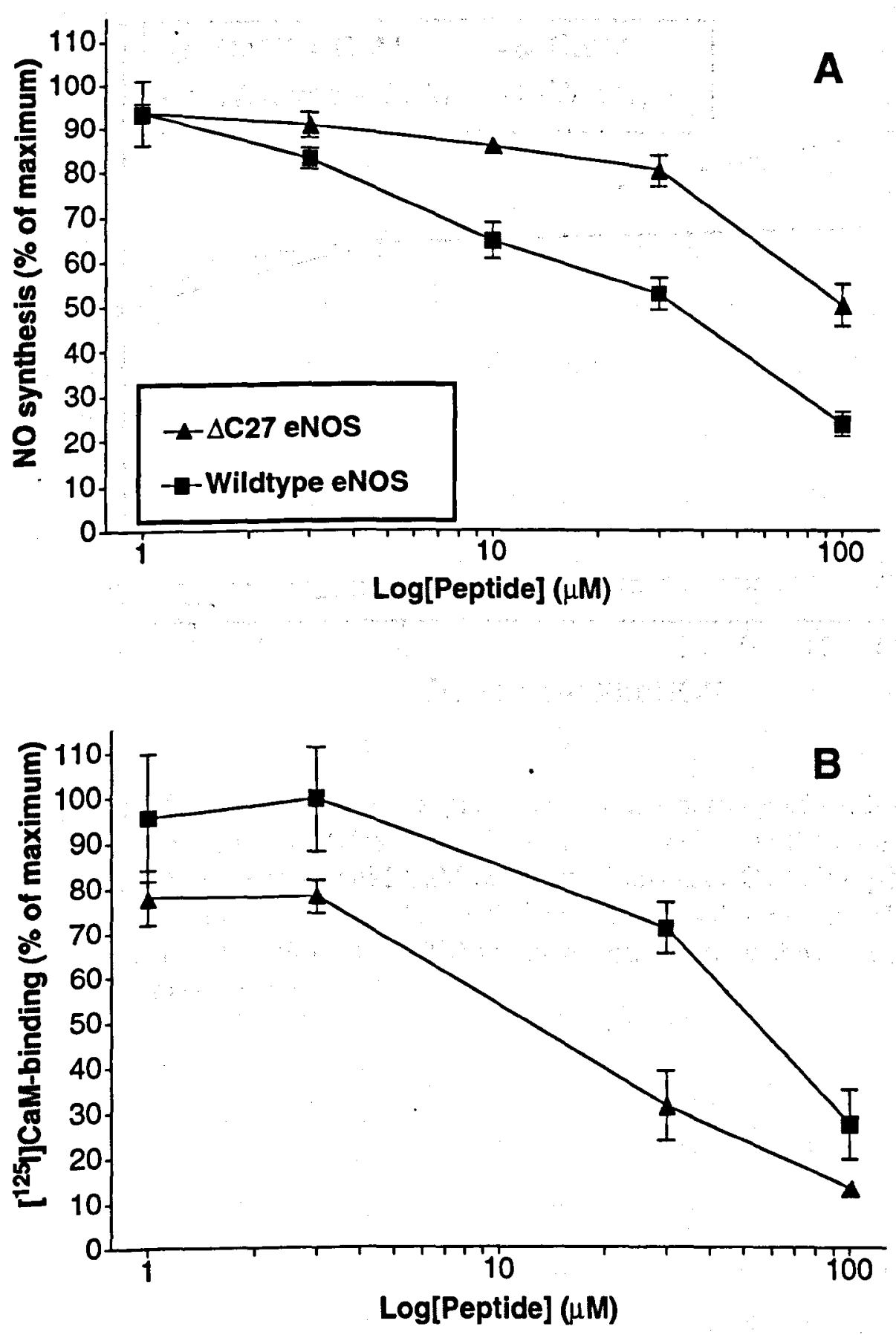

Fig. 4.22. Inhibition of wildtype and $\mathrm{C} \Delta 27 \mathrm{eNOS}$ by $\mathrm{eNOS}_{617-639}$, a peptide derived from the FMN autoinhibitory control element (ACE 1). Assays were performed using 10 pmoles of NOS per well and $100 \mathrm{nM} \mathrm{CaM}$ for both NO synthesis (Panel A) and $\left[{ }^{125} \mathrm{I}\right]-\mathrm{CaM}$-binding (Panel B). Values represent the mean of triplicate experiments \pm SEM. 


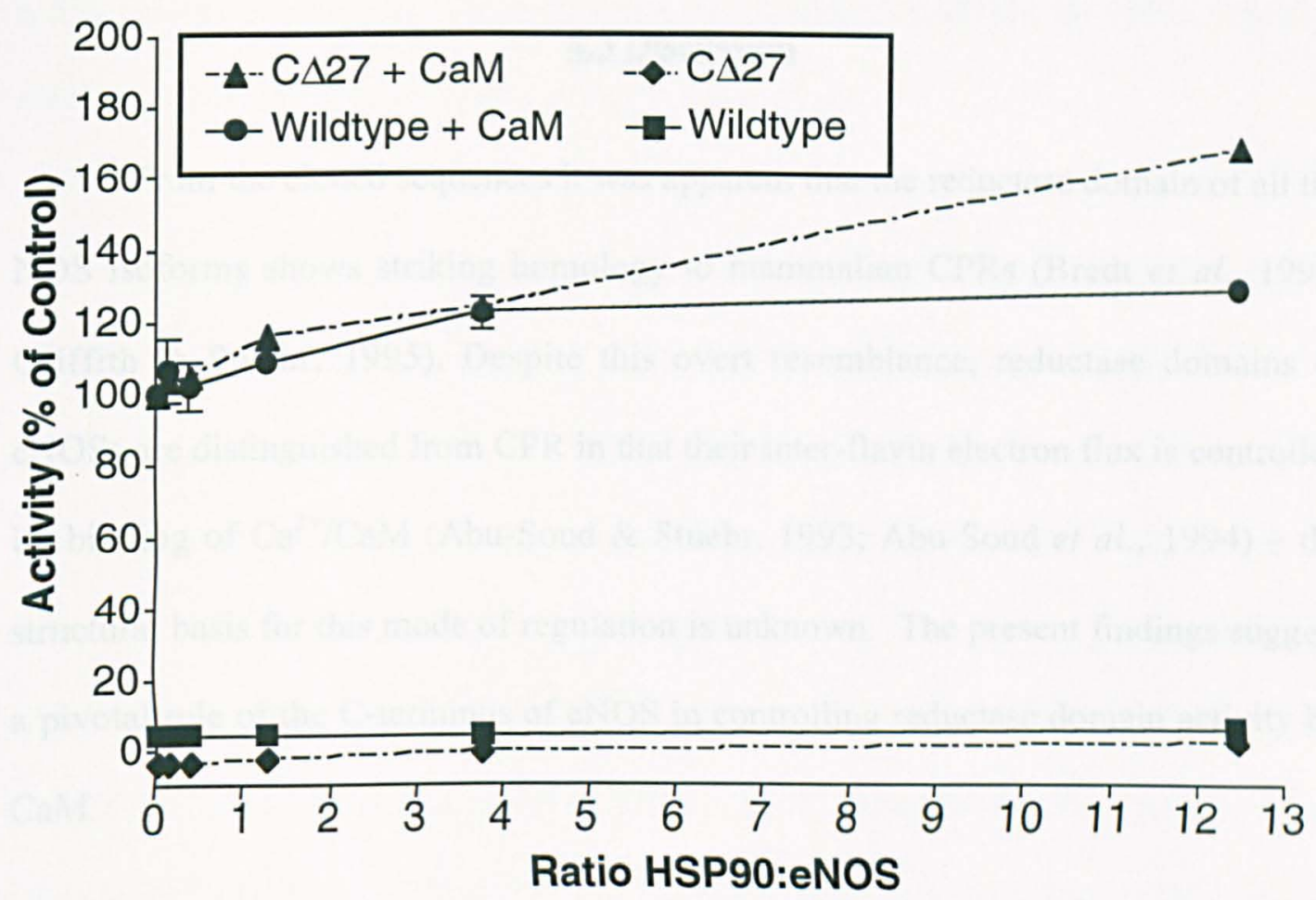

Fig. 4.23. Effect of heat shock protein 90 on the activity of wildtype and $\mathrm{C} \Delta 27$ eNOS. The activity of NOS (8 pmoles) was assessed using the Griess assay in the presence/absence of $100 \mathrm{nM} \mathrm{CaM}$ at maximal levels of $\mathrm{Ca}^{2+}(100 \mu \mathrm{M})$. The effect of added recombinant HSP90 on the activity of wildtype and C $\triangle 27$ eNOS is depicted as a function of HSP90 molar excess. Values shown are the mean of triplicate experiments \pm SEM. 


\subsection{Discussion}

From the cloned sequences it was apparent that the reductase domain of all the NOS isoforms shows striking homology to mammalian CPRs (Bredt et al., 1991; Griffith \& Stuehr, 1995). Despite this overt resemblance, reductase domains of cNOSs are distinguished from CPR in that their inter-flavin electron flux is controlled by binding of $\mathrm{Ca}^{2+} / \mathrm{CaM}$ (Abu-Soud \& Stuehr, 1993; Abu-Soud et al., 1994) - the structural basis for this mode of regulation is unknown. The present findings suggest a pivotal role of the C-terminus of eNOS in controlling reductase domain activity by CaM.

There are three segments of cNOSs for which CPR displays no homologue, the CaM binding peptide, the ACE peptide in the FMN subdomain, and a C-terminal peptide extension. The regulatory role of $\mathrm{CaM}$ and the ACE peptide are now well established, we therefore considered that the C-terminal extension might also serve a regulatory function in cNOSs. This view is in accord with recent demonstration that phosphorylation of a C-terminal Ser residue modulates eNOS activity in vivo (Chen et al., 1999; Dimmeler et al., 1999; Fulton et al., 1999).

Here we have shown that truncated eNOS, lacking 27 amino acids from the Cterminus, displays activity at dramatically reduced levels of free $\mathrm{Ca}^{2+}$ in comparison to wildtype eNOS. Truncation of the C-terminus also results in a 2-3 fold acceleration in maximum CaM-induced NO synthesis, associated with to an enhanced activity of the reductase domain, as measured by rates of cytochrome $\mathrm{c}$ and ferricyanide 
reduction. The truncation also imparts a diminished sensitivity to inhibition by a synthetic FMN domain ACE-derived peptide (ACE-1).

Independent investigations from the laboratory of Dr. Masters, simultaneously described a regulatory role for the C-termini of all three isoforms of NOS (Roman et al., 2000; Roman et al., 2000). For iNOS (Roman et al., 2000), mutants lacking all 21 amino acids of ACE-2, a 7 to 10-fold increase in reductase activities was described, however, NO synthesis was decreased $55-70 \%$. Using stopped-flow spectrophotometry, electron transfer into and between the two flavins, and from the flavins to the heme, was assessed to be 2 to 5 -fold faster in the absence of ACE- 2 . Similar mutants, lacking the entire ACE-2, were made for both of the cNOS isoforms with interesting results (Roman et al., 2000). Removal of the entire ACE-2 of rat nNOS (33 amino acids) or bovine eNOS (42 amino acids) resulted in an enzyme which exhibited CaM-free cytochrome c reductase activities that were 7 to 21 -fold higher than the corresponding wildtype cNOS. Unexpectedly, the addition of CaM resulted in a decrease in these rates, whereas CaM-bound wildtype and truncated NOSs reduced cytochrome $c$ at roughly similar rates. When the FAD-dependent reduction of ferricyanide was assessed, the results were slightly different. For nNOS, the truncated form exhibited CaM-free rates of ferricyanide reduction that were markedly higher than the wildtype, but unchanged by CaM-binding. Similarly, in the absence of CaM, the truncated form of eNOS exhibited a 2-fold greater rate of ferricyanide reduction than wildtype eNOS. However, while the addition of CaM caused an increase in activity of wildtype eNOS, ferricyanide reduction by the truncated forms was attenuated upon $\mathrm{CaM}$ binding. Thus, $\mathrm{CaM}$ enhanced the reductase functions of wildtype cNOSs, but acted as a partial, non-competitive 
inhibitor of the reductase functions of truncated mutants. When the ability of these proteins to synthesize NO was assessed, it was found that truncated forms of cNOS exhibit a significant increase in the rate of NO synthesis, compared to their cognate wildtype forms ( 45 and $33 \%$ increase for truncated nNOS and eNOS, respectively). Notably, the truncated proteins also exhibited CaM-independent synthesis of NO, albeit at low levels (14 and $20 \%$ of CaM-dependent NO synthesis for truncated nNOS and eNOS, respectively).

Combined with our own studies, the evidence from C-terminal truncation confirm the important regulatory function of the C-terminal extension (ACE-2) for all NOS isoforms (Roman et al., 2000; Roman et al., 2000). However, the mechanism by which ACE-2 exerts these inhibitory effects remains to be elucidated. Additionally, given that Akt/PKA-mediated phosphorylation of a Ser residue within ACE-2 of eNOS has been shown to be an in vivo form of regulation (Chen et al., 1999; Dimmeler et al., 2000; Dimmeler et al., 1999; Fulton et al., 1999; McCabe et al., 2000), our studies provide a possible mechanism for the regulation of eNOS by Akt/PKA.

\subsubsection{How does ACE-2 exert its autoinhibitory effects?}

As the reductase domain of NOSs exhibits a good degree of homology to cytochrome P450 reductase (CPR), we used the solved crystal structure of CPR (Wang et al., 1997) to gain insight into the possible regulatory functions of ACE-2. Obviously, as CPR lacks this region, this exercise provides no direct structural information about the C-terminus of eNOS. However, the relative positioning of the 
C-terminus in CPR is highly informative. Notably, the C-terminus of CPR curls back towards the main body of the enzyme and ends in a region proximal to the binding sites for both NADPH and FAD. As shown in Fig. 4.2, the penultimate residue of CPR, a Trp residue, lies at the interface between bound NADPH and FAD. The distance between bound NADPH and the isoalloxazine ring is at least $9 \mathrm{~A}^{\circ}$, a distance too great for efficient electron tunneling. This structure was derived using bound $\mathrm{NADP}^{+}$, and hence the distance may actually be reduced by a conformational shift when NADPH is bound to CPR, allowing efficient electron transfer.

In any event, the structure of CPR raise the possibility that the C-terminal residues play a role in electron transfer. The aromatic ring of the penultimate Trp of CPR is coplanar with the isoalloxazine ring of FAD and is positioned in a manner in which the $\pi$-cloud of electrons could reasonably modulate electron transfer between NADPH and FAD. It is possible that this $\pi$-cloud of electrons provides a route for electron flow, directly mediating the transfer of electrons from NADPH to FAD. It is equally possible that this $\pi$-cloud of electrons serves as an impediment to electron transfer, which must be displaced in order to facilitate electron transfer. As can be seen in Fig. 4.1, all NOSs contain a Phe residue, which is homologous to the Cterminal Trp of CPR. Given this arrangement, one can readily imagine that interactions of the C-terminus could cause subtle reorientation of this aromatic residue, and hence serve to regulate electron flux through the reductase domain. Importance of this Phe in NOS catalysis has already been demonstrated for iNOS; while activity is not diminished by removal of all subsequent $\mathrm{C}$-terminal amino acids, deletion of this Phe results in $71 \%$ loss in maximal activity (Xie et al., 1994). Conceivably, ACE-2 could extend to a point where it can modulate the transfer of 
electrons from FAD to FMN. The two flavin rings of FAD and FMN form contacts via their dimethyl benzene rings; it is possible that ACE- 2 could modulate both the angle and distance between these two rings. This could in turn regulate the flow of electrons between the two moieties. An interaction between ACE-2 and the reductase domain flavins is supported by evidence from truncation of the entire ACE-2. Firstly, removal of this region increases the rate of electron flow, both into (NADPH $\rightarrow$ FAD) and between (FAD $\rightarrow$ FMN) the flavins (Roman et al., 2000; Roman et al., 2000). Secondly, the presence of ACE-2 also stabilizes the one electron-reduced semiquinone form exhibited in intact iNOS, the semiquinone form could not be detected in truncated iNOS. Stabilization of the semiquinone may occur by ACE-2 limiting molecular oxygen's access to the "opening" of the FAD-FMN interface. This possibility may extend to artificial electron acceptors, cytochrome $\mathrm{c}$ and ferricyanide, providing an explanation for the enhanced reductase activities observed with $\mathrm{C}$ terminal-truncated NOSs.

Further consideration of CPR structure suggests an additional mechanism by which the C-terminal extension could serve to inhibit eNOS activity - obstruction of the trans-flow of electrons from the reductase domain of one monomer to the oxygenase domain of the other monomer. The structure of CPR is consistent with the possibility that C-terminal residues of NOS extend into the region of the dimer interface. If so, interactions with the FMN-domain autoinhibitory peptide may serve to modulate FMN-heme contacts required for NO synthesis. In one model, we envision that the C-terminal extension might impose a "wedge" that impedes electron transfer between the FMN of one monomer and the heme of its counterpart. A role for 
the ACE-2 in the control of inter-domain (FMN $\rightarrow$ heme) electron flow is supported by stopped-flow analyses of ACE-2 deleted NOSs. In truncated iNOS the rate limiting step for NO synthesis was no longer the supply of electrons from the reductase domain (heme reduction), as for wildtype iNOS, but was now limited by the rate of dissociation of the heme-nitrosyl complex. This would imply that ACE-2 interferes with the flow of electrons between reductase and oxygenase domains.

An intriguing possibility is that $\mathrm{ACE}-1$ and $\mathrm{ACE}-2$ function in concert, i.e. the autoinhibitory peptide in the FMN-binding domain confers inhibition by interacting with residues in the C-terminal peptide extension. If so, phosphorylation of Ser 1179 (or removal of the C-terminal extension) may activate eNOS by preventing this interaction. Several findings provide evidence consistent with an interaction between the C-terminus and the autoinhibitory domain. First, deletion of the autoinhibitory domain results in an enzyme with a decreased $\mathrm{Ca}^{2+}$-dependence for activation and a 2 3 fold increase in the rate of NO synthesis (Nishida \& Ortiz de Montellano, 1999), a near-identical phenotype to that observed for $\mathrm{C} \Delta 27$ eNOS. If the $\mathrm{C}$-terminus contains the binding site for the autoinhibitory domain, this would explain the similar phenotypes; inhibition can be equivalently relieved by removal of either autoinhibitory domain in toto, or by selective modification of key sites of interaction. Second, we found that truncation of the C-terminus of eNOS imparts a diminished sensitivity to inhibition by a synthetic autoinhibitory domain-derived peptide. The efficacy of such peptides for inhibiting CaM binding and activation of cNOSs was considered to result from binding to residues that dock with the endogenous autoinhibitory peptide (Salerno et al., 1997). The CPR crystal structure does not 
provide clear support for this interaction. Indeed, ACE-1 and ACE-2 would appear to originate from opposing sides of the reductase domain flavins, at too greater a distance to infer their interaction. However, given the lack of structural information for either ACE, the relative extension and positioning of the two is unknown and an interaction between the two remains possible.

Lastly, an interaction between $\mathrm{ACE}-2$ and bound $\mathrm{CaM}$ is a possibility; consideration of the relative positioning of the two may be telling. Since CaM-binds at the N-terminal side of the reductase domain, it would likely be situated on the reface of the FAD isoalloxazine ring, with ACE-2 covering the other side of the flavins. Therefore, CaM and ACE-2 could modulate electron flow by adjusting the relative distance and/or orientation of the two isoalloxazine rings and bound NADPH. This may additionally involve the alignment of FMN-heme contacts required for the synthesis of NO. By this model, the effects of CaM would be opposed to those of ACE-2; while CaM induces positive effects, aligning the moieties for efficient electron transfer, ACE-2 may exert negative effects that disrupt this alignment.

The system outlined above provides a basis to interpret the ACE-1/ACE-2derived peptide studies described in results section. Nonetheless, a conundrum exists, although both regions obviously serve an autoinhibitory function, ACE-derived peptides are potent inhibitors of eNOS activity (and CaM binding), while ACE-2derived peptides are weakly inhibitory only at very high concentrations $(>100 \mu \mathrm{M})$. This may be reconciled as a simple issue of peptide access. By analogy to the CPR structure, ACE-1 should be located on the external faces of the homodimer and binding sites would be expected to be freely accessible, whereas, ACE-2 is located at 
the dimer interface, ACE-2-derived peptides which may limit access to the interior face of the flavins where they may exert an inhibitory effect.

Structural information regarding the $\mathrm{C}$-terminus and the autoinhibitory domain will be essential to define the actual mode(s) of regulation carried out by these domains. However, this information may not be forthcoming given the highly mobile nature predicted for the elements involved.

\subsubsection{ACE-2 and "capping" of reductase activity}

The low rate of NO synthesis of $\mathrm{eNOS}(\approx 130 \mathrm{nmole} / \mathrm{min} / \mathrm{mg})$ compared to nNOS $(\approx 500 \mathrm{nmole} / \mathrm{min} / \mathrm{mg})$ or iNOS $(\approx 600 \mathrm{nmole} / \mathrm{min} / \mathrm{mg})$, has been demonstrated to be due to an attenuated activity of the eNOS reductase domain. Deletion of ACE-1 from eNOS restores activity to a level comparable to that of nNOS or iNOS, whereas, deletion of ACE-1 from nNOS does not influence catalytic rate. Hence, it was assumed that while the reductase domain of eNOS was "handicapped", those of nNOS and iNOS represented the maximum catalytic rates possible. However, deletion of ACE-2 in its entirety (21-42 amino acids, depending on isoform) caused an increase in the maximal rates of cytochrome $\mathrm{c}$ reduction in all isoforms. This observation suggests that even for iNOS and nNOS, the rate of electron flow is "capped", even in the "maximally active" CaM-bound form. Why would evolutionary forces act to "cap" the reductase activity of NOSs? The answer to this question for

cNOSs would seem to be for regulation by $\mathrm{Ca}^{2+} / \mathrm{CaM}$. The reductase activities of wildtype cNOSs are greatly potentiated by $\mathrm{CaM}$ binding, whereas, in the absence of ACE-2 reductase activities are already at or near maximal. For iNOS, the capping of 
electron flux does not provide for regulation by $\mathrm{Ca}^{2+} / \mathrm{CaM}$, but may instead provide a critical means to limit the production of superoxide from the reductase flavins (Xia $e t$ al., 1998). The rate-limiting step for NO synthesis of iNOS lacking the entirety of ACE-2 is in the oxygenase domain. Indeed, evolutionary pressure to prevent the simultaneous cellular generation of high-output NO and superoxide, and the resulting reactive products, would presumably be great. Thus, in the absence of ACE-2, the situation would favor the production of flavin-derived superoxide, due to a "backlog" of electron transport by the reductase domain. ACE-2 may act as a built in mechanism to stop this situation from arising, by limiting the maximal flux of electrons through the reductase domain to ensure the production of NO.

\subsubsection{ACE-2 and reductase $\rightarrow$ oxygenase electron transfer}

Although we have been discussing ACE-2 from an autoinhibitory point of view, certain aspects of the truncation mutant phenotype suggest that the presence of ACE-2 (or regions of it) is a requirement for efficient NO synthesis. Although, truncated cNOSs, lacking the entirety of ACE-2, exhibit a similar CaM-bound rate of electron flux through the reductase domain, compared to the relevant wildtype, this does not translate to NO synthesis. Indeed, truncated mutants exhibit only a $33-45 \%$ of the increase in activity observed in the cognate wildtype cNOS. This would indicate that ACE-2 is required for an efficient transfer of electrons from the reductase domain to the oxygenase domain. In effect, the presence of ACE-2 may serve to stabilize the FMN-heme contacts required for effective electron transfer. In the case of our mutant, lacking only the 27 amino acids at the C-terminal end of ACE2 , increased reductase activity is mirrored by an increased rate of NO synthesis. This would imply that the stability of FMN-heme contacts is maintained in this mutant, 
suggesting that regions of ACE-2, N-terminal to $\operatorname{Ser}_{1179}$, are specifically required to stabilize the contacts for efficient reductase $\rightarrow$ oxygenase electron transfer, and hence efficient NO synthesis. Indeed, there is a high degree of conservation in this region between eNOS (bovine $\mathrm{eNOS}_{1163-1178}$ ) and $\mathrm{nNOS}$ (rat $\mathrm{nNOS}_{1397-1429}$ ), suggesting an important functional role. For iNOS, truncation of ACE-2 did not compromise the ability of the mutant to synthesize NO, probably due to the tight "coupling" of FMNheme electron transfer contributed by additional structural features that are unique by this isoform.

\subsubsection{Binding of $\mathrm{CaM}$ and activation of eNOS are related, yet distinct processes}

It is commonly assumed that $\mathrm{CaM}$ binding to eNOS and enzyme activation are obligately linked events. On the contrary, with wildtype eNOS, we observe a large disparity between the $\mathrm{Ca}^{2+}$-dependence curves for CaM-binding and activation (see Fig. 4.16), a phenomenon previously observed for nNOS (B.A. Weissman, C.L. Jones, B.S. Masters, P. Martasek and S.S. Gross, submitted). Binding of CaM to eNOS is over $50 \%$ saturated at $\mathrm{Ca}^{2+}$ concentrations that support less than $5 \%$ of maximal NO synthesis. This would suggest that CaM could be bound to eNOS without eliciting activation of the enzyme.

A reasonable explanation for this disparity can be obtained from considering the structure of CaM itself (see Fig. 4.24). CaM consists of two lobes held together by a flexible linker domain. Upon binding of $\mathrm{Ca}^{2+}$, the lobes of $\mathrm{CaM}$ undergo a conformational change, which reveals sites required for interaction with target proteins. Notably, the two lobes of CaM are activated at very different levels of free 
$\mathrm{Ca}^{2+}$, the C-terminal lobe at $-100 \mathrm{nM}$ and the $\mathrm{N}$-terminal lobe at $\sim 700 \mathrm{nM}$ free $\mathrm{Ca}^{2+}$ (Persechini et al., 1996). It has previously been shown that binding of the $\mathrm{N}$-terminal lobe of CaM is responsible for the activation of nNOS (Persechini et al., 1996).

The large difference in $\mathrm{Ca}^{2+}$-affinity between the C- and N-lobes of CaM suggests that at low levels of $\mathrm{Ca}^{2+}(100-200 \mathrm{nM})$, only the high-affinity C-lobe EF hand pairs $\left(\mathrm{K}_{\mathrm{d}}<100 \mathrm{nM}\right)$ will bind $\mathrm{Ca}^{2+}$. Hence, a CaM species will exist with an activated $\mathrm{C}$-lobe and an inactive $\mathrm{N}$-lobe, a species that could potentially bind to eNOS without eliciting activation. The N-lobe EF hand pairs, having a 6-fold lower affinity for $\mathrm{Ca}^{2+}$, would not become active until much higher levels of intracellular $\mathrm{Ca}^{2+}$ were attained. Thus, when a stimulus-evoked $\mathrm{Ca}^{2+}$ spike is initiated, it is the $\mathrm{C}$-lobe that first undergoes the compaction process, exposing the acidic regions required for interaction with eNOS. As binding of the C-lobe is insufficient to elicit eNOS activation, an inactive eNOS-CaM complex could be formed, in which $\mathrm{CaM}$ is tethered to eNOS via the C-lobe. A further increase in the level of free $\mathrm{Ca}^{2+}$ would result in the compaction of the $\mathrm{N}$-lobe, binding of the $\mathrm{N}$-lobe to CaM, and enzyme activation.

From a physiological point of view, this mechanism would offer two desirable features: rapid response to a stimulus and prompt termination following cessation of the stimulus. Several properties of the CaM-cNOS interaction may contribute to the rapid on/off response. The inactive eNOS-CaM complex, with CaM bound solely via the $\mathrm{C}$-lobe, would hold the $\mathrm{N}$-lobe in close proximity to the $\mathrm{CaM}$ binding site, creating a very high local concentration of the N-lobe. This would shift the equilibrium towards the activation of eNOS. Additionally, once CaM is tethered to 
eNOS via the C-lobe, this may induce a conformational change in the N-lobe to increase the apparent affinity for $\mathrm{Ca}^{2+}$. Together, these factors would increase the efficiency of CaM-dependent activation during a transient increase in intracellular $\mathrm{Ca}^{2+}$ levels. The rapid activation of $\mathrm{cNOS}$ is enhanced by the fact that $\mathrm{Ca}^{2+}$-binding to the N-lobe EF hands of $\mathrm{CaM}$ occurs 70 times faster $\left(1.6 \times 10^{8} \mathrm{M}^{-1} \mathrm{~s}^{-1}\right)$ than binding to the $\mathrm{C}$-lobe EF hands $\left(2.3 \times 10^{6} \mathrm{M}^{-1} \mathrm{~s}^{-1}\right)$. This would provide for exceedingly rapid binding of the N-lobe of tethered $\mathrm{CaM}$, and hence cNOS activation, in response to a calcium-elevating stimulus. There is also evidence that $\mathrm{Ca}^{2+}$ dissociates much more quickly from the $\mathrm{N}$-lobe of $\mathrm{CaM}$, compared to the C-lobe (5 to 29-fold faster, for $\mathrm{Ca}^{2+} / \mathrm{CaM}$ complexes with myosin light chain kinase or CaM kinase (Johnson et al., 1996). Hence, as intracellular $\mathrm{Ca}^{2+}$ returns to resting levels, binding of the $\mathrm{N}$-lobe of CaM would be lost rapidly, resulting in acute shut-down of NO synthesis.

\subsubsection{A physiological model of eNOS activation}

From consideration of the range of intracellular $\mathrm{Ca}^{2+}$ levels incurred during a stimulus-evoked $\mathrm{Ca}^{2+}$-spike, a physiological model can be constructed. Resting levels of intracellular $\mathrm{Ca}^{2+}$ are approximately $100 \mathrm{nM}$, which can be increased to as high as 1-2 $\mu \mathrm{M}$ upon stimulation. The C-lobe of CaM, with an apparent $\mathrm{K}_{\mathrm{d}}$ of less than 100 $\mathrm{nM} \mathrm{Ca}{ }^{2+}$, would be expected to have a high degree of $\mathrm{Ca}^{2+}$-occupancy even at resting levels of intracellular $\mathrm{Ca}^{2+}$. Thus, it may be erroneous to think of $\mathrm{CaM}$ as "free floating " in the endothelial or neuronal cell where it awaits cNOS interaction upon transient stimulation-evoked increases in intracellular $\mathrm{Ca}^{2+}$ levels. Instead, it is likely that $\mathrm{CaM}$ is to a significant extent pre-bound through its $\mathrm{C}$-lobe to $\mathrm{cNOSs}$ at resting 
levels of intracellular $\mathrm{Ca}^{2+}$, in a conformation that does not elicit enzyme activation. Subsequent stimulation-evoked increases in intracellular $\mathrm{Ca}^{2+}$ would then cause the $\mathrm{N}$-terminal lobe of $\mathrm{CaM}$ to compact and assume the conformation required for $\mathrm{cNOS}$ binding and activation. Thus, a two-stage model for $\mathrm{Ca}^{2+} / \mathrm{CaM}$ activation of $\mathrm{cNOSs}$ is suggested wherein CaM-binding occurs at one $\mathrm{Ca}^{2+}$ concentration and activation of cNOS only occurs at a higher $\mathrm{Ca}^{2+}$ concentration. This would be similar to the proposed mode of activation of phosphorylase kinase, $\mathrm{a} \mathrm{Ca}^{2+} / \mathrm{CaM}$-dependent protein kinase in which $\mathrm{CaM}$ is present as a tightly bound subunit regardless of activation state (Cohen et al., 1978; Grand et al., 1981). The resulting interaction of the N-lobe of $\mathrm{CaM}$ with the $\mathrm{C}$-terminal aspect of the $\mathrm{CaM}$ binding site on cNOSs would potentially displace the adjacent ACE-1 or the C-terminal ACE-2, and contribute to activation, as discussed later.

The activation of myosin light chain kinase (MLCK), a $\mathrm{Ca}^{2+} / \mathrm{CaM}$-dependent kinase, was the subject of a recent small angle scattering study (Krueger et al., 1998) that is particularly relevant to the model we propose for the activation of eNOS. The author demonstrated that $\mathrm{CaM}$ binding to MLCK begins at substoichiometric $\mathrm{Ca}^{2+}$ concentrations, i.e. when only $\mathrm{C}$-terminal $\mathrm{EF}$ hands have bound $\mathrm{Ca}^{2+}$. Once bound via the $\mathrm{C}$-lobe, a further increase in $\mathrm{Ca}^{2+}$ levels resulted in $\mathrm{Ca}^{2+}$ binding at the $\mathrm{N}$-lobe $\mathrm{EF}$ hands of CaM, eliciting MLCK activation. Binding of CaM to MLCK solely via the $\mathrm{C}$-lobe was confirmed by the use of a mutant $\mathrm{CaM}$, in which the $\mathrm{N}$-terminal $\mathrm{EF}$ ands were incapable of binding $\mathrm{Ca}^{2+}$. This study also concluded that, at resting levels of intracellular $\mathrm{Ca}^{2+}$, CaM would likely be tethered to MLCK via the C-lobe in an inactive complex. This complex is then "primed" for rapid response to a stimulusevoked increase in intracellular $\mathrm{Ca}^{2+}$. This model for MLCK activation by $\mathrm{Ca}^{2+} / \mathrm{CaM}$ 
is essentially identical to the one we propose for the activation of eNOS in vivo, although our conclusion derives from a completely different line of investigation.

We acknowledge that the model for the physiological activation of eNOS proposed above, is presented in its simplest form and does not take into account additional cellular variables that may be important, such as concentration of $\mathrm{Mg}^{2+}$ ions, caveolins or HSP90.

The model is summarized below and shown in Fig. 4.25.

A three-stage model for the activation of eNOS by $\mathrm{Ca}^{2+} / \mathrm{CaM}$

The following information suggests a sequential multi-step model for the $\mathrm{Ca}^{2+} / \mathrm{CaM}$-dependent regulation of eNOS (in accord with our previous model for nNOS activation):

a) CaM binds to eNOS at subsaturating levels of $\mathrm{Ca}^{2+}$

b) CC-CaM binds to eNOS at subsaturating levels of $\mathrm{Ca}^{2+}$, but elicits no activation

c) Binding of the N-terminal lobe of CaM is essential for wildtype eNOS activation, but not $\mathrm{C} \Delta 27$ eNOS activation.

Using these facts, we propose this three-stage model for eNOS activation:

1) In a resting cell $\left(100 \mathrm{nM} \mathrm{Ca}^{2+}\right), \mathrm{CaM}$ exists predominantly with two $\mathrm{Ca}^{2+}$ ions bound to the C-lobe EF-hand pairs. The C-lobe is therefore compacted and primed for binding to eNOS. The compacted C-lobe interacts with the CaM-binding site of eNOS, but the enzyme remains inactive. 
2) A stimulus-evoked increase in intracellular $\mathrm{Ca}^{2+}$ (approaching or exceeding 1 $\mu \mathrm{M})$ triggers the binding of $\mathrm{Ca}^{2+}$ to the two remaining EF-hands of CaM-causing compaction of the N-lobe, however, eNOS still remains inactive.

3) The compacted N-lobe of eNOS-bound CaM binds to its cognate binding site, triggering full activation of eNOS.

\subsubsection{ACE-2 and $\mathrm{Ca}^{2+} / \mathrm{CaM}$-dependence of eNOS activation}

Truncation of ACE-2 resulted in a 5 to 6-fold reduction in $\mathrm{EC}_{50}$ for $\mathrm{Ca}^{2+}$ required for the activation of eNOS by CaM, compared to wildtype. Since removal of $\mathrm{ACE}-2$ did not directly affect the $\mathrm{Ca}^{2+}$-dependence or affinity of $\mathrm{CaM}$ for binding, these effects must be due to differences exhibited by the inactive CaM-eNOS complex. Given the model outlined in the previous section, at low levels of free- $\mathrm{Ca}^{2+}$, CaM would be bound to eNOS via the C-lobe only. For C $\Delta 27$ eNOS to exhibit activity at low concentrations of free $\mathrm{Ca}^{2+}$, either the interactions between the $\mathrm{C}$-lobe of $\mathrm{CaM}$ and eNOS are sufficient to elicit activation or once tethered to $\mathrm{C} \triangle 27$ eNOS by the $\mathrm{C}$-lobe, the affinity of the $\mathrm{N}$-lobe for $\mathrm{Ca}^{2+}$ becomes increased.

From our experiments with $\mathrm{CC}-\mathrm{CaM}$ it seems that there are subtle differences in the mechanism of CaM-dependent regulation between wildtype and CA27 eNOS. Although CC-CaM binds to wildtype eNOS, it does not initiate NO synthesis. Furthermore, binding of CC-CaM does not evoke any significant increase in either of the measured reductase activities of wildtype eNOS. However, CC-CaM does elicit partial activation of $\mathrm{C} \Delta 27 \mathrm{eNOS}$, to approximately $30 \%$ of that elicited by wildtype 
Fig. 4.24. Structure of CaM based on X-ray diffraction studies. Taken from "Molecular Biology of the Cell", $2^{\text {nd }}$ Edition, p711, by B. Alberts, et al. 

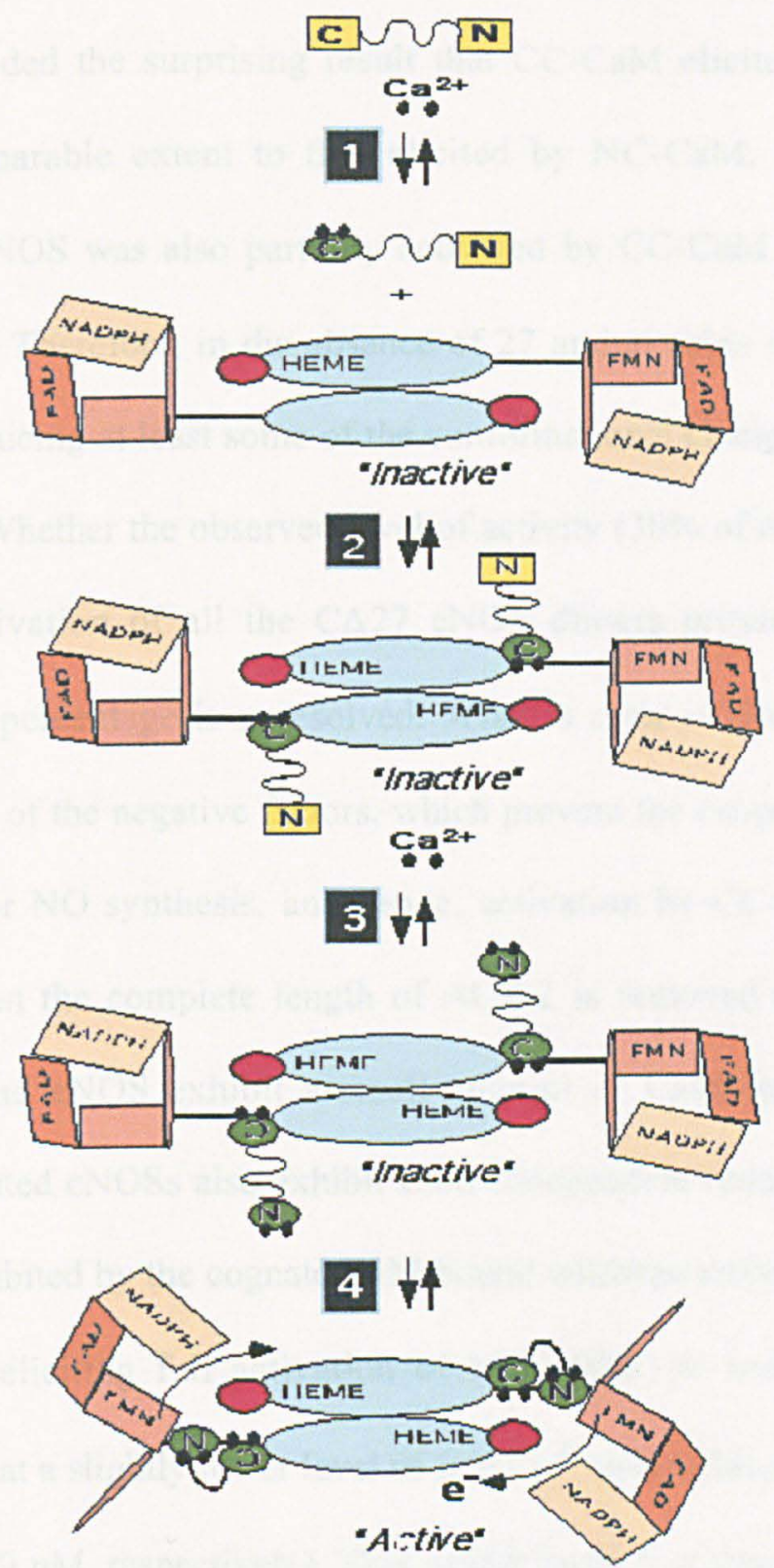

Fig. 4.25. Four-Step model of eNOS activation by $\mathrm{Ca}^{2+} / \mathrm{CaM}$. (1) In a resting cell $\left(100 \mathrm{nM} \mathrm{Ca}^{2+}\right), \mathrm{CaM}$ exists with two $\mathrm{Ca}^{2+}$ ions bound to the $\mathrm{C}$-lobe, the $\mathrm{C}$-lobe is therefore compacted and primed for binding to eNOS. (2) The compacted C-lobe interacts with the CaM-binding site of eNOS, but the enzyme remains inactive. (3) A stimulus-evoked increase in intracellular $\mathrm{Ca}^{2+}(\leq 1 \mu \mathrm{M})$ causes the binding of $\mathrm{Ca}^{2+}$ to the two remaining EF-hands of CaM-causing compaction of the N-lobe. However, eNOS remains inactive. (4) Binding of the compacted N-lobe of CaM to eNOS elicits full activation. 
CaM (NC-CaM). Studies of the ability of CC-CaM to activate the reductase functions of $\mathrm{C} \triangle 27$ eNOS provided the surprising result that $\mathrm{CC}-\mathrm{CaM}$ elicited ferricyanide reduction to a comparable extent to that elicited by NC-CaM. Cytochrome c reduction by $\mathrm{C} \Delta 27$ eNOS was also partially activated by $\mathrm{CC}-\mathrm{CaM}, \approx 20 \%$ of that elicited by NC-CaM. Therefore, in the absence of 27 amino acids of ACE-2, CCCaM is capable of inducing at least some of the conformational changes required for the synthesis of NO. Whether the observed level of activity (30\% of maximal) is due to a submaximal activation of all the $\mathrm{C} \Delta 27$ eNOS dimers present or maximal activation of a small percentage is unresolved. What is clear is that truncation of ACE-2 removes some of the negative factors, which prevent the proper alignment of co-factors required for NO synthesis, and hence, activation by CC-CaM becomes feasible. Indeed, When the complete length of ACE-2 is removed (Roman et al., 2000), both nNOS and eNOS exhibit a small amount of CaM-independent NO synthesis. Such truncated cNOSs also exhibit CaM-independent reductase functions in excess of those exhibited by the cognate CaM-bound wildtype enzymes. NN-CaM, which is capable of eliciting full activation of both wildtype and $C \Delta 27$ eNOS, activates $\mathrm{C} \triangle 27$ eNOS at a slightly lower level of free $\mathrm{Ca}^{2+}$ than wildtype eNOS $\left(\mathrm{EC}_{50}\right.$ values of 853 and $1089 \mathrm{nM}$, respectively). This would imply that truncation of ACE2 , removes an obstacle to the binding of the $\mathrm{N}$-lobe of $\mathrm{CaM}$, allowing activation at lower levels of free $\mathrm{Ca}^{2+}$. 
ACE-2 (eNOS $1163-1205)$ has previously been implicated in eNOS regulation, but in another context. Indeed, phosphorylation of Ser 1179 (bovine sequence, see Fig.4.26) by Akt or PKA, has been demonstrated to be a factor in the in vivo activation of eNOS by physiological stimuli (Dimmeler et al., 2000; Dimmeler et al., 1999; Fisslthaler et al., 2000; Fulton et al., 1999; Luo et al., 2000). Once phosphorylated at this residue, eNOS exhibits a 2 to 3 -fold increase in catalytic rate and demonstrates activity even at resting levels of intracellular $\mathrm{Ca}^{2+}$. Analysis of our truncated protein provides some insights into the underlying mechanism of eNOS regulation by phosphorylation of $\operatorname{Ser}_{1179}$. C $\Delta 27$ eNOS, lacks Ser $_{1179}$, and all amino acids C-terminal to it, and exhibits a similar increase in catalytic rates to that observed for phosphorylated wildtype eNOS. Thus, removal of 27 amino acids from the Cterminus (including Ser $_{1179}$ ) mimics the effects of phosphorylation of $\operatorname{Ser}_{1179}$. This would imply that phosphorylation of this residue represents a "loss of function" rather than a "gain of function". Given the regulatory function assigned to the C-terminus (ACE-2) in limiting maximal activity of the enzyme, phosphorylation of $\operatorname{Ser}_{1179}$ probably removes/diminishes the autoinhibitory effects of ACE-2. The manner by which phosphorylation of Ser 1179 diminishes the inhibitory effects of ACE-2 is also unknown. However, it is conceivable that the incorporation of a phosphate at this position may cause a conformational change by repulsion/attraction of neighboring residues that offsets the interactions which sustain tonic inhibition.

Phosphorylation of Ser 1179 also allows " $\mathrm{Ca}^{2+}$-independent" activation of eNOS when transfected into cultured ECV304 cells (Fulton et al., 1999). However, in cell- 
free assays, neither phosphorylated eNOS nor eNOS mutants mimicking this phosphorylation ( $\operatorname{Ser}_{1179} \rightarrow$ Asp $_{1179}$ ), exhibit a significant reduction in the $\mathrm{EC}_{50}$ for $\mathrm{Ca}^{2+}$ required for NO synthesis. Interestingly, $\mathrm{C} \Delta 27$ eNOS exhibits a 5 to 6 -fold decrease in the $\mathrm{EC}_{50}$ for $\mathrm{Ca}^{2+}$ required for activity and eNOS lacking $\mathrm{ACE}-2$ in its entirety, exhibit a degree of activity even in the absence of bound $\mathrm{CaM}$. This disparity in relative $\mathrm{Ca}^{2+}$-dependence between in vitro and in vivo situations suggests that other modifications, in addition to phosphorylation of $\mathrm{Ser}_{1179}$, are required for eNOS to exhibit " $\mathrm{Ca}^{2+}$-independent" activity in cells. The complete removal of this region of eNOS, as in C $\triangle 27$ eNOS or similar truncations, mimics the effects of these additional interactions. As eNOS activation has been shown to involve an interaction with HSP90, an attractive possibility is that HSP90 stabilizes a conformation in phosphorylated eNOS, which entirely negates the effects of ACE-2 and allows " $\mathrm{Ca}^{2+}$ independent activation".

Although their can be little doubt that eNOS is regulated in vivo by Aktmediated phosphorylation, it seems that this site is also conserved within ACE-2 regions of the nNOS isoforms ( $\operatorname{Ser}_{1412}$ in the rat sequence, see Fig. 4.25). Although no functional significance has been demonstrated for this site, it would be an attractive mechanism for " $\mathrm{Ca}^{2+}$-independent" activation of nNOS. However, stimuli that evoke such a " $\mathrm{Ca}^{2+}$-independent" activation of nNOS, have yet to be identified. However, evidence now points to a role for Akt-mediated phosphorylation in the $\mathrm{Ca}^{2+}$. dependent activation of eNOS, a similar mode of activation is not unlikely for nNOS. 


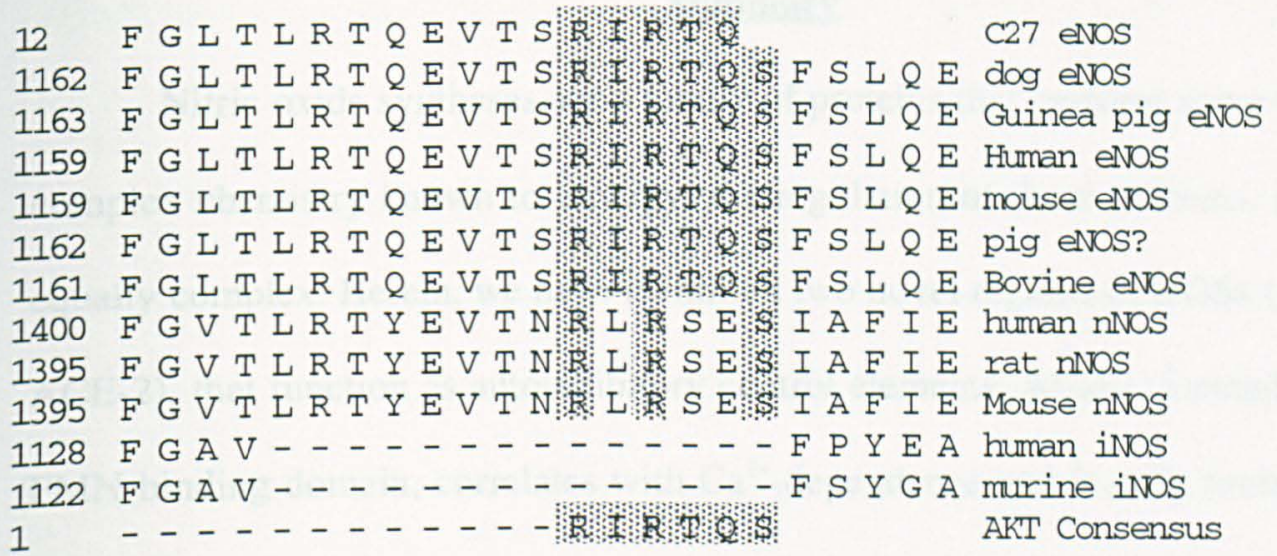

Fig. 4.26. Conservation of the Akt phosphorylation site throughout the cNOSs. The Akt site is perfectly conserved throughout the eNOS family of proteins. Additionally, although no functional significance has been demonstrated, the Akt site is also present in members of the nNOS family of proteins. 


\section{Chapter 5}

\section{Summary}

Nitric oxide synthases are a family of proteins that perform some of the most complex chemistry known to biology; the regulation of these proteins, it seems, is equally complex. Herein, we have identified two novel regions of NOSs (ACE-1 and ACE-2), that function as autoinhibitory control elements. ACE-1, located within the FMN-binding domain, correlates with $\mathrm{Ca}^{2+}$-dependence and is only found in eNOS and nNOS. The presence of ACE-1, largely accounts for the observed $\mathrm{Ca}^{2+}$ dependence of the cNOSs and serves to govern the reductase activities of eNOS. ACE-2, located within an extension of the C-terminus, is present in all the isoforms of NOS. However, regions of ACE-2 are unique to, and conserved in, cNOSs. Removal of 27 amino acids of ACE-2 causes an increased catalytic rate and reduces the $\mathrm{Ca}^{2+}$ requirement for maximal activity. Removal of ACE- 2 in its entirety causes a shift to a CaM-independent NO synthesis, albeit much less efficiently than that of the CaMbound. The molecular basis for the function of these regions in the regulation of NOS is unknown. Structural information pertaining to the reductase domain of cNOSs will be required to elucidate their function, however, due to the highly mobile status of the ACEs, acquisition of such structural information may not be forthcoming.

\subsection{ACE-1. ACE-2 and the CaM-binding Site: A tripartate system for regulation of} cNOS.

From my work, it seems that there are three major regions responsible for the regulation of eNOS, the CaM-binding site, ACE-1 and ACE-2. From the work of others, it is evident that this is also the case for nNOS. Indeed, iNOS, may also have this trifecta of regulatory regions, although it lacks ACE-2, its high affinity CaM- 
binding can only be explained by the presence of auxiliary CaM-binding regions (which may reside in a position analogous to ACE-1, see discussion Chapter 3).

This trifecta of regulatory regions works in concert to facilitate the $\mathrm{Ca}^{2+} / \mathrm{CaM}$ dependent activation/inactivation of eNOS, in response to physiological stimuli. The CaM-binding site, quite obviously, binds to $\mathrm{Ca}^{2+} / \mathrm{CaM}$. $\mathrm{ACE}-1$ acts as a steric impediment to $\mathrm{Ca}^{2+} / \mathrm{CaM}$-binding and is displaced during the CaM-binding process. ACE-2 acts as a gate to electron flow between the NOS co-factors (NADPH $\rightarrow$ FAD $\rightarrow$ FMN $\rightarrow$ Heme), the negative modulatory effects of ACE-2 are alleviated by $\mathrm{Ca}^{2+} / \mathrm{CaM}$-induced conformational changes.

The following scheme of eNOS regulation, derived from interpretation of our data, and that of others (Daff et al., 1999; Dimmeler et al., 1999; Fulton et al., 1999; Montgomery et al., 2000; Nishida \& Ortiz de Montellano, 1999; Nishida \& Ortiz de Montellano, 1998; Roman et al., 2000; Roman et al., 2000), offers a possible mechanism for the tripartate regulation mentioned previously. Regulation of eNOS is accomplished by a conspiracy between $\mathrm{Ca}^{2+} / \mathrm{CaM}, \mathrm{ACE}-1$ and ACE-2. The ACEs exert negative effects which must be alleviated in order to elicit eNOS activation. In the absence of bound $\mathrm{CaM}, \mathrm{ACE}-1$ occupies as yet unknown regulatory binding sites. ACE-2, we propose to exert effects which push the NADPH/FAD/FMN alignment out of alignment, thereby limiting the flow of electrons through the reductase domain. Following a stimulus-evoked increase in intracellular $\mathrm{Ca}^{2+}$, the C-terminal lobe of CaM becomes compacted and binds to eNOS, however, eNOS does not become activated. A further increase in intracellular $\mathrm{Ca}^{2+}$ causes the compaction of the $\mathrm{N}$ terminal lobe of CaM. The subsequent interaction between the $\mathrm{N}$-lobe of $\mathrm{CaM}$ and the 
C-terminal portion of the CaM-binding site of eNOS, displaces ACE-1 from its regulatory sites. $\mathrm{N}$-lobe binding, displacement of $\mathrm{ACE}-1$, or more likely a combination of the two, overcome the tonic inhibitory effects exerted by ACE-2. This allows proper alignment of the flavins for efficient electron transfer. The yin/yang relationship between ACE-2, which is envisioned to push the flavins out of alignment and $\mathrm{Ca}^{2+} / \mathrm{CaM}$, which serves to push the flavins into alignment, may provide the "molecular toggle" by which $\mathrm{Ca}^{2+} / \mathrm{CaM}$ elicits activation. $\mathrm{ACE}-1$ may serve simply as a hindrance to the binding of $\mathrm{Ca}^{2+} / \mathrm{CaM}$, thus prolonging the effects of ACE-2. Thus deletion of ACE-1 may simply remove this obstacle, providing $\mathrm{Ca}^{2+} / \mathrm{CaM}$ binding at lower levels of free $\mathrm{Ca}^{2+}$ and favoring the alleviation of the inhibition exerted by ACE-2. Another explanation would be a direct interaction between the two ACEs, however, by analogy to the CPR structure, ACE-1 and ACE-2 would appear to reside on opposite sides of the NOS monomer, diminishing the likelihood for direct interaction.

$\mathrm{Ca}^{2+}$-independent activation of eNOS is explained by Akt-mediated phosphorylation of $\mathrm{Ser}_{1179}$; our results indicate that this posttranslational modification of eNOS removes/reduces the inhibitory effects exerted by ACE-2. This would then allow eNOS to be active at resting levels of intracellular $\mathrm{Ca}^{2+}$. Activation of phosphorylated eNOS at resting levels of intracellular $\mathrm{Ca}^{2+}$ may only require binding of the C-lobe of CaM. Our C $\Delta 27$ eNOS truncation was partially activated by an engineered $\mathrm{CaM}$, which expressed two $\mathrm{C}$-lobes. This process may not require the displacement of ACE-1, which we predict to interfere with binding of the N-lobe of CaM. From the study of mutants mimicking the phosphorylation of $\operatorname{Ser}_{1179}$, it is likely that " $\mathrm{Ca}^{2+}$-independent" activation of eNOS requires an association with protein(s) 
other than CaM. Given its established role in the regulation of eNOS, a good candidate for such an "other" interacting protein would be HSP90 (Garcia-Cardena et al., 1998).

Obviously, the regulation of eNOS is multifactorial in its complexity. In the above model, we have not accounted for the various regulatory modifications eNOS undergoes in vivo. The regulation of eNOS, much more so than other NOS isoforms, involves reversible palmitoylation in addition to multiple protein-protein interactions. Inhibitory complexes of eNOS with members of the caveolin family of proteins (Garcia-Cardena et al., 1996; Garcia-Cardena et al., 1997; Ju et al., 1997; Michel et al., 1997; Venema et al., 1997) and members of the transmembrane receptors (Ju et al., 1998) have been reported. In addition, activated eNOS has been found to form a complex with HSP90 that enhances maximal NO production (Garcia-Cardena et al., 1998). These factors all contribute to multifactorial regulation of eNOS, that is superimposed on the simple model we present. 


\section{Chapter 6}

\section{Future Work}

\subsection{ACE-1/ACE-2 interplay}

Given the similar phenotypes of eNOS mutants lacking ACE-1 or ACE-2, and that a mutant lacking a region of ACE- 2 is a resistant to inhibition by ACE- 1 derived peptides, we proposed an interaction between the two ACE regions. If ACE-2 contained the binding site for ACE-1, then removal of either one would negate the effect of the other. We propose to investigate this in two ways. Firstly, using a fluorescein-tagged ACE-1 derived peptide $\left(\mathrm{eNOS}_{628-634}\right)$, we would investigate the interaction of this peptide with wildtype $\left(\mathrm{eNOS}_{1-1205}\right), \mathrm{C} \Delta 27\left(\mathrm{eNOS}_{1-1178}\right)$ and a truncation mutant of eNOS lacking the entirety of ACE-2 (eNOS $1-1163)$. If the binding site of ACE-1 is contained within ACE-2, then the truncated proteins would be expected to exhibit a lower affinity for binding of the fluorescently tagged protein. Relative affinity would be assessed in a 96-well based assay using a microplate fluorometer. Secondly, additional mutagenesis would be performed to express eNOS mutants lacking the entirety of ACE-1, the entirety of ACE-2, the entirety of ACE-1 and ACE-2, and C $\triangle 27$ lacking the entirety of ACE-1. By comparison of the phenotypes of these various mutants, the possibility of an interaction between the two could be ascertained. If the effects of ACE- 1 and ACE- 2 are mediated by distinct mechanisms, then the double deletion would be expected to exhibit a much higher level of activity than ether of the single deletions. If ACE-2 is the binding site of ACE-1, then deletion of either one would be equivalent to deletion of both. 


\subsection{Caveolin and $\mathrm{Ca}^{2+} / \mathrm{CaM}$}

One of the possibilities we put forward is that CaM may be tethered to eNOS at resting levels of intracellular $\mathrm{Ca}^{2+}$, by interactions between eNOS and the C-lobe of CaM. The basis for this was the high affinity of the C-lobe for $\mathrm{Ca}^{2+}\left(\mathrm{K}_{d}<100 \mathrm{nM}\right)$ (Persechini et al., 1996; Persechini et al., 1996) combined with the established values for resting levels of intracellular $\mathrm{Ca}^{2+}$ (Nathan \& Xie, 1994). However, the interaction of eNOS with caveolin was not considered in this hypothesis. Caveolin and CaM seem to bind to eNOS in a competitive manner; inactive eNOS exists as a membranebound complex with caveolin-1 (Cav-1) (Garcia-Cardena et al., 1996; Garcia-Cardena et al., 1997; Ju et al., 1997; Michel et al., 1997; Venema et al., 1997) which dissociates upon activation by $\mathrm{Ca}^{2+} / \mathrm{CaM}$ the eNOS-Cav- 1 . However, from the numerous experiments carried out thus far, it is unclear whether it is possible for the C-lobe of CaM and Cav-1 to simultaneously bind to eNOS. Most in vitro experiments have considered the interaction between eNOS and Cav-1 (or derived peptides) solely for its effect on eNOS activity. CaV-1 mediated inhibition of eNOS activation was fully reversible by excess $\mathrm{CaM}$, but his does not preclude the existence of a three part, eNOS-Cav-1-CaM (bound only by the C-lobe) interaction. To investigate this possibility, we would use the engineered mutant CaM protein expressing two C-lobes (CC-CaM) in conjunction with Cav-1-glutathione-s-transferase (GST) fusion protein. By using Cav-1-GST fusion protein bound to glutathione-agarose beads, the relative effects of wildtype and CC-CaM on Cav-1-eNOS complex dissociation could be assessed using the established "pull-down" method. 


\subsection{HSP90 and phosphorylation of ACE-2}

Mutants eNOSs mimicking the phosphorylation of $\mathrm{Ser}_{1179}$, exhibit " $\mathrm{Ca}^{2+}-$ independent" activity when transfected into cells. However, when the $\mathrm{Ca}^{2+}$ dependence is assessed in vitro, activity at such low levels of $\mathrm{Ca}^{2+}(100 \mathrm{nM})$ are not observed (McCabe et al., 2000). This suggests that phosphorylation of eNOS, by itself, is insufficient to elicit activation of eNOS at resting levels of intracellular $\mathrm{Ca}^{2+}$. This indicated that an intracellular factor, perhaps HSP90, may be crucial to facilitate the apparent " $\mathrm{Ca}^{2+}$-independent" activity of eNOS. We propose that HSP90 might stabilize a conformation of phosphorylated eNOS, which fully alleviates the inhibitory effects of ACE-2 and allows " $\mathrm{Ca}^{2+}$-independent" activation of eNOS. To test this we propose an in vitro reconstitution experiment using recombinant HSP90. The ability of Akt-mediated phosphorylation to elicit " $\mathrm{Ca}^{2+}$-independent" activation of eNOS shall be addressed in two ways. Firstly, in vitro phosphorylation of eNOS using recombinant Akt (Upstate Biotech, Lake Placid, NY) will be used. Secondly, mutation of $\operatorname{Ser}_{1179}$ to mimic phosphorylation of this residue (McCabe et al., 2000) will be carried out. The $\mathrm{Ca}^{2+}$-dependence curves of these enzymes shall then be compared to wildtype eNOS, in the absence or presence of an equal amount of HSP90. If HSP90 is the intracellular factor facilitating in vivo eNOS activity at resting levels of intracellular $\mathrm{Ca}^{2+}$, then phosphorylated/phosphorylation mimic eNOSs would be expected to exhibit activity at reduced levels of $\mathrm{Ca}^{2+}$ in the presence of HSP90. The techniques used here are described in Methods section.

\subsection{HSP 90 and $\mathrm{Ca}^{2+} / \mathrm{CaM}$ binding}

Although HSP90 elicits the activation of eNOS at resting levels of intracellular $\mathrm{Ca}^{2+}$, the underlying mechanism of this process is not well understood. 
One possibility is that once complexed with HSP90, the interaction between CaM and eNOS is stabilized, even at low levels of $\mathrm{Ca}^{2+}$. The effects of HSP90-eNOS complex formation on the stability of eNOS- $\left[{ }^{125} \mathrm{I}\right]-\mathrm{CaM}-\mathrm{eNOS}$ complexes will be assessed using the pre-incubation protocol outlined in Methods. Wildtype eNOS-[25I]-CaM complexes will be allowed to form in the presence or absence of recombinant HSP90. The complexes will then be transferred to EGTA/Ca ${ }^{2+}$ buffered solutions representing a range of $\mathrm{Ca}^{2+}$ concentrations. The level of $\left[{ }^{125} \mathrm{I}\right]-\mathrm{CaM}$ binding after a defined time period will then be assessed by scintillation counting. Additionally the rate of CaM dissociation of $\left[{ }^{125} \mathrm{I}\right]-\mathrm{CaM}$-eNOS complexes, compared to HSP90-[ $\left.{ }^{125} \mathrm{I}\right]-\mathrm{CaM}-\mathrm{eNOS}$ complexes will be assessed as described in Methods. If the HSP90-eNOS-CaM complex is significantly more stable than the eNOS-CaM complex, then this "complex stabilization" may provide a mechanism for the in vivo effects of HSP90 on the regulation of eNOS.

\subsection{Evaluation of Mutant eNOSs in Cultured Cells}

Although we have thoroughly characterized the various eNOS mutants in an in vitro setting, the behavior of such proteins in a cellular environment may provide insights into physiological eNOS regulation. This work is already underway, mutant protein cDNA (C $\triangle 27$, RRKRK sub, RRKRK del, $\mathrm{Glu}_{635}$ and wildtype eNOS) have been generated in a mammalian expression vector (pCDNA3, Invitrogen, Carlsbad, CA) for future transfection into cultured cells. Mammalian transfections would use two eNOS forms. Firstly, eNOSs incorporating a mutation of the myristoylation site $\left(\mathrm{Gly}_{2} \rightarrow \mathrm{Ala}_{2}\right)$, to allow cytosolic expression (Liu \& Sessa, 1994), will be transfected into a human embryonic kidney cell line (HEK 293). This will allow assessment of the mutants in the absence of complicating factors such as caveolin that would only apply to membrane-bound eNOS. Additionally, the effects of Caveolin/Akt/PKA etc 
could be assessed by co-transfection. Secondly, myristoylation-intact eNOSs, will be transfected into immortalized human bone endothelial cells (HBECs). This will permit the study of mutant eNOSs in their native cell type and presumably subcellular setting, effects of these mutations on regulation/interactions of eNOS could be studied in a more physiological setting. 


\section{Chapter 7}

\section{References}

Abu-Soud, H.M. \& Stuehr, D.J. (1993). Nitric oxide synthases reveal a role for calmodulin in controlling electron transfer. Proc Natl Acad Sci U S A, 90, 10769-72.

Abu-Soud, H.M., Wang, J., Rousseau, D.L., Fukuto, J.M., Ignarro, L.J. \& Stuehr, D.J. (1995). Neuronal nitric oxide synthase self-inactivates by forming a ferrous-nitrosyl complex during aerobic catalysis. J Biol Chem, 270, 22997-3006.

Abu-Soud, H.M., Yoho, L.L. \& Stuehr, D.J. (1994). Calmodulin controls neuronal nitric-oxide synthase by a dual mechanism. Activation of intra- and interdomain electron transfer. J Biol Chem, 269, 32047-50.

Adak, S., Ghosh, S., Abu-Soud, H.M. \& Stuehr, D.J. (1999). Role of reductase domain cluster 1 acidic residues in neuronal nitric-oxide synthase. Characterization of the FMN-FREE enzyme. J Biol Chem, 274, 22313-20.

Aisaka, K., Gross, S., Griffith, O. \& Levi, R. (1989). L-arginine availability determines the duration of acetylcholine-induced systemic vasodilation in vivo. Biochem Biophys Res Commun, 163, 710-17.

Albina, J.E., Cui, S., Mateo, R.B. \& Reichner, J.S. (1993). Nitric oxide-mediated apoptosis in murine peritoneal macrophages. J Immunol, 150, 5080-5.

Anagli, J., Hofmann, F., Quadroni, M., Vorherr, T. \& Carafoli, E. (1995). The calmodulin-binding domain of the inducible (macrophage) nitric oxide synthase. Eur J Biochem, 233, 701-8.

Anderson, R.G. (1998). The caveolae membrane system. Annu Rev Biochem, 67, $199-$ 225.

Anderson, R.G. (1993). Caveolae: where incoming and outgoing messengers meet. Proc Natl Acad Sci U S A, 90, 10909-13.

Andronik-Lion, V., Boucher, J.L., Delaforge, M., Henry, Y. \& Mansuy, D. (1992). Formation of nitric oxide by cytochrome P450-catalyzed oxidation of aromatic amidoximes. Biochem Biophys Res Commun, 185, 452-8.

Ankarcrona, M., Dypbukt, J.M., Brune, B. \& Nicotera, P. (1994). Interleukin-1 betainduced nitric oxide production activates apoptosis in pancreatic RINm5F cells. Exp Cell Res, 213, 172-7.

Argiolas, A. (1994). Nitric oxide is a central mediator of penile erection. Neuropharmacology, 33, 1339-44.

Arnet, U.A., McMillan, A., Dinerman, J.L., Ballermann, B. \& Lowenstein, C.J. (1996). Regulation of endothelial nitric-oxide synthase during hypoxia. J Biol Chem, 271, 15069-73. 
Arnold, W.P., Mittal, C.K., Katsuki, S. \& Murad, F. (1977). Nitric oxide activates guanylate cyclase and increases guanosine $3^{\prime}: 5$ '-cyclic monophosphate levels in various tissue preparations. Proc Natl Acad Sci U S A, 74, 3203-7.

Ayajiki, K., Kindermann, M., Hecker, M., Fleming, I. \& Busse, R. (1996). Intracellular $\mathrm{pH}$ and tyrosine phosphorylation but not calcium determine shear stressinduced nitric oxide production in native endothelial cells. Circ Res, 78, 750-8.

Ayoubi, T.A. \& Van De Ven, W.J. (1996). Regulation of gene expression by alternative promoters. Faseb J, 10, 453-60.

Azuma, H., Ishikawa, M. \& Sekizaki, S. (1986). Endothelium-dependent inhibition of platelet aggregation. Br J Pharmacol, 88, 411-5.

Babu, Y.S., Bugg, C.E. \& Cook, W.J. (1988). Structure of calmodulin refined at 2.2 A resolution. J Mol Biol, 204, 191-204.

Baek, K.J., Thiel, B.A., Lucas, S. \& Stuehr, D.J. (1993). Macrophage nitric oxide synthase subunits. Purification, characterization, and role of prosthetic groups and substrate in regulating their association into a dimeric enzyme. J Biol Chem, 268, 21120-9.

Beasley, D. \& Eldridge, M. (1994). Interleukin-1 beta and tumor necrosis factor-alpha synergistically induce NO synthase in rat vascular smooth muscle cells. Am J Physiol, 266, R1197-203.

Beauvais, F., Michel, L. \& Dubertret, L. (1995). The nitric oxide donors, azide and hydroxylamine, inhibit the programmed cell death of cytokine-deprived human eosinophils. FEBS Lett, 361, 229-32.

Beckman, J., Beckman, T., Chen, J., Marshall, P. \& Freeman, B. (1990). Apparent hydroxyl radical formation by peroxynitrite: implications for endothelial injury from nitric oxide and superoxide. Proc Nat Acad Sci, 87, 1620-24.

Bellmann, K., Jaattela, M., Wissing, D., Burkart, V. \& Kolb, H. (1996). Heat shock protein hsp70 overexpression confers resistance against nitric oxide. FEBS Lett, 391, 185-8.

Berlett, B.S., Friguet, B., Yim, M.B., Chock, P.B. \& Stadtman, E.R. (1996). Peroxynitrite-mediated nitration of tyrosine residues in Escherichia coli glutamine synthetase mimics adenylylation: relevance to signal transduction. Proc Natl Acad Sci US A, 93, 1776-80.

Billiar, T.R., Curran, R.D., Ferrari, F.K., Williams, D.L. \& Simmons, R.L. (1990). Kupffer cell:hepatocyte cocultures release nitric oxide in response to bacterial endotoxin. J Surg Res, 48, 349-53. 
Billiar, T.R., Curran, R.D., Stuehr, D.J., Ferrari, F.K. \& Simmons, R.L. (1989). Evidence that activation of Kupffer cells results in production of L-arginine metabolites that release cell-associated iron and inhibit hepatocyte protein synthesis. Surgery, 106, 364-71; discussion 371-2.

Black, S.M. \& Ortiz de Montellano, P.R. (1995). Characterization of rat neuronal nitric oxide synthase expressed in Saccharomyces cerevisiae. DNA Cell Biol, 14, 78994.

Blenis, J. \& Resh, M.D. (1993). Subcellular localization specified by protein acylation and phosphorylation. Curr Opin Cell Biol, 5, 984-9.

Block, E.R., Herrera, H. \& Couch, M. (1995). Hypoxia inhibits L-arginine uptake by pulmonary artery endothelial cells. Am J Physiol, 269, pL574-80.

Bogovski, P. \& Bogovski, S. (1981). Animal Species in which N-nitroso compounds induce cancer. Int $J$ Cancer, 27, 471-4.

Bohme, G.A., Bon, C., Lemaire, M., Reibaud, M., Piot, O., Stutzmann, J.M., Doble, A. \& Blanchard, J.C. (1993). Altered synaptic plasticity and memory formation in nitric oxide synthase inhibitor-treated rats. Proc Natl Acad Sci U S A, 90, 9191-4.

Boje, K.M. \& Arora, P.K. (1992). Microglial-produced nitric oxide and reactive nitrogen oxides mediate neuronal cell death. Brain Res, 587, 250-6.

Bolotina, V.M., Najibi, S., Palacino, J.J., Pagano, P.J. \& Cohen, R.A. (1994). Nitric oxide directly activates calcium-dependent potassium channels in vascular smooth muscle. Nature, 368, 850-3.

Boucher, J.L., Genet, A., Vadon, S., Delaforge, M., Henry, Y. \& Mansuy, D. (1992). Cytochrome $\mathrm{P} 450$ catalyzes the oxidation of $\mathrm{N}$ omega-hydroxy-L-arginine by NADPH and $\mathrm{O} 2$ to nitric oxide and citrulline. Biochem Biophys Res Commun, 187, 880-6.

Bredt, D.S. (1998). NO skeletal muscle derived relaxing factor in Duchenne muscular dystrophy [comment]. Proc Natl Acad Sci U S A, 95, 14592-3.

Bredt, D.S., Ferris, C.D. \& Snyder, S.H. (1992). Nitric oxide synthase regulatory sites. Phosphorylation by cyclic AMP-dependent protein kinase, protein kinase $\mathrm{C}$, and calcium/calmodulin protein kinase; identification of flavin and calmodulin binding sites. J Biol Chem, 267, 10976-81.

Bredt, D.S., Hwang, P.M., Glatt, C.E., Lowenstein, C., Reed, R.R. \& Snyder, S.H. (1991). Cloned and expressed nitric oxide synthase structurally resembles cytochrome P-450 reductase. Nature, $351,714-8$.

Bredt, D.S., Hwang, P.M. \& Snyder, S.H. (1990). Localization of nitric oxide synthase indicating a neural role for nitric oxide. Nature, 347, 768-70. 
Bredt, D.S. \& Snyder, S.H. (1990). Isolation of nitric oxide synthetase, a calmodulinrequiring enzyme. Proc Natl Acad Sci U S A, 87, 682-5.

Brenman, J.E., Chao, D.S., Gee, S.H., McGee, A.W., Craven, S.E., Santillano, D.R., Wu, Z., Huang, F., Xia, H., Peters, M.F., Froehner, S.C. \& Bredt, D.S. (1996). Interaction of nitric oxide synthase with the postsynaptic density protein PSD-95 and alphal-syntrophin mediated by PDZ domains. Cell, 84, 757-67.

Brenman, J.E., Chao, D.S., Xia, H., Aldape, K. \& Bredt, D.S. (1995). Nitric oxide synthase complexed with dystrophin and absent from skeletal muscle sarcolemma in Duchenne muscular dystrophy. Cell, 82, 743-52.

Brenman, J.E., Christopherson, K.S., Craven, S.E., McGee, A.W. \& Bredt, D.S. (1996). Cloning and characterization of postsynaptic density 93, a nitric oxide synthase interacting protein. $J$ Neurosci, 16, 7407-15.

Brenman, J.E., Xia, H., Chao, D.S., Black, S.M. \& Bredt, D.S. (1997). Regulation of neuronal nitric oxide synthase through alternative transcripts. Dev Neurosci, 19, 22431.

Brickey, D.A., Bann, J.G., Fong, Y.L., Perrino, L., Brennan, R.G. \& Soderling, T.R. (1994). Mutational analysis of the autoinhibitory domain of calmodulin kinase II. $J$ Biol Chem, 269, 29047-54.

Brown, G.C. (1995). Reversible binding and inhibition of catalase by nitric oxide. Eur J Biochem, 232, 188-91.

Brune, B., Gotz, C., Messmer, U.K., Sandau, K., Hirvonen, M.R. \& Lapetina, E.G. (1997). Superoxide formation and macrophage resistance to nitric oxide-mediated apoptosis. J Biol Chem, 272, 7253-8.

Brune, B. \& Lapetina, E.G. (1991). Phosphorylation of nitric oxide synthase by protein kinase A. Biochem Biophys Res Commun, 181, 921-6.

Brune, B., Mohr, S. \& Messmer, U.K. (1996). Protein thiol modification and apoptotic cell death as cGMP-independent nitric oxide (NO) signaling pathways. Rev Physiol Biochem Pharmacol, 127, 1-30.

Buga, G.M., Griscavage, J.M., Rogers, N.E. \& Ignarro, L.J. (1993). Negative feedback regulation of endothelial cell function by nitric oxide. Circ Res, 73, 808-12.

Burnett, A.L., Lowenstein, C.J., Bredt, D.S., Chang, T.S. \& Snyder, S.H. (1992). Nitric oxide: a physiologic mediator of penile erection. Science, 257, 401-3.

Busconi, L. \& Michel, T. (1993). Endothelial nitric oxide synthase. N-terminal myristoylation determines subcellular localization. J Biol Chem, 268, 8410-3.

Busse, R. \& Fleming, I. (1998). Regulation of NO synthesis in endothelial cells. Kidney Blood Press Res, 21, 264-6. 
Butt, E., Bernhardt, M., Smolenski, A., Kotsonis, P., Frohlich, L.G., Sickmann, A., Meyer, H.E., Lohmann, S.M. \& Schmidt, H.H. (2000). Endothelial nitric-oxide synthase (type III) is activated and becomes calcium independent upon phosphorylation by cyclic nucleotide-dependent protein kinases. J Biol Chem, 275, p5179-87.

Caulin-Glaser, T., Garcia-Cardena, G., Sarrel, P., Sessa, W.C. \& Bender, J.R. (1997). 17 beta-estradiol regulation of human endothelial cell basal nitric oxide release, independent of cytosolic Ca2+ mobilization. Circ Res, 81, 885-92.

Chabin, R.M., McCauley, E., Calaycay, J.R., Kelly, T.M., MacNaul, K.L., Wolfe, G.C., Hutchinson, N.I., Madhusudanaraju, S., Schmidt, J.A., Kozarich, J.W. \& Wong, K.K. (1996). Active-site structure analysis of recombinant human inducible nitric oxide synthase using imidazole. Biochemistry, $35,9567-75$.

Chao, C.C., Hu, S., Molitor, T.W., Shaskan, E.G. \& Peterson, P.K. (1992). Activated microglia mediate neuronal cell injury via a nitric oxide mechanism. J Immunol, 149, 2736-41.

Chao, D.S., Silvagno, F., Xia, H., Cornwell, T.L., Lincoln, T.M. \& Bredt, D.S. (1997). Nitric oxide synthase and cyclic GMP-dependent protein kinase concentrated at the neuromuscular endplate. Neuroscience, $76,665-72$.

Chartrain, N.A., Geller, D.A., Koty, P.P., Sitrin, N.F., Nussler, A.K., Hoffman, E.P., Billiar, T.R., Hutchinson, N.I. \& Mudgett, J.S. (1994). Molecular cloning, structure, and chromosomal localization of the human inducible nitric oxide synthase gene. $J$ Biol Chem, 269, 6765-72.

Chen, J., Zhang, S., Zuo, P. \& Tang, L. (1997). Memory-related changes of nitric oxide synthase activity and nitrite level in rat brain. Neuroreport, 8, 1771-4.

Chen, P.-F. \& Wu, K.K. (2000). Characterization of the roles of the 594-645 region in human endothelial nitric oxide synthase in regulating calmodulin binding and electron transfer. J. Biol. Chem, 275, 13155-13163.

Chen, P.F., Tsai, A.L., Berka, V. \& Wu, K.K. (1996). Endothelial nitric-oxide synthase. Evidence for bidomain structure and successful reconstitution of catalytic activity from two separate domains generated by a baculovirus expression system. $J$ Biol Chem, 271, 14631-5.

Chen, P.F., Tsai, A.L., Berka, V. \& Wu, K.K. (1997). Mutation of Glu-361 in human endothelial nitric-oxide synthase selectively abolishes L-arginine binding without perturbing the behavior of heme and other redox centers. J Biol Chem, 272, 6114-8.

Chen, P.F., Tsai, A.L. \& Wu, K.K. (1994). Cysteine 184 of endothelial nitric oxide synthase is involved in heme coordination and catalytic activity. J Biol Chem, 269, 25062-6. 
Chen, Z.P., Mitchelhill, K.I., Michell, B.J., Stapleton, D., Rodriguez-Crespo, I., Witters, L.A., Power, D.A., Ortiz de Montellano, P.R. \& Kemp, B.E. (1999). AMPactivated protein kinase phosphorylation of endothelial NO synthase. FEBS Lett, 443, 285-9.

Cho, H.J., Martin, E., Xie, Q.W., Sassa, S. \& Nathan, C. (1995). Inducible nitric oxide synthase: identification of amino acid residues essential for dimerization and binding of tetrahydrobiopterin. Proc Natl Acad Sci U S A, 92, 11514-8.

Cho, H.J., Xie, Q.W., Calaycay, J., Mumford, R.A., Swiderek, K.M., Lee, T.D. \& Nathan, C. (1992). Calmodulin is a subunit of nitric oxide synthase from macrophages. J Exp Med, 176, 599-604.

Chu, S.C., Wu, H.P., Banks, T.C., Eissa, N.T. \& Moss, J. (1995). Structural diversity in the 5'-untranslated region of cytokine-stimulated human inducible nitric oxide synthase mRNA. J Biol Chem, 270, 10625-30.

Chun, S.Y., Eisenhauer, K.M., Kubo, M. \& Hsueh, A.J. (1995). Interleukin-1 beta suppresses apoptosis in rat ovarian follicles by increasing nitric oxide production. Endocrinology, 136, 3120-7.

Cieslik, K., Zembowicz, A., Tang, J.L. \& Wu, K.K. (1998). Transcriptional regulation of endothelial nitric-oxide synthase by lysophosphatidylcholine. J Biol Chem, 273, 14885-90.

Clancy, R.M., Levartovsky, D., Leszczynska-Piziak, J., Yegudin, J. \& Abramson, S.B. (1994). Nitric oxide reacts with intracellular glutathione and activates the hexose monophosphate shunt in human neutrophils: evidence for S-nitrosoglutathione as a bioactive intermediary. Proc Natl Acad Sci U S A, 91, 3680-4.

Coffer, P.J., Jin, J. \& Woodgett, J.R. (1998). Protein kinase B (c-Akt): a multifunctional mediator of phosphatidylinositol 3-kinase activation. Biochem J, 335, $\mathrm{p} 1-13$.

Cohen, J.J. (1993). Apoptosis. Immunol Today, 14, 126-30.

Cohen, P., Burchell, A., Foulkes, J.G. \& Cohen, P.T. (1978). Identification of the $\mathrm{Ca} 2+$-dependent modulator protein as the fourth subunit of rabbit skeletal muscle phosphorylase kinase. FEBS Lett, 92, 287-93.

Colasanti, M., Persichini, T., Menegazzi, M., Mariotto, S., Giordano, E., Caldarera, C.M., Sogos, V., Lauro, G.M. \& Suzuki, H. (1995). Induction of nitric oxide synthase mRNA expression. Suppression by exogenous nitric oxide. J Biol Chem, 270, 267313.

Cooke, J.P., Dzau, J. \& Creager, A. (1991). Endothelial dysfunction in hypercholesterolemia is corrected by L-arginine. Basic Res Cardiol, 2, 173-81.

Corbett, J.A., Kwon, G., Turk, J. \& McDaniel, M.L. (1993). IL-1 beta induces the coexpression of both nitric oxide synthase and cyclooxygenase by islets of 
Langerhans: activation of cyclooxygenase by nitric oxide. Biochemistry, 32, 1376770.

Craven, P.A. \& De Rubertis, F.R. (1978). Restoration of the responsiveness of purified guanylate cyclase to nitrosoguanidine, nitric oxide, and related activators by heme and hemeproteins. Evidence for involvement of the paramagnetic nitrosyl-heme complex in enzyme activation. J Biol Chem, 253, 8433-43.

Cross, A.H., Manning, P.T., Stern, M.K. \& Misko, T.P. (1997). Evidence for the production of peroxynitrite in inflammatory CNS demyelination. $J$ Neuroimmunol, $80,121-30$.

Curran, R.D., Billiar, T.R., Stuehr, D.J., Hofmann, K. \& Simmons, R.L. (1989). Hepatocytes produce nitrogen oxides from $\mathrm{L}$-arginine in response to inflammatory products of Kupffer cells. J Exp Med, 170, 1769-74.

Daff, S., Sagami, S. \& Shimizu, T. (1999). The 42-amino acid insert in the FMN domain of neuronal nitric oxide synthase exerts control over $\mathrm{Ca}^{2}+/$ Calmodulindependent electron transfer. J. Biol. Chem., 274, 30589-30595.

Dayhoff, M.O., Hunt, W.C. \& Hunt, L.T. (1983). Meth Enzymol, 91, 524-545.

Deckers-Hebestreit, G., Schmid, R., Kiltz, H.H. \& Altendorf, K. (1987). F0 portion of Escherichia coli ATP synthase: orientation of subunit $c$ in the membrane. Biochemistry, 26, 5486-92.

Deguchi, T. \& Yoshioka, M. (1982). L-Arginine identified as an endogenous activator for soluble guanylate cyclase from neuroblastoma cells. J Biol Chem, 257, 10147-51.

dela Torre, A., Schroeder, R.A., Bartlett, S.T. \& Kuo, P.C. (1998). Differential effects of nitric oxide-mediated S-nitrosylation on p50 and c-jun DNA binding. Surgery, 124, $137-142$.

Deng, A.Y., Rapp, J.P., Kato, H. \& Bihoreau, M.T. (1995). Linkage mapping of the neuronal nitric oxide synthase gene (Nos1) to rat chromosome 12. Mamm Genome, 6, 824.

Dimmeler, S., Assmus, B., Hermann, C., Haendeler, J. \& Zeiher, A.M. (1998). Fluid shear stress stimulates phosphorylation of Akt in human endothelial cells: involvement in suppression of apoptosis. Circ Res, 83, 334-41.

Dimmeler, S. \& Brune, B. (1992). Characterization of a nitric-oxide-catalysed ADPribosylation of glyceraldehyde-3-phosphate dehydrogenase. Eur J Biochem, 210, 30510.

Dimmeler, S., Dernbach, E. \& Zeiher, A.M. (2000). Phosphorylation of the endothelial nitric oxide synthase at ser-1177 is required for VEGF-induced endothelial cell migration. FEBS Lett, 477, 258-262. 
Dimmeler, S., Fleming, I., Fisslthaler, B., Hermann, C., Busse, R. \& Zeiher, A.M. (1999). Activation of nitric oxide synthase in endothelial cells by Akt-dependent phosphorylation. Nature, 399, 601-5.

Dimmeler, S., Haendeler, J., Nehls, M. \& Zeiher, A.M. (1997). Suppression of apoptosis by nitric oxide via inhibition of interleukin-1beta-converting enzyme (ICE)like and cysteine protease protein (CPP)-32-like proteases. J Exp Med, 185, 601-7.

Dimmeler, S., Haendeler, J., Sause, A. \& Zeiher, A.M. (1998). Nitric oxide inhibits APO-1/Fas-mediated cell death. Cell Growth Differ, 9, 415-22.

Dimmeler, S. \& Zeiher, A.M. (1997). Nitrix oxide and Apoptosis: another paradigm for the double edged role of nitric oxide. Nitric oxide: Biology and Chemistry, 1, 275281.

Dinerman, J.L., Steiner, J.P., Dawson, T.M., Dawson, V. \& Snyder, S.H. (1994). Cyclic nucleotide dependent phosphorylation of neuronal nitric oxide synthase inhibits catalytic activity. Neuropharmacology, 33, 1245-51.

Dix, T.A., Kuhn, D.M. \& Benkovic, S.J. (1987). Mechanism of oxygen activation by tyrosine hydroxylase. Biochemistry, 26, 3354-61.

Downward, J. (1998). Mechanisms and consequences of activation of protein kinase B/Akt. Curr Opin Cell Biol, 10, p262-7.

Drapier, J.C. \& Hibbs, J.B., Jr. (1988). Differentiation of murine macrophages to express nonspecific cytotoxicity for tumor cells results in L-arginine-dependent inhibition of mitochondrial iron-sulfur enzymes in the macrophage effector cells. $J$ Immunol, 140, 2829-38.

Drapier, J.C., Hirling, H., Wietzerbin, J., Kaldy, P. \& Kuhn, L.C. (1993). Biosynthesis of nitric oxide activates iron regulatory factor in macrophages. Embo J, 12, 3643-9.

Duhe, R.J., Nielsen, M.D., Dittman, A.H., Villacres, E.C., Choi, E.J. \& Storm, D.R. (1994). Oxidation of critical cysteine residues of type I adenylyl cyclase by oiodosobenzoate or nitric oxide reversibly inhibits stimulation by calcium and calmodulin. J Biol Chem, 269, 7290-6.

Eigler, A., Moeller, J. \& Endres, S. (1995). Exogenous and endogenous nitric oxide attenuates tumor necrosis factor synthesis in the murine macrophage cell line RAW 264.7. J Immunol, 154, 4048-54.

Ellis, R.E., Yuan, J.Y. \& Horvitz, H.R. (1991). Mechanisms and functions of cell death. Annu Rev Cell Biol, 7, 663-98.

Enari, M., Talanian, R.V., Wong, W.W. \& Nagata, S. (1996). Sequential activation of ICE-like and CPP32-like proteases during Fas-mediated apoptosis. Nature, 380, 723 6. 
Estevez, A.G., Radi, R., Barbeito, L., Shin, J.T., Thompson, J.A. \& Beckman, J.S. (1995). Peroxynitrite-induced cytotoxicity in PC12 cells: evidence for an apoptotic mechanism differentially modulated by neurotrophic factors. $J$ Neurochem, 65,1543 . 50.

Fabiato, A. \& Fabiato, F. (1979). Calculator programs for computing the composition of the solutions containing multiple metals and ligands used for experiments in skinned muscle cells. J Physiol (Paris), 75, 463-505.

Fehsel, K., Kroncke, K.D., Meyer, K.L., Huber, H., Wahn, V. \& Kolb-Bachofen, V. (1995). Nitric oxide induces apoptosis in mouse thymocytes. J Immunol, 155, 285865.

Feldman, P.L., Griffith, O.W., Hong, H. \& Stuehr, D.J. (1993). Irreversible inactivation of macrophage and brain nitric oxide synthase by L-NG-methylarginine requires NADPH-dependent hydroxylation. J Med Chem, 36, 491-6.

Feron, O., Belhassen, L., Kobzik, L., Smith, T.W., Kelly, R.A. \& Michel, T. (1996). Endothelial nitric oxide synthase targeting to caveolae. Specific interactions with caveolin isoforms in cardiac myocytes and endothelial cells. $J$ Biol Chem, 271, $22810-4$.

Feron, O., Dessy, C., Opel, D.J., Arstall, M.A., Kelly, R.A. \& Michel, T. (1998). Modulation of the endothelial nitric-oxide synthase-caveolin interaction in cardiac myocytes. Implications for the autonomic regulation of heart rate. J Biol Chem, 273, 30249-54.

Feron, O., Michel, J.B., Sase, K. \& Michel, T. (1998). Dynamic regulation of endothelial nitric oxide synthase: complementary roles of dual acylation and caveolin interactions. Biochemistry, 37, 193-200.

Fisslthaler, B., Dimmeler, S., Hermann, C., Busse, R. \& Fleming, I. (2000). Phosphorylation and activation of the endothelial nitric oxide synthase by fluid shear stress. Acta Physiol Scand, 168, p81-8.

Fleming, I., Bauersachs, J. \& Busse, R. (1997). Calcium-dependent and calciumindependent activation of the endothelial NO synthase. J Vasc Res, 34, 165-74.

Fleming, I., Fisslthaler, B. \& Busse, R. (1996). Interdependence of calcium signaling and protein tyrosine phosphorylation in human endothelial cells. $J$ Biol Chem, 271, 11009-15.

Fleming, I., Hecker, M. \& Busse, R. (1994). Intracellular alkalinization induced by bradykinin sustains activation of the constitutive nitric oxide synthase in endothelial cells. Circ Res, 74, 1220-6.

Forstermann, U., Gath, I., Schwarz, P., Closs, E.I. \& Kleinert, H. (1995). Isoforms of nitric oxide synthase. Properties, cellular distribution and expressional control. Biochem Pharmacol, 50, 1321-32. 
Forstermann, U., Mulsch, A., Bohme, E. \& Busse, R. (1986). Stimulation of soluble guanylate cyclase by an acetylcholine-induced endothelium-derived factor from rabbit and canine arteries. Circ Res, 58, 531-8.

Fossetta, J.D., Niu, X.D., Lunn, C.A., Zavodny, P.J., Narula, S.K. \& Lundell, D. (1996). Expression of human inducible nitric oxide synthase in Escherichia coli. FEBS Lett, 379, 135-8.

Fujisawa, H., Ogura, T., Kurashima, Y., Yokoyama, T., Yamashita, J. \& Esumi, H. (1994). Expression of two types of nitric oxide synthase mRNA in human neuroblastoma cell lines. J Neurochem, 63, 140-5.

Fukuto, J.M., Chiang, K., Hszieh, R., Wong, P. \& Chaudhuri, G. (1992). The pharmacological activity of nitroxyl: a potent vasodilator with activity similar to nitric oxide and/or endothelium-derived relaxing factor. J Pharmacol Exp Ther, 263, 54651.

Fukuto, J.M., Hobbs, A.J. \& Ignarro, L.J. (1993). Conversion of nitroxyl (HNO) to nitric oxide (NO) in biological systems: the role of physiological oxidants and relevance to the biological activity of HNO. Biochem Biophys Res Commun, 196, 707-13.

Fukuto, J.M., Stuehr, D.J., Feldman, P.L., Bova, M.P. \& Wong, P. (1993). Peracid oxidation of an $\mathrm{N}$-hydroxyguanidine compound: a chemical model for the oxidation of $\mathrm{N}$ omega-hydroxyl-L-arginine by nitric oxide synthase. J Med Chem, 36, 2666-70.

Fulton, D., Gratton, J.P., McCabe, T.J., Fontana, J., Fujio, Y., Walsh, K., Franke, T.F., Papapetropoulos, A. \& Sessa, W.C. (1999). Regulation of endothelium-derived nitric oxide production by the protein kinase Akt. Nature, 399, 597-601.

Furchgott, R.F. (1988). The basis for the proposal that the acid-activatable inhibitory factor from bovine retractor penis is inorganic nitrite and the endothelium-derived relaxing factor is nitric oxide. In Vasodilatation: Vascular Smooth Muscle, Peptides, Autonomic Nerves and Endothelium. ed. Vanhoutte, P.M. pp. 400-414. New York: Raven Press Ltd.

Furchgott, R.F. (1988). Studies on relaxation of rabbit aorta by sodium nitrite: the basis for the proposal that the acid-activatable inhibitory factor from bovine retractor penis is inorganic nitrite and the endothelium derived relaxing factor is nitric oxide. In Vasodilation: Vascular smooth muscle, Peptides, Autonomic nerves, and Endothelium. ed. Vanhoutte, P.M. pp. 401-414. New York: Raven.

Furchgott, R.F. \& Zawadzki, J.V. (1980). The obligatory role of endothelial cells in the relaxation of arterial smooth muscle by acetylcholine. Nature, 288, 373-6.

Gachhui, R., Presta, A., Bentley, D.F., Abu-Soud, H.M., McArthur, R., Brudvig, G., Ghosh, D.K. \& Stuehr, D.J. (1996). Characterization of the reductase domain of rat neuronal nitric oxide synthase generated in the methylotrophic yeast Pichia pastoris. Calmodulin response is complete within the reductase domain itself. $J$ Biol Chem, 271, 20594-602. 
Gallis, B., Corthals, G.L., Goodlett, D.R., Ueba, H., Kim, F., Presnell, S.R., Figeys, D., Harrison, D.G., Berk, B.C., Aebersold, R. \& Corson, M.A. (1999). Identification of flow-dependent endothelial nitric-oxide synthase phosphorylation sites by mass spectrometry and regulation of phosphorylation and nitric oxide production by the phosphatidylinositol 3-kinase inhibitor LY294002. J Biol Chem, 274, 30101-8.

Garcia-Cardena, G., Fan, R., Shah, V., Sorrentino, R., Cirino, G., Papapetropoulos, A. \& Sessa, W.C. (1998). Dynamic activation of endothelial nitric oxide synthase by Hsp90. Nature, 392, 821-4.

Garcia-Cardena, G., Fan, R., Stern, D.F., Liu, J. \& Sessa, W.C. (1996). Endothelial nitric oxide synthase is regulated by tyrosine phosphorylation and interacts with caveolin-1. J Biol Chem, 271, 27237-40.

Garcia-Cardena, G., Martasek, P., Masters, B.S., Skidd, P.M., Couet, J., Li, S., Lisanti, M.P. \& Sessa, W.C. (1997). Dissecting the interaction between nitric oxide synthase (NOS) and caveolin. Functional significance of the nos caveolin binding domain in vivo. $J$ Biol Chem, 272, 25437-40.

Garcia-Cardena, G., Oh, P., Liu, J., Schnitzer, J.E. \& Sessa, W.C. (1996). Targeting of nitric oxide synthase to endothelial cell caveolae via palmitoylation: implications for nitric oxide signaling. Proc Natl Acad Sci U S A, 93, 6448-53.

Garthwaite, J., Charles, S.L. \& Chess-Williams, R. (1988). Endothelium-derived relaxing factor release on activation of NMDA receptors suggests role as intercellular messenger in the brain. Nature, 336, 385-8.

Garvey, E.P., Tuttle, J.V., Covington, K., Merrill, B.M., Wood, E.R., Baylis, S.A. \& Charles, I.G. (1994). Purification and characterization of the constitutive nitric oxide synthase from human placenta. Arch Biochem Biophys, 311, 235-41.

Gaston, B., Drazen, J.M., Jansen, A., Sugarbaker, D.A., Loscalzo, J., Richards, W. \& Stamler, J.S. (1994). Relaxation of human bronchial smooth muscle by S-nitrosothiols in vitro. J Pharmacol Exp Ther, 268, 978-84.

Geller, D.A., Lowenstein, C.J., Shapiro, R.A., Nussler, A.K., Di Silvio, M., Wang, S.C., Nakayama, D.K., Simmons, R.L., Snyder, S.H. \& Billiar, T.R. (1993). Molecular cloning and expression of inducible nitric oxide synthase from human hepatocytes. Proc Natl Acad Sci U S A, 90, 3491-5.

Genaro, A.M., Hortelano, S., Alvarez, A., Martinez, C. \& Bosca, L. (1995). Splenic B lymphocyte programmed cell death is prevented by nitric oxide release through mechanisms involving sustained Bcl-2 levels. J Clin Invest, 95, 1884-90.

Gerber, N.C., Nishida, C.R. \& Ortiz de Montellano, P.R. (1997). Characterization of human liver inducible nitric oxide synthase expressed in Escherichia coli. Arch Biochem Biophys, 343, 249-53.

Gerber, N.C. \& Ortiz de Montellano, P.R. (1995). Neuronal nitric oxide synthase. Expression in Escherichia coli, irreversible inhibition by phenyldiazene, and active site topology. J Biol Chem, 270, 17791-6. 
Gergel, D. \& Cederbaum, A.I. (1996). Inhibition of the catalytic activity of alcohol dehydrogenase by nitric oxide is associated with $S$ nitrosylation and the release of zinc. Biochemistry, 35, 16186-94.

Ghosh, D.K., Abu-Soud, H.M. \& Stuehr, D.J. (1996). Domains of macrophage N(O) synthase have divergent roles in forming and stabilizing the active dimeric enzyme. Biochemistry, 35, 1444-9.

Ghosh, D.K. \& Stuehr, D.J. (1995). Macrophage NO synthase: characterization of isolated oxygenase and reductase domains reveals a head-to-head subunit interaction. Biochemistry, 34, 801-7.

Ghosh, S., Gachhui, R., Crooks, C., Wu, C., Lisanti, M.P. \& Stuehr, D.J. (1998). Interaction between caveolin-1 and the reductase domain of endothelial nitric-oxide synthase. Consequences for catalysis. J Biol Chem, 273, 22267-71.

Gilbert, R.S. \& Herschman, H.R. (1993). Transforming growth factor beta differentially modulates the inducible nitric oxide synthase gene in distinct cell types. Biochem Biophys Res Commun, 195, 380-4.

Gillardon, F., Krep, H., Brinker, G., Lenz, C., Bottiger, B. \& Hossmann, K.A. (1998). Induction of protein inhibitor of neuronal nitric oxide synthase/cytoplasmic dynein light chain following cerebral ischemia. Neuroscience, 84, 81-8.

Giovanelli, J., Campos, K.L. \& Kaufman, S. (1991). Tetrahydrobiopterin, a cofactor for rat cerebellar nitric oxide synthase, does not function as a reactant in the oxygenation of arginine. Proc Natl Acad Sci U S A, 88, 7091-5.

Goldring, C.E., Narayanan, R., Lagadec, P. \& Jeannin, J.F. (1995). Transcriptional inhibition of the inducible nitric oxide synthase gene by competitive binding of NFkappa B/Rel proteins. Biochem Biophys Res Commun, 209, 73-9.

Golser, R., Gorren, A.C., Leber, A., Andrew, P., Habisch, H.J., Werner, E.R., Schmidt, K., Venema, R.C. \& Mayer, B. (2000). Interaction of endothelial and neuronal nitric-oxide synthases with the bradykinin $\mathrm{B} 2$ receptor. Binding of an inhibitory peptide to the oxygenase domain blocks uncoupled NADPH oxidation. $J$ Biol Chem, 275, p5291-6.

Gopalakrishna, R., Chen, Z.H. \& Gundimeda, U. (1993). Nitric oxide and nitric oxide-generating agents induce a reversible inactivation of protein kinase $\mathrm{C}$ activity and phorbol ester binding. J Biol Chem, 268, 27180-5.

Grand, R.J., Shenolikar, S. \& Cohen, P. (1981). The amino acid sequence of the delta subunit (calmodulin) of rabbit skeletal muscle phosphorylase kinase. Eur J Biochem, $113,359-67$.

Granger, D.L. \& Lehninger, A.L. (1982). Sites of inhibition of mitochondrial electron transport in macrophage-injured neoplastic cells. J Cell Biol, 95, 527-35. 
Granger, D.L., Taintor, R.R., Cook, J.L. \& Hibbs, J.B., Jr. (1980). Injury of neoplastic cells by murine macrophages leads to inhibition of mitochondrial respiration. $J$ Clin Invest, 65, 357-70.

Gratton, J.P., Fontana, J., O'Connor, D.S., Garcia-Cardena, G., McCabe, T.J. \& Sessa, W.C. (2000). Reconstitution of an endothelial nitric oxide synthase (eNOS), hsp90, and caveolin-1 complex in vitro. Evidence that hsp90 facilitates calmodulin stimulated displacement of eNOS from caveolin-1. J. Biol. Chem., 275, 22268.

Green, L.C., Ruiz de Luzuriaga, K., Wagner, D.A., Rand, W., Istfan, N., Young, V.R. \& Tannenbaum, S.R. (1981). Nitrate biosynthesis in man. Proc Natl Acad Sci US A, 78, 7764-8.

Green, L.C., Tannenbaum, S.R. \& Goldman, P. (1981). Nitrate synthesis in the germfree and conventional rat. Science, 212, 56-8.

Green, S.J., Mellouk, S., Hoffman, S.L., Meltzer, M.S. \& Nacy, C.A. (1990). Cellular mechanisms of nonspecific immunity to intracellular infection: cytokine-induced synthesis of toxic nitrogen oxides from L-arginine by macrophages and hepatocytes. Immunol Lett, 25, 15-9.

Greenwood, M.T., Guo, Y., Kumar, U., Beausejours, S. \& Hussain, S.N. (1997). Distribution of protein inhibitor of neuronal nitric oxide synthase in rat brain. Biochem Biophys Res Commun, 238, 617-21.

Griffith, O.W. \& Stuehr, D.J. (1995). Nitric oxide synthases: properties and catalytic mechanism. Annu Rev Physiol, 57, 707-36.

Grilli, M., Chiu, J.J. \& Lenardo, M.J. (1993). NF-kappa B and Rel: participants in a multiform transcriptional regulatory system. Int Rev Cytol, 143, 1-62.

Griscavage, J.M., Rogers, N.E., Sherman, M.P. \& Ignarro, L.J. (1993). Inducible nitric oxide synthase from a rat alveolar macrophage cell line is inhibited by nitric oxide. J Immunol, 151, 6329-37.

Gross, S.S. (1996). Microtiter plate assay for determining kinetics of nitric oxide synthesis. Methods Enzymol, 268, 159-68.

Gross, S.S., Jaffe, E.A., Levi, R. \& Kilbourn, R.G. (1991). Cytokine-activated endothelial cells express an isotype of nitric oxide synthase which is tetrahydrobiopterin-dependent, calmodulin-independent and inhibited by arginine analogs with a rank-order of potency characteristic of activated macrophages. Biochem Biophys Res Commun, 178, 823-9.

Gross, S.S. and Wolin,W.M.S. (1995). Nitric oxide: pathopysiological mechanisms. Annu. Rev. Physiol., 57, 737-769.

Gruetter, C.A., Barry, B.K., McNamara, D.B., Gruetter, D.Y., Kadowitz, P.J. \& Ignarro, L. (1979). Relaxation of bovine coronary artery and activation of coronary 
arterial guanylate cyclase by nitric oxide, nitroprusside and a carcinogenic nitrosoamine. J Cyclic Nucleotide Res, 5, 211-24.

Guo, F.H., De Raeve, H.R., Rice, T.W., Stuehr, D.J., Thunnissen, F.B. \& Erzurum, S.C. (1995). Continuous nitric oxide synthesis by inducible nitric oxide synthase in normal human airway epithelium in vivo. Proc Natl Acad Sci U S A, 92, 7809-13.

Gutteridge, J.M. (1989). Iron and oxygen: a biologically damaging mixture. Acta Paediatr Scand Suppl, 361, 78-85.

Hall, A.V., Antoniou, H., Wang, Y., Cheung, A.H., Arbus, A.M., Olson, S.L., Lu, W.C., Kau, C.L. \& Marsden, P.A. (1994). Structural organization of the human neuronal nitric oxide synthase gene (NOS1). J Biol Chem, 269, 33082-90.

Harbrecht, B.G., Wang, S.C., Simmons, R.L. \& Billiar, T.R. (1995). Cyclic GMP and guanylate cyclase mediate lipopolysaccharide-induced Kupffer cell tumor necrosis factor-alpha synthesis. J Leukoc Biol, 57, 297-302.

Harteneck, C., Klatt, P., Schmidt, K. \& Mayer, B. (1994). Expression of rat brain nitric oxide synthase in baculovirus-infected insect cells and characterization of the purified enzyme. Biochem $J, 304,683-6$.

Hayashi, S., Chan, C.C., Gazzinelli, R. \& Roberge, F.G. (1996). Contribution of nitric oxide to the host parasite equilibrium in toxoplasmosis. J Immunol, 156, 1476-81.

Hecker, M., Sessa, W.C., Harris, H.J., Anggard, E.E. \& Vane, J.R. (1990). The metabolism of L-arginine and its significance for the biosynthesis of endotheliumderived relaxing factor: cultured endothelial cells recycle L-citrulline to L-arginine. Proc Natl Acad Sci U S A, 87, 8612-6.

Heinzel, B., John, M., Klatt, P., Bohme, E. \& Mayer, B. (1992). Ca2+/calmodulindependent formation of hydrogen peroxide by brain nitric oxide synthase. Biochem $J$, 281, 627-30.

Hellstrom, W.J., Monga, M., Wang, R., Domer, F.R., Kadowitz, P.J. \& Roberts, J.A. (1994). Penile erection in the primate: induction with nitric-oxide donors. J Urol, 151, 1723-7.

Hendriks, W. (1995). Neuronal nitric oxide synthase contains a discs-large homologous region (DHR) sequence motif [letter]. Biochem J, 305, 687-8.

Hevel, J.M. \& Marletta, M.A. (1992). Macrophage nitric oxide synthase: relationship between enzyme-bound tetrahydrobiopterin and synthase activity. Biochemistry, 31, 7160-5.

Hevel, J.M., White, K.A. \& Marletta, M.A. (1991). Purification of the inducible murine macrophage nitric oxide synthase: Identification as a flavoprotein. $J$ Biol Chem, 266, 22789-91. 
Hibbs, J.B., Jr., Taintor, R.R. \& Vavrin, Z. (1987). Macrophage cytotoxicity: role for L-arginine deiminase and imino nitrogen oxidation to nitrite. Science, 235, 473-6.

Hibbs, J.B., Taintor, R.R., Vavrin, Z., Granger, D.L., Drapier, J.-C. \& al., e. (1990). Synthesis of nitric oxide from a terminal guanido nitrogen atom of L-arginine: a molecular mechanism regulating cellular proliferation that targets intracellular iron. In Nitric oxide from L-arginine. ed. Moncada, S. \& Hibbs, J.B. pp. 189-223. Amsterdam: Elsevier Sci.

Hibbs, J.B., Jr., Taintor, R.R., Vavrin, Z. \& Rachlin, E.M. (1988). Nitric oxide: a cytotoxic activated macrophage effector molecule [published erratum appears in Biochem Biophys Res Commun 1989 Jan 31; 158(2):624]. Biochem Biophys Res Commun, 157, 87-94.

Hibbs, J.B., Jr., Vavrin, Z. \& Taintor, R.R. (1987). L-arginine is required for expression of the activated macrophage effector mechanism causing selective metabolic inhibition in target cells. J Immunol, 138, 550-65.

Hogg, N., Joseph, J. \& Kalyanaraman, B. (1994). The oxidation of alpha-tocopherol and trolox by peroxynitrite. Arch Biochem Biophys, 314, 153-8.

Holscher, C. \& Rose, S.P. (1992). An inhibitor of nitric oxide synthesis prevents memory formation in the chick. Neurosci Lett, 145, $165-7$.

Holzmann, S. (1982). Endothelium-induced relaxation by acetylcholine associated with larger rises in cyclic GMP in coronary arterial strips. J Cyclic Nucleotide Res, 8 , 409-19.

Hou, Y., Guo, Z., Li, J. \& Wang, P.G. (1996). Seleno compounds and glutathione peroxidase catalyzed decomposition of S-nitrosothiols. Biochem Biophys Res Commun, 228, 88-93.

Huang, P.L., Dawson, T.M., Bredt, D.S., Snyder, S.H. \& Fishman, M.C. (1993). Targeted disruption of the neuronal nitric oxide synthase gene. Cell, 75, 1273-86.

Ignarro, L.J., Byrns, R.E. and Woods, K.E. (1988). Biochemical and pharmacological properties of endothelium-derived relaxing factor and its similarity to nitric oxide radical. In Vasodilatation: Vascular Smooth Muscle, Peptides, Autonomic Nerves and Endothelium. ed. Vanhoutte, P.M. pp. 427-436. New York: Raven Press Ltd.

Ignarro, L.J. (1989). Endothelium-derived nitric oxide: actions and properties. Faseb $J, 3,31-6$.

Ignarro, L.J., Buga, G.M., Wood, K.S., Byrns, R.E. \& Chaudhuri, G. (1987). Endothelium-derived relaxing factor produced and released from artery and vein is nitric oxide. Proc Natl Acad Sci U S A, 84, 9265-9. 
Imparl, J.M., Senshu, T. \& Graves, D.J. (1995). Studies of calcineurin-calmodulin interaction: probing the role of arginine residues using peptidylarginine deiminase. Arch Biochem Biophys, 318, 370-377.

Inoue, N., Venema, R.C., Sayegh, H.S., Ohara, Y., Murphy, T.J. \& Harrison, D.G. (1995). Molecular regulation of the bovine endothelial cell nitric oxide synthase by transforming growth factor-beta 1. Arterioscler Thromb Vasc Biol, 15, 1255-61.

Inoue, S. \& Kawanishi, S. (1995). Oxidative DNA damage induced by simultaneous generation of nitric oxide and superoxide. FEBS Lett, 371, 86-8.

Ischiropoulos, H., Zhu, L., Chen, J., Tsai, M., Martin, J.C., Smith, C.D. \& Beckman, J.S. (1992). Peroxynitrite-mediated tyrosine nitration catalyzed by superoxide dismutase. Arch Biochem Biophys, 298, 431-7.

Iwashina, M., Shikiri, M., Marumo, F. \& Hirata, Y. (1998). Transfection of the inducible nitric oxide synthase gene causes apoptosis in vascular smooth muscle cells. Circulation research, $98,1212-1218$.

Iyengar, R., Stuehr, D.J. \& Marletta, M.A. (1987). Macrophage synthesis of nitrite, nitrate, and N-nitrosamines: precursors and role of the respiratory burst. Proc Natl Acad Sci U S A, 84, 6369-73.

Jaenicke, R. (1996). How do proteins acquire their three-dimensional structure and stability? Naturwissenschaften, 83, 544-54.

Jaffrey, S.R., Snowman, A.M., Eliasson, M.J., Cohen, N.A. \& Snyder, S.H. (1998). CAPON: a protein associated with neuronal nitric oxide synthase that regulates its interactions with PSD95. Neuron, 20, 115-24.

Jaffrey, S.R. \& Snyder, S.H. (1996). PIN: an associated protein inhibitor of neuronal nitric oxide synthase. Science, $274,774-7$.

Janssens, S.P., Simouchi, A., Quertermous, T., Bloch, D.B. \& Bloch, K.D. (1992). Cloning and expression of a cDNA encoding human endothelium-derived relating factor/nitric oxide synthase. J Biol Chem, 267, 22694.

Jarrett, H.W. \& Madhavan, R. (1991). Calmodulin-binding proteins also have a calmodulin-like binding site within their structure. The flip-flop model. J Biol Chem, 266, 362-71.

Jenkins, N.A., Rothe, H., Gilbert, D.J., Copeland, N.G. \& Kolb, H. (1994). Mapping of the gene for inducible nitric oxide (NO) synthase of mouse macrophages to chromosome 11, close to Evi-2, nu, and Idd-4. Genomics, 19, 402-4.

Johnson, J.D., Snyder, C., Walsh, M. \& Flynn, M. (1996). Effects of myosin light chain kinase and peptides on $\mathrm{Ca} 2+$ exchange with the $\mathrm{N}$ - and $\mathrm{C}$-terminal $\mathrm{Ca} 2+$ binding sites of calmodulin. J Biol Chem, 271, 761-7. 
Ju, H., Venema, V.J., Marrero, M.B. \& Venema, R.C. (1998). Inhibitory interactions of the bradykinin B2 receptor with endothelial nitric-oxide synthase. J Biol Chem, 273, 24025-9.

Ju, H., Zou, R., Venema, V.J. \& Venema, R.C. (1997). Direct interaction of endothelial nitric-oxide synthase and caveolin-1 inhibits synthase activity. $J$ Biol Chem, 272, 18522-5.

Jung, H.C., Mun, K.H., Park, T.C., Lee, Y.C., Park, J.M., Huh, K., Seong, D.H. \& Suh, J.K. (1997). Role of nitric oxide in penile erection. Yonsei Med J, 38, 261-9.

Kadonaga, J.T., Carner, K.R., Masiarz, F.R. \& Tjian, R. (1987). Isolation of cDNA encoding transcription factor Sp1 and functional analysis of the DNA binding domain. Cell, 51, 1079-90.

Kamijo, R., Harada, H., Matsuyama, T., Bosland, M., Gerecitano, J., Shapiro, D., Le, J., Koh, S.I., Kimura, T., Green, S.J. \& et al. (1994). Requirement for transcription factor IRF-1 in NO synthase induction in macrophages. Science, 263, 1612-5.

Kaneto, H., Fujii, J., Seo, H.G., Suzuki, K., Matsuoka, T., Nakamura, M., Tatsumi, H., Yamasaki, Y., Kamada, T. \& Taniguchi, N. (1995). Apoptotic cell death triggered by nitric oxide in pancreatic beta-cells. Diabetes, $44,733-8$.

Kanner, J., Harel, S. \& Granit, R. (1992). Nitric oxide, an inhibitor of lipid oxidation by lipoxygenase, cyclooxygenase and hemoglobin. Lipids, 27, 46-9.

Katoh, M., Egashira, K., Usui, M., Ichiki, T., Tomita, H., Shimokawa, H., Rakugi, H. \& Takeshita, A. (1998). Cardiac angiotensin II receptors are upregulated by long-term inhibition of nitric oxide synthesis in rats. Circulation Research, 83, 743-751.

Kendrick, K.M., Guevara-Guzman, R., Zorrilla, J., Hinton, M.R., Broad, K.D., Mimmack, M. \& Ohkura, S. (1997). Formation of olfactory memories mediated by nitric oxide. Nature, $388,670-4$.

Kennedy, M.C., Gan, T., Antholine, W.E. \& Petering, D.H. (1993). Metallothionein reacts with $\mathrm{Fe} 2+$ and $\mathrm{NO}$ to form products with $\mathrm{A} \mathrm{g}=2.039 \mathrm{ESR}$ signal. Biochem Biophys Res Commun, 196, 632-5.

Khatsenko, O.G., Gross, S.S., Rifkind, A.B. \& Vane, J.R. (1993). Nitric oxide is a mediator of the decrease in cytochrome P450-dependent metabolism caused by immunostimulants. Proc Natl Acad Sci U S A, 90, 11147-51.

Kilbourn, R.G. \& Belloni, P. (1990). Endothelial cell production of nitrogen oxides in response to interferon gamma in combination with tumor necrosis factor, interleukin1 , or endotoxin. J Natl Cancer Inst, 82, 772-6.

Kilbourn, R.G., Gross, S.S., Jubran, A., Adams, J., Griffith, O.W., Levi, R. \& Lodato, R.F. (1990). NG-methyl-L-arginine inhibits tumor necrosis factor-induced 
hypotension: implications for the involvement of nitric oxide. Proc Natl Acad Sci US A, 87, 3629-32.

Kilbourn, R.G., Gross, S.S., Lodato, R.F., Adams, J., Levi, R., Miller, L.L., Lachman, L.B. \& Griffith, O.W. (1992). Inhibition of interleukin-1-alpha-induced nitric oxide synthase in vascular smooth muscle and full reversal of interleukin-1-alpha-induced hypotension by $\mathrm{N}$ omega-amino-L-arginine. J Natl Cancer Inst, 84, 1008-16.

Kilbourn, R.G., Owen-Schaub, L.B., Cromeens, D.M., Gross, S.S., Flaherty, M.J., Santee, S.M., Alak, A.M. \& Griffith, O.W. (1994). NG-methyl-L-arginine, an inhibitor of nitric oxide formation, reverses IL-2-mediated hypotension in dogs. $J$ Appl Physiol, 76, 1130-7.

Kim, Y.M., de Vera, M.E., Watkins, S.C. \& Billiar, T.R. (1997). Nitric oxide protects cultured rat hepatocytes from tumor necrosis factor-alpha-induced apoptosis by inducing heat shock protein 70 expression. J Biol Chem, 272, 1402-11.

Kishimoto, J., Spurr, N., Liao, M., Lizhi, L., Emson, P. \& Xu, W. (1992). Localization of brain nitric oxide synthase (NOS) to human chromosome 12 [published erratum appears in Genomics $1993 \mathrm{Feb} ; 15(2): 465]$. Genomics, 14, 802-4.

Klatt, P., Heinzel, B., John, M., Kastner, M., Bohme, E. \& Mayer, B. (1992). $\mathrm{Ca} 2+/$ calmodulin-dependent cytochrome $\mathrm{c}$ reductase activity of brain nitric oxide synthase. J Biol Chem, 267, 11374-8.

Klatt, P., Pfeiffer, S., List, B.M., Lehner, D., Glatter, O., Bachinger, H.P., Werner, E.R., Schmidt, K. \& Mayer, B. (1996). Characterization of heme-deficient neuronal nitric-oxide synthase reveals a role for heme in subunit dimerization and binding of the amino acid substrate and tetrahydrobiopterin. J Biol Chem, 271, 7336-42.

Klatt, P., Schmid, M., Leopold, E., Schmidt, K., Werner, E.R. \& Mayer, B. (1994). The pteridine binding site of brain nitric oxide synthase. Tetrahydrobiopterin binding kinetics, specificity, and allosteric interaction with the substrate domain. J Biol Chem, 269, 13861-6.

Klatt, P., Schmidt, K., Lehner, D., Glatter, O., Bachinger, H.P. \& Mayer, B. (1995). Structural analysis of porcine brain nitric oxide synthase reveals a role for tetrahydrobiopterin and L-arginine in the formation of an SDS-resistant dimer. Embo $J, 14,3687-95$.

Klatt, P., Schmidt, K. \& Mayer, B. (1992). Brain nitric oxide synthase is a haemoprotein. Biochem J, 288, $15-7$.

Klatt, P., Schmidt, K., Uray, G. \& Mayer, B. (1993). Multiple catalytic functions of brain nitric oxide synthase. Biochemical characterization, cofactor-requirement, and the role of $\mathrm{N}$ omega-hydroxy-L-arginine as an intermediate. J Biol Chem, 268, 147817. 
Kleinert, H., Wallerath, T., Euchenhofer, C., Ihrig-Biedert, I., Li, H. \& Forstermann, U. (1998). Estrogens increase transcription of the human endothelial NO synthase gene: analysis of the transcription factors involved. Hypertension, 31, 582-8.

Kobzik, L., Bredt, D.S., Lowenstein, C.J., Drazen, J., Gaston, B., Sugarbaker, D. \& Stamler, J.S. (1993). Nitric oxide synthase in human and rat lung: immunocytochemical and histochemical localization. Am J Respir Cell Mol Biol, 9, 371-7.

Kobzik, L., Reid, M.B., Bredt, D.S. \& Stamler, J.S. (1994). Nitric oxide in skeletal muscle. Nature, 372, 546-8.

Kone, B.C. \& Kuncewicz, T. (1998). The Rho family GTPase Rac2 physically interacts with and activates inducible nitric oxide synthase. J. Am. Soc. Nephrol., 9, $463 \mathrm{a}$.

Konturek, J.W., Thor, P. \& Domschke, W. (1995). Effects of nitric oxide on antral motility and gastric emptying in humans. Eur J Gastroenterol Hepatol, 7, 97-102.

Krebs, E.G. \& Beavo, J.A. (1979). Phosphorylation-dephosphorylation of enzymes. Annu Rev Biochem, 48, 923-59.

Kroncke, K.D., Brenner, H.H., Rodriguez, M.L., Etzkorn, K., Noack, E.A., Kolb, H. \& Kolb-Bachofen, V. (1993). Pancreatic islet cells are highly susceptible towards the cytotoxic effects of chemically generated nitric oxide. Biochim Biophys Acta, 1182, 221-9.

Kroncke, K.D., Fehsel, K. \& Kolb-Bachofen, V. (1995). Inducible nitric oxide synthase and its product nitric oxide, a small molecule with complex biological activities. Biol Chem Hoppe Seyler, 376, 327-43.

Kroncke, K.D., Fehsel, K., Schmidt, T., Zenke, F.T., Dasting, I., Wesener, J.R., Bettermann, H., Breunig, K.D. \& Kolb-Bachofen, V. (1994). Nitric oxide destroys zinc-sulfur clusters inducing zinc release from metallothionein and inhibition of the zinc finger-type yeast transcription activator LAC9. Biochem Biophys Res Commun, 200, 1105-10.

Kroncke, K.D., Kolb-Bachofen, V., Berschick, B., Burkart, V. \& Kolb, H. (1991). Activated macrophages kill pancreatic syngeneic islet cells via arginine-dependent nitric oxide generation. Biochem Biophys Res Commun, 175, 752-8.

Krueger, J.K., Bishop, N.A., Blumenthal, D.K., Zhi, G., Beckingham, K., Stull, J.T. \& Trewhella, J. (1998). Calmodulin binding to myosin light chain kinase begins at substoichiometric $\mathrm{Ca} 2+$ concentrations: a small-angle scattering study of binding and conformational transitions. Biochemistry, 37, 17810-7. 
Kuchan, M.J. \& Frangos, J.A. (1994). Role of calcium and calmodulin in flowinduced nitric oxide production in endothelial cells. Am J Physiol, 266, C628-36.

Kwon, N.S., Nathan, C.F., Gilker, C., Griffith, O.W., Matthews, D.E. \& Stuehr, D.J. (1990). L-citrulline production from L-arginine by macrophage nitric oxide synthase. The ureido oxygen derives from dioxygen. $J$ Biol Chem, 265, 13442-5.

Kwon, N.S., Nathan, C.F. \& Stuehr, D.J. (1989). Reduced biopterin as a cofactor in the generation of nitrogen oxides by murine macrophages. J Biol Chem, 264, 20496501.

Kwon, N.S., Stuehr, D.J. \& Nathan, C.F. (1991). Inhibition of tumor cell ribonucleotide reductase by macrophage-derived nitric oxide. $J \operatorname{Exp} M e d, 174,761-7$.

Lamas, S., Marsden, P.A., Li, G.K., Tempst, P. \& Michel, T. (1992). Endothelial nitric oxide synthase: molecular cloning and characterization of a distinct constitutive enzyme isoform. Proc Natl Acad Sci U S A, 89, 6348-52.

Lancaster, J.R., Jr. (1994). Simulation of the diffusion and reaction of endogenously produced nitric oxide. Proc Natl Acad Sci U S A, 91, 8137-41.

Lander, H.M., Ogiste, J.S., Teng, K.K. \& Novogrodsky, A. (1995). p21 ras as a common signaling target of reactive free radicals and cellular redox stress. $J$ Biol Chem, 270, $21195-8$.

Lander, H.M., Sehajpal, P., Levine, D.M. \& Novogrodsky, A. (1993). Activation of human peripheral blood mononuclear cells by nitric oxide-generating compounds. $J$ Immunol, 150, 1509-16.

Lawrence, A.J., Krstew, E. \& Jarrott, B. (1997). Complex interactions between nitric oxide and adenosine receptors in the rat isolated nodose ganglion. Eur J Pharmacol, $328,83-8$.

Lee, C.G., Gregg, A.R. \& O'Brien, W.E. (1995). Localization of the neuronal form of nitric oxide synthase to mouse chromosome 5. Mamm Genome, 6, 56-7.

Lee, M.A., Cai, L., Hubner, N., Lee, Y.A. \& Lindpaintner, K. (1997). Tissue- and development-specific expression of multiple alternatively spliced transcripts of rat neuronal nitric oxide synthase. J Clin Invest, 100, 1507-12.

Lei, S.Z., Pan, Z.H., Aggarwal, S.K., Chen, H.S., Hartman, J., Sucher, N.J. \& Lipton, S.A. (1992). Effect of nitric oxide production on the redox modulatory site of the NMDA receptor-channel complex. Neuron, 8, 1087-99.

Leone, A.M., Palmer, R.M., Knowles, R.G., Francis, P.L., Ashton, D.S. \& Moncada, S. (1991). Constitutive and inducible nitric oxide synthases incorporate molecular oxygen into both nitric oxide and citrulline. J Biol Chem, 266, 23790-5.

Lepoivre, M., Chenais, B., Yapo, A., Lemaire, G., Thelander, L. \& Tenu, J.P. (1990). Alterations of ribonucleotide reductase activity following induction of the nitritegenerating pathway in adenocarcinoma cells. J Biol Chem, 265, 14143-9. 
Li, H., Oehrlein, S.A., Wallerath, T., Ihrig-Biedert, I., Wohlfart, P., Ulshofer, T., Jessen, T., Herget, T., Forstermann, U. \& Kleinert, H. (1998). Activation of protein kinase $\mathrm{C}$ alpha and/or epsilon enhances transcription of the human endothelial nitric oxide synthase gene. Mol Pharmacol, 53, 630-7.

Li, J., Billiar, T.R., Talanian, R.V. \& Kim, Y.M. (1997). Nitric oxide reversibly inhibits seven members of the caspase family via S-nitrosylation. Biochem Biophys Res Commun, 240, 419-24.

Lin, K.T., Xue, J.Y., Nomen, M., Spur, B. \& Wong, P.Y. (1995). Peroxynitriteinduced apoptosis in HL-60 cells. J Biol Chem, 270, 16487-90.

Lin, P.J., Chang, C.H. \& Chang, J.P. (1994). Reversal of refractory hypotension in septic shock by inhibitor of nitric oxide synthase. Chest, 106, 626-9.

Linse, S., Helmersson, A. \& Forsen, S. (1991). Calcium binding to calmodulin and its globular domains. J Biol Chem, 266, 8050-4.

Lipton, S.A., Choi, Y.B., Pan, Z.H., Lei, S.Z., Chen, H.S., Sucher, N.J., Loscalzo, J., Singel, D.J. \& Stamler, J.S. (1993). A redox-based mechanism for the neuroprotective and neurodestructive effects of nitric oxide and related nitroso-compounds. Nature, $364,626-32$.

List, B.M., Klosch, B., Volker, C., Gorren, A.C., Sessa; W.C., Werner, E.R., Kukovetz, W.R., Schmidt, K. \& Mayer, B. (1997). Characterization of bovine endothelial nitric oxide synthase as a homodimer with down-regulated uncoupled NADPH oxidase activity: tetrahydrobiopterin binding kinetics and role of haem in dimerization. Biochem J, 323, 159-65.

Liu, J., Garcia-Cardena, G. \& Sessa, W.C. (1995). Biosynthesis and palmitoylation of endothelial nitric oxide synthase: mutagenesis of palmitoylation sites, cysteines-15 and/or -26 , argues against depalmitoylation-induced translocation of the enzyme. Biochemistry, 34, 12333-40.

Liu, J., Garcia-Cardena, G. \& Sessa, W.C. (1996). Palmitoylation of endothelial nitric oxide synthase is necessary for optimal stimulated release of nitric oxide: implications for caveolae localization. Biochemistry, 35, 13277-81.

Liu, J. \& Sessa, W.C. (1994). Identification of covalently bound amino-terminal myristic acid in endothelial nitric oxide synthase. $J$ Biol Chem, 269, 11691-4.

Liu, Q. \& Gross, S.S. (1996). Binding sites of nitric oxide synthases. In Methods in Enzymology. ed. Packer, L. pp. 311-324. San Diego: Academic Press.

Liu, S.F., Ye, X. \& Malik, A.B. (1997). In vivo inhibition of nuclear factor-kappa B activation prevents inducible nitric oxide synthase expression and systemic hypotension in a rat model of septic shock. J Immunol, 159, 3976-83. 
Lorsbach, R.B., Murphy, W.J., Lowenstein, C.J., Snyder, S.H. \& Russell, S.W. (1993). Expression of the nitric oxide synthase gene in mouse macrophages activated for tumor cell killing. Molecular basis for the synergy between interferon-gamma and lipopolysaccharide. J Biol Chem, 268, 1908-13.

Los, M., Van de Craen, M., Penning, L.C., Schenk, H., Westendorp, M., Baeuerle, P.A., Droge, W., Krammer, P.H., Fiers, W. \& Schulze-Osthoff, K. (1995). Requirement of an ICE/CED-3 protease for Fas/APO-1-mediated apoptosis. Nature, 375, 81-3.

Lowe, P.N., Smith, D., Stammers, D.K., Riveros-Moreno, V., Moncada, S., Charles, I. \& Boyhan, A. (1996). Identification of the domains of neuronal nitric oxide synthase by limited proteolysis. Biochem $J, 314,55-62$.

Lowenstein, C.J., Alley, E.W., Raval, P., Snowman, A.M., Snyder, S.H., Russell, S.W. \& Murphy, W.J. (1993). Macrophage nitric oxide synthase gene: two upstream regions mediate induction by interferon gamma and lipopolysaccharide. Proc Natl Acad Sci U S A, 90, 9730-4.

Lundblad, R.L., Noyes, C.M., Featherstone, G.L., Harrison, J.H. \& Jenzano, J.W. (1988). The reaction of bovine alpha-thrombin with tetranitromethane. Characterization of the modified protein. J Biol Chem, 263, 3729-34.

Luo, Z., Fujio, Y., Kureishi, Y., Rudic, R.D., Daumerie, G., Fulton, D., Sessa, W.C. \& Walsh, K. (2000). Acute modulation of endothelial Akt/PKB activity alters nitric oxide-dependent vasomotor activity in vivo. J. Clin. Invest., 106, 493-499.

Lyons, C.R., Orloff, G.J. \& Cunningham, J.M. (1992). Molecular cloning and functional expression of an inducible nitric oxide synthase from a murine macrophage cell line. J Biol Chem, 267, 6370-4.

Macdonald, T.L., Gutheim, W.G., Martin, R.B. \& Guengerich, F.P. (1989). Oxidation of substituted N,N-dimethylanilines by cytochrome P-450: estimation of the effective oxidation-reduction potential of cytochrome P-450. Biochemistry, 28, 2071-7.

MacIntyre, I., Zaidi, M., Alam, A.S., Datta, H.K., Moonga, B.S., Lidbury, P.S., Hecker, M. \& Vane, J.R. (1991). Osteoclastic inhibition: an action of nitric oxide not mediated by cyclic GMP. Proc Natl Acad Sci U S A, 88, 2936-40.

MacNaul, K.L. \& Hutchinson, N.I. (1993). Differential expression of iNOS and cNOS mRNA in human vascular smooth muscle cells and endothelial cells under normal and inflammatory conditions. Biochem Biophys Res Commun, 196, 1330-4.

Magee, T., Fuentes, A.M., Garban, H., Rajavashisth, T., Marquez, D., Rodriguez, J.A., Rajfer, J. \& Gonzalez-Cadavid, N.F. (1996). Cloning of a novel neuronal nitric oxide synthase expressed in penis and lower urinary tract. Biochem Biophys Res Commun, 226, 145-51.

Mannick, J.B., Asano, K., Izumi, K., Kieff, E. \& Stamler, J.S. (1994). Nitric oxide produced by human $B$ lymphocytes inhibits apoptosis and Epstein-Barr virus reactivation. Cell, 79, 1137-46. 
Markewitz, B.A., Michael, J.R. \& Kohan, D.E. (1993). Cytokine-induced expression of a nitric oxide synthase in rat renal tubule cells. J Clin Invest, 91, 2138-43.

Marletta, M.A. (1993). Nitric oxide synthase structure and mechanism. J Biol Chem, $268,12231-4$.

Marletta, M.A., Yoon, P.S., Iyengar, R., Leaf, C.D. \& Wishnok, J.S. (1988). Macrophage oxidation of L-arginine to nitrite and nitrate: nitric oxide is an intermediate. Biochemistry, 27, 8706-11.

Marsden, P.A. \& Ballermann, B.J. (1990). Tumor necrosis factor alpha activates soluble guanylate cyclase in bovine glomerular mesangial cells via an L-argininedependent mechanism. $J$ Exp Med, 172, 1843-52.

Marsden, P.A., Heng, H.H., Duff, C.L., Shi, X.M., Tsui, L.C. \& Hall, A.V. (1994). Localization of the human gene for inducible nitric oxide synthase (NOS2) to chromosome 17q11.2-q12. Genomics, 19, 183-5.

Marsden, P.A., Heng, H.H., Scherer, S.W., Stewart, R.J., Hall, A.V., Shi, X.M., Tsui, L.C. \& Schappert, K.T. (1993). Structure and chromosomal localization of the human constitutive endothelial nitric oxide synthase gene. J Biol Chem, 268, 17478-88.

Marsden, P.A., Schappert, K.T., Chen, H.S., Flowers, M., Sundell, C.L., Wilcox, J.N., Lamas, S. \& Michel, T. (1992). Molecular cloning and characterization of human endothelial nitric oxide synthase. FEBS Lett, 307, 287-93.

Martasek, P., Liu, Q., Liu, J., Roman, L.J., Gross, S.S., Sessa, W.C. \& Masters, B.S. (1996). Characterization of bovine endothelial nitric oxide synthase expressed in $\mathrm{E}$. coli. Biochem Biophys Res Commun, 219, 359-65.

Martin, B.L., Wu, D., Jakes, S. \& Graves, D.J. (1990). Chemical influences on the specificity of tyrosine phosphorylation. $J$ Biol Chem, 265, 7108-11.

Masters, B.S., McMillan, K., Sheta, E.A., Nishimura, J.S., Roman, L.J. \& Martasek, P. (1996). Neuronal nitric oxide synthase, a modular enzyme formed by convergent evolution: structure studies of a cysteine thiolate-liganded heme protein that hydroxylates L-arginine to produce NO. as a cellular signal. Faseb J, 10, 552-8.

Masure, S., Haefner, B., Wesselink, J.J., Hoefnagel, E., Mortier, E., Verhasselt, P., Tuytelaars, A., Gordon, R. \& Richardson, A. (1999). Molecular cloning, expression and characterization of the human serine/threonine kinase Akt-3. Eur J Biochem, 265, p353-60.

Mayer, B., Heinzel, B., Klatt, P., John, M., Schmidt, K. \& Bohme, E. (1992). Nitric oxide synthase-catalyzed activation of oxygen and reduction of cytochromes: reaction mechanisms and possible physiological implications. J Cardiovasc Pharmacol, 20, uppl 12 pS54-6. 
Mayer, B., John, M., Heinzel, B., Werner, E.R., Wachter, H., Schultz, G. \& Bohme, E. (1991). Brain nitric oxide synthase is a biopterin- and flavin-containing multifunctional oxido-reductase. FEBS Lett, 288, 187-91.

McCabe, T.J., Fulton, D., Roman, L.J. \& Sessa, W.C. (2000). Enhanced electron flux and reduced calmodulin dissociation may explain "calcium-independent" eNOS activation by phosphorylation. J. Biol. Chem., 275, 6123-6128.

McCann, S.M., Kimura, M., Karanth, S., Yu, W.H. \& Rettori, V. (1997). Nitric oxide controls the hypothalamic-pituitary response to cytokines. Neuroimmunomodulation, 4, 98-106.

McDonald, K.K., Zharikov, S., Block, E.R. \& Kilberg, M.S. (1997). A caveolar complex between the cationic amino acid transporter 1 and endothelial nitric-oxide synthase may explain the "arginine paradox". J Biol Chem, 272, 31213-6.

McMillan, K., Bredt, D.S., Hirsch, D.J., Snyder, S.H., Clark, J.E. \& Masters, B.S. (1992). Cloned, expressed rat cerebellar nitric oxide synthase contains stoichiometric amounts of heme, which binds carbon monoxide. Proc Natl Acad Sci US A, 89, $11141-5$.

McMillan, K. \& Masters, B.S. (1995). Prokaryotic expression of the heme- and flavin-binding domains of rat neuronal nitric oxide synthase as distinct polypeptides: identification of the heme-binding proximal thiolate ligand as cysteine- 415 . Biochemistry, 34, 3686-93.

Melis, M.R. \& Argiolas, A. (1995). Nitric oxide donors induce penile erection and yawning when injected in the central nervous system of male rats. Eur J Pharmacol, 294, 1-9.

Messmer, U.K., Ankarcrona, M., Nicotera, P. \& Brune, B. (1994). p53 expression in nitric oxide-induced apoptosis. FEBS Lett, 355, 23-6.

Messmer, U.K. \& Brune, B. (1996). Nitric oxide-induced apoptosis: p53-dependent and p53-independent signalling pathways. Biochem J, 319, 299-305.

Messmer, U.K., Reed, U.K. \& Brune, B. (1996). Bcl-2 protects macrophages from nitric oxide-induced apoptosis. J Biol Chem, 271, 20192-7.

Messmer, U.K., Reimer, D.M., Reed, J.C. \& Brune, B. (1996). Nitric oxide induced poly(ADP-ribose) polymerase cleavage in RAW 264.7 macrophage apoptosis is blocked by Bcl-2. FEBS Lett, 384, 162-6.

Michel, J.B., Feron, O., Sacks, D. \& Michel, T. (1997). Reciprocal regulation of endothelial nitric-oxide synthase by $\mathrm{Ca} 2+$-calmodulin and caveolin. J Biol Chem, $272,15583-6$.

Michel, J.B., Feron, O., Sase, K., Prabhakar, P. \& Michel, T. (1997). Caveolin versus calmodulin. Counterbalancing allosteric modulators of endothelial nitric oxide synthase. J Biol Chem, 272, 25907-12. 
Michel, T., Li, G.K. \& Busconi, L. (1993). Phosphorylation and subcellular translocation of endothelial nitric oxide synthase. Proc Natl Acad Sci U S A, 90, 62526.

Michell, B.J., Griffiths, J.E., Mitchelhill, K.I., Rodriguez-Crespo, I., Tiganis, T., Bozinovski, S., de Montellano, P.R., Kemp, B.E. \& Pearson, R.B. (1999). The Akt kinase signals directly to endothelial nitric oxide synthase. Curr Biol, 9, 845-8.

Mitchell, H.H., Schonle, H.A. \& Grindy, H.S. (1916). The origin of nitrates in the urine. Journal of biological chemistry, 24, 461-490.

Mitchell, J.A., Hecker, M. \& Vane, J.R. (1990). The generation of L-arginine in endothelial cells is linked to the release of endothelium-derived relaxing factor. Eur $J$ Pharmacol, 176, 253-4.

Miwa, G.T., Walsh, J.S., Kedderis, G.L. \& Hollenberg, P.F. (1983). The use of intramolecular isotope effects to distinguish between deprotonation and hydrogen atom abstraction mechanisms in cytochrome P-450- and peroxidase-catalyzed $\mathrm{N}$ demethylation reactions. J Biol Chem, 258, 14445-9.

Miwa, M., Stuehr, D.J., Marletta, M.A., Wishnok, J.S. \& Tannenbaum, S.R. (1987). $\mathrm{N}$-nitrosamine formation by macrophages. IARC Sci Publ, 340-4.

Miyahara, K., Kawamoto, T., Sase, K., Yui, Y., Toda, K., Yang, L.X., Hattori, R., Aoyama, T., Yamamoto, Y., Doi, Y. \& et al. (1994). Cloning and structural characterization of the human endothelial nitric-oxide-synthase gene. Eur J Biochem, $223,719-26$.

Mohr, S., Stamler, J.S. \& Brune, B. (1994). Mechanism of covalent modification of glyceraldehyde-3-phosphate dehydrogenase at its active site thiol by nitric oxide, peroxynitrite and related nitrosating agents. FEBS Lett, 348, 223-7.

Mohr, S., Stamler, J.S. \& Brune, B. (1996). Posttranslational modification of glyceraldehyde-3-phosphate dehydrogenase by S-nitrosylation and subsequent NADH attachment. J Biol Chem, 271, 4209-14.

Molina y Vedia, L., McDonald, B., Reep, B., Brune, B., Di Silvio, M., Billiar, T.R. \& Lapetina, E.G. (1992). Nitric oxide-induced S-nitrosylation of glyceraldehyde-3phosphate dehydrogenase inhibits enzymatic activity and increases endogenous ADPribosylation [published erratum appears in J Biol Chem 1993 Feb 5; 268(4):3016]. J Biol Chem, 267, 24929-32.

Moncada, S., Palmer, R.M. \& Higgs, E.A. (1989). Biosynthesis of nitric oxide from L-arginine. A pathway for the regulation of cell function and communication. Biochem Pharmacol, 38, 1709-15. 
Montague, P.R., Gancayco, C.D., Winn, M.J., Marchase, R.B. \& Friedlander, M.J. (1994). Role of NO production in NMDA receptor-mediated neurotransmitter release in cerebral cortex. Science, 263, 973-7.

Montgomery, H.J., Romanov, V. \& Guillemette, J.G. (2000). Removal of a putative inhibitory element reduces the calcium dependent calmodulin activation of neuronal nitric oxide synthase. $J$ Biol Chem, 275, 5052-5058.

Morbidelli, L., Chang, C.H., Douglas, J.G., Granger, H.J., Ledda, F. \& Ziche, M. (1996). Nitric oxide mediates mitogenic effect of VEGF on coronary venular endothelium. Am J Physiol, 270, H411-5.

Morris, S.M., Jr. \& Billiar, T.R. (1994). New insights into the regulation of inducible nitric oxide synthesis. Am J Physiol, 266, E829-39.

Motterlini, R., Foresti, R., Intaglietta, M. \& Winslow, R.M. (1996). NO-mediated activation of heme oxygenase: endogenous cytoprotection against oxidative stress to endothelium. Am J Physiol, 270, H107-14.

Nakane, M., Mitchell, J., Forstermann, U. \& Murad, F. (1991). Phosphorylation by calcium calmodulin-dependent protein kinase II and protein kinase $\mathrm{C}$ modulates the activity of nitric oxide synthase. Biochem Biophys Res Commun, 180, 1396-402.

Nakatsuka, M. \& Osawa, Y. (1994). Selective inhibition of the 12-lipoxygenase pathway of arachidonic acid metabolism by L-arginine or sodium nitroprusside in intact human platelets. Biochem Biophys Res Commun, 200, 1630-4.

Nathan, C. (1995). Inducible nitric oxide synthase: regulation subserves function. Curr Top Microbiol Immunol, 196, 1-4.

Nathan, C. \& Xie, Q.W. (1994). Nitric oxide synthases: roles, tolls, and controls. Cell, 78, 915-8.

Nathan, C. \& Xie, Q.W. (1994). Regulation of biosynthesis of nitric oxide. J Biol Chem, 269, 13725-8.

Newton, D.C., Montgomery, H.J. \& Guillemette, J.G. (1998). The reductase domain of the human inducible nitric oxide synthase is fully active in the absence of bound calmodulin. Arch Biochem Biophys, 359, 249-57.

Nguyen, T., Brunson, D., Crespi, C.L., Penman, B.W., Wishnok, J.S. \& Tannenbaum, S.R. (1992). DNA damage and mutation in human cells exposed to nitric oxide in vitro. Proc Natl Acad Sci U S A, 89, 3030-4.

Nichol, C.A., Smith, G.K. \& Duch, D.S. (1985). Biosynthesis and metabolism of tetrahydrobiopterin and molybdopterin. Annu Rev Biochem, 54, 729-64.

Nicholson, D.W., Ali, A., Thornberry, N.A., Vaillancourt, J.P., Ding, C.K., Gallant, M., Gareau, Y., Griffin, P.R., Labelle, M., Lazebnik, Y.A. \& et al. (1995). 
Identification and inhibition of the ICE/CED-3 protease necessary for mammalian apoptosis. Nature, 376, 37-43.

Nikitovic, D. \& Holmgren, A. (1996). S-nitrosoglutathione is cleaved by the thioredoxin system with liberation of glutathione and redox regulating nitric oxide. $J$ Biol Chem, 271, 19180-5.

Nishida, C.R. \& Ortiz de Montellano, P.R. (1999). Autoinhibition of Endothelial nitric oxide synthase. Identification of an electron transfer control element. Journal of Biological Chemistry, 274, 14692-14698.

Nishida, C.R. \& Ortiz de Montellano, P.R. (1998). Electron transfer and catalytic activity of nitric oxide synthases. Chimeric constructs of the neuronal, inducible, and endothelial isoforms. J Biol Chem, 273, 5566-71.

Nishida, K., Harrison, D.G., Navas, J.P., Fisher, A.A., Dockery, S.P., Uematsu, M., Nerem, R.M., Alexander, R.W. \& Murphy, T.J. (1992). Molecular cloning and characterization of the constitutive bovine aortic endothelial cell nitric oxide synthase. J Clin Invest, 90, 2092-6.

Nishimura, J.S., Martasek, P., McMillan, K., Salerno, J., Liu, Q., Gross, S.S. \& Masters, B.S. (1995). Modular structure of neuronal nitric oxide synthase: localization of the arginine binding site and modulation by pterin. Biochem Biophys Res Commun, 210, 288-94.

Nixon, R.A. (1993). The regulation of neurofilament protein dynamics by phosphorylation: clues to neurofibrillary pathobiology. Brain Pathol, 3, 29-38.

Noiri, E., Peresleni, T., Srivastava, N., Weber, P., Bahou, W.F., Peunova, N. \& Goligorsky, M.S. (1996). Nitric oxide is necessary for a switch from stationary to locomoting phenotype in epithelial cells. Am J Physiol, 270, C794-802.

Nunokawa, Y., Ishida, N. \& Tanaka, S. (1994). Promoter analysis of human inducible nitric oxide synthase gene associated with cardiovascular homeostasis. Biochem Biophys Res Commun, 200, 802-7.

Nussler, A.K., Di Silvio, M., Billiar, T.R., Hoffman, R.A., Geller, D.A., Selby, R., Madariaga, J. \& Simmons, R.L. (1992). Stimulation of the nitric oxide synthase pathway in human hepatocytes by cytokines and endotoxin. $J$ Exp Med, 176, 261-4.

O'Dowd, B.F., Hnatowich, M., Caron, M.G., Lefkowitz, R.J. \& Bouvier, M. (1989). Palmitoylation of the human beta 2-adrenergic receptor. Mutation of Cys341 in the carboxyl tail leads to an uncoupled nonpalmitoylated form of the receptor. $J$ Biol Chem, 264, 7564-9.

O'Halloran, T.V. (1993). Transition metals in control of gene expression. Science, $261,715-25$.

O'Neil, K.T. \& De Grado, W.F. (1990). How calmodulin binds its targets: sequence independent recognition of amphiphilic alpha-helices. Trends Biochem Sci, 15, 59-64. 
Ogura, T., Yokoyama, T., Fujisawa, H., Kurashima, Y. \& Esumi, H. (1993). Structural diversity of neuronal nitric oxide synthase mRNA in the nervous system. Biochem Biophys Res Commun, 193, 1014-22.

Orihata, M. \& Sarna, S.K. (1994). Inhibition of nitric oxide synthase delays gastric emptying of solid meals. J Pharmacol Exp Ther, 271, 660-70.

Padmaja, S. \& Huie, R.E. (1993). The reaction of nitric oxide with organic peroxyl radicals. Biochem Biophys Res Commun, 195, 539-44.

Palacios, M., Knowles, R.G., Palmer, R.M. \& Moncada, S. (1989). Nitric oxide from L-arginine stimulates the soluble guanylate cyclase in adrenal glands. Biochem Biophys Res Commun, 165, 802-9.

Palmer, R.M., Ashton, D.S. \& Moncada, S. (1988). Vascular endothelial cells synthesize nitric oxide from L-arginine. Nature, 333, 664-6.

Palmer, R.M., Ferrige, A.G. \& Moncada, S. (1987). Nitric oxide release accounts for the biological activity of endothelium-derived relaxing factor. Nature, 327, 524-6.

Palmer, R.M. \& Moncada, S. (1989). A novel citrulline-forming enzyme implicated in the formation of nitric oxide by vascular endothelial cells. Biochem Biophys Res Commun, 158, p348-52.

Pantopoulos, K. \& Hentze, M.W. (1995). Nitric oxide signaling to iron-regulatory protein: direct control of ferritin mRNA translation and transferrin receptor mRNA stability in transfected fibroblasts. Proc Natl Acad Sci U S A, 92, 1267-71.

Persechini, A. \& Cronk, B. (1999). The relationship between the free concentrations of $\mathrm{Ca} 2+$ and $\mathrm{Ca} 2+$-calmodulin in intact cells. $\mathrm{J}$ Biol Chem, 274, 6827-30.

Persechini, A., Gansz, K.J. \& Paresi, R.J. (1996). Activation of myosin light chain kinase and nitric oxide synthase activities by engineered calmodulins with duplicated or exchanged EF hand pairs. Biochemistry, 35, 224-8.

Persechini, A., Gansz, K.J. \& Paresi, R.J. (1996). A role in enzyme activation for the N-terminal leader sequence in calmodulin. J Biol Chem, 271, 19279-82.

Persechini, A., McMillan, K. \& Leakey, P. (1994). Activation of myosin light chain kinase and nitric oxide synthase activities by calmodulin fragments. J Biol Chem, 269, 16148-54.

Persechini, A., Moncrief, N.D. \& Kretsinger, R.H. (1989). The EF-hand family of calcium-modulated proteins. Trends Neurosci, 12, 462-7.

Persechini, A., White, H.D. \& Gansz, K.J. (1996). Different mechanisms for Ca2+ dissociation from complexes of calmodulin with nitric oxide synthase or myosin light chain kinase. J Biol Chem, 271, 62-7. 
Petros, A., Bennett, D. \& Vallance, P. (1991). Effect of nitric oxide synthase inhibitors on hypotension in patients with septic shock. Lancet, 338, 1557-8.

Peunova, N. \& Enikolopov, G. (1995). Nitric oxide triggers a switch to growth arrest during differentiation of neuronal cells. Nature, 375, 68-73.

Pfeiffer, S. \& Mayer, B. (1998). Lack of tyrosine nitration by peroxynitrite generated at physiological pH. J. Biol. Chem., 273, 27280-27285.

Pfeilschifter, J. \& Huwiler, A. (1996). Nitric oxide stimulates stress-activated protein kinases in glomerular endothelial and mesangial cells. FEBS Lett, 396, 67-70.

Plourde, V., Quintero, E., Suto, G., Coimbra, C. \& Tache, Y. (1994). Delayed gastric emptying induced by inhibitors of nitric oxide synthase in rats. Eur J Pharmacol, 256, 125-9.

Pollock, J.S., Forstermann, U., Mitchell, J.A., Warner, T.D., Schmidt, H.H., Nakane, M. \& Murad, F. (1991). Purification and characterization of particulate endotheliumderived relaxing factor synthase from cultured and native bovine aortic endothelial cells. Proc Natl Acad Sci U S A, 88, 10480-4.

Pollock, J.S., Nakane, M., Forstermann, U. \& Murad, F. (1992). Particulate and soluble bovine endothelial nitric oxide synthases are structurally similar proteins yet different from soluble brain nitric oxide synthase. J Cardiovasc Pharmacol, 20, S503.

Porter, T.D. (1991). An unusual yet strongly conserved flavoprotein reductase in bacteria and mammals. Trends Biochem Sci, 16, 154-8.

Pou, S., Pou, W.S., Bredt, D.S., Snyder, S.H. \& Rosen, G.M. (1992). Generation of superoxide by purified brain nitric oxide synthase. J Biol Chem, 267, 24173-6.

Prickaerts, J., Steinbusch, H.W., Smits, J.F. \& de Vente, J. (1997). Possible role of nitric oxide-cyclic GMP pathway in object recognition memory: effects of 7 nitroindazole and zaprinast. Eur J Pharmacol, 337, 125-36.

Pufahl, R.A. \& Marletta, M.A. (1993). Oxidation of NG-hydroxy-L-arginine by nitric oxide synthase: evidence for the involvement of the heme in catalysis. Biochem Biophys Res Commun, 193, 963-70.

Radi, R., Beckman, J., Bush, K. \& et al (1991). Peroxy nitrite-induced membrane lipid peroxidation: the cytotoxic potential of superoxide and nitric oxide. Arch Biochem Biopys, 288, 481-7.

Radi, R., Beckman, J.S., Bush, K.M. \& Freeman, B.A. (1991). Peroxynitrite oxidation of sulfhydryls. The cytotoxic potential of superoxide and nitric oxide. $J$ Biol Chem, $266,4244-50$. 
Radomski, M.W., Palmer, R.M. \& Moncada, S. (1990). Characterization of the Larginine:nitric oxide pathway in human platelets. Br J Pharmacol, 101, 325-8.

Ratovitski, E., Bao, C., Quick, R., McMillan, A., Kozlovsky, C. \& Lowenstein, C. (1999). An inducible nitric-oxide synthase (NOS)-associated protein inhibits NOS dimerization and activity. $J$ Biol Chem, 274, p30250-7.

Regulski, M. \& Tully, T. (1995). Molecular and biochemical characterization of dNOS: a Drosophila Ca2+/calmodulin-dependent nitric oxide synthase. Proc Natl Acad Sci U S A, 92, 9072-6.

Reif, D.W. \& Simmons, R.D. (1990). Nitric oxide mediates iron release from ferritin. Arch Biochem Biophys, 283, 537-41.

Renaud, J.P., Boucher, J.L., Vadon, S., Delaforge, M. \& Mansuy, D. (1993). Particular ability of liver P450s3A to catalyze the oxidation of $\mathrm{N}$ omegahydroxyarginine to citrulline and nitrogen oxides and occurrence in no synthases of a sequence very similar to the heme-binding sequence in P450s. Biochem Biophys Res Commun, 192, 53-60.

Resh, M.D. (1994). Myristylation and palmitylation of Src family members: the fats of the matter. Cell, 76, 411-3.

Resh, M.D. (1996). Regulation of cellular signalling by fatty acid acylation and prenylation of signal transduction proteins. Cell Signal, 8, 403-12.

Rettori, V., Belova, N., Dees, W.L., Nyberg, C.L., Gimeno, M. \& McCann, S.M. (1993). Role of nitric oxide in the control of luteinizing hormone-releasing hormone release in vivo and in vitro. Proc Natl Acad Sci U S A, 90, 10130-4.

Richards, M.K., Clague, M.J. \& Marletta, M.A. (1996). Characterization of C415 mutants of neuronal nitric oxide synthase. Biochemistry, 35, 7772-80.

Rickard, N.S., Ng, K.T. \& Gibbs, M.E. (1998). Further support for nitric oxidedependent memory processing in the day-old chick. Neurobiol Learn Mem, 69, 79-86.

Rimele, T.J., Sturm, R.J., Adams, L.M., Henry, D.E., Heaslip, R.J., Weichman, B.M. \& Grimes, D. (1988). Interaction of neutrophils with vascular smooth muscle: identification of a neutrophil-derived relaxing factor. $J$ Pharmacol Exp Ther, 245, $102-11$.

Robinson, L.J., Busconi, L. \& Michel, T. (1995). Agonist-modulated palmitoylation of endothelial nitric oxide synthase. J Biol Chem, 270, 995-8.

Robinson, L.J. \& Michel, T. (1995). Mutagenesis of palmitoylation sites in endothelial nitric oxide synthase identifies a novel motif for dual acylation and subcellular targeting. Proc Natl Acad Sci U S A, 92, 11776-80. 
Robinson, L.J., Weremowicz, S., Morton, C.C. \& Michel, T. (1994). Isolation and chromosomal localization of the human endothelial nitric oxide synthase (NOS3) gene. Genomics, 19, 350-7.

Rodriguez-Crespo, I., Gerber, N.C. \& Ortiz de Montellano, P.R. (1996). Endothelial nitric-oxide synthase. Expression in Escherichia coli, spectroscopic characterization, and role of tetrahydrobiopterin in dimer formation. J Biol Chem, 271, 11462-7.

Rodriguez-Crespo, I. \& Ortiz de Montellano, P.R. (1996). Human endothelial nitric oxide synthase: expression in Escherichia coli, coexpression with calmodulin, and characterization. Arch Biochem Biophys, 336, 151-6.

Roman, L.J., Martasek, P., Miller, R.T., Harris, D.E., de La Garza, M.A., Shea, T.M., Kim, J.J. \& Masters, B.S. (2000). The C-terminus of constitutive nitric oxide synthases controls electron Flow through the flavin and heme domains and affects modulation by calmodulin. [Record Supplied By Publisher]. J Biol Chem, 27.

Roman, L.J., Miller, R.T., de La Garza, M.A., Kim, J.J. \& Siler Masters, B.S. (2000). The $C$ terminus of mouse macrophage inducible nitric-oxide synthase attenuates electron flow through the flavin domain. J Biol Chem, 275, p21914-9.

Roman, L.J., Sheta, E.A., Martasek, P., Gross, S.S., Liu, Q. \& Masters, B.S. (1995). High-level expression of functional rat neuronal nitric oxide synthase in Escherichia coli. Proc Natl Acad Sci U S A, 92, 8428-32.

Rossmann, M.G., Moras, D. \& Olsen, K.W. (1974). Chemical and biological evolution of nucleotide-binding protein. Nature, 250, 194-9.

Ruan, J., Xie, Q.-w., Hutchinson, N., Cho, H., Wolfe, G.C. \& Nathan, C. (1996). Inducible nitric oxide synthase reguires both the canonical calmodulin-binding domain and additional sequences in order to bind calmodulin and produce nitric oxide in the absence of free calcium. J. Biol. Chem., 271, 22679-22686.

Rubbo, H., Radi, R., Trujillo, M., Telleri, R., Kalyanaraman, B., Barnes, S., Kirk, M. \& Freeman, B.A. (1994). Nitric oxide regulation of superoxide and peroxynitritedependent lipid peroxidation. Formation of novel nitrogen-containing oxidized lipid derivatives. J Biol Chem, 269, 26066-75.

Ruiz, F., Corrales, F.J., Miqueo, C. \& Mato, J.M. (1998). Nitric oxide inactivates rat hepatic methionine adenosyltransferase in vivo by S-nitrosylation. Hepatology, 28, 1051-1057.

Sakuma, I., Stuehr, D.J., Gross, S.S., Nathan, C. \& Levi, R. (1988). Identification of arginine as a precursor of endothelium-derived relaxing factor. Proc Natl Acad Sci $U$ $S A, 85,8664-7$.

Salerno, J.C., Frey, C., McMillan, K., Williams, R.F., Masters, B.S. \& Griffith, O.W. (1995). Characterization by electron paramagnetic resonance of the interactions of $L$ - 
arginine and L-thiocitrulline with the heme cofactor region of nitric oxide synthase. $J$ Biol Chem, 270, 27423-8.

Salerno, J.C., Harris, D.E., Irizarry, K., Patel, B., Morales, A.J., Smith, S.M., Martasek, P., Roman, L.J., Masters, B.S., Jones, C.L., Weissman, B.A., Lane, P., Liu, Q. \& Gross, S.S. (1997). An autoinhibitory control element defines calcium-regulated isoforms of nitric oxide synthase. J Biol Chem, 272, 29769-77.

Salerno, J.C., Martasek, P., Roman, L.J. \& Masters, B.S. (1996). Electron paramagnetic resonance spectroscopy of the heme domain of inducible nitric oxide synthase: binding of ligands at the arginine site induces changes in the heme ligation geometry. Biochemistry, 35, 7626-30.

Salerno, J.C., McMillan, K. \& Masters, B.S. (1996). Binding of intermediate, product, and substrate analogs to neuronal nitric oxide synthase: ferriheme is sensitive to ligand-specific effects in the L-arginine binding site. Biochemistry, 35, 11839-45.

Salgo, M.G., Squadrito, G.L. \& Pryor, W.A. (1995). Peroxynitrite causes apoptosis in rat thymocytes. Biochem Biophys Res Commun, 215, 1111-8.

Salvemini, D., Misko, T.P., Masferrer, J.L., Seibert, K., Currie, M.G. \& Needleman, P. (1993). Nitric oxide activates cyclooxygenase enzymes. Proc Natl Acad Sci U S A, $90,7240-4$.

Sandau, K., Pfeilschifter, J. \& Brune, B. (1997). The balance between nitric oxide and superoxide determines apoptotic and necrotic death of rat mesangial cells. J Immunol, $158,4938-46$.

Sano, H., Hirai, M., Saito, H., Nakashima, I. \& Isobe, K. (1996). Nitric oxide releasing reagent, $\mathrm{S}$-nitroso- $\mathrm{N}$-acetylpenicillamine, enhances the expression of manganese superoxide dismutase mRNA in rat vascular smooth muscle cells. $J$ Cell Biochem, 62, 50-5.

Sarih, M., Souvannavong, V. \& Adam, A. (1993). Nitric oxide synthase induces macrophage death by apoptosis. Biochem Biophys Res Commun, 191, 503-8.

Scherrer, U., Randin, D., Vollenweider, P., Vollenweider, L. \& Nicod, P. (1994). Nitric oxide release accounts for insulin's vascular effects in humans. J Clin Invest, 94, 2511-5.

Schlegel, A., Volonte, D., Engelman, J.A., Galbiati, F., Mehta, P., Zhang, X.L., Scherer, P.E. \& Lisanti, M.P. (1998). Crowded little caves: structure and function of caveolae. Cell Signal, 10, 457-63.

Schmidt, H.H., Klein, M.M., Niroomand, F. \& Bohme, E. (1988). Is arginine a physiological precursor of endothelium-derived nitric oxide? Eur J Pharmacol, 148, 293-5. 
Schmidt, H.H., Nau, H., Wittfoht, W., Gerlach, J., Prescher, K.E., Klein, M.M., Niroomand, F. \& Bohme, E. (1988). Arginine is a physiological precursor of endothelium-derived nitric oxide. Eur J Pharmacol, 154, 213-6.

Schmidt, H.H., Smith, R.M., Nakane, M. \& Murad, F. (1992). Ca2+/calmodulindependent NO synthase type I: a biopteroflavoprotein with $\mathrm{Ca} 2+/$ calmodulinindependent diaphorase and reductase activities. Biochemistry, 31, 3243-9.

Schmidt, H.H.H.W., Lohman, S.M. \& Walter, U. (1993). The nitric oxide and cGMPsignal transduction system: regulation and mechanism of action. Biochem Biophys Acta, 1178, 153-75.

Schuppe-Koistinen, I., Moldeus, P., Bergman, T. \& Cotgreave, I.A. (1994). Sthiolation of human endothelial cell glyceraldehyde-3-phosphate dehydrogenase after hydrogen peroxide treatment. Eur J Biochem, 221, 1033-7.

Schwarz, M.A., Lazo, J.S., Yalowich, J.C., Allen, W.P., Whitmore, M., Bergonia, H.A., Tzeng, E., Billiar, T.R., Robbins, P.D., Lancaster, J.R., Jr. \& et al. (1995). Metallothionein protects against the cytotoxic and DNA-damaging effects of nitric oxide. Proc Natl Acad Sci U S A, 92, 4452-6.

Seilicovich, A., Duvilanski, B.H., Pisera, D., Theas, S., Gimeno, M., Rettori, V. \& McCann, S.M. (1995). Nitric oxide inhibits hypothalamic luteinizing hormonereleasing hormone release by releasing gamma-aminobutyric acid. Proc Natl Acad Sci $U S A, 92,3421-4$.

Sessa, W.C. (1994). The nitric oxide synthase family of proteins. J Vasc Res, 31, 13143.

Sessa, W.C., Barber, C.M. \& Lynch, K.R. (1993). Mutation of N-myristoylation site converts endothelial cell nitric oxide synthase from a membrane to a cytosolic protein. Circ Res, 72, 921-4.

Sessa, W.C., Harrison, J.K., Barber, C.M., Zeng, D., Durieux, M.E., D'Angelo, D.D., Lynch, K.R. \& Peach, M.J. (1992). Molecular cloning and expression of a cDNA encoding endothelial cell nitric oxide synthase. J Biol Chem, 267, 15274-6.

Shaul, P.W. \& Anderson, R.G. (1998). Role of plasmalemmal caveolae in signal transduction. Am J Physiol, 275, L843-51.

Shaul, P.W., Smart, E.J., robinson, L., German, Z., Yuhanna, I.S., Ying, Y., Anderson, R.G. \& Michel, T. (1996). Acylation targets endothelial nitric-oxide synthase to plasmalemmal caveolae. J Biol Chem, 271, 6518-22.

Sherman, P.A., Laubach, V.E., Reep, B.R. \& Wood, E.R. (1993). Purification and cDNA sequence of an inducible nitric oxide synthase from a human tumor cell line. Biochemistry, 32, 11600-5. 
Sheta, E.A., McMillan, K. \& Masters, B.S. (1994). Evidence for a bidomain structure of constitutive cerebellar nitric oxide synthase. J Biol Chem, 269, 15147-53.

Silvagno, F., Xia, H. \& Bredt, D.S. (1996). Neuronal nitric-oxide synthase-mu, an alternatively spliced isoform expressed in differentiated skeletal muscle. J Biol Chem, $271,11204-8$.

Sirsjo, A., Soderkvist, P., Sundqvist, T., Carlsson, M., Ost, M. \& Gidlof, A. (1994). Different induction mechanisms of mRNA for inducible nitric oxide synthase in rat smooth muscle cells in culture and in aortic strips. FEBS Lett, 338, 191-6.

Stadler, J., Stefanovic-Racic, M., Billiar, T.R., Curran, R.D., McIntyre, L.A., Georgescu, H.I., Simmons, R.L. \& Evans, C.H. (1991). Articular chondrocytes synthesize nitric oxide in response to cytokines and lipopolysaccharide. J Immunol, $147,3915-20$.

Stadler, J., Trockfeld, J., Schmalix, W.A., Brill, T., Siewert, J.R., Greim, H. \& Doehmer, J. (1994). Inhibition of cytochromes P4501A by nitric oxide. Proc Natl Acad Sci U S A, 91, 3559-63.

Stamler, J.S. (1994). Redox signaling: nitrosylation and related target interactions of nitric oxide. Cell, 78, 931-6.

Stamler, J.S., Singel, D.J. \& Loscalzo, J. (1992). Biochemistry of nitric oxide and its redox-activated forms. Science, 258, 1898-902.

Steller, H. (1995). Mechanisms and genes of cellular suicide. Science, 267, 1445-9. Stevens-Truss, R. \& Marletta, M.A. (1995). Interaction of calmodulin with the inducible murine macrophage nitric oxide synthase. Biochemistry, 34, 15638-45.

Stoyanovsky, D., Murphy, T., Anno, P.R., Kim, Y.M. \& Salama, G. (1997). Nitric oxide activates skeletal and cardiac ryanodine receptors. Cell Calcium, 21, 19-29.

Strack, S., Barban, M.A., Wadzinski, B.E. \& Colbran, R.J. (1997). Differential inactivation of postsynaptic density-associated and soluble $\mathrm{Ca} 2+/$ calmodulindependent protein kinase II by protein phosphatases 1 and $2 \mathrm{~A}$. Journal of Neurochemistry, 68, 2119-2228.

Stuehr, D.J. (1997). Structure-function aspects in the nitric oxide synthases. Annu Rev Pharmacol Toxicol, 37, 339-59.

Stuehr, D.J., Cho, H.J., Kwon, N.S., Weise, M.F. \& Nathan, C.F. (1991). Purification and characterization of the cytokine-induced macrophage nitric oxide synthase: an FAD- and FMN-containing flavoprotein. Proc Natl Acad Sci U S A, 88, 7773-7.

Stuehr, D.J. \& Griffith, O.W. (1992). Mammalian nitric oxide synthases. $A d v$ Enzymol Relat Areas Mol Biol, 65, 287-346. 
Stuehr, D.J., Gross, S.S., Sakuma, I., Levi, R. \& Nathan, C.F. (1989). Activated murine macrophages secrete a metabolite of arginine with the bioactivity of endothelium-derived relaxing factor and the chemical reactivity of nitric oxide. $J$ Exp Med, 169, 1011-20.

Stuehr, D.J. \& Ikeda-Saito, M. (1992). Spectral characterization of brain and macrophage nitric oxide synthases. Cytochrome P-450-like hemeproteins that contain a flavin semiquinone radical. $J$ Biol Chem, 267, 20547-50.

Stuehr, D.J., Kwon, N.S., Nathan, C.F., Griffith, O.W., Feldman, P.L. \& Wiseman, J. (1991). $\mathrm{N}$ omega-hydroxy-L-arginine is an intermediate in the biosynthesis of nitric oxide from L-arginine. J Biol Chem, 266, 6259-63.

Stuehr, D.J. \& Marletta, M.A. (1985). Mammalian nitrate biosynthesis: mouse macrophages produce nitrite and nitrate in response to Escherichia coli lipopolysaccharide. Proc Natl Acad Sci U S A, 82, 7738-42.

Stuehr, D.J. \& Nathan, C.F. (1989). Nitric oxide. A macrophage product responsible for cytostasis and respiratory inhibition in tumor target cells. J Exp Med, 169, 154355.

Suschek, C., Rothe, H., Fehsel, K., Enczmann, J. \& Kolb-Bachofen, V. (1993). Induction of a macrophage-like nitric oxide synthase in cultured rat aortic endothelial cells. IL-1 beta-mediated induction regulated by tumor necrosis factor-alpha and IFNgamma. J Immunol, 151, 3283-91.

Swain, J.A., Darley-Usmar, V. \& Gutteridge, J.M. (1994). Peroxynitrite releases copper from caeruloplasmin: implications for atherosclerosis. FEBS Lett, 342, 49-52.

Szabo, C. \& Thiemermann, C. (1995). Regulation of the expression of the inducible isoform of nitric oxide synthase. Adv Pharmacol, 34, 113-53.

Takakura, K., Hasegawa, K., Goto, Y. \& Muramatsu, I. (1997). Nitric oxide produced by inducible nitric oxide synthase delays gastric emptying in lipopolysaccharidetreated rats. Anesthesiology, 87, 652-7.

Tannenbaum, S.R., Fett, D., Young, V.R., Land, P.D. \& Bruce, W.R. (1978). Nitrite and nitrate are formed by endogenous synthesis in the human intestine. Science, 200, 1487-9.

Taylor, P.D. \& Poston, L. (1994). The effect of hyperglycaemia on function of rat isolated mesenteric resistance artery. Br J Pharmacol, 113, p801-8.

Tiao, G., Rafferty, J., Ogle, C., Fischer, J.E. \& Hasselgren, P.O. (1994). Detrimental effect of nitric oxide synthase inhibition during endotoxemia may be caused by high levels of tumor necrosis factor and interleukin-6. Surgery, 116, 332-7; discussion 3378. 
Tojo, A., Bredt, D.S. \& Wilcox, C.S. (1999). Distribution of postsynaptic density proteins in rat kidney: relationship to neuronal nitric oxide synthase. Kidney International, 55, 1384-1394.

Trigo-Rocha, F., Aronson, W.J., Hohenfellner, M., Ignarro, L.J., Rajfer, J. \& Lue, T.F. (1993). Nitric oxide and cGMP: mediators of pelvic nerve-stimulated erection in dogs. Am J Physiol, 264, H419-22.

Tsukahara, H., Gordienko, D.V., Tonshoff, B., Gelato, M.C. \& Goligorsky, M.S. (1994). Direct demonstration of insulin-like growth factor-I-induced nitric oxide production by endothelial cells. Kidney Int, 45, 598-604.

van der Veen, R.C., Hinton, D.R., Incardonna, F. \& Hofman, F.M. (1997). Extensive peroxynitrite activity during progressive stages of central nervous system inflammation. J Neuroimmunol, 77, 1-7.

Venema, R.C., Nishida, K., Alexander, R.W., Harrison, D.G. \& Murphy, T.J. (1994). Organization of the bovine gene encoding the endothelial nitric oxide synthase. Biochim Biophys Acta, 1218, 413-20.

Venema, R.C., Sayegh, H.S., Kent, J.D. \& Harrison, D.G. (1996). Identification, charaterization, and comparison of the calmodulin-binding domains of the endothelial and inducible nitric oxide sythases. $J$ Biol Chem, 271, 6435-40.

Venema, V.J., Marrero, M.B. \& Venema, R.C. (1996). Bradykinin-stimulated protein tyrosine phosphorylation promotes endothelial nitric oxide synthase translocation to the cytoskeleton. Biochem Biophys Res Commun, 226, 703-10.

Venema, V.J., Zou, R., Ju, H., Marrero, M.B. \& Venema, R.C. (1997). Caveolin-1 detergent solubility and association with endothelial nitric oxide synthase is modulated by tyrosine phosphorylation. Biochem Biophys Res Commun, 236, 155-61. Vodovotz, Y., Russell, D., Xie, Q.W., Bogdan, C. \& Nathan, C. (1995). Vesicle membrane association of nitric oxide synthase in primary mouse macrophages. $J$ Immunol, 154, 2914-25.

Vorherr, T., Knopfel, L., Hofmann, F., Mollner, S., Pfeuffer, T. \& Carafoli, E. (1993). The calmodulin binding domain of nitric oxide synthase and adenylyl cyclase. Biochemistry, 32, 6081-8.

Wagner, D.A., Young, V.R. \& Tannenbaum, S.R. (1983). Mammalian nitrate biosynthesis: incorporation of $15 \mathrm{NH} 3$ into nitrate is enhanced by endotoxin treatment. Proc Natl Acad Sci U S A, 80, 4518-21.

Walker, M.W., Kinter, M.T., Roberts, R.J. \& Spitz, D.R. (1995). Nitric oxide-induced cytotoxicity: involvement of cellular resistance to oxidative stress and the role of glutathione in protection. Pediatr Res, 37, 41-9.

Wang, J., Stuehr, D.J., Ikeda-Saito, M. \& Rousseau, D.L. (1993). Heme coordination and structure of the catalytic site in nitric oxide synthase. J Biol Chem, 268, 22255-8. 
Wang, M., Roberts, D.L., Paschke, R., Shea, T.M., Masters, B.S. \& Kim, J.J. (1997). Three-dimensional structure of NADPH-cytochrome P450 reductase: prototype for FMN- and FAD-containing enzymes. Proc Natl Acad Sci U S A, 94, 8411-6.

Wang, Y., Goligorsky, M.S., Lin, M., Wilcox, J.N. \& Marsden, P.A. (1997). A novel, testis-specific mRNA transcript encoding an NH2-terminal truncated nitric-oxide synthase. J Biol Chem, 272, 11392-401.

Wang, Y. \& Marsden, P.A. (1995). Nitric oxide synthases: gene structure and regulation. Adv Pharmacol, 34, 71-90.

Watanabe, K., Hirahashi, N., Narimatsu, S., Yamamoto, I. \& Yoshimura, H. (1991). Mouse hepatic microsomal enzyme that catalyzes oxidation of 11-oxo-delta 8tetrahydrocannabinol to delta 8-tetrahydrocannabinol-11-oic acid. Drug Metab Dispos, 19, 218-21.

Watanabe, Y., Terashima, K. \& Hidaka, H. (1996). Neuronal nitric oxide synthase specific autophosphorylation in baculovirus/Sf9 insect cell system. Biochem Biophys Res Commun, 219, 638-43.

Watenpaugh, K.D., Sieker, L.C. \& Jensen, L.H. (1973). The binding of riboflavin-5'phosphate in a flavoprotein: flavodoxin at 2.0-Angstrom resolution. Proc Natl Acad Sci U S A, 70, 3857-60.

Wedegaertner, P.B. \& Bourne, H.R. (1994). Activation and depalmitoylation of Gs alpha. Cell, 77, 1063-70.

Wedegaertner, P.B., Wilson, P.T. \& Bourne, H.R. (1995). Lipid modifications of trimeric G proteins. J Biol Chem, 270, 503-6.

Weinstein, H. \& Mehler, E.L. (1994). Ca(2+)-binding and structural dynamics in the functions of calmodulin. Annu Rev Physiol, 56, 213-36.

Weiss, G., Goossen, B., Doppler, W., Fuchs, D., Pantopoulos, K., Werner-Felmayer, G., Wachter, H. \& Hentze, M.W. (1993). Translational regulation via iron-responsive elements by the nitric oxide/NO-synthase pathway. Embo J, 12, 3651-7.

Wharton, M., Granger, D.L. \& Durack, D.T. (1988). Mitochondrial iron loss from leukemia cells injured by macrophages. A possible mechanism for electron transport chain defects. J Immunol, 141, 1311-7.

White, C.R., Brock, T.A., Chang, L.Y., Crapo, J., Briscoe, P., Ku, D., Bradley, W.A., Gianturco, S.H., Gore, J., Freeman, B.A. \& et al. (1994). Superoxide and peroxynitrite in atherosclerosis. Proc Natl Acad Sci U S A, 91, 1044-8. 
White, K.A. \& Marletta, M.A. (1992). Nitric oxide synthase is a cytochrome P-450 type hemoprotein. Biochemistry, 31, 6627-31.

Wink, D.A., Darbyshire, J.F., Nims, R.W., Saavedra, J.E. \& Ford, P.C. (1993). Reactions of the bioregulatory agent nitric oxide in oxygenated aqueous media: determination of the kinetics for oxidation and nitrosation by intermediates generated in the NO/O2 reaction. Chem Res Toxicol, 6, 23-7.

Wink, D.A., Kasprzak, K.S., Maragos, C.M., Elespuru, R.K., Misra, M., Dunams, T.M., Cebula, T.A., Koch, W.H., Andrews, A.W., Allen, J.S. \& et al. (1991). DNA deaminating ability and genotoxicity of nitric oxide and its progenitors. Science, 254, 1001-3.

Wood, E.R., Berger, H., Jr., Sherman, P.A. \& Lapetina, E.G. (1993). Hepatocytes and macrophages express an identical cytokine inducible nitric oxide synthase gene. Biochem Biophys Res Commun, 191, 767-74.

Wright, C.D., Mulsch, A., Busse, R. \& Osswald, H. (1989). Generation of nitric oxide by human neutrophils. Biochem Biophys Res Commun, 160, 813-9.

Wu, C., Zhang, J., Abu-Soud, H., Ghosh, D.K. \& Stuehr, D.J. (1996). High-level expression of mouse inducible nitric oxide synthase in Escherichia coli requires coexpression with calmodulin. Biochem Biophys Res Commun, 222, 439-44.

Xia, Y., Roman, L.J., Masters, B.S. \& Zweier, J.L. (1998). Inducible nitric-oxide synthase generates superoxide from the reductase domain. $J$ Biol Chem, 273, 22635-9.

Xie, J., Roddy, P., Rife, T.K., Murad, F. \& Young, A.P. (1995). Two closely linked but separable promoters for human neuronal nitric oxide synthase gene transcription. Proc Natl Acad Sci U S A, 92, 1242-6.

Xie, Q. \& Nathan, C. (1994). The high-output nitric oxide pathway: role and regulation. J Leukoc Biol, 56, 576-82.

Xie, Q.W., Cho, H., Kashiwabara, Y., Baum, M., Weidner, J.R., Elliston, K., Mumford, R. \& Nathan, C. (1994). Carboxyl terminus of inducible nitric oxide synthase. Contribution to NADPH binding and enzymatic activity. J Biol Chem, 269, 28500-5.

Xie, Q.W., Cho, H.J., Calaycay, J., Mumford, R.A., Swiderek, K.M., Lee, T.D., Ding, A., Troso, T. \& Nathan, C. (1992). Cloning and characterization of inducible nitric oxide synthase from mouse macrophages. Science, 256, 225-8.

Xie, Q.W., Kashiwabara, Y. \& Nathan, C. (1994). Role of transcription factor NFkappa B/Rel in induction of nitric oxide synthase. J Biol Chem, 269, 4705-8.

Xie, Q.W., Leung, M., Fuortes, M., Sassa, S. \& Nathan, C. (1996). Complementation analysis of mutants of nitric oxide synthase reveals that the active site requires two hemes. Proc Natl Acad Sci U S A, 93, 4891-6. 
Xie, Q.W., Whisnant, R. \& Nathan, C. (1993). Promoter of the mouse gene encoding calcium-independent nitric oxide synthase confers inducibility by interferon gamma and bacterial lipopolysaccharide. J Exp Med, 177, 1779-84.

Xu, L., Eu, J.P., Meissner, G. \& Stamler, J.S. (1998). Activation of the cardiac calcium release channel (ryanodine receptor) by poly-S-nitrosylation. Science, 279, 234-7.

Xu, W., Charles, I.G., Liu, L., Koni, P.A., Moncada, S. \& Emson, P. (1995). Molecular genetic analysis of the duplication of human inducible nitric oxide synthase (NOS2) sequences. Biochem Biophys Res Commun, 212, 466-72.

Xu, W., Charles, I.G., Moncada, S., Gorman, P., Sheer, D., Liu, L. \& Emson, P. (1994). Mapping of the genes encoding human inducible and endothelial nitric oxide synthase (NOS2 and NOS3) to the pericentric region of chromosome 17 and to chromosome 7, respectively. Genomics, 21, 419-22.

Xu, W., Gorman, P., Sheer, D., Bates, G., Kishimoto, J., Lizhi, L. \& Emson, P. (1993). Regional localization of the gene coding for human brain nitric oxide synthase (NOS1) to 12q24.2-- 24.31 by fluorescent in situ hybridization. Cytogenet Cell Genet, 64, 62-3.

Yamada, K. (1998). [Role of nitric oxide in learning and memory processes]. Nippon Yakurigaku Zasshi, 111, 87-96.

Yamada, K., Otabe, S., Inada, C., Takane, N. \& Nonaka, K. (1993). Nitric oxide and nitric oxide synthase mRNA induction in mouse islet cells by interferon-gamma plus tumor necrosis factor-alpha. Biochem Biophys Res Commun, 197, $22-7$.

Young, A.P., Murad, F., Vaessin, H., Xie, J. \& Rife, T.K. (1995). Transcription of the human neuronal nitric oxide synthase gene in the central nervous system is mediated by multiple promoters. Adv Pharmacol, 34, 91-112.

Yuda, M., Hirai, M., Miura, K., Matsumura, H., Ando, K. \& Chinzei, Y. (1996). cDNA cloning, expression and characterization of nitric-oxide synthase from the salivary glands of the blood-sucking insect Rhodnius prolixus. Eur J Biochem, 242, 807-12.

Zeng, G. \& Quon, M.J. (1996). Insulin-stimulated production of nitric oxide is inhibited by wortmannin. Direct measurement in vascular endothelial cells. $J$ Clin Invest, 98, 894-8.

Zeng, J., Heuchel, R., Schaffner, W. \& Kagi, J.H. (1991). Thionein (apometallothionein) can modulate DNA binding and transcription activation by zinc finger containing factor Sp1. FEBS Lett, 279, 310-2.

Zhang, J., Dawson, V.L., Dawson, T.M. \& Snyder, S.H. (1994). Nitric oxide activation of poly(ADP-ribose) synthetase in neurotoxicity. Science, 263, 687-9. 
Zhang, J., Patel, J.M. \& Block, E.R. (1997). Molecular cloning, characterization and expression of a nitric oxide synthase from porcine pulmonary artery endothelial cells. Comp Biochem Physiol B Biochem Mol Biol, 116, 485-91.

Zhang, M. \& Vogel, H.J. (1994). Characterization of the calmodulin-binding domain of rat cerebellar nitric oxide synthase. J Biol Chem, 269, 981-5.

Zhang, M., Yuan, T., Aramini, J.M. \& Vogel, H.J. (1995). Interaction of calmodulin with its binding domain of rat cerebellar nitric oxide synthase. A multinuclear NMR study. J Biol Chem, 270, 20901-7.

Zhang, R., Min, W. \& Sessa, W.C. (1995). Functional analysis of the human endothelial nitric oxide synthase promoter. Sp1 and GATA factors are necessary for basal transcription in endothelial cells. $J$ Biol Chem, 270, 15320-6.

Zhu, L., Gunn, C. \& Beckman, J.S. (1992). Bactericidal activity of peroxynitrite. Arch Biochem Biophys, 298, 452-7.

Ziegler, D.M. (1988). Flavin-containing monooxygenases: catalytic mechanism and substrate specificities. Drug Metab Rev, 19, 1-32.

Zoche, M., Bienert, M., Beyermann, M. \& Koch, K.W. (1996). Distinct molecular recognition of calmodulin-binding sites in the neuronal and macrophage nitric oxide synthases: a surface plasmon resonance study. Biochemistry, 35, 8742-7. 